\title{
WestVirginiaUniversity
}

THE RESEARCH REPOSITORY @ WVU

Graduate Theses, Dissertations, and Problem Reports

2012

\section{Multispectral scleral patterns for ocular biometric recognition}

Simona G. Crihalmeanu

West Virginia University

Follow this and additional works at: https://researchrepository.wvu.edu/etd

\section{Recommended Citation}

Crihalmeanu, Simona G., "Multispectral scleral patterns for ocular biometric recognition" (2012). Graduate Theses, Dissertations, and Problem Reports. 4843.

https://researchrepository.wvu.edu/etd/4843

This Dissertation is protected by copyright and/or related rights. It has been brought to you by the The Research Repository @ WVU with permission from the rights-holder(s). You are free to use this Dissertation in any way that is permitted by the copyright and related rights legislation that applies to your use. For other uses you must obtain permission from the rights-holder(s) directly, unless additional rights are indicated by a Creative Commons license in the record and/ or on the work itself. This Dissertation has been accepted for inclusion in WVU Graduate Theses, Dissertations, and Problem Reports collection by an authorized administrator of The Research Repository @ WVU.

For more information, please contact researchrepository@mail.wvu.edu. 


\title{
MULTISPECTRAL SCLERAL PATTERNS \\ FOR OCULAR BIOMETRIC RECOGNITION
}

\author{
Simona G. Crihalmeanu
}

Dissertation submitted to the

Benjamin M. Statler College of Engineering and Mineral Resources at West Virginia University in partial fulfillment of the requirements

for the degree of

Doctor of Philosophy

in

Electrical Engineering

Arun Ross, Ph.D., Chair

Lawrence Hornak, Ph.D.

Donald Adjeroh, Ph.D.

Xin Li, Ph.D.

Vernon Odom, Ph.D.

Lane Department of Computer Science and Electrical Engineering

Keywords: biometrics;multispectral;sclera;iris;conjunctival vasculature;segmentation;ocular Morgantown, West Virginia 2012 


\begin{abstract}
MULTISPECTRAL SCLERAL PATTERNS

FOR OCULAR BIOMETRIC RECOGNITION
\end{abstract}

\title{
Simona G. Crihalmeanu
}

Biometrics is the science of recognizing people based on their physical or behavioral traits such as face, fingerprints, iris, and voice. Among the various traits studied in the literature, ocular biometrics has gained popularity due to the significant progress made in iris recognition. However, iris recognition is unfavorably influenced by the non-frontal gaze direction of the eye with respect to the acquisition device. In such scenarios, additional parts of the eye, such as the sclera (the white of the eye) may be of significance. In this dissertation, we investigate the use of the sclera texture and the vasculature patterns evident in the sclera as potential biometric cues. Iris patterns are better discerned in the near infrared spectrum (NIR) while vasculature patterns are better discerned in the visible spectrum (RGB). Therefore, multispectral images of the eye, consisting of both NIR and RGB channels, were used in this work in order to ensure that both the iris and the vasculature patterns are successfully imaged.

The contributions of this work include the following. Firstly, a multispectral ocular database was assembled by collecting high-resolution color infrared images of the left and right eyes of 103 subjects using the DuncanTech MS 
3100 multispectral camera. Secondly, a novel segmentation algorithm was designed to localize the spacial extent of the iris, sclera and pupil in the ocular images. The proposed segmentation algorithm is a combination of regionbased and edge-based schemes that exploits the multispectral information. Thirdly, different feature extraction and matching method were used to determine the potential of utilizing the sclera and the accompanying vasculature pattern as biometric cues. The three specific matching methods considered in this work were keypoint-based matching, direct correlation matching, and minutiae matching based on blood vessel bifurcations. Fourthly, the potential of designing a bimodal ocular system that combines the sclera biometric with the iris biometric was explored.

Experiments convey the efficacy of the proposed segmentation algorithm in localizing the sclera and the iris. The use of keypoint-based matching was observed to result in the best recognition performance for the scleral patterns. Finally, the possibility of utilizing the scleral patterns in conjunction with the iris for recognizing ocular images exhibiting non-frontal gaze directions was established. 


\section{ACKNOWLEDGMENTS}

I wish to express my gratitude to my advisor Dr. Arun Ross for his guidance, knowledge and invaluable assistance throughout my research work. He has made available his support whenever I needed it. I owe my deepest grat-

itude to my supervisor, Dr. Lawrence Hornak for his support during the duration of my studies. Special thank you to the members of the supervisory committee Dr. Donald Adjeroh, Dr. Xin Li and Dr. Odom Vernon that contributes to the success of this study.

I would also like to thank my husband Musat, and my children, Irina and Tudor, for their love and support. 


\section{Contents}

Table of Contents $\quad$ v

List of Figures viii

1 Introduction $\quad 1$

1.1 Iris recognition . . . . . . . . . . . . . . . . . . . . 3

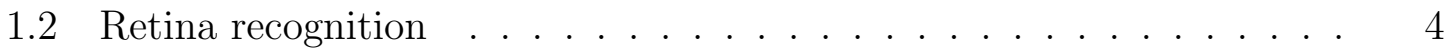

1.3 Periocular region . . . . . . . . . . . . . . . . . . . 6

1.4 Sclera region . . . . . . . . . . . . . . . . 6

1.5 Perceived challenges in scleral patterns processing . . . . . . . . 8

1.6 Summary . . . . . . . . . . . . . . . . . . . . . . . 12

2 Methods for sclera patterns matching using high resolution multi$\begin{array}{ll}\text { spectral images } & 14\end{array}$

2.1 Image acquisition . . . . . . . . . . . . . . . . . . . . 15

2.1.1 From Bayer mosaic pattern to RGB . . . . . . . . . . . . . . 21

2.2 Image denoising . . . . . . . . . . . . . . . . . . . . . 23

2.3 Specular reflection detection and removal . . . . . . . . . . . 25

2.4 Sclera Region Segmentation . . . . . . . . . . . . . . . . 25

2.4.1 Coarse sclera region segmentation: The sclera-eyelid boundary 26

2.4.2 Pupil region segmentation . . . . . . . . . . . . . . 30

2.4.3 Fine sclera region segmentation: The sclera-iris boundary . . . 33

2.5 Enhancement of blood vessels observed on the sclera . . . . . . . . 37

2.6 Image registration . . . . . . . . . . . . . . . . . . . . . . . . . . . . . . . . . . .

2.7 Feature extraction and matching . . . . . . . . . . . . . . . 40

2.7.1 Speeded Up Robust Features (SURF) . . . . . . . . . . . . . . 42

2.7.2 Minutiae detection . . . . . . . . . . . . . . . . . . . . . 43

2.7.3 Direct correlation . . . . . . . . . . . . . . . 46

2.8 Results . . . . . . . . . . . . . . . . . . . 47

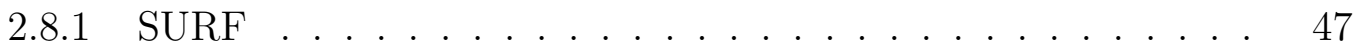

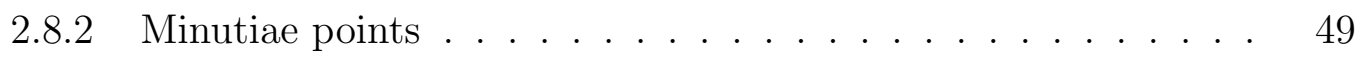

2.8.3 Direct correlation . . . . . . . . . . . . . . . . . . . 49

2.9 Score-level Fusion . . . . . . . . . . . . . . . . . . . . . 50 


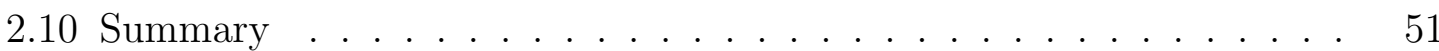

3 Fusion of iris patterns with scleral patterns $\quad \mathbf{5 4}$

3.1 Specular reflection detection and removal . . . . . . . . . . . . 56

3.2 Ocular Region Segmentation . . . . . . . . . . . . . . 57

3.2.1 The Sclera-Eyelid Boundary . . . . . . . . . . . . . . 58

3.2.2 Pupil Region Segmentation ... . . . . . . . . . . 61

3.2.3 The Sclera-Iris Boundary . . . . . . . . . . . . . . . 65

3.2.4 Iris Region Segmentation . . . . . . . . . . . . . . . . . 65

3.2.5 Final Sclera Region Segmentation . . . . . . . . . . . . . 72

3.3 Iris Feature Extraction . . . . . . . . . . . . . . . . 73

3.3.1 Iris Normalization . . . . . . . . . . . . . . . . . . 73

3.3.2 Gabor Wavelets . . . . . . . . . . . . . . . . . . . 75

3.4 Iris Encoding and Matching . . . . . . . . . . . . . . . . 76

3.5 Sclera Feature Extraction and Matching . . . . . . . . . . . . 77

3.6 Results . . . . . . . . . . . . . . . . . . . . . . 77

3.7 Summary . . . . . . . . . . . . . . . . . . . 80

4 Impact of intra-class variation $\quad 81$

4.1 Results . . . . . . . . . . . . . . . . . . 83

4.2 Summary . . . . . . . . . . . . . . . 86

5 Sclera recognition using low resolution visible spectrum images $\quad 88$

5.1 Visible spectrum data set . . . . . . . . . . . . . . 88

5.2 Sclera region segmentation . . . . . . . . . . . . . . . . . . . . . . . . . . . . . . . . 89

5.3 Specular reflection . . . . . . . . . . . . . . . . . 91

5.3.1 General considerations . . . . . . . . . . . . . 91

5.3.2 Detection of specular reflection . . . . . . . . . . . . . 92

5.4 Segmented sclera image without specular reflection . . . . . . . . . . 94

5.5 Image Pre-processing . . . . . . . . . . . . . . . . . . . . . 95

5.6 Matching Results . . . . . . . . . . . . . . . . . . . . . . . 96

5.7 Summary ............................. 97

6 Discussions and Conclusions $\quad 98$

6.1 Future work . . . . . . . . . . . . . . . . 103

A Methods for sclera patterns matching. The ROC and the distribution of scores. Data Collection 1

B Fusion of iris patterns with scleral patterns. The ROC and the distribution of scores. Data Collection 1

C Impact of intra-class variation. The ROC and the distribution of scores. Data collection 2. 
CONTENTS

$\begin{array}{ll}\text { Bibliography } & 141\end{array}$

$\begin{array}{ll}\text { Index } & 147\end{array}$ 


\section{List of Figures}

1.1 Cross-sectional view of the human eye . . . . . . . . . . . . . 2

1.2 View of the frontal iris . . . . . . . . . . . . . . 4

1.3 Illustration of blood vessels in the retina (Adapted from en.wikipedia.org). 5

1.4 Periocular region . . . . . . . . . . . . . . . 6

1.5 Frontal view of the eye . . . . . . . . . . . . . 8

2.1 Block diagram for enhancing and matching multispectral conjunctival vasculature. . . . . . . . . . . . . . . . . . . . . . 15

2.2 The DuncanTech MS3100 camera: CIR/RGB Spectral Configuration (Adapted from Hi-Tech Electronics: www.hitech.com.sg). . . . . . . . 16

2.3 (a) Color-infrared image (NIR-Red-BayerPattern). (b) NIR component. (c) Red component. (d) Bayer pattern. (e) RGB image. (f) Green component. (g) Blue component. (h) Composite image (NIRRed-Green). . . . . . . . . . . . . . . . . . . . .

2.4 (a) Right-eye-looking-left $\left(R_{-} L\right)$. (b) Right-eye-looking-right $\left(R_{-} R\right)$.

(c) Left-eye-looking-left $\left(L_{-} L\right)$. (d) Left-eye-looking-right $\left(L_{-} R\right)$. . . .

2.5 Bayer pattern: a) Bayer pattern grid. b) Green component, red pixel interpolation. c) Green component, blue pixel interpolation (Adapted from www.siliconimaging.com/RGB Bayer.htm). . . . . . . . . . . . .

2.6 Denoising with Double Density Complex Discrete Wavelet Transform. a) Original NIR. b) Denoised NIR. c) Original red component. d) Denoised red component. e) Original green component. f) Denoised green component. g) Original blue component. h) Denoised blue component. Visual differences between original and denoised images are not pronounced due to image rescaling. . . . . . . . . . . . . . .

2.7 (a) Plot of Complex 2-D Double-Density Dual-Tree Wavelets. (b) Iterated filterbank for the Double-Density Complex Discrete Wavelet Transform [1]. . . . . . . . . . . . . . . . . . . . . . . . . . . .

2.8 Specular reflection removal: (a) Original image. (b) Original image with specular reflection removed. . . . . . . . . . . . .

2.9 Sclera region segmentation. The first row displays the original image, the second row displays the normalized sclera index: (a) Dark colored iris. (b) Light colored iris. (c) Mixed colored iris. 
2.10 Sclera region segmentation. The first row displays the results for dark colored iris, the second row displays the results for light colored iris, and the third row displays the results for mixed colored iris: (a) NIR vs.green intensity values. (b) Threshold applied to NSI. (c) Histogram of the NSI. (d) Sclera mask contour imposed on original composite image. 28

2.11 Pupil region segmentation. The first row displays the results for dark colored iris, the second row displays the results for light colored iris: (a) Original image. (b) Convex hull of the sclera region. (c) Hough transform and the highest peak. (d) Sclera region contour and the longest line. (e) The ellipse fitted to the sclera contour. (f) Output of the pupil segmentation algorithm. . . . . . . . . . . . .

2.12 Sclera region segmentation. The first row displays the contour of the sclera cluster and pupil mask, the second row displays the Contour of the convex hull of the sclera cluster and pupil mask imposed on the composite image $I_{S I P}$. (a) Dark colored iris. (b) Light colored iris. (c) Mixed colored iris.

2.13 Sclera region segmentation. The first row displays the results for dark colored iris, the second row displays the results for light colored iris, and the third row displays the results for mixed colored iris: (a) Green component. (b) Red Component. (c) Proportion of sclera in north direction $p_{\uparrow}(x, y)$. (d) Proportion of sclera in south direction $p_{\downarrow}(x, y)$. (e) The proportion of sclera in east direction $p_{\leftarrow}(x, y)$ for left gaze direction. . . . . . . . . . . . . . . .

2.14 Sclera region segmentation. The first row displays the K-means output, the second row displays Contour of the segmented sclera mask imposed on the composite image: (a) Dark colored iris. (b) Light colored iris.(c) Mixed colored iris. . . . . . . . . . . . . . . . . .

2.15 Example of eye images with: (a) Plenty of mascara. (b) Improper illumination. . . . . . . . . . . . . . . . .

2.16 Failure to remove the proper line using Hough transform: (a) Correct detection of the longest line. (b) Incorrect detection of the longest line. 37

2.17 Blood vessel enhancement on the segmented sclera region. (a) Green component of the segmented sclera. (b) Result of the enhancement of blood vessels. (c) The complement image of the enhanced blood vessels 38

2.18 Image registration of the sclera region from images of the same eye. a) Source image. b) Target image. c) Registered source. d) Flow image depicting the warping process. e) Estimated contrast map . . . . . .

2.19 Image registration of the sclera region from two different eyes. a) Source image. b) Target image. c) Registered source. d) Flow image depicting the warping process. e) Estimated contrast map. . . . . 
2.20 The output of the SURF algorithm when applied to enhanced blood vessel images of the same eye (The complement of the enhanced blood vessel images are displayed for better visualization). The number of interest points: 112 and 108. (a) The first 10 pairs of corresponding interest points. (b) All the pairs of corresponding interest points. . .

2.21 The output of the SURF algorithm when applied to enhanced blood vessel images of different eyes (The complement of the enhanced blood vessel images are displayed for better visualization). Number of interest points: 112 and 64. (a) The first 10 pairs of corresponding interest points. (b) All the pairs of corresponding interest points. . . . . . . .

2.22 The centerline of the segmented blood vessels imposed on the green component of two images. . . . . . . . . . . . . . . . .

2.23 Detection of minutiae points. (a) Enhanced blood vessels image. (b) Centerline of the detected blood vessels. (c) Minutiae points: bifurcations (red) and endings (green). . . . . . . . . . . . .

2.24 Failure to detect minutiae points. (a) Enhanced blood vessels image. (b) The detected vasculature without ramifications and intersections (Morphological operations such as dilation is applied to the blood vessels for a better visualization). . . . . . . . . . . . . .

2.25 The histogram (25 bins) of the detected number of interest points for images of the eye (data collection 1). (a) Left-eye-looking-left (L_L). (b) Left-eye-looking-right (L_R). (c) Right-eye-looking-right (R_L). (d) Right-eye-looking-right (R_R) . . . . . . . . . . . . . . . . 48

3.1 Block Diagram . . . . . . . . . . . . . . . . . .

3.2 The sclera-eyelid boundary. The first row displays the results for dark colored iris, the second row displays the results for light colored iris, and the third row displays the results for mix colored iris: (a) original composite image. (b) The normalized sclera index (NSI). (c) The output of the K-means clustering algorithm. (d) Sclera-eyelid boundary imposed on original composite image. . . . . . . . . . . . . . .

3.3 The sclera-eyelid boundary errors. First row represents the errors in images with strong uneven illumination. Second row represents the errors in images with large specular reflections on the skin. (a) Original composite image. (b) Normalized sclera index. (c) The output of the k-means algorithm. . . . . . . . . . . . . . .

3.4 Pupil region segmentation. Filling the holes at the iris-pupil boundary due to inpainting of the specular reflection that results in higher pixel value than the pupil pixel value. . . . . . . . . . . . . . 
3.5 Pupil region segmentation. (a) The metric $M$ for the thresholds 0.04, 0.1, and 0.16. (b) Thresholding result (the contour) imposed on the composite image, thresholds $0.04,0.1$, and 0.16 . (c) The metric $M$ for the thresholds $0.18,0.2$, and 0.24 . (d) Thresholding result (the contour) imposed on the composite image, for thresholds $0.18,0.2$,

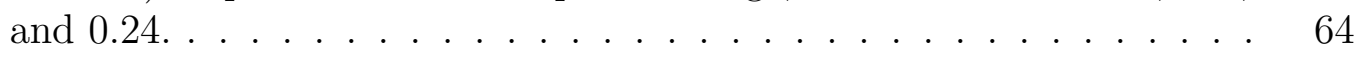

3.6 Pupil region segmentation. Examples. . . . . . . . . . . . . . . . 64

3.7 Ocular images with a greater amount of melanin around the iris region. 65

3.8 Iris segmentation. Elliptical unwrapping based on the pupil parameters: (a)Dark colored iris . (b) Light colored iris . (c) Mixed colored iris. . . . . . . . . . . . . . . . . . .

3.9 Iris segmentation. The first row displays the results for dark colored iris, the second row displays the results for light colored iris, and the third row displays the results for mix colored iris: (a) Pupil mask unwrapped. (b) Sclera mask unwrapped. . . . . . . . . . . . . . . . . . .

3.10 Iris segmentation. Contour of the two ellipses, Ellipse min $_{\text {and }}$ Ellipse max $_{\text {, }}$, and their tilt imposed on the composite image. . . . . . . . . . . . . . 70

3.11 Iris segmentation: (a) Color gradient on composite image. (b) The

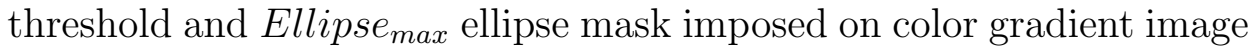
ColorGradth. . . . . . . . . . . . . . . . . . . 70

3.12 Iris segmentation. Image Color $\operatorname{Grad}_{T h}$ unwrapped along with the two

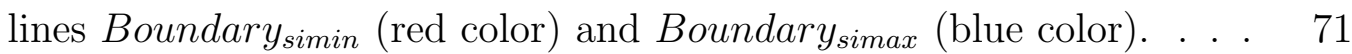

3.13 Examples of segmented irides: (a) Dark colored iris. (b) Light colored iris. (c) Mixed colored iris. . . . . . . . . . . . . . . . . . . 71

3.14 Examples of correct eye image segmentation. . . . . . . . . . . . . . . 72

3.15 Examples of correct eye image segmentation. . . . . . . . . . . . . . . 72

3.16 Iris normalization: Daugman rubber sheet model. . . . . . . . . . . . 74

3.17 Examples for iris normalization: (a) Normalized iris. (b) The mask for occlusions. . . . . . . . . . . . . . . . 74

3.18 Gabor wavelet . . . . . . . . . . . . . . . . 75

3.19 Phase quantization: (a) Four levels represented by the sign of the $\mathrm{Im}$ and $R e$ for every quadrant (0 - negative, 1 - positive). (b) Example of iris template. . . . . . . . . . . . . . . . . 76

4.1 Block Diagram . . . . . . . . . . . . . . . . . . . . . 82

5.1 Near images of the eye where the subject is: a) looking straight ahead, b) looking up, c) looking left, d) looking right. . . . . . . . . . .

5.2 Segmenting the sclera from two different eye images, displayed by column: a) Original image, b) Segmented sclera region based on RGB values (red $=$ sclera region, blue $=$ iris region, black $=$ the background) c) Convex hull of the sclera (blue+red) containing a portion of the iris (blue) 
5.3 Segmenting the sclera of two different eye images: a) Original image, b) Sclera mask, c) Segmented sclera region. . . . . . . . . . . . . 99 90

5.4 Plots of the equation 5.1 for various values of $\gamma ; \mathrm{c}=1$ in all cases . . 92

5.5 Detection of specularities. Examples for $\gamma=3$ : (a) Illumination component of HSI sclera image; (b) Histogram of the illumination component; (c) Filtered envelop of the histogram . . . . . . . . . . . . . 94

5.6 Example of threshold values for different values of $\gamma \ldots \ldots$. . . . 94

5.7 Detecting specularities: a) Original image, b) Threshold values for $1 \leq \gamma \leq 10$ c) Specular reflection mask . . . . . . . . . . . .

5.8 Segmenting the sclera after removing specularities: a) Original image, b) Specular reflection mask c) Segmented sclera without specular reflection . . . . . . . . . . . . . . . . . . . . .

5.9 Image Enhancement:(a) Original sclera vein image, (b) Enhanced sclera vein image. . . . . . . . . . . . . . . . . . . . . . . 99 96

5.10 Example of pre-processed sclera vein image. . . . . . . . . . . 96

5.11 ROC curve indicating the results of matching $\ldots \ldots \ldots \ldots$

A.1 Data collection 1. The ROC and the distribution of scores for the SURF technique. . . . . . . . . . . . . 106

A.2 Data collection 1. The ROC and the distribution of scores for the minutiae-based matching technique. . . . . . . . . . . . 107

A.3 Data collection 1. The ROC and the distribution of scores for the correlation technique. . . . . . . . . . . . . . . . 108

A.4 Data collection 1. The ROC and the distribution of scores for the mutual information technique. . . . . . . . . . . . . . . 109

A.5 Data collection 1. The ROC and the distribution of scores for the normalized mutual information technique. . . . . . . . . . 110

A.6 Data collection 1. The ROC and the distribution of scores for the ratio-image uniformity technique. . . . . . . . . . . . . . 111

A.7 Data collection 1. The ROC and the distribution of scores for the root mean square error technique. . . . . . . . . . . . . . . . . 112

A.8 Data collection 1. The ROC and the distribution of scores for the structural similarity index technique. . . . . . . . . . . . . . 113

A.9 Data collection 1. The ROC and the distribution of scores for the fusion of minutiae and correlation technique. . . . . . . . . . . 114

A.10 Data collection 1. The ROC and the distribution of scores for the fusion of minutiae and mutual information technique. . . . . . . . 115

A.11 Data collection 1. The ROC and the distribution of scores for the fusion of minutiae and normalized mutual information technique. . .

A.12 Data collection 1. The ROC and the distribution of scores for the fusion of minutiae and ratio-image uniformity technique. . . . . . . 117

A.13 Data collection 1. The ROC and the distribution of scores for the fusion of minutiae and root mean square error technique. . . . . . . 118 
A.14 Data collection 1. The ROC and the distribution of scores for the fusion of minutiae and structural similarity index technique. . . . . .

B.1 Data collection 1. The ROC and the distribution of scores for the SURF technique (automatic sclera segmentation). . . . . . . .

B.2 Data collection 1. The ROC and the distribution of scores for the Hamming distance. . . . . . . . . . . . . . . . . .

B.3 Data collection 1. Fusion of iris patterns and sclera patterns. The ROC and the distribution of scores for $L_{\_} \mathrm{L}$, simple sum rule, maximum rule, minimum rule. . . . . . . . . . . . . . . . . . . .

B.4 Data collection 1. Fusion of iris patterns and sclera patterns. The ROC and the distribution of scores for $L_{-} \mathrm{R}$, simple sum rule, maximum rule, minimum rule. . . . . . . . . . . . . . . . . . . . . . . .

B.5 Data collection 1. Fusion of iris patterns and sclera patterns. The ROC and the distribution of scores for R_L, simple sum rule, maximum rule, minimum rule. . . . . . . . . . . . . . . . . . . .

B.6 Data collection 1. Fusion of iris patterns and sclera patterns. The ROC and the distribution of scores for $R \_R$, simple sum rule, maximum rule, minimum rule. . . . . . . . . . . . . . . 126

C.1 Data collection 2. The ROC and the distribution of scores for the SURF technique. . . . . . . . . . . . . . . . . .

C.2 Data collection 2. The ROC and the distribution of scores for the minutiae-based matching technique. . . . . . . . . . . .

C.3 Data collection 2. The ROC and the distribution of scores for the correlation technique. . . . . . . . . . . . .

C.4 Data collection 2. The ROC and the distribution of scores for the mutual information technique. . . . . . . . . . . . . . . . 131

C.5 Data collection 2. The ROC and the distribution of scores for the normalized mutual information technique. . . . . . . . . . . .

C.6 Data collection 2. The ROC and the distribution of scores for the ratio-image uniformity technique. . . . . . . . . . . . .

C.7 Data collection 2. The ROC and the distribution of scores for the root mean square error technique. . . . . . . . . . . . . . . . . 134

C.8 Data collection 2. The ROC and the distribution of scores for the structural similarity index technique. . . . . . . . . . .

C.9 Data collection 2. The ROC and the distribution of scores for the Hamming distance. . . . . . . . . . . . . . . 136

C.10 Data collection 2. The fusion of iris and sclera patterns for left-eyelooking-left. The ROC and the distribution of scores. . . . . . . . .

C.11 Data collection 2. The fusion of iris and sclera patterns for left-eyelooking-right. The ROC and the distribution of scores. . . . . . . . . 138 
C.12 Data collection 2. The fusion of iris and sclera patterns for right-eyelooking-left. The ROC and the distribution of scores. . . . . . . . . 139

C.13 Data collection 2. The fusion of iris and sclera patterns for right-eyelooking-right. The ROC and the distribution of scores. . . . . . . . 140 


\section{List of Tables}

2.1 Specifications for DuncanTech MS3100 _ . . . . . . . . . . 17

2.2 Performance specifications for DuncanTech MS3100 . . . . . . . 18

2.3 High resolution multispectral database . . . . . . . . . . . . . . 21

2.4 The EER (\%) results when using SURF. . . . . . . . . . . . . . 49

2.5 The average number of detected interest points for data collection 1.49

2.6 The EER (\%) results when using minutiae points. . . . . . . . . . 49

2.7 The EER (\%) results when using different correlation methods. . . . 50

2.8 The EER (\%) results of the fusion of minutiae scores with different correlation measures. . . . . . . . . . . . . . . . . . 52

3.1 The EER (\%) results for iris patterns using Hamming distance. . . . 78

3.2 The EER (\%) results for scleral patterns when using SURF. . . . . 78

3.3 The EER (\%) results of the fusion of iris patterns (Hamming distance) and scleral patterns $(\mathrm{SURF}) \ldots \ldots \ldots \ldots . \ldots \ldots$

3.4 Average computation times . . . . . . . . . . . . . . . . . . . 79

4.1 The EER (\%) results for scleral patterns when using SURF. . . . . . 84

4.2 The EER (\%) results for scleral patterns when using minutiae points. 84

4.3 The EER (\%) results for scleral patterns when using different correlation methods. . . . . . . . . . . . . . . . . 85

4.4 The EER (\%) results for iris patterns using Hamming distance. . . . 86

4.5 The EER (\%) results of the fusion of iris patterns (Hamming distance) and scleral patterns $(\mathrm{SURF}) \ldots \ldots \ldots \ldots$

5.1 Manual correction statistics of segmented sclera $\ldots \ldots \ldots \ldots$ 


\section{Chapter 1}

\section{Introduction}

Biometrics is the science of recognizing a person based on physical traits such as face, fingerprint, and iris or behavioral characteristics such as gait, keystroke dynamics, and signature [2]. Conventional techniques to authenticate an individual are based on identification cards (something that you carry) and passwords or PINs (something that you know). An issue associated with these ways of authentication is that the cards, passwords and PINs can be stolen, lost, or simply forgotten. The need to reliably determine and verify the identity of a person in a convenient, easy, and accessible way has stimulated intense research in the field of biometric authentication.

Rooted in the latin word oculus that means eye, the term ocular biometrics is used to bring together all the biometric modalities associated with the eye and its surrounding region. Defined as an organ of sight, a specialized light-sensitive sensory structure, the eye allow us to observe and learn about the surrounding world. A cross section (Figure 1.1) reveals the main parts of the eye: the extraocular muscles that controls the movement of the eyeball within the eye socket; the three layers surrounding the eyeball, the sclera, the choroid and the retina with the cornea, the iris and conjunctiva in front; the anterior and posterior chambers separated by the 


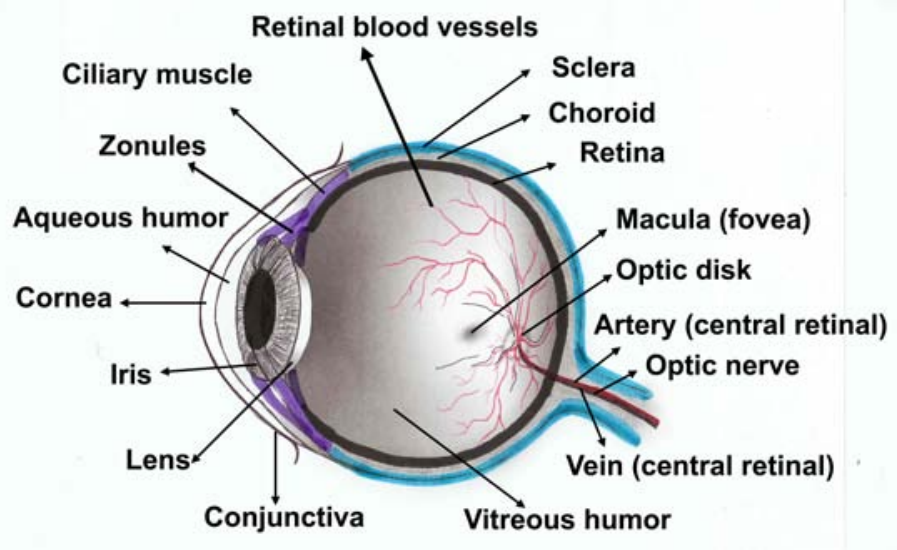

Figure 1.1 Cross-sectional view of the human eye

lens and filled with aqueous humor and vitreous humor; the optic nerve and the macula with the fovea.

In the course of time, parts of the eye were proved to be powerful discriminatory features among individuals. The most famous are the rich texture of the iris (Section 1.1) and the blood vessels observed on the retina (Section 1.2). Recent studies are considering the shape of the eye and its surrounding region, named periocular region, as a possible biometric cue (Section 1.3). The present work attempts to prove that the sclera texture and the vasculature patterns exhibited on its surface, Figure 1.5 may be considered an ocular biometric. Like any other biometric modality, the sclera texture and the vasculature patterns have to conform with the well known criteria [3]: universality, uniqueness, permanence, acceptability, collectivity and circumvention. The sclera is a part of the natural anatomy of the human eye, rendering its universality. As a relatively new research topic in the field of biometrics, no previous thorough analysis has been conducted in regards to its uniqueness. However, studies on other vascular tissues, such as the fundus of the eye, confirms the distinctiveness of blood 
vessel patterns, has provided evidence of the long lasting form of the vessels and their durability through the years as age progresses. Outside factors such as exposure to chemicals, physical trauma, and some diseases may influence the appearance of the sclera region. Images of the sclera region are easily collected with commercial digital cameras when a proper amount of light is directed towards the eye, and therefore it can be easily photographed without intrusion and discomfort to the subject. Staring at a specific point is not required, and only a fraction of a second is needed to capture the image of the eye; the subject is not obligated to be stationary for long periods of time. Reproducing the rich texture of the sclera region is difficult, making this modality invulnerable to imitation and tampering.

\subsection{Iris recognition}

Among various biometric modalities, the iris has been assumed to be reliable, accurate, and stable over long periods of time [4], [5], [6], [7], [8]. The iris is the annular colored structure in the eye located behind the cornea and the aqueous humour, and in front of the lens (Figure 1.2). Its role is to control the amount of light that enters the eye through the central opening called the pupil. The iris has two sets of muscles: a sphincter muscle around the pupil that contracts in bright lighting, thereby decreasing the pupil size, and one that radiates outward from the pupil, enlarging it in dim light. The frontal view of the iris is separated into two regions called the pupillary zone and the ciliary zone. They are separated by the collarette, which is the thickest region in the iris and where the sphincter and the dilater muscles overlap. Visible features of the iris that are important for recognition are the collarette; the pigment

related features, such as crypts and pigment spots [9]; the features controlling the size of the pupil, such as radial and concentric furrows; and the visible anomalies due 


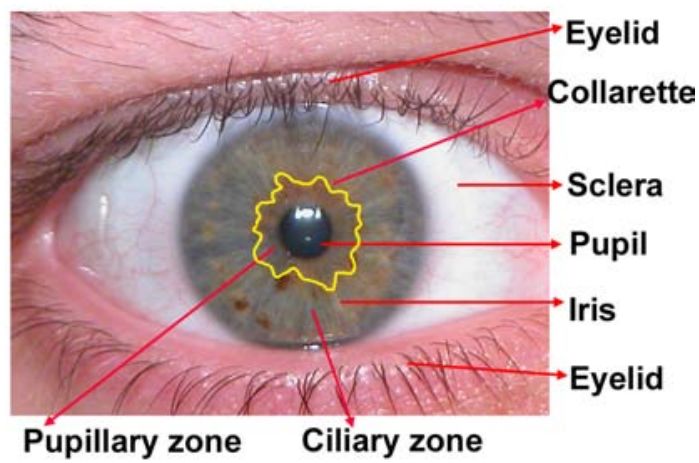

Figure 1.2 View of the frontal iris

to aging and trauma [10]. The advantages of using the iris for recognition are that it is protected by the cornea, though it is still visible; it is acquirable from a distance and presents good processing speed; it has a very rich texture, therefore affording a high degree of randomness and uniqueness; it is difficult to tamper surgically. More over, there are two irides, that are different even for twins. However, the iris is obstructed by eyelashes, and specific lighting can cause specular reflection due to the iris's location behind a curved, wet surface. Also, the iris contracts and dilates constantly, causing variable non-linear deformations. The matching performance of the iris recognition system is greatly and unfavorably influenced by the direction of the gaze of the eye and the distance with respect to the acquisition device.

\subsection{Retina recognition}

A more dated identification method based on vasculature patterns are retinal scans [3], [11], [12]. The retina, the innermost layer of the eye, is a sensory tissue that acts as a camera film to create an image. It contains millions of photo-receptors called cones and rods, which capture the incoming light, and through chemical and electrical processes, trigger electrical impulses. These impulses advance along the optical nerve 


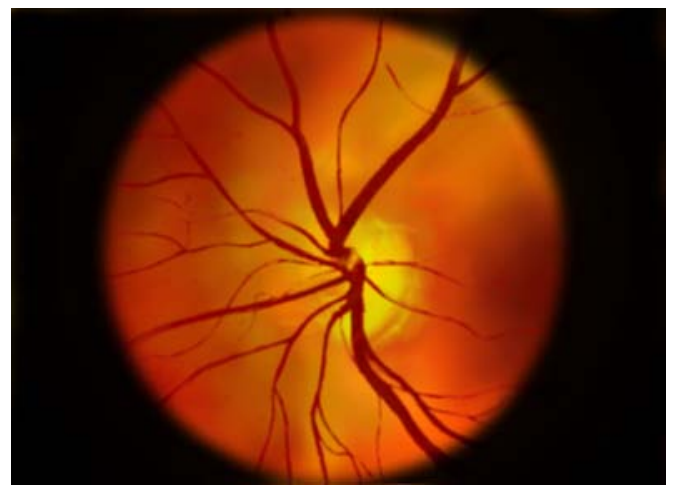

Figure 1.3 Illustration of blood vessels in the retina (Adapted from en.wikipedia.org).

to the brain where image is formed. The light is focused on the retina in the macula. The center of the macula is called the fovea which is accountable for sharp vision. The cones are used for detecting bright light and colors, mostly packed in the fovea. The rods are used for dim light and peripheral and night vision. They dominate the peripheral macula. The retina exhibits a myriad of blood vessels, all converging towards the optical disk. Their richness and tortuosity are highly accurate, difficult to forge and stable in time (Figure 1.3). Since retina decays rapidly after death the detection of vitality is not necessary. However, capturing the image of the back of the eye is invasive, slow, and expensive. A beam of infrared light is directed into the pupil for 10 to 15 seconds to illuminate the retina. This creates discomfort for the subject and is perceived as a health threat by the general public. Medical conditions that affect retinal scans are eye diseases such as cataract and glaucoma. Suited for environments requiring maximum security - in the military and government - retinal scans are unlikely to be deployed for general public use. 


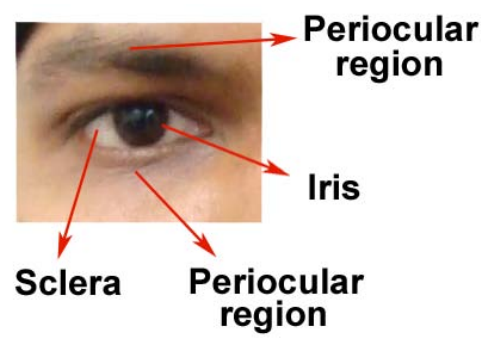

Figure 1.4 Periocular region

\subsection{Periocular region}

Recently, researchers explored the feasibility of using the periocular region, defined as the region surrounding the eye, Figure 1.4, as a biometric cue [13], [14], [15]. The periocular region's role is significant when the eye is occluded (e.g., due to blinking). Its advantages are that it can be captured without strict viewing angle and distance constraints, and it does not necessarily require the subject's cooperation. Eye shape and skin texture encoded by local or global features can be used as soft biometrics [14]. A limitation of the periocular region is that the features are not as distinguishable as iris, resulting in lower accuracy.

\subsection{Sclera region}

In contrast, visible on the sclera (the white of the eye), a structure of fine blood vessels may be imaged without discomfort for the subject (Figure 1.5). These patterns, composed of more or less prominent veins, are visible at all times, except when the gaze direction is downward and the eye is covered by the eyelid. Their richness is increased in non-frontal eye images, thus allowing the sclera region to be used in addition to iris recognition for more complete and accurate identification. The sclera [16], [17] is the external layer of the eye, a protective coat serving to maintain 
the form of the eyeball under the pressure of the eye's internal liquids. It also provides an attachment for ligaments and the six extraocular muscles which rotate the eyeball in the eye socket. It is a firm, dense membrane with thickness that varies from $0.8 \mathrm{~mm}$ to $0.3 \mathrm{~mm}$, containing collagen and elastic fibers. The sclera is organized in four layers (from outermost to innermost): episclera, stroma, lamina fusca, and endothelium. The sclera is generally avascular; only its outer surface, the episclera, contains the blood vessels that nourish it. In the back of the eye, the sclera is continued with the dural sheath of the optic nerve, and anterior sclera is completed by the cornea (a clear and transparent layer at the center of the eye, located in front of the iris, and whose main purpose is to focus the light as it enters the eye). The anterior part of the sclera, up to the edge of the cornea (the sclero-corneal junction), and the inside of the eyelid, are covered by the conjunctival membrane. The conjunctival membrane is a thin layer containing secretory epithelium that helps lubricate the eye for eyelid closure and protects the ocular surface from bacterial and viral infections. The part of the conjunctiva that covers the inner lining of the eyelids is called palpebral conjunctiva. The part of the conjunctiva that covers the outer surface of the eyeball is called bulbar conjunctiva and the area where palpebral conjunctiva becomes the bulbar conjunctiva is called the conjunctival fornix. The conjunctiva is semitransparent, colorless, and contains blood vessels. Anatomically, the blood vessels in bulbar conjunctiva can be differentiated from those of the episclera. While the conjunctival blood vessels can slightly move with the conjunctival membrane, those in episclera will not [18]. The rich vasculature patterns revealed in the episclera and conjunctival membrane are together referred to as conjunctival vasculature in this study. 


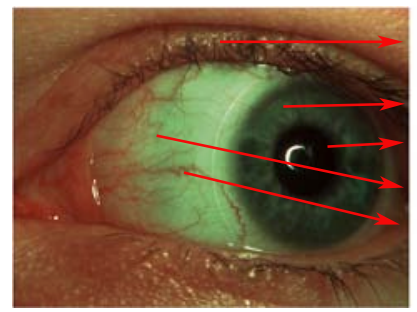

\section{Eyelid}

Iris

Pupil

Sclera region

Blood vessels in the sclera

Figure 1.5 Frontal view of the eye

\subsection{Perceived challenges in scleral patterns pro- cessing}

Acquiring and processing images of the sclera surface and the conjunctival vasculature patterns is challenging due to the approximately spherical surface of the eyeball, external lighting conditions, anatomical structure of the layers of the sclera and the fineness of vascular patterns. In order to image the scleral surface with its blood vessel patterns, a sufficient amount of light has to be directed into the eye. This light touches the spherical surface of the sclera, after passing through conjunctiva. Optical processes such as reflection and refraction are difficult to control and create specular reflections with a large variety of intensity values, different topologies, shapes, sizes and locations. The randomness of the specular reflection is accentuated by the rapid movement of the eyeball. According to the angle of incoming ray of light to the sclera, some specular highlights have intensities close to the background intensity values; hence detecting them is difficult. Because of the round shape of the eye, there are large variations in intensity values across the sclera surface. Further, due to the low contrast between the blood vessels in the foreground and the texture in the background, classical methods of segmentation based on edge detection are less robust and do not provide good results. Therefore, alternative methods that

overcome the problem of intensity variations have to be used. Upon rubbing the 
eye, the conjunctiva can slightly move relative to the sclera surface generating small deformations of its blood vessels. Matching the conjunctival vasculature is greatly impacted by these deformations. Medical conditions can also influence the performance of the segmentation and feature extraction algorithms in all processing steps. Diabetes, for example, is associated with the loss of capillaries, macrovessel dilation and changes in tortuosity in conjunctiva [19], [20]. Hyperemia, conjunctivitis, hemorrhage, episcleritis [18] influence the amount of blood vessels seen on the sclera surface and affect the contrast between the blood vessels and the background (the eye becomes reddish). Age is another factor that influences the appearance of the sclera surface. Conjunctiva can stretch and loosen from the underlying sclera leading to the formation of conjunctival folds. The white of the eye becomes yellowish and an increase in melanin in conjunctiva can also appear. In younger people, blood vessels may be less visible. With age the conjunctival vasculature becomes thicker and more visible.

The implicit image challenges enumerated above makes sclera segmentation process a difficult task. An accurate segmentation of the sclera region along the eyelid contour and the iris boundary is important to further prevent challenges at feature extraction level. When the entire image of the eye is used for processing, the blood vessels are difficult to distinguish among other different types of lines such as wrinkles, crows feet, and eyelashes. Therefore, a good segmentation of the sclera region that clearly exhibits the blood vessels is necessary. The sclera is not a uniform white; more over approaching the lacrimal caruncle (caruncula lachrymalis ${ }^{1}$, a small, pink, globelike nodule located at the corner of the eye), the sclera gradually adopts a reddish tint. Due to the natural redness of the sclera near the corner of the eye, the pixels pertaining to the sclera region are difficult to differentiate from the pixels pertaining

\footnotetext{
${ }^{1}$ en.wikipedia.org
} 
to the skin. Also, an improper illumination of the curved surface of the eye, may result in darker regions in the corner of the eye opposite to lacrimal caruncle. These issues create problems for the segmentation process.

The thresholding and clustering methods (k-means) for segmentation are employed in the presented work with some degree of performance. The key in thresholding method is to select the proper threshold [21]. A common threshold that is optimal for the segmentation of the sclera in all the images is difficult to find. When the clustering method k-means is used, the final solution depends largely on the initial set of clusters. The algorithm is fast but does not guarantee a good sclera-eyelid contour detection.

The histogram-based segmentation method [22] consists of finding the peaks and valleys in the chart in order to locate clusters. The image of the eye is divided in four regions of interest: the iris; the sclera; the skin and the pupil, eyelashes included. This method is fast; it takes one pass to read the intensity value of the pixels and to create the histogram. However, it is difficult to identify which peaks (clusters) and valleys (the boundaries between the regions of interest) are significant. The four clusters often overlap, meaning they are undistinguishable.

Boundaries between regions that are to be segmented are characterized by changes in the intensity value of pixels. They are known as edges. There are many different ways in which edges can be detected [23], [21], [22]. Unfortunately, these methods result in a multitude of disconnected segments from which it is difficult to select the proper boundaries of the sclera region. It is also challenging and problematic to continue the gaps along the contour between the sclera and the eyelids.

Region growing, and split-and merge methods [21] were employed without satisfactory results due to the large variation is intensity values across the sclera region. There are other algorithms that could potentially be used to segment the sclera region 
such as partial differential equations (level set), graph partitioning and many others.

The image of the iris is better discerned in near infrared (NIR) spectrum, while vasculature patterns are better observed in the visible spectrum (RGB). Therefore, multispectral images of the eye (Section 2.1), consisting of both NIR and RGB channels, were used in this work in order to ensure that both the iris and the vasculature patterns are successfully imaged. This dissertation is the first extensive work and first attempt to demonstrate the validity of using the sclera texture and the conjunctival vasculature visible on its surface as a new biometric modality. This idea came to reality with the submission of Dr. R. Derakhshani's and Dr. Arun Ross' patent [24]. This work include the following enumerated contributions. Firstly, a multispectral ocular database (2 collections of 103 and 31 subjects) was assembled by collecting high-resolution color infrared images of the left and right eye using the DuncanTech MS3100 multispectral camera under constrained conditions (Chapter 2). Secondly, a novel segmentation algorithm was designed to localize the spatial extent of the iris, sclera and pupil in the ocular images. The proposed segmentation algorithm is a combination of region-based and edge-based schemes that exploits the multispectral information (Chapter 2, 3). Thirdly, different feature extraction and matching methods were used to determine the potential of utilizing the sclera and the accompanying vasculature pattern as biometric cues. The three specific matching methods considered in this work were keypoint-based matching, direct correlation matching, and minutiae based on blood vessel bifurcations (Chapter 2, 4). Fourthly, the potential of designing a bimodal ocular system that combines the sclera biometric with the iris biometric was explored (Chapter 3, 4). It is well demonstrated that iris recognition performance is greatly and negatively influenced by the occlusions, the lighting conditions and by the direction of the gaze of the eye with respect to the acquisition device [25]. The more the gaze direction deviates from the frontal pose, 
the more information from the iris texture is lost and the more information from the sclera region is gained. Depending on the richness and locality of the conjunctival vasculature exposed on the surface of the sclera, prominent veins may be visible when iris is occluded. More over, the combined sclera and the iris texture may be used in non-cooperative recognition. In the attempt to establish the utility of the sclera region as a biometric cue, the analysis of the sclera surface is also considered in low resolution, visible spectrum, unconstrained images. The purpose is to evaluate the matching performance of the sclera texture and the blood vessels in each case and to compare their performances when moving from high to low resolution images, and changing the lighting conditions, acquiring distances and viewing angles (Chapter 5).

\subsection{Summary}

This chapter is an introduction to biometrics as a discipline. It presents the ocular biometric modalities, each with its advantages and disadvantages. Among these modalities, iris recognition gained popularity in the last decade due to its reliability, stability for long period of times and accuracy. However, the matching performance of the iris recognition system is greatly and unfavorably influenced by the direction of the gaze of the eye with respect to the acquisition device. To compensate the loss of information in non-frontal images, the sclera texture and the blood vessels seen on its surface are proposed as a new biometric modality to be combined with the iris patterns. An anatomical introduction of the sclera is presented along with the perceived challenges in scleral pattern processing. General segmentation methods used with some degree of success to localize the sclera region are presented. The chapter ends by enumerating the contributions of this work to initiate research in this field, mainly to investigate the use of sclera texture and the conjunctival vasculature as a 
biometric cue and the potential of designing a bimodal ocular system that combines the scleral patterns with the iris patterns is proposed. 


\section{Chapter 2}

\section{Methods for sclera patterns}

\section{matching using high resolution multispectral images}

In order to prove the feasibility of using the sclera surface in an ocular biometric

system, it is essential to determine what features could be exploited for identification, how powerful is their discriminatory potential, and what methods are the most effective to characterize this biometric. In this dissertation, the design of three different feature extraction and matching methods is presented. The first one is based on interest-point detection and utilizes the entire sclera region including the vasculature pattern; the second is based on minutiae points on the vasculature structure; and the third is based on direct correlation. The block diagram of the proposed system is shown in Figure 2.1. In this approach the multispectral collection 1 is used. 
Chapter 2 Methods for sclera patterns matching using high resolution multispectral images

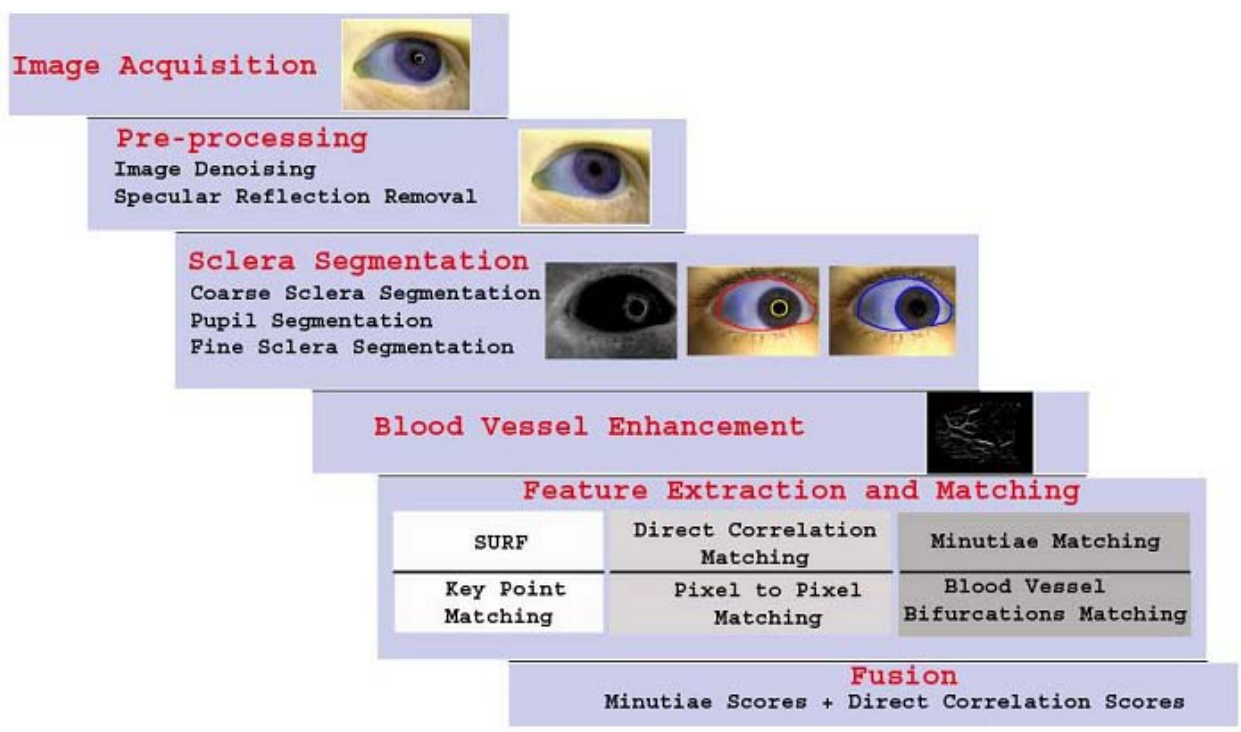

Figure 2.1 Block diagram for enhancing and matching multispectral conjunctival vasculature.

\subsection{Image acquisition}

Multispectral imaging captures the image of an object at multiple spectral bands often ranging from the visible spectrum to the infra-red spectrum. The small visible spectrum band [21] is represented by three narrow sub-bands called the red, green and blue channels, that range from $0.4 \mu \mathrm{m}$ to $0.7 \mu \mathrm{m}$. The infrared spectrum is divided into NIR (near infrared), MIR (midwave infrared), FIR (far infrared) and thermal bands, ranging from $0.7 \mu \mathrm{m}$ to over $10 \mu \mathrm{m}$. In this work, images of the eye were collected using the Redlake (DuncanTech) MS3100 multispectral camera ${ }^{1}$. The camera has three array sensors based on CCD technology. Between the lenses and the sensors there is a color-separating prism to split the ingoing broadband light into three optical channels. The camera acquires imagery of four spectral bands from a three channel optical system (Figure 2.2). The CIR (color Infrared)/RGB configuration outputs

\footnotetext{
${ }^{1}$ Hi-Tech Electronics, Spectral Configuration Guide for DuncanTech 3-CCD Cameras, http://www.hitech.com.sg
} 
Chapter 2 Methods for sclera patterns matching using high resolution multispectral images

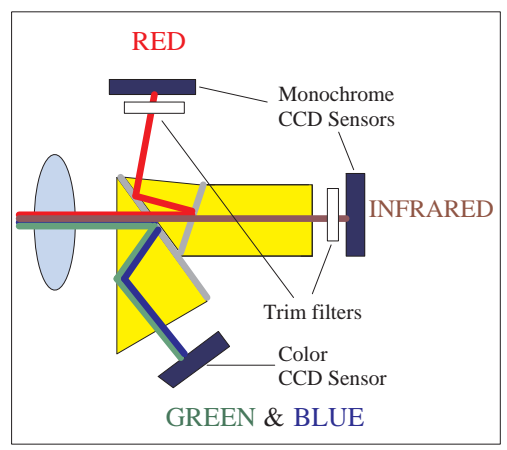

Figure 2.2 The DuncanTech MS3100 camera: CIR/RGB Spectral Configuration (Adapted from Hi-Tech Electronics: www.hitech.com.sg).

three channels represented as a 2D matrix of pixels that are stacked on top of each other along the third dimension; the three channels correspond to the near-infrared (NIR), red component, and a Bayer mosaic-like pattern [26].

Some of the characteristics of the multispectral camera, as described in the datasheet ${ }^{2}$, are displayed in the Table 2.1 and 2.2 .

Figure 2.3 shows an example of a CIR image along with its components. The first channel - the NIR component - is stored as a separate image. The second channel the red component - is stored as the red component of the RGB image. The green and blue components are obtained from the third channel of the CIR/RGB configuration through a Bayer pattern demosaicing algorithm ${ }^{3}$ described in Section 2.1.1. The red pixels on the Bayer color array are ignored. As specified by the sensor manufacturer, the center wavelength of each spectral band is as follows: blue - $460 \mathrm{~nm}$, green- 540 $\mathrm{nm}$, red - $660 \mathrm{~nm}$, and NIR - $800 \mathrm{~nm}$.

The interface used to collect multispectral images is composed of an ophthalmologist's slit-lamp mount and a light source. The mount consists of a rest chin to position the head and a mobile arm to which the multispectral camera is attached.

\footnotetext{
${ }^{2}$ www.GeospatialSystems.com

${ }^{3}$ RGB "Bayer" Color and MicroLenses, www.siliconimaging.com/RGB Bayer.htm
} 
Chapter 2 Methods for sclera patterns matching using high resolution multispectral images

Table 2.1 Specifications for DuncanTech MS3100

\begin{tabular}{||l||}
\hline \hline Snapshot \\
\hline \hline Color separating prism with 3 CCD imaging sensors \\
\hline $1392(\mathrm{H}) \times 1040(\mathrm{~V})$ resolution $(\mathrm{x} 3), 4.3$ million pixels of data \\
\hline Image $3-5$ spectral bands from $400-1100 \mathrm{~nm}$ \\
\hline Standard models for RGB, CIR, and RGB/CIR \\
\hline Custom multispectral configurations \\
\hline Frame rates up to 7.5 fps \\
\hline "Smart Camera" features for advanced control and processing \\
\hline Display composite, false color, or individual color plane images \\
\hline Independent gain, offset, and exposure control for each channel \\
\hline RS-232 input for configuration and control \\
\hline \hline
\end{tabular}

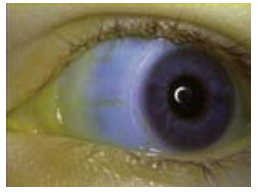

(a)

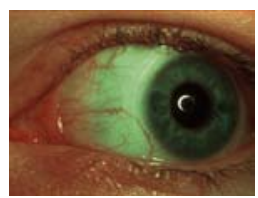

(e)

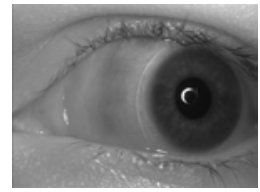

(b)

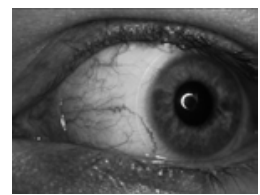

(f)

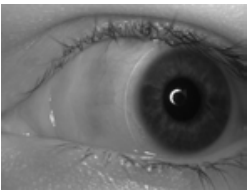

(c)

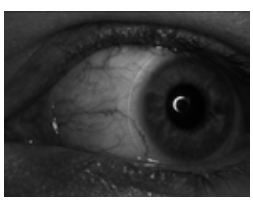

(g)

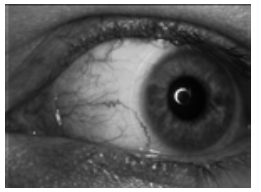

(d)

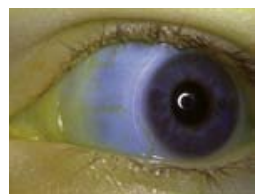

(h)

Figure 2.3 (a) Color-infrared image (NIR-Red-BayerPattern). (b) NIR component. (c) Red component. (d) Bayer pattern. (e) RGB image. (f) Green component. (g) Blue component. (h) Composite image (NIR-Red-Green). 
Chapter 2 Methods for sclera patterns matching using high resolution multispectral images

Table 2.2 Performance specifications for DuncanTech MS3100

\begin{tabular}{|c|c|}
\hline Performance specifications & \\
\hline Image device & 3 of $1 / 2$ inch Interline Transfer CCD \\
\hline Pixel size & $4.65 \times 4.65 \mathrm{~m}$ \\
\hline Pixel clock rate & $14.318 \mathrm{MHz}$ \\
\hline Sensing area & $7.6 \times 6.2 \mathrm{~mm}(1 / 2$ inch format $)$ \\
\hline Frame rate & 7.5 frames/second \\
\hline Digital image output & $\begin{array}{l}8 \text { bits } x 4 \text { taps or } 10 \text { bits } \times 3 \text { taps ( } 32 \text { bits } \\
\max \text { ), EIA-644, RS- } 422 \text {, or CameraLink }\end{array}$ \\
\hline Signal/Noise & $60 \mathrm{~dB}$ \\
\hline Electronic shutter & $\begin{array}{l}\text { Independent shutter time per channel } \\
\text { Range: } 1 / 8000-1 / 7.5 \mathrm{sec} \text {. }\end{array}$ \\
\hline Gain selection & Independent gain per channel 0-36 dB \\
\hline Offset selection & $\begin{array}{l}\text { Independent offset per channel } 0-127 \\
\text { counts }\end{array}$ \\
\hline Command/Control input & RS-232 port \\
\hline Operating voltage & $12 \mathrm{VDC}$ \\
\hline Power consumption & 10 Watts \\
\hline
\end{tabular}


Chapter 2 Methods for sclera patterns matching using high resolution multispectral images

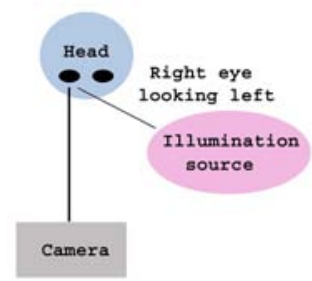

(a)

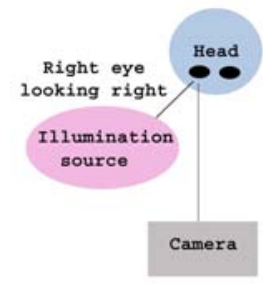

(b)

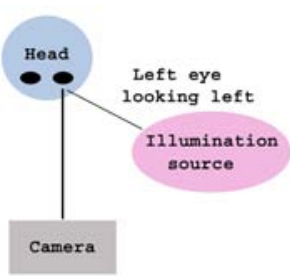

(c)

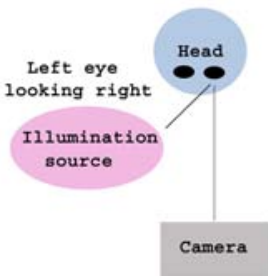

(d)

Figure 2.4 (a) Right-eye-looking-left $\left(R_{-} L\right)$. (b) Right-eye-looking-right $\left(R \_R\right)$. (c) Left-eye-looking-left $\left(L_{-} L\right)$. (d) Left-eye-looking-right $\left(L_{-} R\right)$.

While the person is gazing to the left or to the right, Figure 2.4, the camera can be easily manipulated to focus on the white of the eye. The light source (SL1-Filter, StellarNet Inc.) illuminates the eye using a spectral range from $350 \mathrm{~nm}$ to $1700 \mathrm{~nm}$, and is projected onto the eye via an optic fiber guide with a ring light attached to its end (HeiScope Annular Ring Light Guide). The amount of light is around 5.6 lux measured with cal-LIGHT 400L lightmeter (Cooke Corporation) at the temple, near the eye. Due to the reflective qualities of the eyeball, pointing a light source directly at the subject's eye creates a glare on the sclera. The issue is resolved by directing the light source such that the incoming rays to the eyeball are approximately perpendicular to the pupil region. This is not always possible due to subtle movements of the eyeball. Thus, glare is not always contained within the pupil region and may overlap with the iris.

The multispectral camera generates images with a resolution of $1040 \times 1392 \times 3$ pixels. The first 17 columns are removed due to artifacts. The final size of the images is $1035 \times 1373 \times 3$.

\section{Collection 1}

Videos of the right and left eye are captured from 103 subjects (Table 2.3), with 
Chapter 2 Methods for sclera patterns matching using high resolution multispectral images

each eye gazing to the right or to the left. Eight images per eye per gaze direction are selected from the video, based on a proper illumination, less specular reflection and in focus images. The total number of images is 3280 . For one subject, only data from the right eye was collected due to medical issues. Working with images from the same video allows us to bypass some of the challenges encountered by Crihalmeanu et al. [27] primarily due to viewing angle. The process of frame selection ensures that there is no remarkable change in pose. The camera is focused on the sclera region. Similarly the light is directed as much as possible towards the sclera region with the specular reflection as much as possible on the pupil. As a result in some images, mostly for left eye looking right $\left(L_{-} R\right)$ and right-eye-looking-left $\left(R_{-} L\right)$ the iris is less illuminated in the area close to the nose region.

\section{Collection 2}

Videos of the right and left eye are captured from 31 subjects (Table 2.3), with each eye gazing to the right or to the left. To increase the intra-class variation, the participant is asked to keep the head still and alternate the gaze direction between looking at the ring of lights (left or right side direction) and looking to the camera (frontal gaze direction) as illustrated in Figure 2.4. While gazing to the left or to the right, the subject is asked to look at a mark located on the ring of lights. Four images per eye per gaze direction are selected from the video when looking left or looking right, after each change in gaze direction from frontal to the side. The total number of images is 496 . The multispectral camera and the light are focused on the iris region. Due to the movement of the eye and the selection process of the frames, the four ocular images/eye/gaze have variations in viewing angle. Since the light is directed to the iris and due to the curvature of the eyeball, some images have sclera region less illuminated 
Chapter 2 Methods for sclera patterns matching using high resolution multispectral images

Table 2.3 High resolution multispectral database

\begin{tabular}{|c|c|}
\hline Collection 1 & Collection 2 \\
\hline 1 video/left eye & 1 video/left eye \\
\hline 1 video/right eye & 1 video/right eye \\
\hline Video 30 frames/sec & Video 30 frames/sec \\
\hline Camera focused on the sclera & Camera focused on the iris \\
\hline 8 consecutive images/eye/gaze & $\begin{array}{l}4 \text { images/eye/gaze (each image is } \\
\text { collected after the gaze direction is } \\
\text { changed from frontal to left or right) }\end{array}$ \\
\hline $\begin{array}{l}\text { Initial image size: } 1040 \times 1392 \times 3 \text {, } \\
17 \text { columns removed (artifacts), } \\
\text { Final image size: } 1035 \times 1373 \times 3\end{array}$ & $\begin{array}{l}\text { Initial image size: } 1040 \text { x } 1392 \text { x } 3 \text {, } \\
17 \text { columns removed (artifacts), } \\
\text { Final image size: } 1035 \times 1373 \times 3\end{array}$ \\
\hline 103 subjects & 31 subjects \\
\hline Total of 3280 images & Total of 496 images \\
\hline
\end{tabular}

in the corner of the eye opposite to lacrimal caruncle.

Both multispectral collections contain images of the eye with different iris colors. Based on the Martin-Schultz scale ${ }^{4}$, often used in physical anthropology, we classify the images as light eyes (blue, green gray), mixed eyes (blue, gray or green with brown pigment, mainly around the pupil) and dark eyes (brown, dark brown, almost black).

\subsubsection{From Bayer mosaic pattern to RGB}

The Bayer-like pattern [28] is due to the placement of a grid of tiny color filters on the face of the CCD sensor array to filter the light so that only one of the colors (red,

${ }^{4}$ http://wapedia.mobi/en/Eye_color 
Chapter 2 Methods for sclera patterns matching using high resolution multispectral images

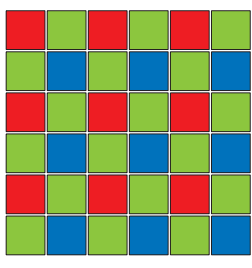

(a)

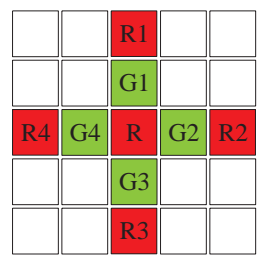

(b)

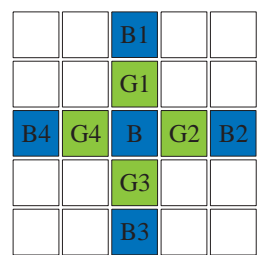

(c)

Figure 2.5 Bayer pattern: a) Bayer pattern grid. b) Green component, red pixel interpolation. c) Green component, blue pixel interpolation (Adapted from www.siliconimaging.com/RGB Bayer.htm).

blue or green) reaches any given pixel. Here, $25 \%$ of the pixels are assigned to blue, $25 \%$ to red and $50 \%$ to green. Blue and green components are obtained from the Bayer mosaic pattern through interpolation ${ }^{5}$. As illustrated in Figure 2.5, the value of the green component on a red pixel is interpolated according to the strength of the correlation on the vertical or horizontal direction of the neighboring red pixels:

$$
G(R)= \begin{cases}(G 1+G 3) / 2 & \text { if }|R 1-R 3|<|R 2-R 4| \\ (G 2+G 4) / 2 & \text { if }|R 1-R 3|>|R 2-R 4| \\ (G 1+G 2+G 3+G 4) / 4 & \text { if }|R 1-R 3|=|R 2-R 4|\end{cases}
$$

The green component is interpolated on a blue pixel as follow:

$$
G(B)= \begin{cases}(G 1+G 3) / 2 & \text { if }|B 1-B 3|<|B 2-B 4| \\ (G 2+G 4) / 2 & \text { if }|B 1-B 3|>|B 2-B 4| \\ (G 1+G 2+G 3+G 4) / 4 & \text { if }|B 1-B 3|=|B 2-B 4|\end{cases}
$$

The blue component values for green pixels are obtained in a similar way.

\footnotetext{
${ }^{5}$ RGB "Bayer" Color and MicroLenses, www.siliconimaging.com/RGB Bayer.htm
} 
Chapter 2 Methods for sclera patterns matching using high resolution multispectral images

\section{$2.2 \quad$ Image denoising}

The red, green, blue and NIR components obtained from the CIR images are in general noisy (Figure 2.6(a)(c)(e)(g)). The denoising algorithm employed is based on a wavelet transformation. A double-density complex discrete wavelet transform (DDCDWT) [29], which combines the characteristics and the properties of the doubledensity discrete wavelet transform (DDDWT) [30] and the dual-tree discrete wavelet transform (DTDWT) [1], is used. The transformation is based on two scaling functions and four distinct wavelets such that one pair of wavelets form an approximate Hilbert transform pair and the other pair of wavelets are offset from one other by one half. It is implemented by applying four 2-D double density discrete wavelet transforms in parallel to the input data with different filter sets for rows and columns, yielding 32 oriented wavelets (Figure 2.7(a)) along one of six angles at $\pm 15, \pm 45, \pm 75$ degrees ${ }^{6}$. The method is shift-invariant, possesses improved directional selectivity and is based on FIR perfect reconstruction filter banks as illustrated in Figure 2.7(b). For all scales and subbands, the magnitudes of the complex wavelet coefficients are processed by soft thresholding that sets the coefficients with values less than a threshold to zero and subtracts the threshold values from the non-zero coefficients. Original and denoised red, green, blue and NIR images are presented in Figure 2.6. Visual differences are not pronounced due to image rescaling. After denoising, all spectral components (NIR, red, green and blue) are geometrically resized by a factor of $1 / 3$ resulting in the final size of $310 \times 411$.

\footnotetext{
${ }^{6}$ http://taco.poly.edu/selesi/DoubleSoftware/index.html
} 
Chapter 2 Methods for sclera patterns matching using high resolution multispectral images

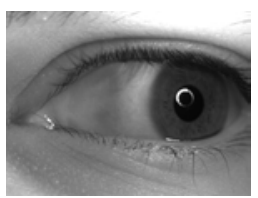

(a)

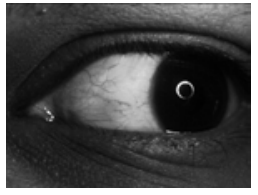

(e)

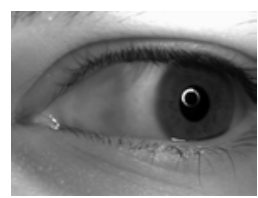

(b)

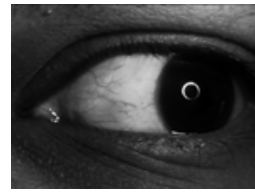

(f)

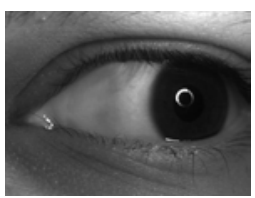

(c)

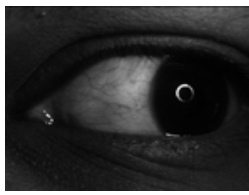

$(\mathrm{g})$

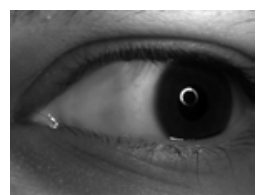

(d)

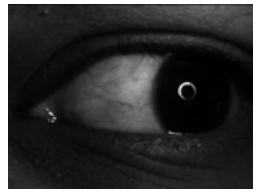

(h)

Figure 2.6 Denoising with Double Density Complex Discrete Wavelet Transform. a) Original NIR. b) Denoised NIR. c) Original red component. d) Denoised red component. e) Original green component. f) Denoised green component. g) Original blue component. h) Denoised blue component. Visual differences between original and denoised images are not pronounced due to image rescaling.

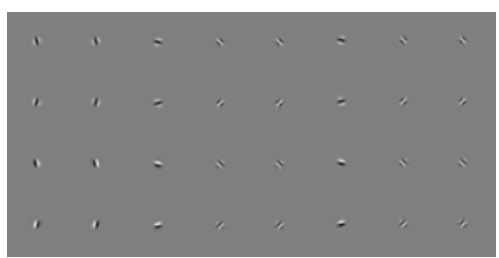

(a)

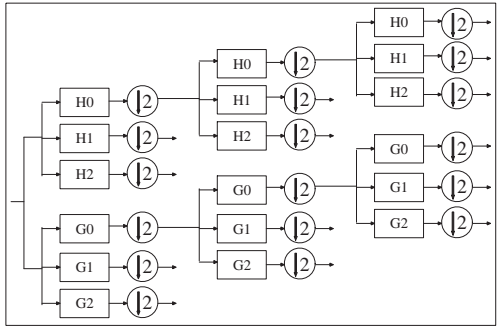

(b)

Figure 2.7 (a) Plot of Complex 2-D Double-Density Dual-Tree Wavelets. (b) Iterated filterbank for the Double-Density Complex Discrete Wavelet Transform [1]. 
Chapter 2 Methods for sclera patterns matching using high resolution multispectral images

\subsection{Specular reflection detection and removal}

Specular reflections have to be detected and removed as they can impact the sclera segmentation process (described in Section 2.4). The light directed to the eyeball generates specular reflection that has a ring-like shape, caused by the shape of the source of illumination, and highlights, due to the moisture of the eye and the curved shape of the eyeball. Both are detected and removed by a fast inpainting algorithm. In some images, the ring-like shape may be an incomplete circle, ellipse, or an arbitrary curved shape with a wide range of intensity values. It may be located partially in the iris region, making its detection and removal more difficult especially since the iris texture has to be preserved as much as possible. The specular reflections are detected using different intensity threshold values for each component: 0.60 for NIR, 0.50 for red and 0.80 for green. Only regions with less then 1000 pixels in size are labeled as specular reflection, are morphologically dilated and inpainted. In digital inpainting, the information from the boundary of the region to be inpainted is propagated smoothly inside the region. The value to be inpainted at a pixel is calculated using a PDE equation ${ }^{7}$ in which partial derivatives are replaced by finite differences between the pixel and its eight neighbors. Results are presented in Figure 2.8 .

\subsection{Sclera Region Segmentation}

When the entire image of the eye is used for enhancing the conjunctival vasculature, it is difficult to distinguish between the different types of lines that appear in it: wrinkles, crows feet, eyelashes, blood vessels. Therefore, a good segmentation of the sclera region that clearly exhibits the blood vessels is necessary. Even if the light is

\footnotetext{
${ }^{7}$ http://www.mathworks.com/matlabcentral/fileexchange/4551
} 
Chapter 2 Methods for sclera patterns matching using high resolution multispectral images

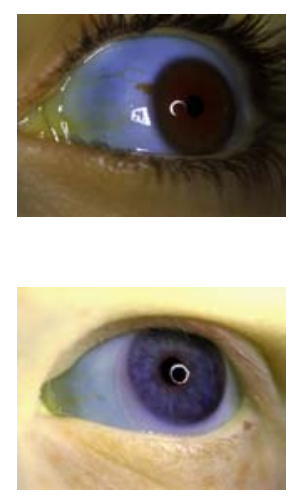

(a)
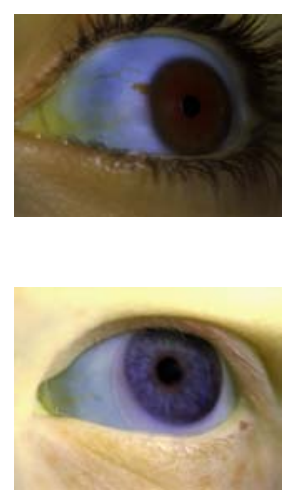

(b)

Figure 2.8 Specular reflection removal: (a) Original image. (b) Original image with specular reflection removed.

directed to the pupil region to avoid specular reflections, the curved nature of the eyeball presents a wide variety of intensity values across the sclera surface. Brighter skin regions as a result of illumination, and occasionally the presence of mascara, will make the segmentation of the sclera along the contour of the eyelid a challenging process. The algorithm to segment the sclera region has three main steps as described below.

\subsubsection{Coarse sclera region segmentation: The sclera-eyelid boundary}

The method employed to segment the sclera region along the eyelid contour is inspired by the work done in the processing of LandSat imagery (Land + Satellite) [31]. A set of indices are used to segment the vegetation regions in aerial multispectral images. Similarly, the index that we use for coarse sclera segmentation is based on the fact that the skin has lesser water content than the sclera, and hence exhibits a higher

reflectance in NIR. Since water absorbs NIR light, the corresponding regions based 
Chapter 2 Methods for sclera patterns matching using high resolution multispectral images
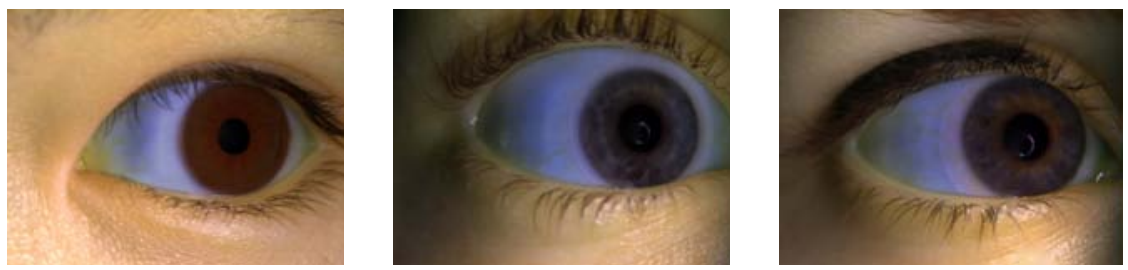

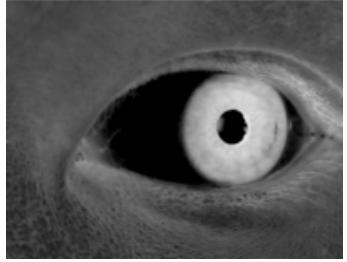

(a)

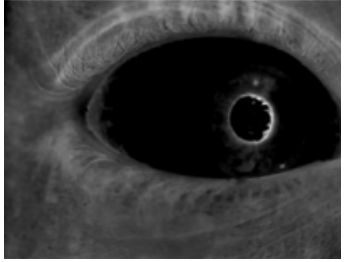

(b)

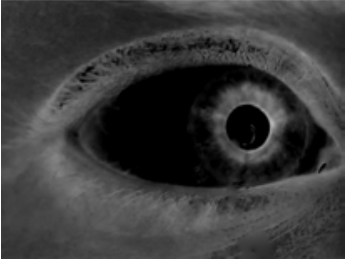

(c)

Figure 2.9 Sclera region segmentation. The first row displays the original image, the second row displays the normalized sclera index: (a) Dark colored iris. (b) Light colored iris. (c) Mixed colored iris.

on this index appear dark in the image. The algorithm is as follows:

1. Compute an index called the normalized sclera index $N S I(x, y)=\frac{N I R(x, y)-G(x, y)}{N I R(x, y)+G(x, y)}$, where $\operatorname{NIR}(x, y)$ and $G(x, y)$ are the pixel intensities of the NIR and green components, respectively, at pixel location $(x, y)$. The difference $N I R-G$ is larger for pixels pertaining to the sclera region; it is then normalized to help compensate for the uneven illumination. Figure 2.9 displays the normalized sclera index for all three categories as specified by the Martin-Schultz scale: light colored iris, dark colored iris and mixed colored iris.

2. Locate sclera by thresholding the NSI image with the threshold value $\eta=0.1$. Figure 2.10(a) displays the scatter plot between the NIR intensity values and the corresponding green intensity values for all pixels in the image. The pixels above the threshold $(\eta=0.1)$ represent the background region while the rest represent the sclera region. Changing the value of $\eta$ will modify the slope of the boundary line between the pixels of the two segmented regions. 
Chapter 2 Methods for sclera patterns matching using high resolution multispectral images
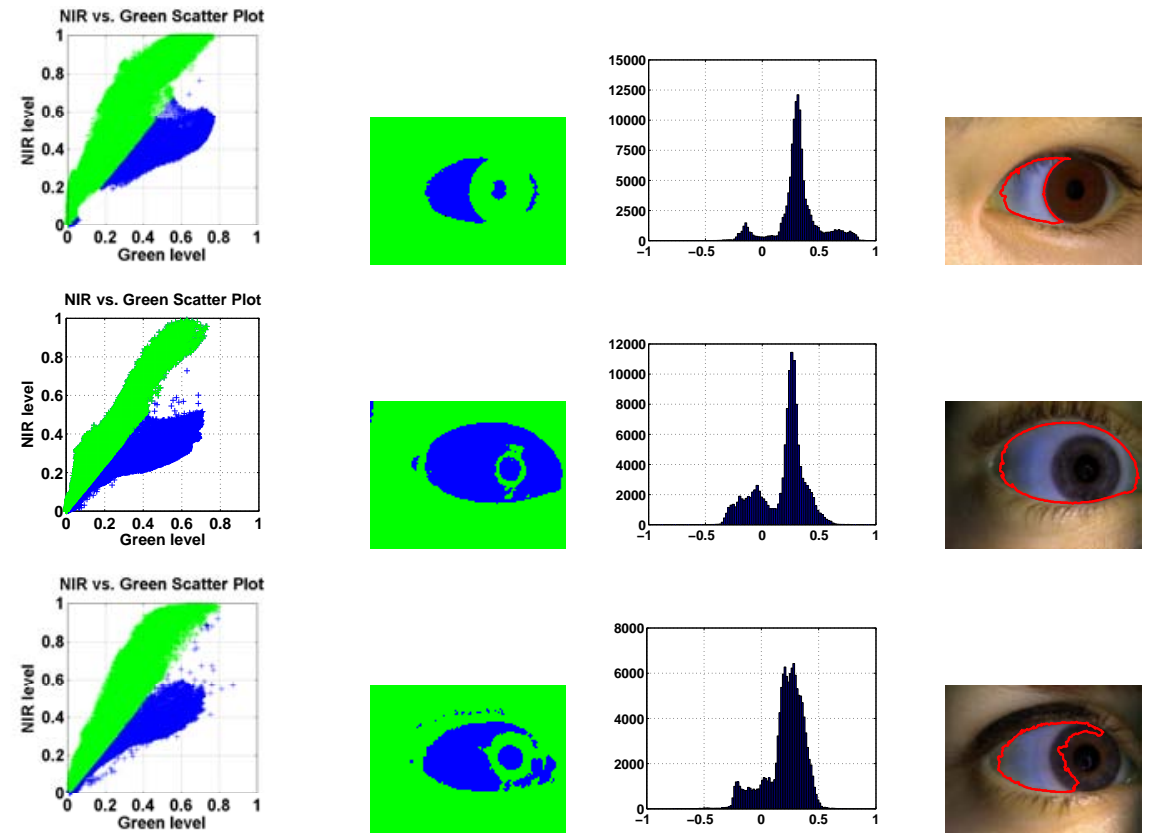

(a)

(b)

(c)

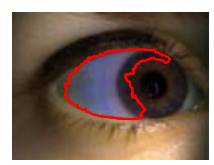

(d)

Figure 2.10 Sclera region segmentation. The first row displays the results for dark colored iris, the second row displays the results for light colored iris, and the third row displays the results for mixed colored iris: (a) NIR vs.green intensity values. (b) Threshold applied to NSI. (c) Histogram of the NSI. (d) Sclera mask contour imposed on original composite image. 
Chapter 2 Methods for sclera patterns matching using high resolution multispectral images

The output of the thresholding operation is a binary image ${ }^{8}$, Figure 2.10(b). For each category in the Martin-Schultz classification, the largest connected region in the binary image is composed of sclera region only; or the sclera and the iris; or the sclera and a portion of the iris. For dark irides (brown and dark brown), the sclera region excluding the iris is localized (Figure 2.10(d) the first row, referred henceforth as $I_{S}$ ). Thus, in this case, further segmentation of the sclera and iris is not required. For light irides (blue, green, etc.), regions pertaining to both the sclera and iris are segmented (Figure 2.10(d) the second row, referred henceforth as $I_{S}$ ). Here, further separation of the sclera and iris is needed. For mixed irides (blue or green with brown around pupil), the region of the sclera and the light colored portion of the iris are segmented as one region (referred henceforth as $I_{S}$ ). The dark portion of the iris (brown) is not included (Figure 2.10(d) the third row). Here, further separation of the sclera and the portion of the iris is needed. To finalize the segmentation of the sclera, i.e., to find the boundary between the sclera and the iris regardless of the color of the iris, the pupil is detected. The convex hull of the segmented region $I_{S}$ and the pupil region will contain the sclera, the pupil, and the iris or the portion of the iris. This region is referred to as $I_{S I P}$ and is further processed. Since the proposed algorithm does not deduce the color of the iris, it is applicable to all images irrespective of the eye color. As seen in Figure 2.10(b), the location of the pupil is also visible either as a dark region that does not overlap the sclera region (in dark and mixed irides) or as a lighter disk within the sclera region (in light irides). This information can be exploited only if the color of the iris is known in advance. Therefore, in Section 2.4.2 we present an automatic way

\footnotetext{
${ }^{8}$ MathWorks, Image Processing Toolbox, Finding Vegetation in a Multispectral Image, http://www.mathworks.com/products/image/demos.html
} 
Chapter 2 Methods for sclera patterns matching using high resolution multispectral images

of finding the pupil location regardless of the color of the iris.

\subsubsection{Pupil region segmentation}

The location of the pupil is needed to determine $I_{S I P}$ and to find the boundary between the sclera and the iris regardless of the color of the eye. Hence, the accurate determination of its boundary is not necessary. In NIR images, the pupil region is characterized by very low intensity values and, by employing a simple threshold, the pupil region is obtained. However, this isolates the eyelashes as well. In order to isolate only the pupil, the following steps are undertaken:

1. Geometrically resize the NIR component by a factor of $1 / 3$ and apply power-law transformation [21] to its pixels: $I_{P L}=c * I_{I}^{x}$, where $c=1$ is a constant, $I_{P L}$ is the output image, $I_{I}$ is the input NIR image and $x=0.7$.

2. Threshold $I_{P L}$ with a value of 0.1 . The resulting binary image, $I_{B W}$, has the pupil and eyelashes denoted by 1 .

3. Find the contour of the convex hull of the sclera region as segmented in Section 2.4.1, $I_{S C H}$, Figure $2.11(\mathrm{~b}),(\mathrm{d})$.

4. Use Hough transform for line detection. Select and remove the highest peak corresponding to the longest line, Figure 2.11 (c), (d).

5. Fit an ellipse to the remaining sclera contour points, $E\left(a, b,\left(x_{0}, y_{0}\right), \theta\right)$, where $a$, $b,\left(x_{0}, y_{0}\right)$ and $\theta$ correspond to the length of the semi-major axes, length of the semi-minor axes, the center of the ellipse, and its orientation, respectively Figure 2.11 (e). Define an elliptical mask (to detect the pupil region) to extract the pixels located within the ellipse. 
Chapter 2 Methods for sclera patterns matching using high resolution multispectral images

6. Impose the ellipse mask on the binary image $I_{B W}$ obtained in step 2 . The result is a binary image that will contain the pupil, and possibly eyelashes, as logical 1 pixels, $I_{P}$.

7. Count the number of connected objects $\mathrm{N}$ in $I_{P}$. If $N>1$, through an iterative process, decrease the ellipse's semi-major and semi-minor axis (by 2\%) and construct new elliptical masks that when imposed on the binary image $I_{B W}$ will render a smaller value for $\mathrm{N}$. The connected object for $N=1$ will correspond to the location of the pupil.

$$
\begin{aligned}
& \text { while } N>1 \text { do } \\
& \begin{array}{l}
a=a-\frac{2}{100} \times a, \\
b=b-\frac{2}{100} \times b, \\
E_{M A S K}=E\left(a, b,\left(x_{0}, y_{0}\right), \theta\right) \\
I_{P}=I_{B W} \cap E_{M A S K} \\
\text { find } N \text { in } I_{P}
\end{array} \\
& \text { end while }
\end{aligned}
$$

8. Fit a new ellipse $E$ to the dilated region corresponding to the location of the pupil. Compute $I_{P}=I_{B W} \cap E_{M A S K}$. Even if low intensity regions in the iris are inadvertently selected, the pupil region has by far the largest area among all connected objects.

9. Fit an ellipse to the pixels pertaining to the pupil region to find the pupil mask, $P_{\text {MASK }}$. Resize the pupil mask to the original NIR image size Figure 2.11 (f).

The procedure described above is applied to all the images regardless of the color of the iris. For 15 images, the algorithm failed to correctly segment the pupil. 
Chapter 2 Methods for sclera patterns matching using high resolution multispectral images
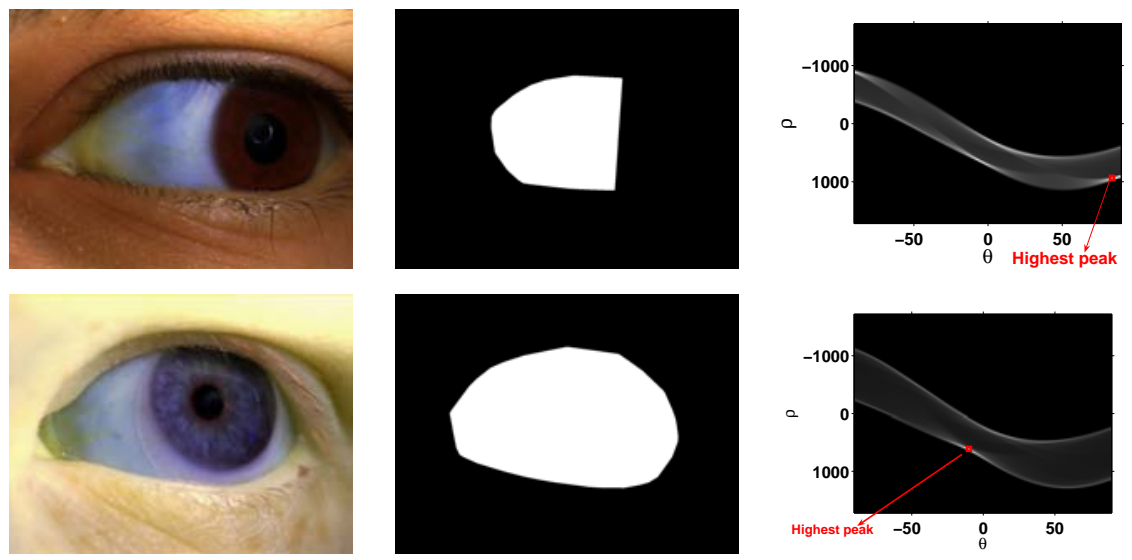

(a)

(b)

(c)
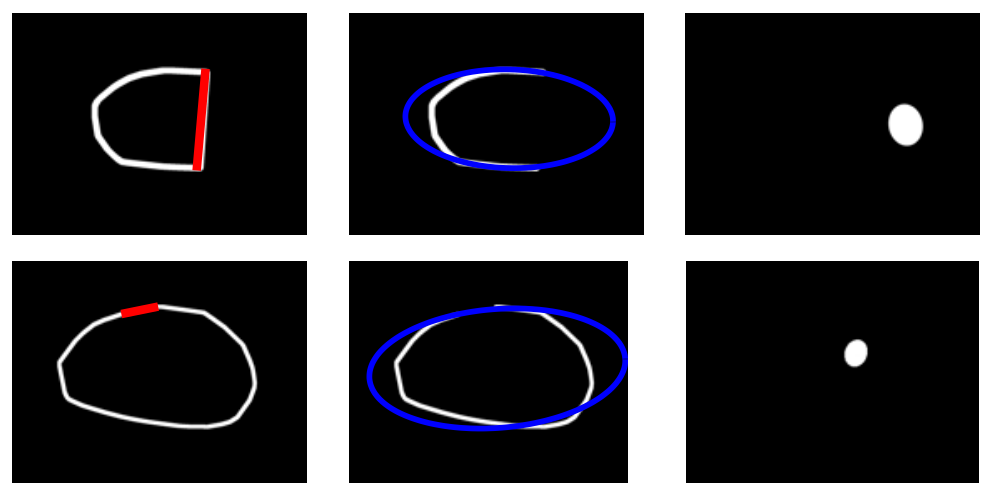

(d)

(e)

(f)

Figure 2.11 Pupil region segmentation. The first row displays the results for dark colored iris, the second row displays the results for light colored iris: (a) Original image. (b) Convex hull of the sclera region. (c) Hough transform and the highest peak. (d) Sclera region contour and the longest line. (e) The ellipse fitted to the sclera contour. (f) Output of the pupil segmentation algorithm. 
Chapter 2 Methods for sclera patterns matching using high resolution multispectral images
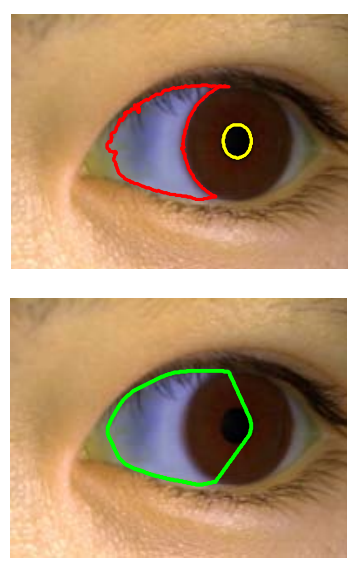

(a)
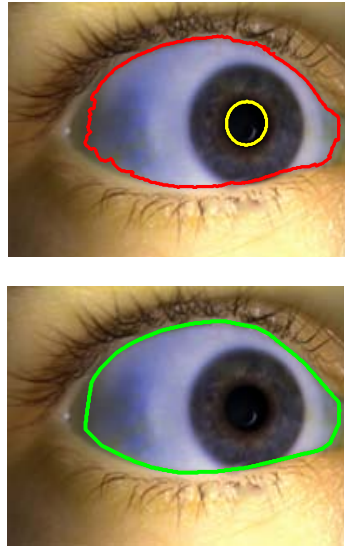

(b)
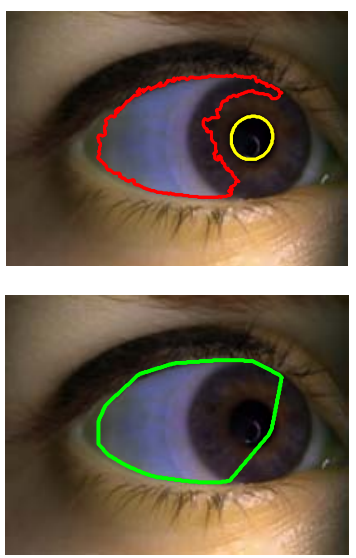

(c)

Figure 2.12 Sclera region segmentation. The first row displays the contour of the sclera cluster and pupil mask, the second row displays the Contour of the convex hull of the sclera cluster and pupil mask imposed on the composite image $I_{S I P}$. (a) Dark colored iris. (b) Light colored iris. (c) Mixed colored iris.

\subsubsection{Fine sclera region segmentation: The sclera-iris bound- $\operatorname{ary}$}

As mentioned in Section 2.4.1, the convex hull $I_{S I P}$, of the segmented region $I_{S}$ and the pupil region (Figure 2.12 second row) will contain the sclera, the pupil and the iris or the portion of the iris. A finer segmentation of the iris is needed regardless of the color of the eye. As in [32], we define four measures called "proportion of sclera" $p(x, y)$ in four directions: north, south, west and east. In $I_{S I P}$, the value of $p(x, y)$ is set to 0 for all the pixels outside the convex hull region. For a pixel $(x, y)$ inside the convex hull, the proportion of sclera in the north direction, $p_{\uparrow}(x, y)$, is computed as the mean of all the pixels of column $y$ above the location $(x, y)$. The proportion of sclera in the south direction, $p_{\downarrow}(x, y)$, is computed as the mean of all the pixels of column $y$ below the location $(x, y)$. The proportion of sclera in the west direction, $p_{\leftarrow}(x, y)$, is computed as the mean of all the pixels along row $x$, left of the location 
Chapter 2 Methods for sclera patterns matching using high resolution multispectral images
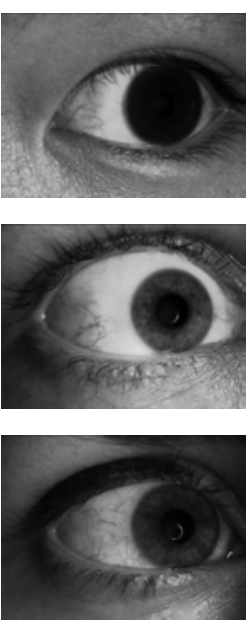

(a)
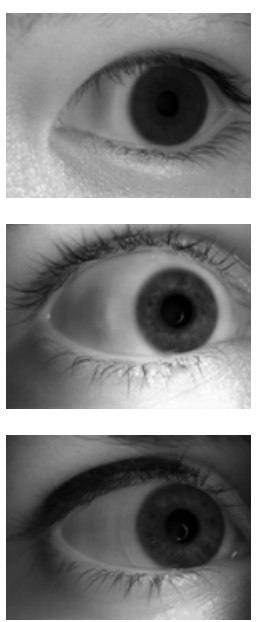

(b)
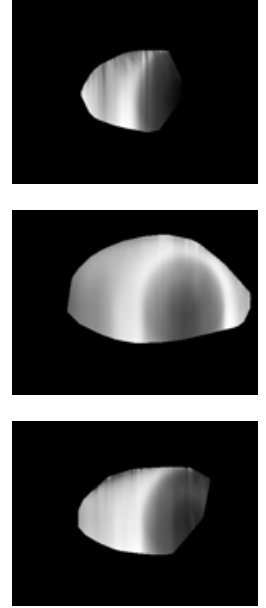

(c)
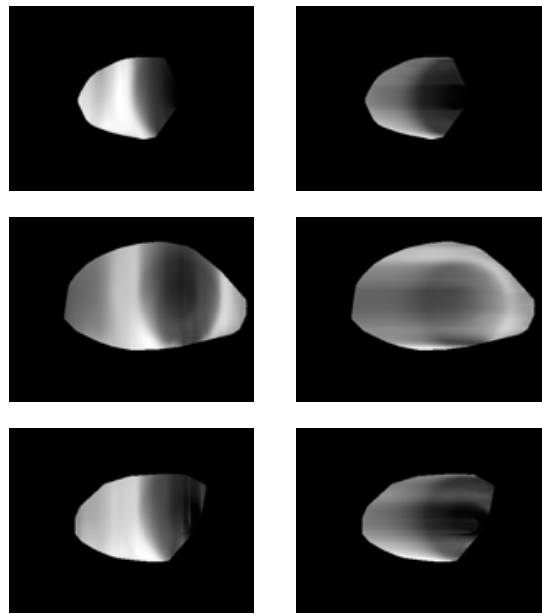

(d)
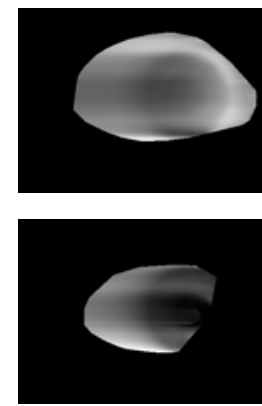

(e)

Figure 2.13 Sclera region segmentation. The first row displays the results for dark colored iris, the second row displays the results for light colored iris, and the third row displays the results for mixed colored iris: (a) Green component. (b) Red Component. (c) Proportion of sclera in north direction $p_{\uparrow}(x, y)$. (d) Proportion of sclera in south direction $p_{\downarrow}(x, y)$. (e) The proportion of sclera in east direction $p_{\leftarrow}(x, y)$ for left gaze direction.

$(x, y)$ and the proportion of sclera in the east direction, $p_{\rightarrow}(x, y)$, is computed as the mean of all the pixels along row $x$, right of the location $(x, y)$. Figure 2.13 (c)-(e) illustrates this procedure.

We use the $\mathrm{k}$-means clustering algorithm $(\mathrm{k}=2)$ to segment the iris, and find the limbus (sclera-pupil) boundary. The algorithm uses the pixels contained within the segmented region $I_{S I P}$ as its input. Each pixel is viewed as a five-dimensional entity consisting of the intensity value of the green component, intensity value of the red component, the proportion of sclera in the north $p_{\uparrow}(x, y)$ and south directions $p_{\downarrow}(x, y)$. According to the gaze direction - looking-to-the-left or looking-to-the-right - the proportion of the sclera in the west or east direction as assessed in the red component is used as the fifth feature. To detect the direction of the gaze, the $y$ coordinate of the centroid of the segmented region $I_{S}$ and the centroid of the pupil region is found 
Chapter 2 Methods for sclera patterns matching using high resolution multispectral images

and compared. For the left gaze direction $y_{\text {pupil }}>y_{\text {sclera }}$ and proportion of sclera in the east direction is used; for the right gaze direction $y_{\text {pupil }}<y_{\text {sclera }}$ and the proportion of sclera in the west direction is used. Euclidean distances between the origin of the coordinate system and the centroid of each cluster are computed in order to determine the label of the two clusters (the label can be 'sclera' or 'iris'). The largest distance is associated with the sclera cluster; this is the white region in Figure 2.14 first row. The smallest distance is associated with the iris cluster; this is the black region in Figure 2.14 first row. Two binary images, a mask for the sclera region and a mask for the iris region represent the output. On examining the two binary masks, we observe that in some images, the k-means algorithm erroneously labels portion of the sclera as being the iris (mainly the corners of the sclera that are less illuminated and have lower intensity values). To address this issue, if the iris mask has more than one connected region, the region in the iris mask that overlaps with the pupil mask is assumed to be the iris region, and is subtracted from the convex hull $I_{S I P}$.

The algorithm failed to segment the sclera region properly for a total number of 151 images. This is due to improper illumination and plenty of mascara (Figure 2.15) present in some images. The pupil segmentation algorithm finds the convex hull of the sclera region. This method creates straight lines along the contour of the sclera. For dark colored irides and mix colored irides, the longest induced line that is to be removed using Hough transform (Section 2.4.2) connects the highest point with the lowest point on the curved boundary of the iris and sclera (Figure 2.16 (a), the red line). It may happen that the longest line induced by the convex hull is not the proper one, but one that is located along the sclera contour (Figure 2.16 (b)). As a result, after removal of the line with Hough transform, the fitted ellipse to the contour of the sclera will no longer generate the elliptical mask needed. 
Chapter 2 Methods for sclera patterns matching using high resolution multispectral images
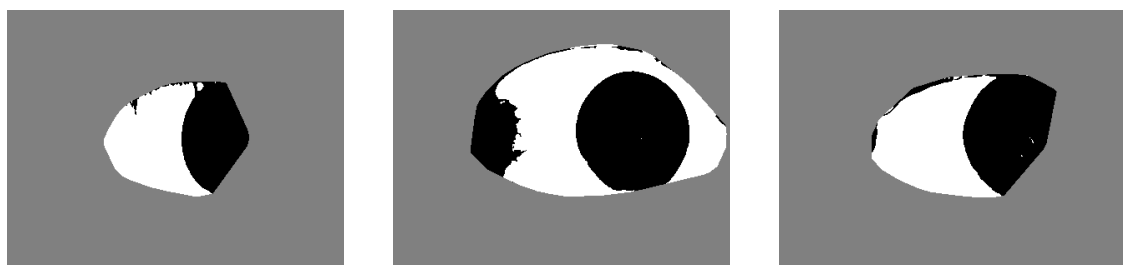

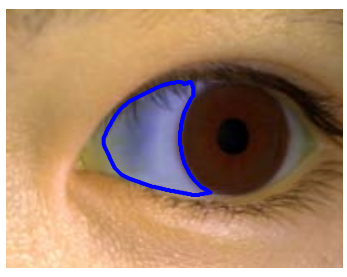

(a)

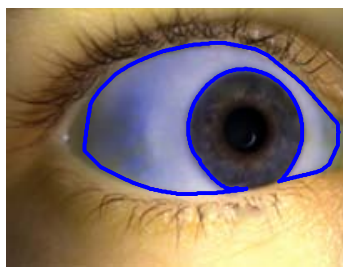

(b)

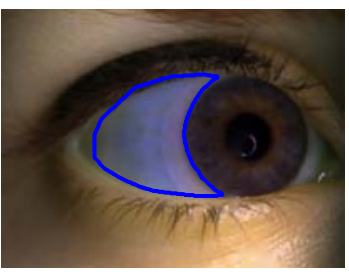

(c)

Figure 2.14 Sclera region segmentation. The first row displays the K-means output, the second row displays Contour of the segmented sclera mask imposed on the composite image: (a) Dark colored iris. (b) Light colored iris.(c) Mixed colored iris.

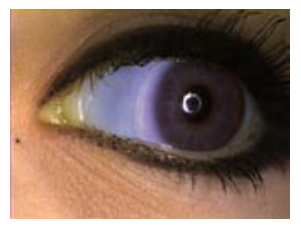

(a)

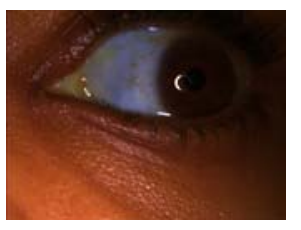

(b)

Figure 2.15 Example of eye images with: (a) Plenty of mascara. (b) Improper illumination. 
Chapter 2 Methods for sclera patterns matching using high resolution multispectral images

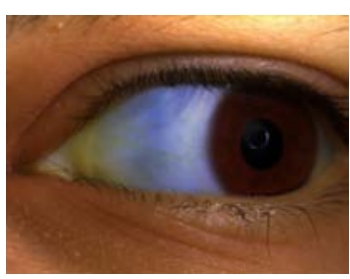

(a)

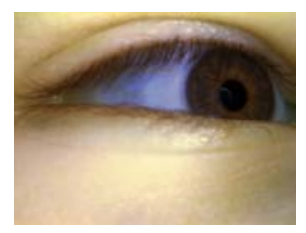

(b)
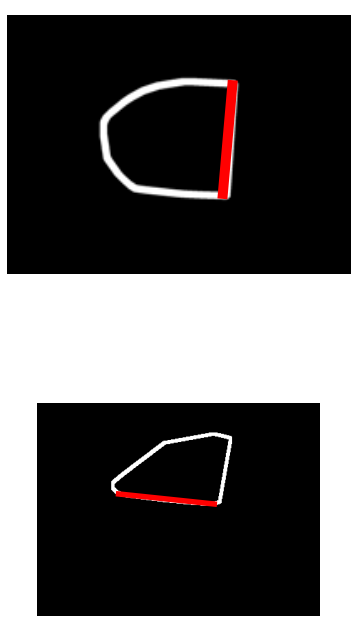

Figure 2.16 Failure to remove the proper line using Hough transform: (a) Correct detection of the longest line. (b) Incorrect detection of the longest line.

\subsection{Enhancement of blood vessels observed on the sclera}

An examination of the three components of the RGB image, suggests that the green component has the best contrast between the blood vessels and the background. To improve segmentation of the blood vessel patterns, the green component of the segmented sclera image is pre-processed using a selective enhancement filter for lines as described in [33] and similarly used in [27]. The enhancement filter for lines, and implicitly for blood vessels, is described by the equation:

$$
I_{\text {line }}\left(\lambda_{1}, \lambda_{2}\right)=\left\{\begin{array}{rll}
\left|\lambda_{1}\right|-\left|\lambda_{2}\right| & \text { if } & \lambda_{1}<0 \\
0 & \text { if } & \lambda_{1} \geq 0
\end{array}\right.
$$

where $\lambda_{1}$ and $\lambda_{2}$ (with $\left|\lambda_{1}\right|>\left|\lambda_{2}\right|$ ) are the two eigenvalues of the Hessian matrix of each pixel and computed as follows: $\lambda_{1}=K+\sqrt{\left(K^{2}-Q^{2}\right)}, \lambda_{2}=K-\sqrt{\left(K^{2}-Q^{2}\right)}$, 
Chapter 2 Methods for sclera patterns matching using high resolution multispectral images

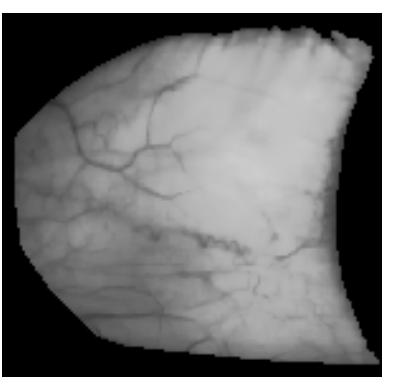

(a)

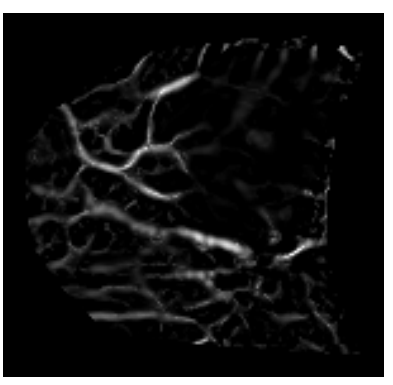

(b)

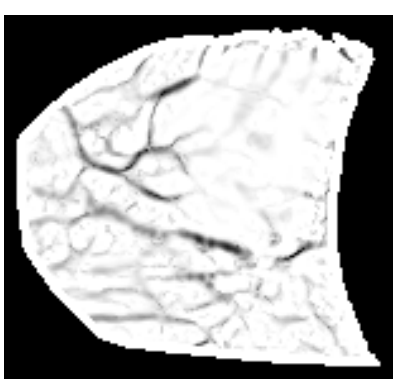

(c)

Figure 2.17 Blood vessel enhancement on the segmented sclera region. (a) Green component of the segmented sclera. (b) Result of the enhancement of blood vessels. (c) The complement image of the enhanced blood vessels

where $K=\left(I_{x x}+I_{y y}\right) / 2, Q=\sqrt{\left(I_{x x} * I_{y y}-I_{x y} * I_{y x}\right)}, I_{x x}, I_{y y}, I_{x y}$ and $I_{y x}$ represent the second-order derivatives in $\mathrm{x}$ and $\mathrm{y}$ directions. The algorithm for blood vessels enhancement described in [33] is as follows:

1. Determine the minimum $\left(d_{\min }\right)$ and maximum $\left(d_{\max }\right)$ diameter of the blood vessels

2. Consider multiple 2D Gaussian distributions (N) with standard deviation within the interval $\left[d_{\min } / 4, d_{\max } / 4\right]$.

3. Convolve each Gaussian distribution with the original image

4. Compute the two eigenvalues for each pixel, for each of the $\mathrm{N}$ convolved images.

5. Using the eigenvalues, compute $I_{\text {line }}$.

6. Multiply each pixel with the square of the corresponding Gaussian standard deviation, $I_{\text {line }} * \sigma^{2}$.

7. Consider the maximum value at each pixel level from all $\mathrm{N}$ outputs: $I_{\text {out }}=$ $\operatorname{argmax}\left(I_{\text {line }} * \sigma^{2}\right)$. 
Chapter 2 Methods for sclera patterns matching using high resolution multispectral images

\subsection{Image registration}

In this method, the two images to be compared are first registered using an image alignment scheme, and a direct correlation between corresponding pixels is then used to determine their similarity. Image registration is the process of finding a transformation that aligns one image with another. The regions of the sclera in the two images that are to be registered are cropped, and the images are padded to the same size. The image with the smaller height is padded up and down with an equal number of rows. If the gaze direction of the eye is to the left, the image with the smaller width is padded to the right. If the gaze direction of the eye is to the right, the image with the smaller width is padded to the left. To detect the direction of the gaze, the $y$ coordinate of the centroid of the sclera region and the centroid of the pupil region is found and compared. This process results in a better overlap of the two sclera regions. The registration method used here, described in [34], models a local affine and a global smooth transformation. It also accounts for contrast and brightness variations between the two images that are to be registered. The registration between two images, the source $I(x, y, t)$ and the target $I(\hat{x}, \hat{y}, t-1)$, is modeled by the transformation $\vec{m}=\left(m_{1}, m_{2}, m_{3}, m_{4}, m_{5}, m_{6}, m_{7}, m_{8}\right)$ : $m_{7} I(x, y, t)+m_{8}=I\left(m_{1} x+m_{2} y+m_{5}, m_{3} x+m_{4} y+m_{6}, t-1\right)$, where $m_{1}, m_{2}, m_{3}$, and $m_{4}$ are the linear affine parameters, $m_{5}, m_{6}$ are the translation parameters, and $m_{7}, m_{8}$ are the contrast and brightness parameters. A multi-scale approach is employed by using a Gaussian pyramid to downsample the images to be registered. From a coarseto-fine level, the transformation $\vec{m}$ is determined globally at each level, and then locally, and the estimated parameters are used to warp the source image. Using the linear affine parameters $m_{1}, m_{2}, m_{3}$, and $m_{4}$, and the translation parameters $m_{5}, m_{6}$, the sclera mask of the source image is also registered. Figure 2.18 shows results for 
Chapter 2 Methods for sclera patterns matching using high resolution multispectral images

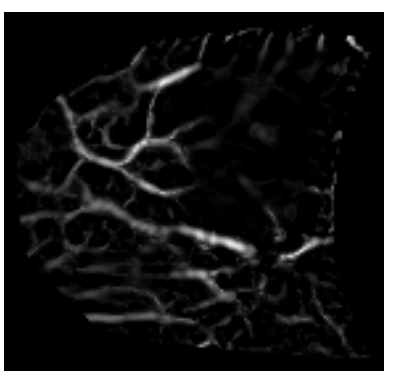

(a)

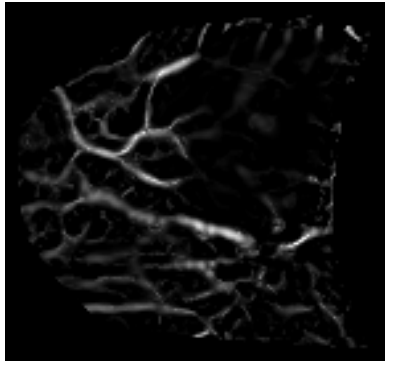

(b)

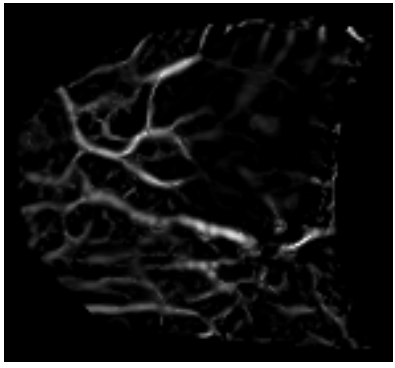

(c)

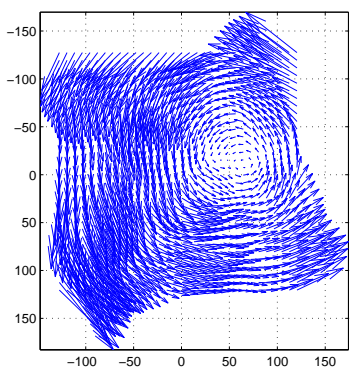

(d)

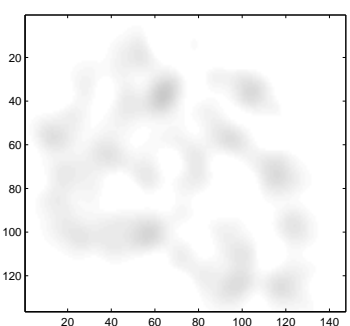

(e)

Figure 2.18 Image registration of the sclera region from images of the same eye. a) Source image. b) Target image. c) Registered source. d) Flow image depicting the warping process. e) Estimated contrast map

the registration of two pre-processed sclera images of the same eye. Figure 2.19 shows results for the registration of two pre-processed sclera images of different eyes.

\subsection{Feature extraction and matching}

The algorithms used to compare two images may consider the entire image, such as the pixel intensity or may rely on the characteristic features extracted from the images. These features have to be detectable under changes in image scale, noise and illumination. The design of three different feature extraction and matching methods is presented. The first one is based on interest-point detection and utilizes the entire sclera region including the vasculature pattern; the second is based on minutiae points 
Chapter 2 Methods for sclera patterns matching using high resolution multispectral images

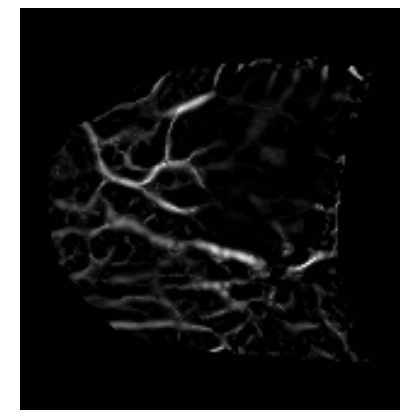

(a)

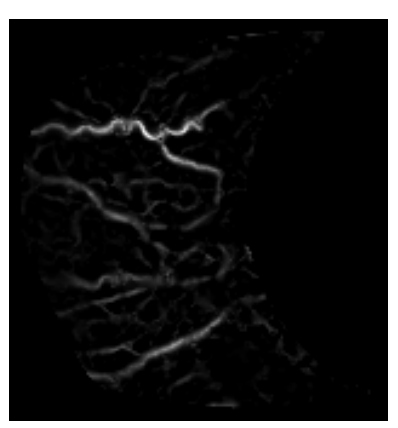

(b)

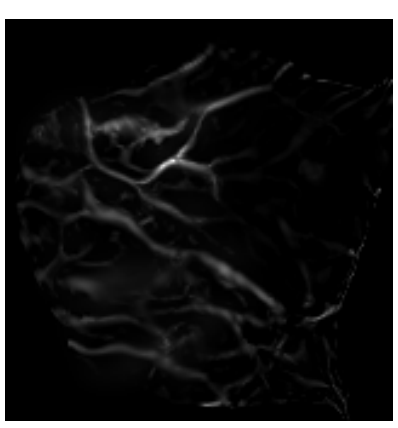

(c)

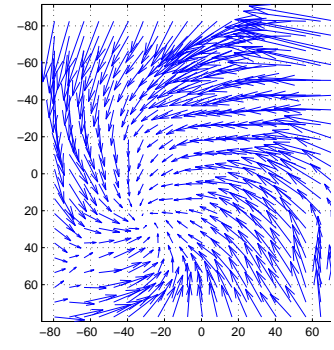

(d)

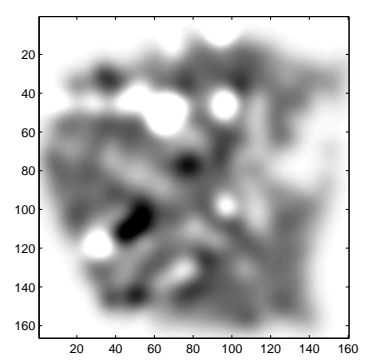

(e)

Figure 2.19 Image registration of the sclera region from two different eyes. a) Source image. b) Target image. c) Registered source. d) Flow image depicting the warping process. e) Estimated contrast map. 
Chapter 2 Methods for sclera patterns matching using high resolution multispectral images

on the vasculature structure; and the third is based on direct correlation. While the first two techniques do not need an explicit image registration scheme, the third technique relies on image registration.

\subsubsection{Speeded Up Robust Features (SURF)}

The Speeded-Up Robust Features (SURF) algorithm [35] is a scale and rotation invariant detector and a descriptor of point correspondences between two images. These points called "interest points" are prominent structures such as corners and T-junctions on the image. The algorithm uses a detector to locate interest points that are represented using a feature descriptor. The detector employs a Hessian matrix applied to the image convolved with Laplacian of Gaussian filters that further are approximated as box filters. These approximations allow the use of integral images for image convolution as described in [36]. The scale space is divided into octaves and is analyzed by up-scaling the filter size. The same image is convolved with a filter of increasing size at a very low computational cost. Interest points over multiple scales are localized using a non-maximum suppression algorithm as described in [37]. The localized maximums are interpolated in scale and image space using [38]. The descriptor uses the distribution of intensity values in a square region of size equal to $20 s$, where $s$ is the scale centered at the interest point. This region is further split into sub-regions. The entries for the 64 length feature vector are the sum of the Haar wavelet responses on the horizontal and vertical directions in these sub-regions. In our work, SURF is applied to the enhanced blood vessel images. The similarity between two images is assessed using the Euclidean distance as a measure between their respective corresponding interest points. Only Euclidean distances greater than 0.1 are considered and the number of corresponding interest point pairs is counted. Figure 2.20 displays the corresponding interest points between images of the same 
Chapter 2 Methods for sclera patterns matching using high resolution multispectral images

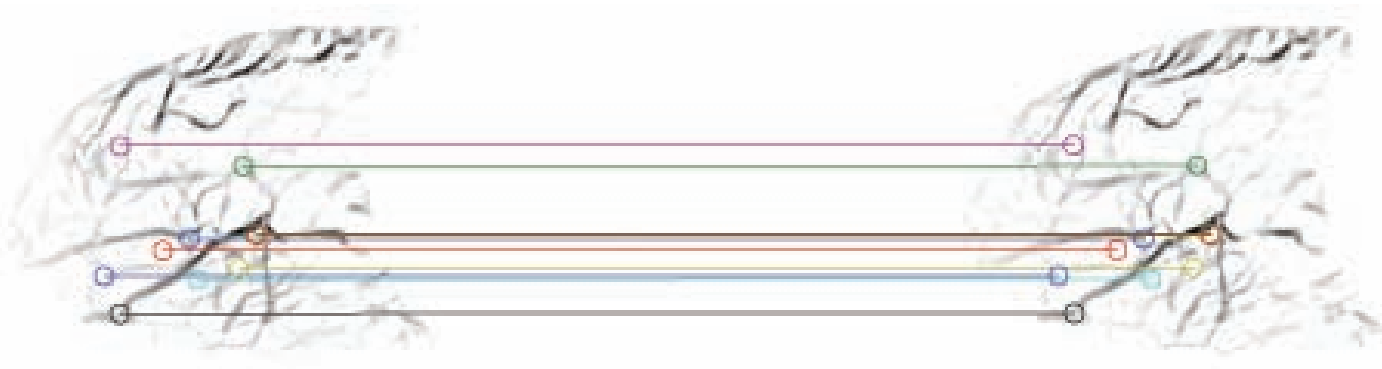

(a)

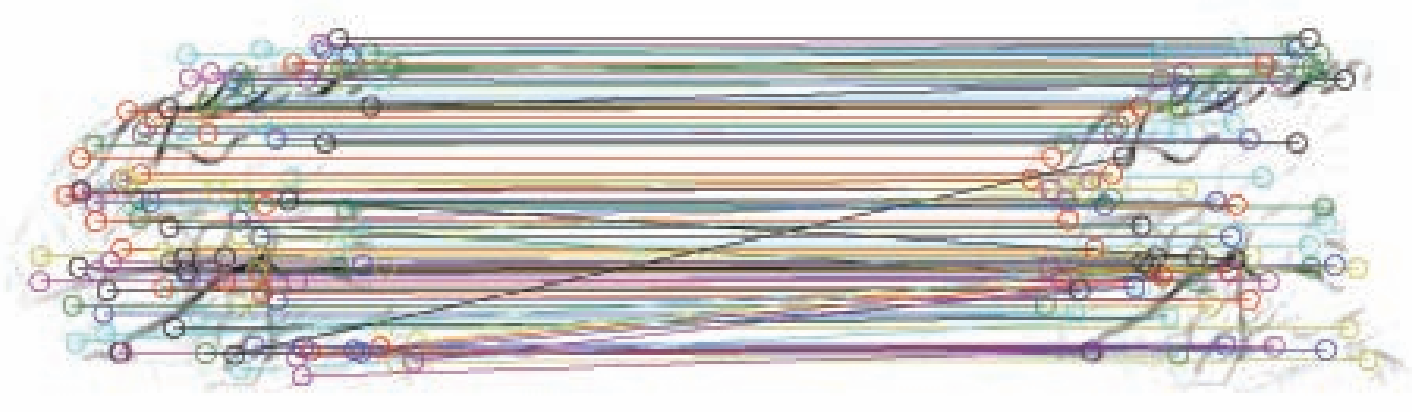

(b)

Figure 2.20 The output of the SURF algorithm when applied to enhanced blood vessel images of the same eye (The complement of the enhanced blood vessel images are displayed for better visualization). The number of interest points: 112 and 108. (a) The first 10 pairs of corresponding interest points. (b) All the pairs of corresponding interest points.

eye, and Figure 2.21 between images of two different eyes.

\subsubsection{Minutiae detection}

Another technique to represent and match scleral images is based on the cross-over points of the conjunctival vasculature. These are referred as minutiae points based on the fingerprint biometric literature [39]. Because of the large variations in intensity values and the low contrast between the blood vessels and the background, classical methods of segmentation based on edge detection are not robust and do not give good results. The region growing method is used for segmenting the enhanced blood vessels 
Chapter 2 Methods for sclera patterns matching using high resolution multispectral images

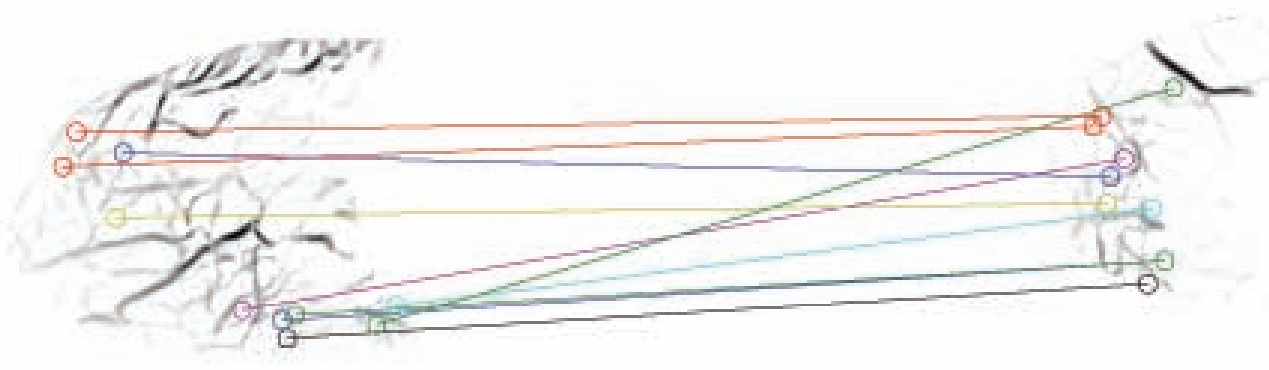

(a)

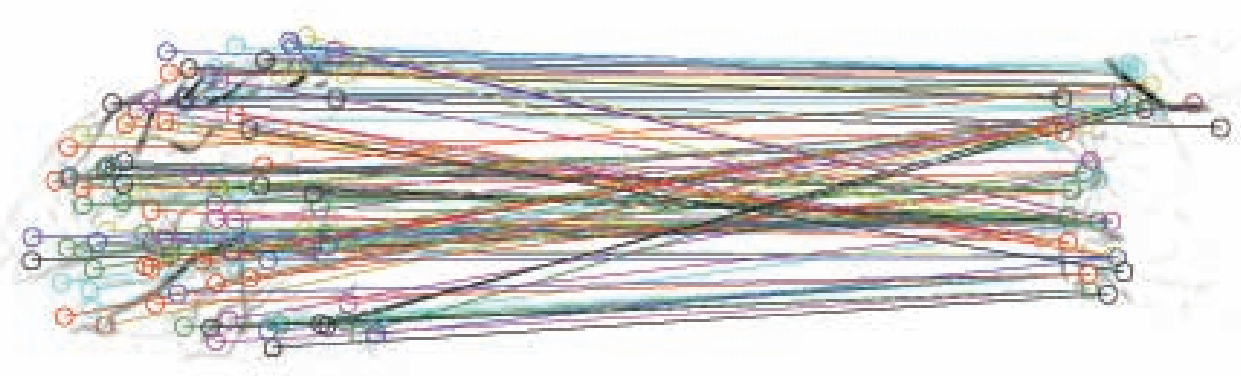

(b)

Figure 2.21 The output of the SURF algorithm when applied to enhanced blood vessel images of different eyes (The complement of the enhanced blood vessel images are displayed for better visualization). Number of interest points: 112 and 64. (a) The first 10 pairs of corresponding interest points. (b) All the pairs of corresponding interest points. 
Chapter 2 Methods for sclera patterns matching using high resolution multispectral images

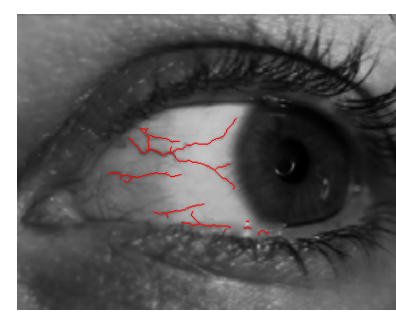

(a)

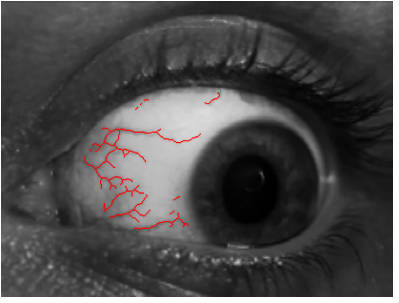

(b)

Figure 2.22 The centerline of the segmented blood vessels imposed on the green component of two images.

based on the algorithm described in [40]. The labeling of each pixel as pertaining to the conjunctival vasculature or background, is based on the information provided by the intensity value and the magnitude of the gradient of the pre-processed image. The result of conjunctival vasculature segmentation using region growing is a binary image, that is subjected to morphological operations, mainly a thinning procedure through which the blood vessel thickness is reduced to one pixel (Figure 2.23 (b)).

The minutiae points, in this work, correspond to the bifurcations of the centerline of the blood vessels (Figure 2.23 (c). Each blood vessel ramification has to be as least 4 pixels in length. A point matching algorithm is used to compare the points extracted from two images where each point is characterized as a $(x, y)$ location. The matching algorithm consists of finding an alignment between the two sets of points that will result in the maximum overlap of minutiae pairs from the two images. For two minutiae sets, $A=\left\{a_{1}, a_{2}, a_{3}, \ldots a_{m}\right\}, a_{i}=\left(x_{i}, y_{i}\right), i=1 . . m$ and $B=\left\{b_{1}, b_{2}, b_{3}, \ldots b_{n}\right\}, b_{j}=\left(x_{j}, y_{j}\right), j=1 . . n$, where $m$ and $n$ are the number of minutiae in $A$ and $B$, minutiae $a_{i}$ is said to be in correspondence with a minutiae $b_{j}$ if the Euclidean distance (E) between them is smaller than a given tolerance $t$, i.e., $E\left(a_{i}, b_{j}\right)=\sqrt{\left(\left(x_{j}-x_{i}\right)^{2}+\left(y_{j}-y_{i}\right)^{2}\right)} \leq t$. The match score is computed as the square of the number of corresponding minutiae points divided by the product $m n$. The algorithm failed to detect minutiae points on 28 images. This is due to the de- 
Chapter 2 Methods for sclera patterns matching using high resolution multispectral images

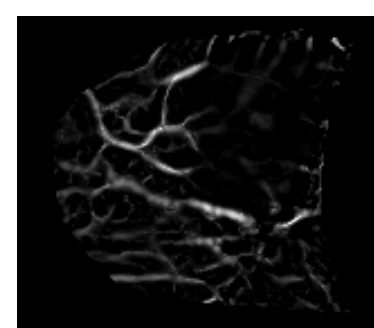

(a)

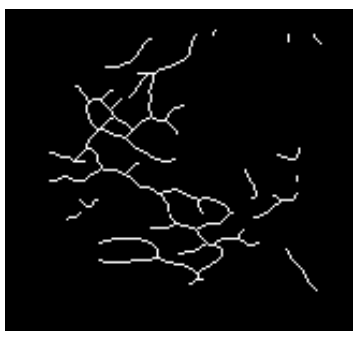

(b)

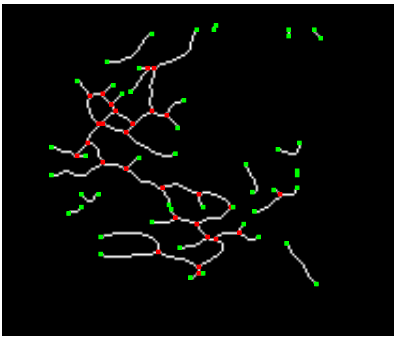

(c)

Figure 2.23 Detection of minutiae points. (a) Enhanced blood vessels image. (b) Centerline of the detected blood vessels. (c) Minutiae points: bifurcations (red) and endings (green).

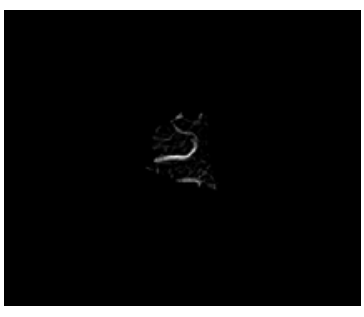

(a)

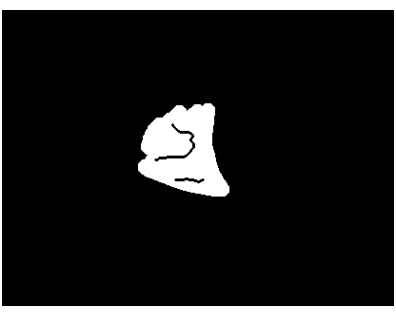

(b)

Figure 2.24 Failure to detect minutiae points. (a) Enhanced blood vessels image. (b) The detected vasculature without ramifications and intersections (Morphological operations such as dilation is applied to the blood vessels for a better visualization).

tection of blood vessels without ramifications and blood vessels that do not intersect (Figure 2.24).

\subsubsection{Direct correlation}

Different measures are used to compare two registered sclera images. These measures provide a quantitative score that describes the degree of similarity or conversely the degree of error/distortion between two images. To generate genuine scores, the measure is computed between pairs of images pertaining to the same subject; to generate impostor scores, the measure is computed between the first image (from the set of 
Chapter 2 Methods for sclera patterns matching using high resolution multispectral images

eight sequence images) of each subject pair. Having two registered images $I_{1}$ and $I_{2}$, and the two sclera masks, mask $_{1}$ and mask $_{2}$, we assess the similarity of the two images over the region mask $_{1} \bigcap$ mask $_{2}$ using different quantitative measures: root mean square error (RMSE) [41], cross-correlation (CORR), mutual information (MI) [42], normalized mutual information (NMI) [43], ratio-image uniformity (RIU) [42], and structural similarity index measure (SSIM) [44].

\subsection{Results}

Results are displayed using Receiver Operator Charateristic (ROC) curves and normalized score histograms. The results indicate lower $E E R$ values for left-eye-lookingleft and right-eye-looking-right compared to left-eye-looking-right and right-eye-lookingleft. This is due to the curvature of the eyeball and to the fact that facial features (such as the nose) partially obstruct the light directed to the left eye when looking right and the right eye when looking left.

\subsubsection{SURF}

The number of corresponding interest point pairs between images of the same eye will generate a genuine score and the number of corresponding interest point pairs between images of different eyes will generate an impostor score. The ROC and the normalized score distribution for both eyes, left and right gaze direction were obtained and are displayed in Appendix A. The approximate EER values are as follows: $0.37 \%$ for left-eye-looking left, $1.7 \%$ for left-eye-looking-right, $1.25 \%$ for right-eye-lookingleft and $0.75 \%$ for right-eye-looking-right as shown in the Table 2.4. The ROC and the distribution of scores are displayed in Appendix A. Results indicate that SURF method distinguishes very well between genuine and impostors. 


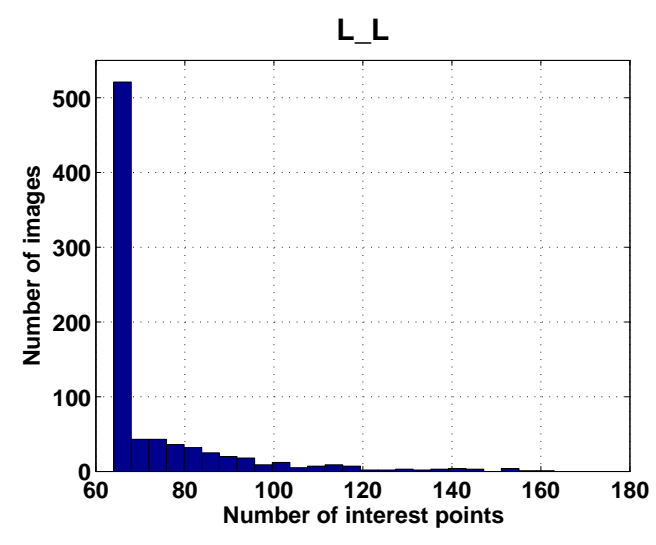

(a)

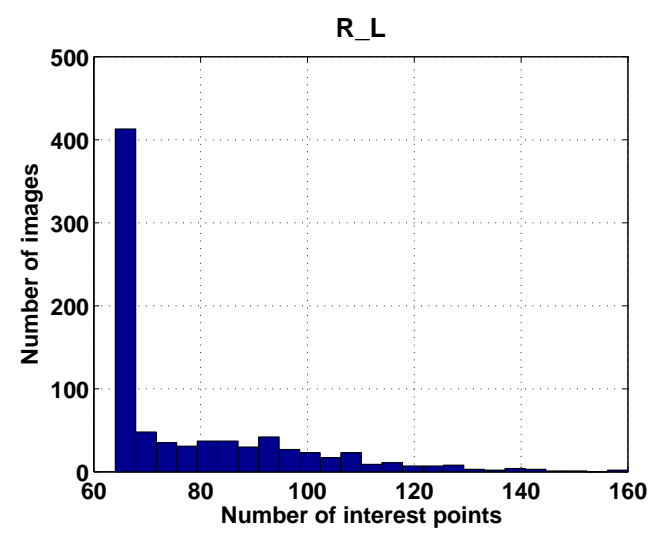

(c)

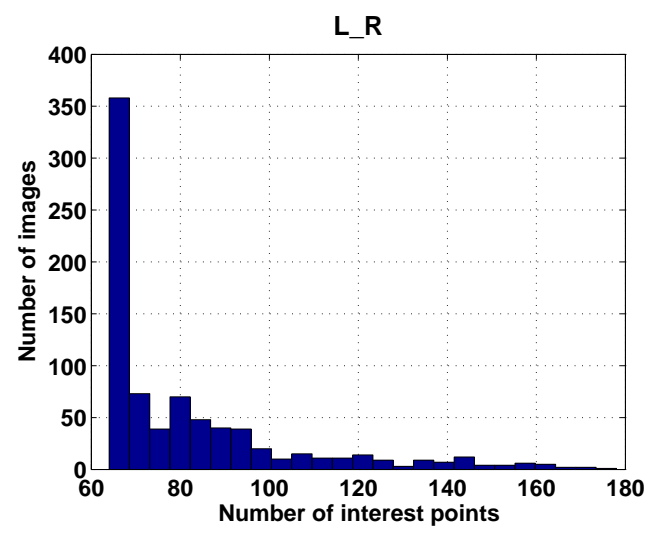

(b)

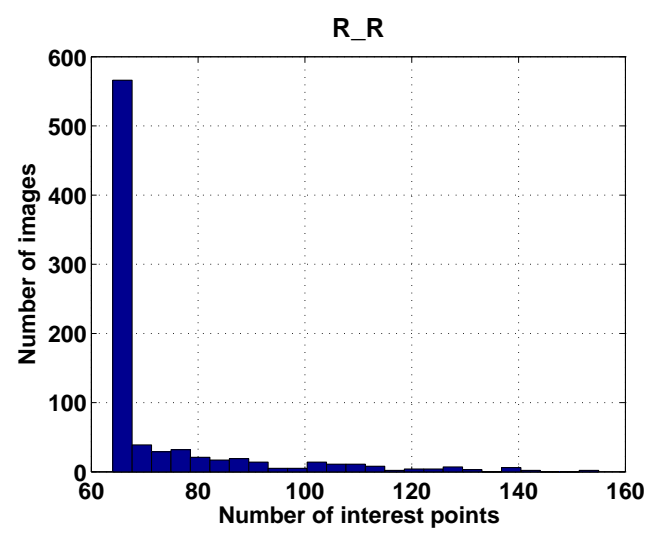

(d)

Figure 2.25 The histogram (25 bins) of the detected number of interest points for images of the eye (data collection 1). (a) Left-eye-looking-left (L_L). (b) Left-eye-looking-right (L_R). (c) Right-eye-looking-right ( $\mathrm{R} \_$). (d) Right-eye-looking-right (R_R) 
Chapter 2 Methods for sclera patterns matching using high resolution multispectral images

Table 2.4 The EER (\%) results when using SURF.

\begin{tabular}{||c||c|c|c|c||}
\hline \hline Performance measure & L_L & L_R & R_L & R_R \\
\hline \hline SURF & $0.37 \%$ & $1.7 \%$ & $1.25 \%$ & $0.75 \%$ \\
\hline \hline
\end{tabular}

Table 2.5 The average number of detected interest points for data collection 1

\begin{tabular}{||c||c|c|c|c||}
\hline \hline SURF & L_L & L_R & R_L & R_R \\
\hline \hline Average nr. of interest points & 73 & 81 & 78 & 72 \\
\hline \hline
\end{tabular}

\subsubsection{Minutiae points}

The ROC and the normalized score distribution for both eyes, left and right gaze direction were obtained. They are displayed in Appendix A. An approximate EER value of $9.5 \%$ is obtained for left-eye-looking-left, $10.3 \%$ for left-eye-looking-right, $12 \%$ for right-eye-looking-left, and $11.5 \%$ for right-eye-looking-right as shown in Table 2.6. A better segmentation of the conjunctival vasculature, a better detection of the finer blood vessels, and also a more accurate localization of the centerline of the blood vessels, may improve the value of EER when minutiae points are used for matching.

\subsubsection{Direct correlation}

The ROC and normalized score plots were obtained for all the measures mentioned in Section 2.7.3, for both eyes, and both gaze directions and are displayed in Appendix A. The approximate values of EER are displayed in Table 2.7 for left-eye-looking-

Table 2.6 The EER (\%) results when using minutiae points.

\begin{tabular}{||c||c|c|c|c||}
\hline \hline Performance measure & L_L & L_R & R_L & R_R \\
\hline \hline Minutiae points & $9.5 \%$ & $10.3 \%$ & $12 \%$ & $11.5 \%$ \\
\hline \hline
\end{tabular}


Chapter 2 Methods for sclera patterns matching using high resolution multispectral images

Table 2.7 The EER (\%) results when using different correlation methods.

\begin{tabular}{|c|c|c|c|c|}
\hline Performance measure & $\mathbf{L}_{-} \mathbf{L}$ & $\mathbf{L} \_\mathbf{R}$ & R_L & $\mathbf{R} \_\mathbf{R}$ \\
\hline RMSE & $1.51 \%$ & $4.75 \%$ & $5.6 \%$ & $2 \%$ \\
\hline CORR & $0.1 \%$ & $3.5 \%$ & $4.75 \%$ & $1.25 \%$ \\
\hline MI & $2 \%$ & $5 \%$ & $6.4 \%$ & $2.57 \%$ \\
\hline NMI & $0.7 \%$ & $3.4 \%$ & $5.2 \%$ & $1.6 \%$ \\
\hline RIU & $4.6 \%$ & $6.25 \%$ & $7.5 \%$ & $5.1 \%$ \\
\hline SSIM & $1.25 \%$ & $4.3 \%$ & $6 \%$ & $4 \%$ \\
\hline
\end{tabular}

left (L_L), left-eye-looking-right (L_R), right-eye-looking-left (R_L), right-eye-lookingright ( $\left.R \_R\right)$. The best performance is obtained when using the correlation measure, followed by the normalized mutual information scheme. If the eye images with the exposed sclera region that are to be compared are taken from approximately the same viewing angle, then direct correlation measures may be used as a matching method.

\subsection{Score-level Fusion}

In this work, min-max technique is used to normalize and fuse the minutiae scores with that of the direct correlation methods. Correlation, mutual information, normalized mutual information, and structural similarity index are similarity measures. Root mean square error, and ratio intensity uniformity are dissimilarity measures. A dissimilarity score is transformed into a similarity score by subtracting the normalized score from 1. Minutiae scores contain information about the veins only, while the direct correlation methods characterize the entire sclera surface. The results for score level fusion using the sum rule, max rule and min rule [45] shown in Tables 2.8 , indicate that for the fusion of minutiae scores with CORR, RIU, SSIM, RMSE 
Chapter 2 Methods for sclera patterns matching using high resolution multispectral images

scores, sum rule and the max rule perform the best. For the fusion of minutiae scores with MI scores, the sum and min rules have the best results, and for the fusion of minutiae scores with NMI scores, the min rule is the best method. The ROC and the distribution of scores are displayed in Appendix A.

\subsection{Summary}

The work presented in this chapter investigates the feasibility of using multispectral conjunctival vasculature in an ocular biometric system. To complement the loss of information from non-frontal iris images, additional details such as the sclera surface and its blood vessels are exploited for recognition. Iris patterns are better discerned in the NIR spectrum while vasculature patterns are better observed in the visible spectrum (RGB). Therefore, using multispectral images of the eye ensures that both, the iris and the sclera, are successfully imaged. In this chapter the spectral bands of multispectral imaging are presented and the color infrared images are described. In order to initiate the research for sclera texture and the accompanying blood vessels seen on its surface, a multispectral database of eye images, composed of two collections is assembled. The first collection consists of eight sequential eye images/eye/gaze direction collected from 103 subjects, with the camera being focused on the sclera region. The second collection consists of four images of the eye/eye/gaze direction collected from 31 subjects, the camera being focused on the iris region. The four images/eye/gaze direction are collected after the subject alternates the gaze direction from left or right to frontal and then back to left or right, so that the intra-class variation is higher. Each component of the color infrared image (NIR, red, green and blue) are individually subjected to denoising pre-processing method based on wavelet transform. A novel sclera segmentation method based on normalized sclera index fol- 
Chapter 2 Methods for sclera patterns matching using high resolution multispectral images

Table 2.8 The EER (\%) results of the fusion of minutiae scores with different correlation measures.

\begin{tabular}{||c||c|c||c|c||c|c||}
\hline \hline \multirow{2}{*}{} & \multicolumn{2}{|c|}{ CORR } & \multicolumn{2}{c||}{ MI } & \multicolumn{2}{c||}{ NMI } \\
\cline { 2 - 7 } & L_L & L_R & L_L & L_R & L_L & L_R \\
\hline Sum rule & $0.3 \%$ & $3 \%$ & $3.5 \%$ & $6.1 \%$ & $4.4 \%$ & $6.2 \%$ \\
\hline Max rule & $0.125 \%$ & $3 \%$ & $9 \%$ & $9.2 \%$ & $9.6 \%$ & $10 \%$ \\
\hline Min rule & $9 \%$ & $9.65 \%$ & $2 \%$ & $6.25 \%$ & $0.525 \%$ & $3.9 \%$ \\
\hline \hline \multirow{2}{*}{ Sum rule } & $4.25 \%$ & $1.25 \%$ & $7.2 \%$ & $4.2 \%$ & $7.95 \%$ & $6 \%$ \\
\hline Max rule & $4.75 \%$ & $1.25 \%$ & $9.75 \%$ & $7.95 \%$ & $11.75 \%$ & $11.5 \%$ \\
\hline Min rule & $11 \%$ & $11 \%$ & $7.5 \%$ & $4.1 \%$ & $5.9 \%$ & $1.75 \%$ \\
\hline \hline \multirow{2}{*}{ R_L } & R_R & R_L & R_R & R_L & R_R \\
\cline { 2 - 7 } & L_L & L_R & L_L & L_R & L_L & L_R \\
\hline Sum rule & $3.1 \%$ & $5.1 \%$ & $1.5 \%$ & $3.75 \%$ & $1.65 \%$ & $3.95 \%$ \\
\hline Max rule & $4.6 \%$ & $6.25 \%$ & $1.5 \%$ & $4.75 \%$ & $2.8 \%$ & $4 \%$ \\
\hline Min rule & $9.6 \%$ & $10 \%$ & $9.25 \%$ & $10.1 \%$ & $3.5 \%$ & $6.25 \%$ \\
\hline \hline Sum rule & $7.25 \%$ & $5.55 \%$ & $5 \%$ & $2.95 \%$ & $5.9 \%$ & $3.25 \%$ \\
\hline Max rule & $7.75 \%$ & $5.1 \%$ & $5.5 \%$ & $2 \%$ & $6.3 \%$ & $4.5 \%$ \\
\hline Min rule & $11.9 \%$ & $11.6 \%$ & $12 \%$ & $11.6 \%$ & $7.9 \%$ & $5.75 \%$ \\
\hline \hline
\end{tabular}


Chapter 2 Methods for sclera patterns matching using high resolution multispectral images

lowed by thresholding is applied to the first collection of the multispectral database. The pupil region segmentation based on power law transformation and multiple fitted ellipses is described. Processing techniques such as vasculature enhancement using a selective enhancement filter for lines, and implicitly for blood vessels is presented. Further, the images of the eye are registered with a global smooth and a local affine transformation based on intensity values. The design of three different feature extraction and matching methods is presented. The first one is based on interest-point detection and utilizes the entire sclera region including the vasculature pattern; the second is based on minutiae points on the vasculature structure; and the third is based on direct correlation. While the first two techniques do not need an explicit image registration scheme, the third technique relies on image registration. The results demonstrate the validity of using the sclera surface and the conjunctival vasculature for recognition and support further investigation in this area of research. 


\section{Chapter 3}

\section{Fusion of iris patterns with scleral}

\section{patterns}

Iris recognition performance is greatly and negatively influenced by the occlusions, the lighting conditions and the direction of the gaze of the eye with respect to the acquisition device. The use of the sclera as a biometric may be significant in the context of iris recognition, when changes in the gaze angle of the eye can result in non-frontal iris images that cannot be easily recognized. The more the gaze direction deviates from the frontal pose, the more information from the iris texture is lost and the more information from the sclera region is gained. The combined sclera and the iris texture may be used in non-cooperative recognition when the probability of nonideal iris occurrence is greatly increased. By utilizing the texture of the sclera along with the vascular patterns evident on it, the performance of an iris recognition system can potentially be improved. The block diagram of the proposed system is shown in Figure 3.1. In this approach the multispectral collection 1 is used. Image acquisition and image denoising were presented in Chapter 2. 


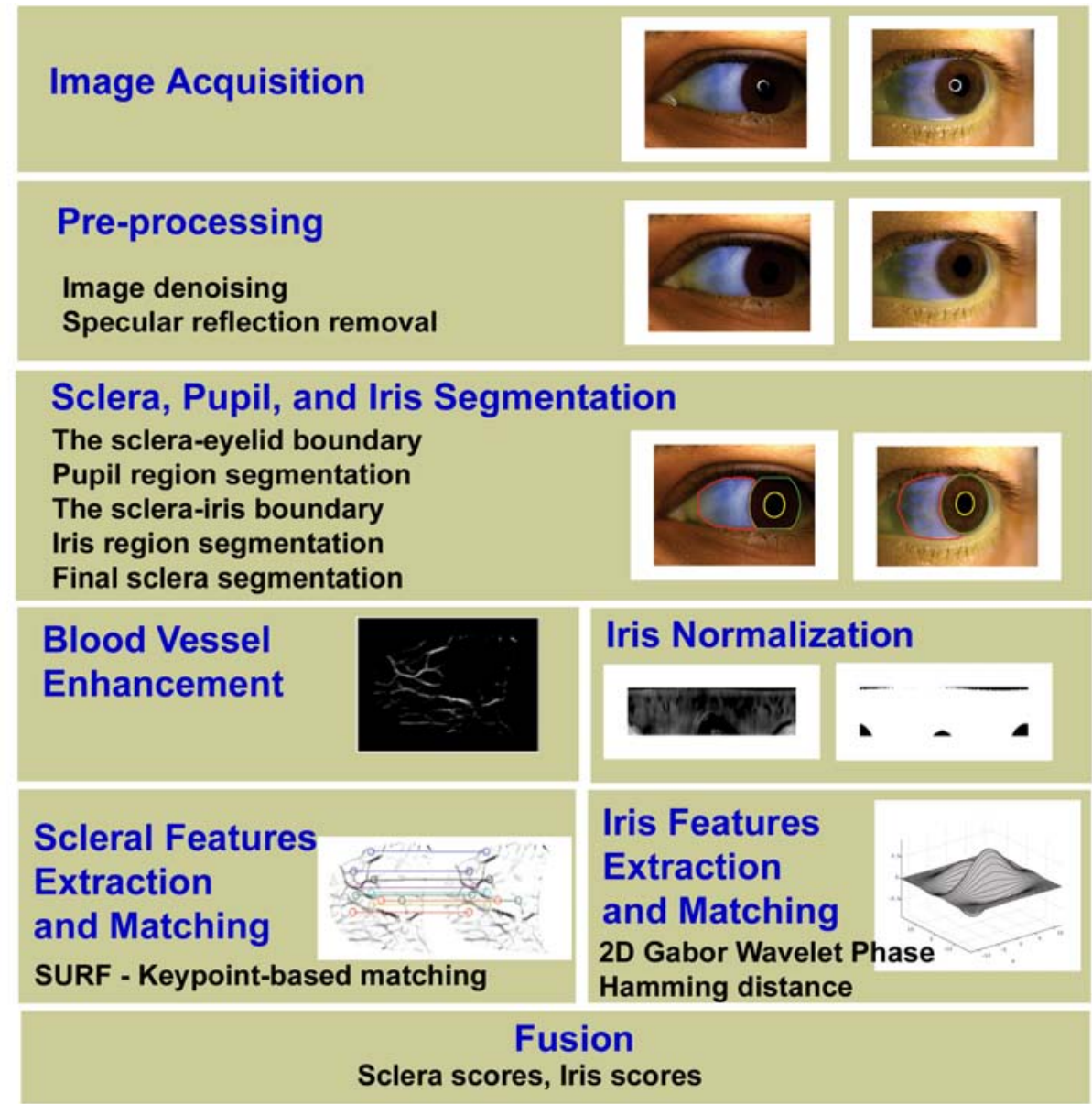

Figure 3.1 Block Diagram 


\subsection{Specular reflection detection and removal}

The specular reflection (the ring-like shape and the highlights due to the moisture of the eye) is detected in a two step algorithm and removed by a fast inpainting procedure. In some images, the ring-like shape may be an incomplete circle, ellipse, or an arbitrary curved shape with a wide range of intensity values. It may be located partially in the iris region, its precise detection and removal being more important especially since the iris texture has to be preserved as much as possible. In the first step of the algorithm, a good detection of the ring-like shape specular reflection is accomplished by converting the RGB image into $\mathrm{L}^{*} \mathrm{a}$ b color scheme followed by a range filtering through which every pixel in the image is replaced with the difference between the maximum and minimum value in a $3-b y-3$ neighborhood around the corresponding pixel. The ring-like shape specular reflection is obtain by applying a threshold of value $t h=30$ to the luminance component and it is removed by morphological dilation and inpainting. The high value of the threshold isolates the ring-like shape specular reflection from the other highlights. The remaining specular reflections are detected using different intensity threshold values for each component: 0.8 for NIR, 0.7 for red and 0.8 for green. Only regions with less then 1000 pixels in size are labeled as specular reflection, are morphologically dilated and inpainted. In digital inpainting, the information from the boundary of the region to be inpainted is propagated smoothly inside the region. The value to be inpainted at a pixel is calculated using a PDE equation ${ }^{1}$ in which partial derivatives are replaced by finite differences between the pixel and its eight neighbors. The final specular reflection mask consists of the logical $O R$ operation between the masks obtained in two steps.

\footnotetext{
${ }^{1}$ http://www.mathworks.com/matlabcentral/fileexchange/4551
} 


\subsection{Ocular Region Segmentation}

The color image segmentation is a challenging process. It is influenced by illumination, and the image texture as a result of the saturation, hue and blending of the colors. Since the scope of the algorithm is to fuse the information provided from the iris and the sclera, an accurate labeling of pixels pertaining to both regions is very important. Uneven illumination, the wide variety of intensity values across the eye surface caused by the curved shape of the eyeball, reflections of the light on the skin surface and occasionally the presence of plenty of mascara, will make the segmentation of the iris and the sclera regions a difficult process and even more problematic to achieve in nonfrontal images of the eye. Exposed on the sclera surface, the conjunctival vasculature appears as dark curved lines, of different thickness that intersect in a random way. Through the segmentation process they will be distinguished from wrinkles, crows feet, and eyelashes. It is also important to have an accurate sclera contour along the eyelids, since the blood vessels may be located on the margins of the sclera. Regardless of the color of the iris, the algorithm to segment the sclera, the iris and the pupil has five steps as described below.

1. The sclera-eyelid boundary

2. Pupil region segmentation

3. The sclera-iris boundary

4. Iris region segmentation

5. Final sclera region segmentation 


\subsubsection{The Sclera-Eyelid Boundary}

The algorithm to segment the sclera region presented in Chapter 2 and used in [46] uses the threshold 0.1 to delineate the pixels pertaining to the sclera region and the background. The threshold value depends on the illumination of the eye and doesn't represent the optimal choice for all the images of the eye. Therefore an improved automatic segmentation of the sclera region free of thresholds is necessary. The method employed to segment the sclera region along the eyelid contour is inspired by the work done in the processing of LandSat imagery (Land + Satellite) [31]. A set of indices, are used to segment the vegetation regions in aerial multispectral images based on a different absorbtion of the NIR wavelength by regions with different saturation in water content. Similarly, the index that it's used for coarse sclera segmentation is based on the fact that the skin has lesser water content than the sclera, and hence exhibits a higher reflectance in NIR. Since water absorbs NIR light, the corresponding regions appear dark in the image and, hence, the sclera appears darker in the image. The algorithm is as follows:

1. Geometrically resize the near-infrared and green component by a factor of $1 / 2$.

2. Compute an index called the normalized sclera index $N S I(x, y)=\frac{N I R(x, y)-G(x, y)}{N I R(x, y)+G(x, y)}$, where $N I R(x, y)$ and $G(x, y)$ are the pixel intensities of the NIR and green components, respectively, at pixel location $(x, y)$. The difference NIR(x,y)$G(x, y)$ is larger for pixels pertaining to the sclera region; it is then normalized to help compensate for the uneven illumination. Figure 3.2 (b) displays the normalized sclera index for all three categories as specified by the Martin-Schultz scale: light colored iris, dark colored iris and mix colored iris.

5. Using the integral image as explained in [36], for each pixel of $(x, y)$ of $N S I$ compute the mean $\mu$ and the standard deviation $\sigma$ for a neighborhood of radii 

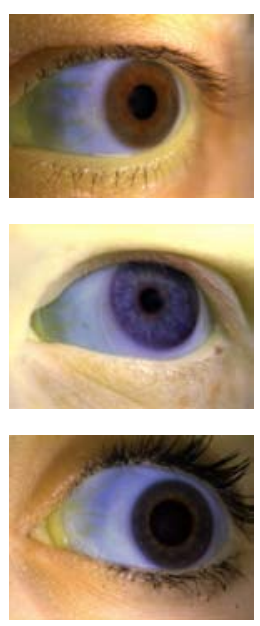

(a)
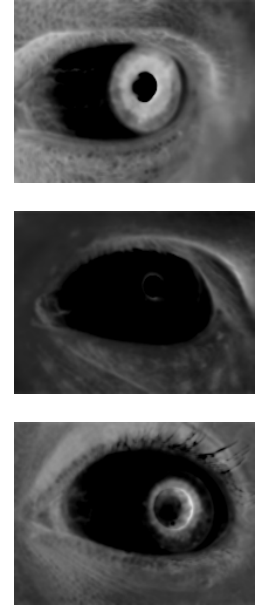

(b)
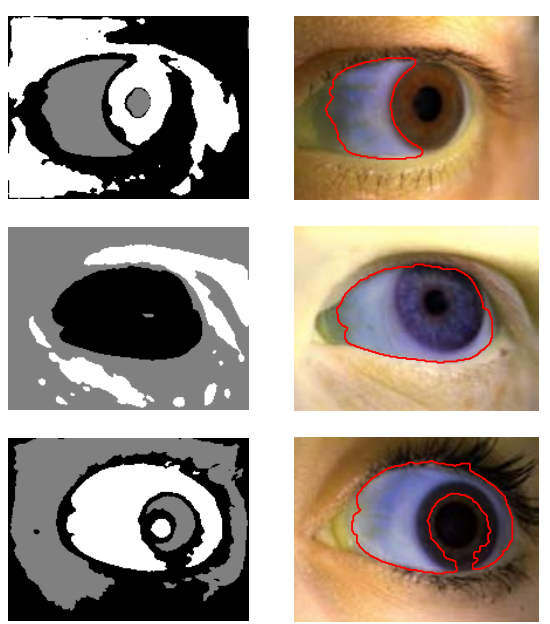

(c)

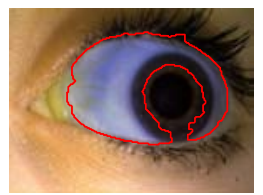

(d)

Figure 3.2 The sclera-eyelid boundary. The first row displays the results for dark colored iris, the second row displays the results for light colored iris, and the third row displays the results for mix colored iris: (a) original composite image. (b) The normalized sclera index (NSI). (c) The output of the K-means clustering algorithm. (d) Sclera-eyelid boundary imposed on original composite image.

\section{$0,1,3,5$ and 7 pixels.}

6. Build the feature vector Features $=[\mu 0, \mu 1, \mu 3, \mu 5, \mu 7, \sigma 0, \sigma 1, \sigma 3, \sigma 5, \sigma 7]$, where $\mu$ is the mean and $\sigma$ is the standard deviation, column vectors for the above mentioned radii.

7. Three clusters are considered: the sclera, the iris and the background. Apply k-means clustering algorithm to the feature vector Features with $k=3$, where $k$ is the number of clusters. The algorithm will partition all the pixels in three clusters and will return the cluster centroid locations. As seen in Figure 3.2 (c), for all three categories as specified by the Martin-Schultz scale (light colored iris, dark colored iris and mix colored iris) the sclera-eyelid boundary is very well defined. 
8. For each cluster compute the mean value of all the pixels from NSI image. The cluster with the lowest mean represents the sclera region. For all three categories as specified by the Martin-Schultz scale (light colored iris, dark colored iris and mix colored iris), the sclera cluster exhibits a good segmentation along the sclera-eyelid boundary, but differs in regard of sclera-iris boundary. For dark irides (brown and dark brown), the sclera region excluding the iris is localized (Figure 3.2(d) the first row, referred henceforth as $I_{S}$ ). Thus, in this case, the sclera-iris boundary is detected, and further segmentation of the sclera and iris is not required. For light irides (blue, green, etc.), regions pertaining to both the sclera and iris are segmented (Figure 3.2 (d) the second row, referred henceforth as $\left.I_{S}\right)$. Here, further separation of the sclera and iris is needed. For mixed irides (blue or green with brown around pupil), the region of the sclera and the light colored portion of the iris are segmented as one region (referred henceforth as $\left.I_{S}\right)$. The dark portion of the iris (brown) is not included (Figure 3.2(d) the third row). Here, further separation of the sclera and the portion of the iris is needed. To find the boundary between the sclera and the iris regardless of the color of the iris, the pupil is detected. The convex hull of the segmented region $I_{S}$ and the pupil region will contain the sclera, the pupil, and the iris or the portion of the iris. This region is referred to as $I_{S I P}$ and is further processed. Since the proposed algorithm does not deduce the color of the iris, it is applicable to all images irrespective of the eye color. As seen in Figures 3.2 (c), the location of the pupil is also visible as part of the sclera cluster in dark and mixed irides, or as a lighter disk within the sclera region in light irides. This information can be exploited only if the color of the iris is known in advance. Therefore, in Section 3.2.2 we present an automatic way of finding the pupil location regardless of the color of the iris. 


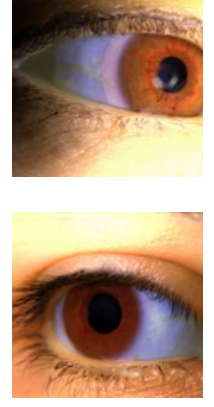

(a)
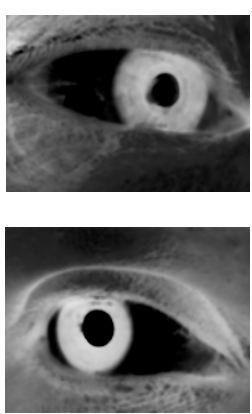

(b)
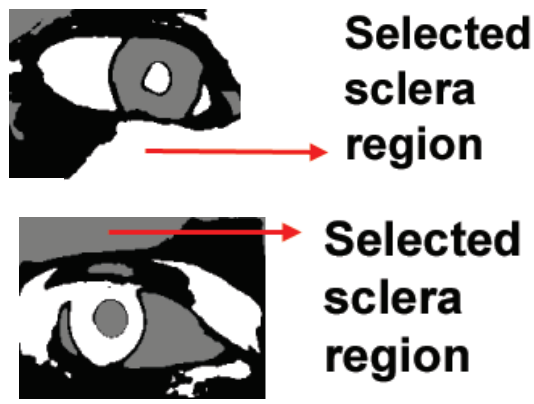

Selected

sclera region

Figure 3.3 The sclera-eyelid boundary errors. First row represents the errors in images with strong uneven illumination. Second row represents the errors in images with large specular reflections on the skin. (a) Original composite image. (b) Normalized sclera index. (c) The output of the k-means algorithm.

9. Sclera mask is represented by the largest connected region from the sclera cluster.

10. Geometrically resize the mask of the sclera to the original size of the near-infrared or green component.

The algorithm fails to segment properly the sclera region for images with strong uneven illumination or with large areas of specular reflections on the skin surface. Such areas may represent the largest connected region from the sclera cluster and may be erroneously selected as sclera region (Figure 3.3).

For a total of 36 images, from 6 subjects the sclera region is segmented manually.

\subsubsection{Pupil Region Segmentation}

The location of the pupil is needed to build $I_{S I P}$ and further find the boundary between the sclera and the iris regardless of the color of the eye. It is also needed for iris segmentation presented in Section 3.2.4. The algorithm implemented to segment the pupil region is based on different thresholds applied to the $N I R$ component to detect the pixels with low intensity values. This will result in the detection not only 

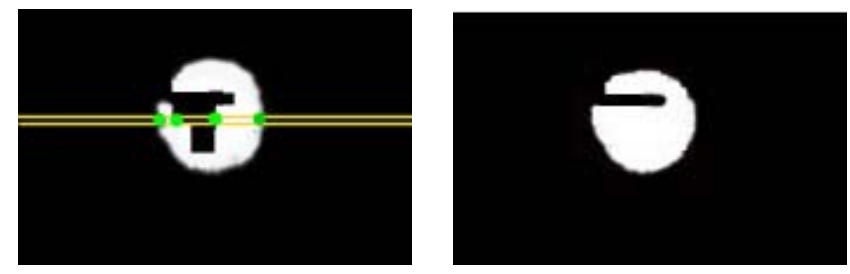

Figure 3.4 Pupil region segmentation. Filling the holes at the iris-pupil boundary due to inpainting of the specular reflection that results in higher pixel value than the pupil pixel value.

of the pupil region but also of the eyelashes or other darker regions in the image. To discriminate among these pixels the round shape of the pupil is exploited. The algorithm is as follows:

1. Find the Otsu threshold for NIR image, otsuTh.

2. Through an iterative process when $n$ varies from $\max (\min (\mathrm{NIR}(\mathrm{x}, \mathrm{y})), 0)$ to 0.3 with the increase step of 0.02 , compute the threshold $T h=n \times$ otsuTh

3. For each $T h$ find the pixels $N I R(x, y)<T h$. Consider the connected regions with more than 400 pixels (Figure 3.5).

4. For each connected region fill the possible holes caused by improper inpainting of the specular reflection along the iris-pupil boundary as described in [47]. If the binary mask of the pupil doesn't contain a hole, then along an horizontal line there will be only one crossing from 0 to 1 , otherwise it will have more crossings. Along each horizontal line all detected points that belong to a hole are filled as depicted in Figure 3.4.

5. For each connected region compute the metric $M=\frac{4 \times p i \times a r e a}{\text { perimeter }}{ }^{2}$. The closer the shape of the connected region to a circle, the closer to 1 the value of the metric. In non-frontal iris images, the pupil region is approximated with an ellipse. 
Since the pupil is not a very elongated ellipse, the metric $M$ will have a value close to 1 .

6. Choose the connected region with the highest value of the metric $M$. This represents the contour of the pupil region. Find its contour and fit an ellipse $E_{\text {pupil }}\left(x_{p}, y_{p}, M_{p}, m_{p}, \theta_{p}\right)$ where $\left(x_{p}, y_{p}\right)$ represents the center coordinates of the ellipse, $M_{p}, m_{p}$ represents the major and minor axes and $\theta_{p}$ represents the tilt of the ellipse.

Since the value of the metric $M$ is based on the area and perimeter values, the algorithm fails to properly segment the pupil region if by inpainting a large specular reflection area located at the boundary between the pupil and the iris, changes drastically the elliptical contour of the pupil. In such cases the failure to segment properly the pupil region is accentuated by heavy mascara on the eyelashes. To solve this problem, constraints on pupil location may be added to the algorithm to improve the segmentation performance such as considering only half the image, according to the gaze direction or locate the pupil region in the ellipse mask fitted to the sclera region as described in Chapter 2 and used in [48] (The contour of the convex hull of the sclera region is detected. Using Hough transform for line detection, the highest peak corresponding to the longest line is selected and removed. An ellipse is fitted to the remaining contour pixels. The search for the pupil region is constraint among the pixels located within the fitted ellipse).

Example of images with segmented pupil region are displayed in Figure 3.6

For a total of 19 images pertaining to 6 subjects, the pupil is segmented automatically. 

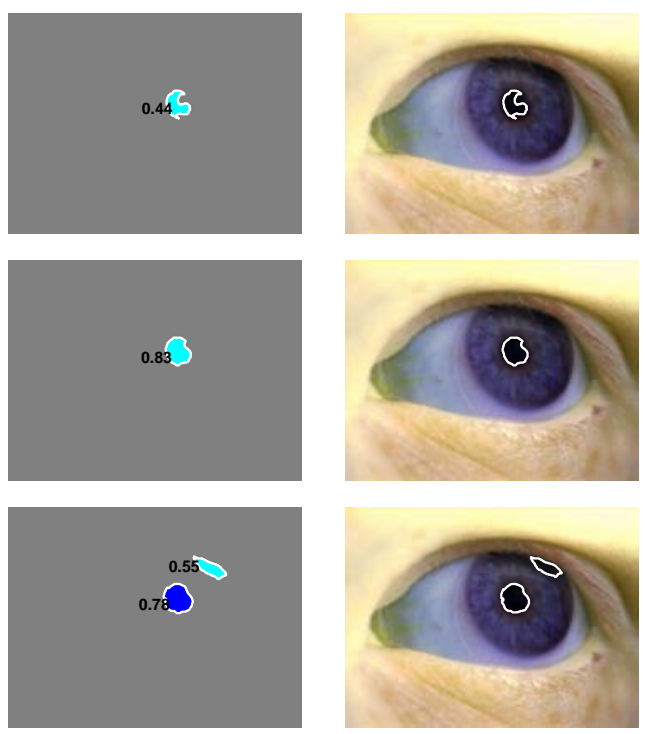

(a)

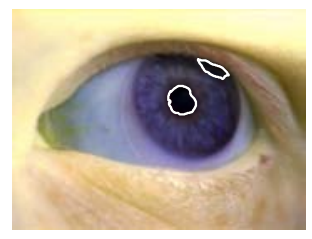

(b)
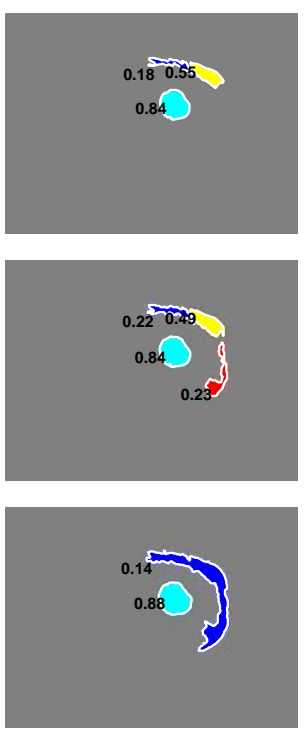

(c)
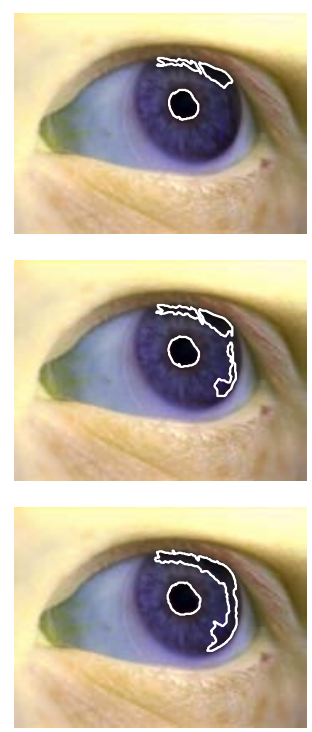

(d)

Figure 3.5 Pupil region segmentation. (a) The metric $M$ for the thresholds $0.04,0.1$, and 0.16 . (b) Thresholding result (the contour) imposed on the composite image, thresholds $0.04,0.1$, and 0.16 . (c) The metric $M$ for the thresholds $0.18,0.2$, and 0.24 . (d) Thresholding result (the contour) imposed on the composite image, for thresholds $0.18,0.2$, and 0.24 .
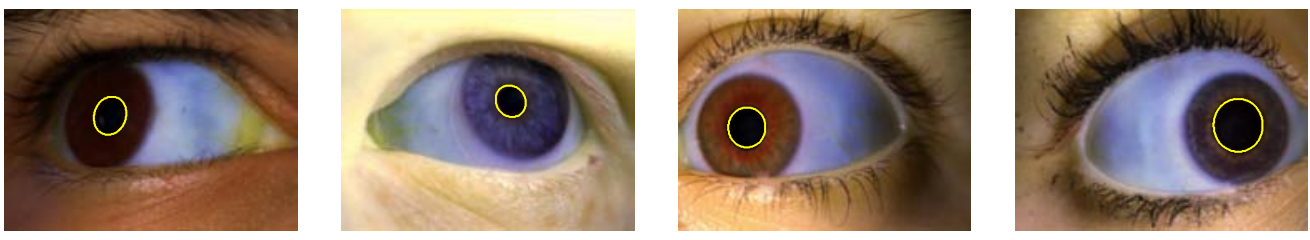

Figure 3.6 Pupil region segmentation. Examples. 

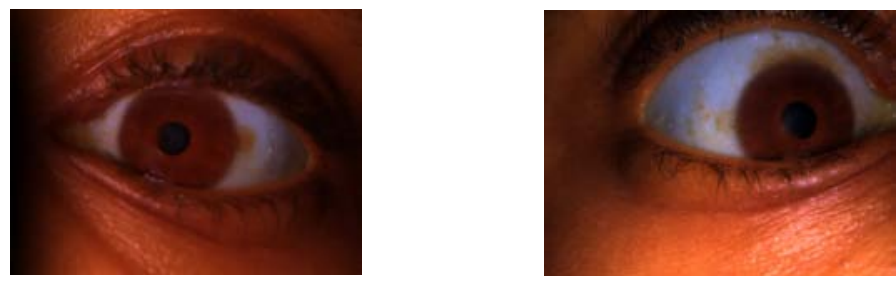

Figure 3.7 Ocular images with a greater amount of melanin around the iris region.

\subsubsection{The Sclera-Iris Boundary}

The convex hull of the segmented sclera region and the pupil region will contain the sclera, the pupil and the iris or the portion of the iris. To find the sclera-iris boundary we use the method defined in Chapter 2, subsection 2.4.3. This method fails to find the correct sclera-iris boundary for ocular images that reveal a greater amount of melanin at the limbus boundary, Figure 3.7.

\subsubsection{Iris Region Segmentation}

The segmentation of the iris region in non-frontal iris images is a challenging process. Existing algorithms use NIR images and aim to find the best fit contour along the limbus region. Previous research for multispectral iris is encountered in [49] where an automatic localization of the spatial extent of the iris structure is detected in two steps: a pupillary boundary detection followed by a limbic boundary detection. However this study was performed on frontal iris images. Besides the non-frontal position of the iris, another challenge encountered in the process of iris segmentation for our dataset was the improper illumination of the iris in some images. Our iris segmentation algorithm uses the color information (color gradient) provided by the composite (CIR) image, the elliptical parameters of the pupil and the sclera-iris boundary. The algorithm is as follows (subscript $i$ stands for iris and $p$ for pupil): 
1. Using the parameters of the ellipse that fit the contour of the pupil, unwrap the sclera mask. The angular resolution is ang_res $=360$ and the radial resolution is rad_res $=$ radius $/$ res where res $=120$ and radius is of variable size according to the dilation of the pupil. To detect the best radial resolution, through an iterative process and starting with the value radius $=3$, the radius is increased by 1 and sclera mask unwrapped for each iteration. The number of pixels pertaining to the unwrapped sclera mask along the lines is computed and the maximum value is detected. The iterative process stops when the location of the maximum value is greater then res -10 . This is based on the fact that as the sclera mask is unwrapped, the shape of the unwrapped sclera can be approximated with that of a trapezoid with the larger base up. The value of the resolution rad_res is very important for the ratios calculated in the next steps. The results are depicted in Figure 3.9 (b). The sclera region is represented by the white region. To better visualize the location of the iris, pupil, and sclera region in the unwrapped sclera mask and pupil mask, the composite images from Figure 3.2 (a), representing the three categories in the Martin-Schultz scale, are unwrapped and displayed in Figure 3.8.

2. Using the parameters of the ellipse that fit the contour of the pupil, elliptical unwrap the pupil mask with the determined angular and the radial resolutions. The results are depicted in Figure 3.9 (a). The pupil region is represented by the gray region. Since the pupil parameters are used to unwrap the pupil mask, the iris-pupil boundary is represented by a straight line. Find the pupil-iris boundary $B_{p i}$.

3. Since the pupil parameters are used to unwrap the sclera mask, the sclera-iris boundary is represented by a a curved line as displayed with yellow color in 
Figure 3.9 (b). Find two lines $B_{\text {simin }}$ (red color) and $B_{\text {simax }}$ (blue color) corresponding to the minimum and the maximum position of the sclera-iris boundary (the yellow line). Two ratios are calculated $R_{\min }$ and $R_{\max }$, in order to find the parameters of two ellipses, $E_{\min }$ and $E_{\max }$, used to approximate the contour of the iris region.

$$
\begin{aligned}
& R_{\text {min }}=\frac{B_{\text {simin }}}{B_{p i}}, \\
& R_{\text {max }}=\frac{B_{\text {simax }}}{B_{p i}}
\end{aligned}
$$

4. Keeping the tilt angle and the center coordinates of the pupil, based on the two ratios and the pupil's major and minor axis values, calculate the major and minor axis of two ellipses Ellipse $_{\min }$ and Ellipse $_{\max }$, as follows:

$$
\begin{array}{llr}
R_{p} & = & M_{p} / m_{p}, \\
m_{i-} \text { max } & = & R_{\max } \times m_{p}, \\
m_{i \_} \text {min } & = & R_{\min } \times m_{p}, \\
M_{i \_} \text {min } & =R_{p} \times m_{i \_} \text {min }, \\
M_{i \_} \text {max } & =R_{p} \times m_{i \_} \text {max }
\end{array}
$$

The resulting ellipses $E_{\min }\left(x_{i}, y_{i}, M_{i-} \min , m_{i-} \min , \theta_{i}\right)$ and $E_{\max }\left(x_{i}, y_{i}, M_{i-} \max , m_{i-} \max , \theta_{i}\right)$ , where $\left(x_{i}, y_{i}\right)=\left(x_{p}, y_{p}\right)$, and $\theta_{i}=\theta_{p}$, are depicted in Figure 3.10. As it can be observed, the sclera-iris boundary is in between the two ellipses. The remaining steps are considered for a more accurate ellipse fitting to the contour of the iris.

5. Build an elliptical mask comprised of all the pixels inside the $E_{\max }$.

6. Apply the color gradient algorithm to the composite image, Figure 3.11 (a) and threshold it with $T h=0.1$. Impose the elliptical mask on the resulting image. Results, $I m_{C G}$, are displayed in Figure 3.11 (b). 
7. Using the parameters that fit the contour of the pupil, unwrap the image $I m_{C G}$ obtained in the previous step.

8. Calculate the sum of all the pixels along the rows and find the maximum value in between the two lines $B_{\text {simin }}$ (red color) and $B_{\text {simax }}$ (blue color).

9. Recalculate the ratios from step 4 and find the major and minor axis for an ellipse that will best fit the contour of the iris. Build an elliptical mask comprised of all the pixels inside the ellipse.

10. Find the convex hull of the sclera cluster and the elliptical mask and apply the hole filling algorithm as used in Section 3.2.2.

11. Similar to the algorithm presented in Chapter 2, Section 2.4.3, apply K-means algorithm with $k=2$ (the sclera and iris clusters) to the pixels inside the convex hull. Two binary images, a mask for the sclera region and a mask for the iris region represent the output.

12. Pixels pertaining to the sclera region, mostly those along the upper eyelid contour, may be labeled as iris pixels. Therefore, from the center of the pupil, build a set of rays to the contour of the iris mask and consider always the first intersection of the ray with the iris contour. This will provide the final iris contour. Fit an ellipse to this contour.

13. Find the maximum and minimum values along the $\mathrm{x}$ coordinate of the sclera mask and limit the iris mask in between these to values.

Examples of images with segmented iris are displayed in Figure 3.13.

The algorithm returns error to segment the iris in 12 images. For these images, the iris is segmented manually. Approximately $85 \%$ of the times the generated ellipse will fit the boundary region of the iris for both eyes both gaze directions. 


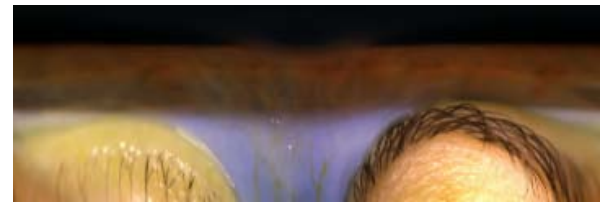

(a)

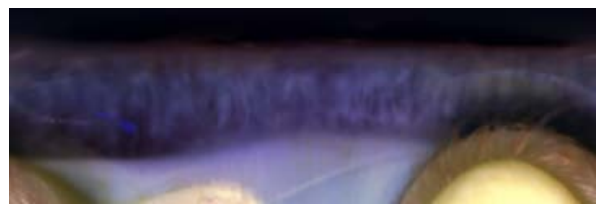

(b)

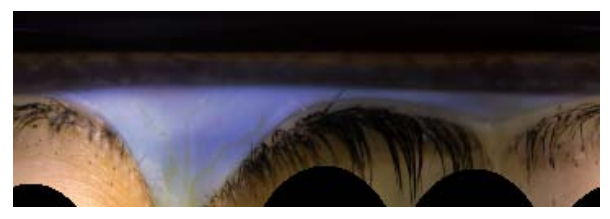

(c)

Figure 3.8 Iris segmentation. Elliptical unwrapping based on the pupil parameters: (a)Dark colored iris . (b) Light colored iris . (c) Mixed colored iris.
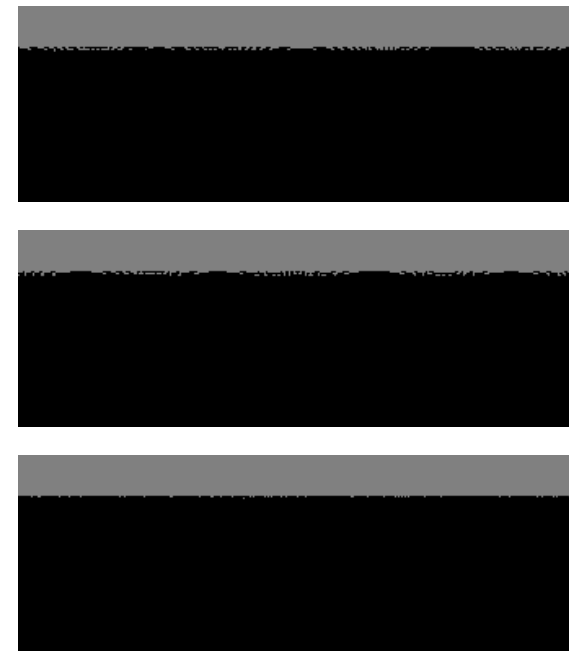

(a)
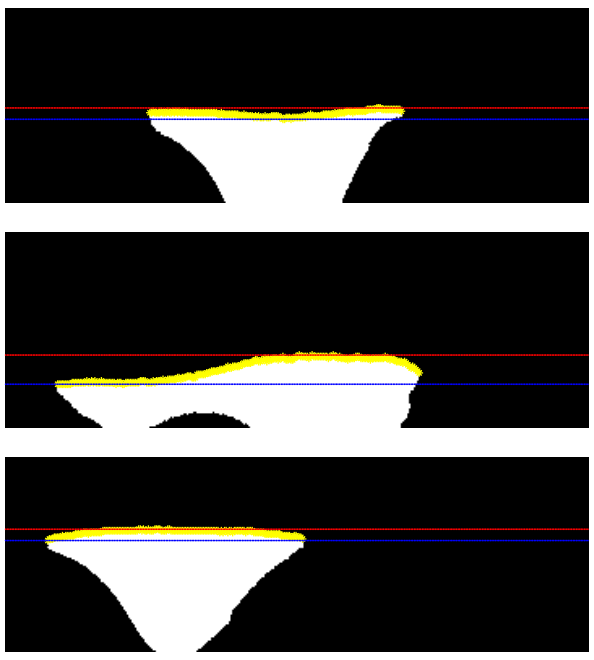

(b)

Figure 3.9 Iris segmentation. The first row displays the results for dark colored iris, the second row displays the results for light colored iris, and the third row displays the results for mix colored iris: (a) Pupil mask unwrapped. (b) Sclera mask unwrapped. 

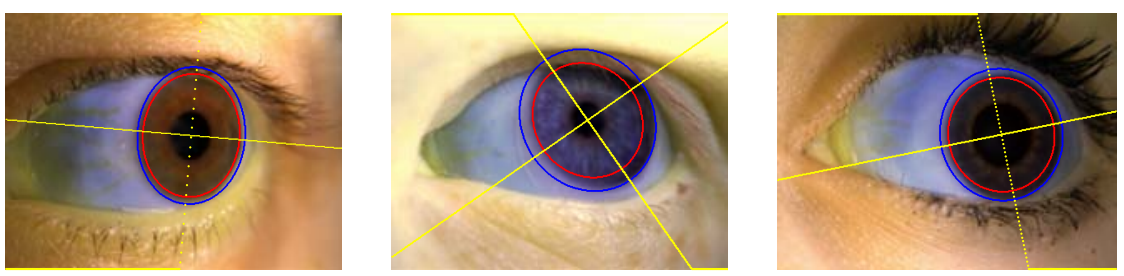

Figure 3.10 Iris segmentation. Contour of the two ellipses, Ellipse min $_{\text {and }}$ and Ellipse $_{\max }$, and their tilt imposed on the composite image.
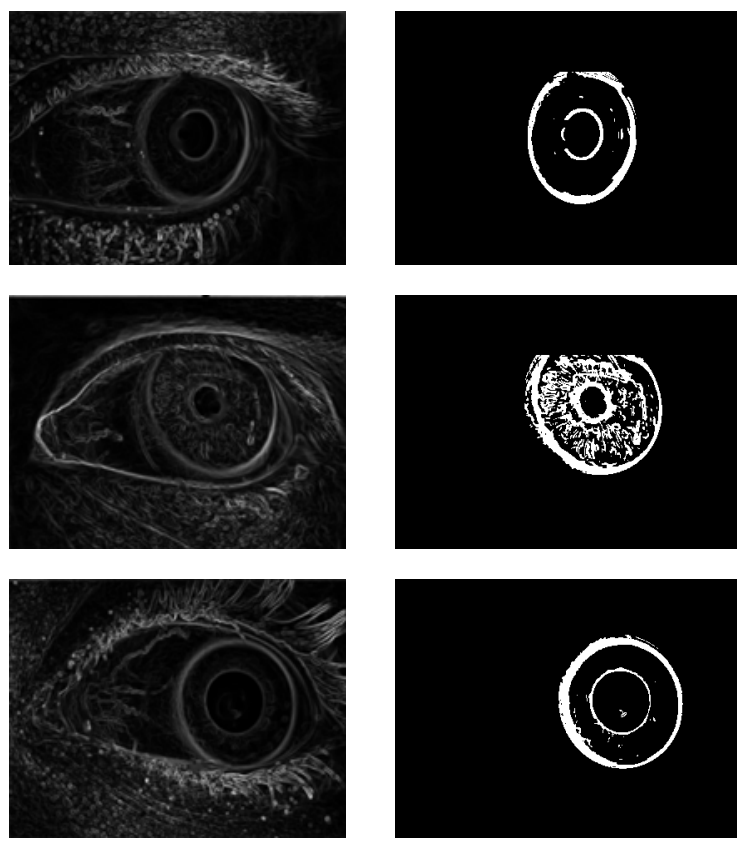

(a)

(b)

Figure 3.11 Iris segmentation: (a) Color gradient on composite image. (b) The threshold and Ellipse $_{\max }$ ellipse mask imposed on color gradient image ColorGrad $_{T h}$. 


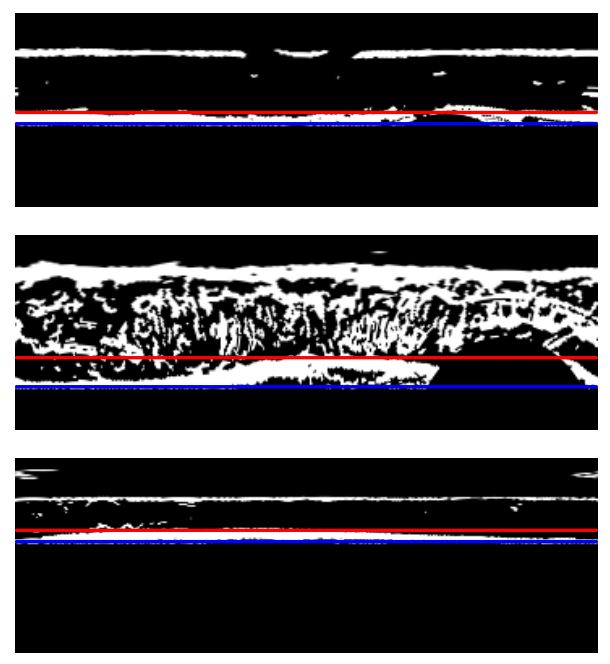

Figure 3.12 Iris segmentation. Image ColorGrad Th $_{\text {unwrapped along with }}$

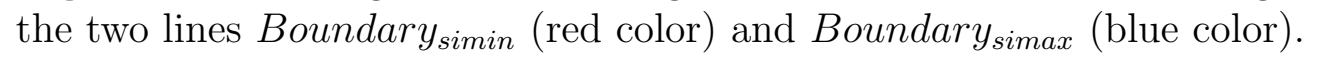

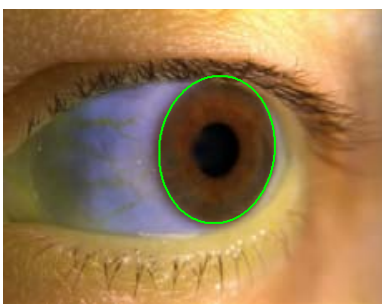

(a)

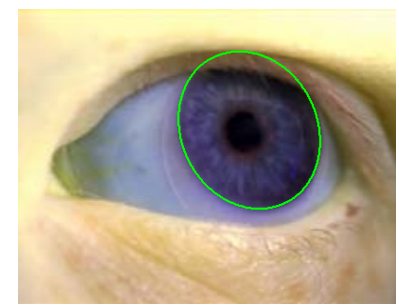

(b)

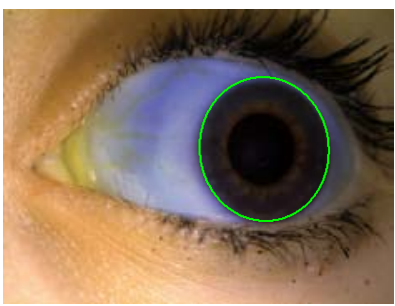

(c)

Figure 3.13 Examples of segmented irides: (a) Dark colored iris. (b) Light colored iris. (c) Mixed colored iris. 

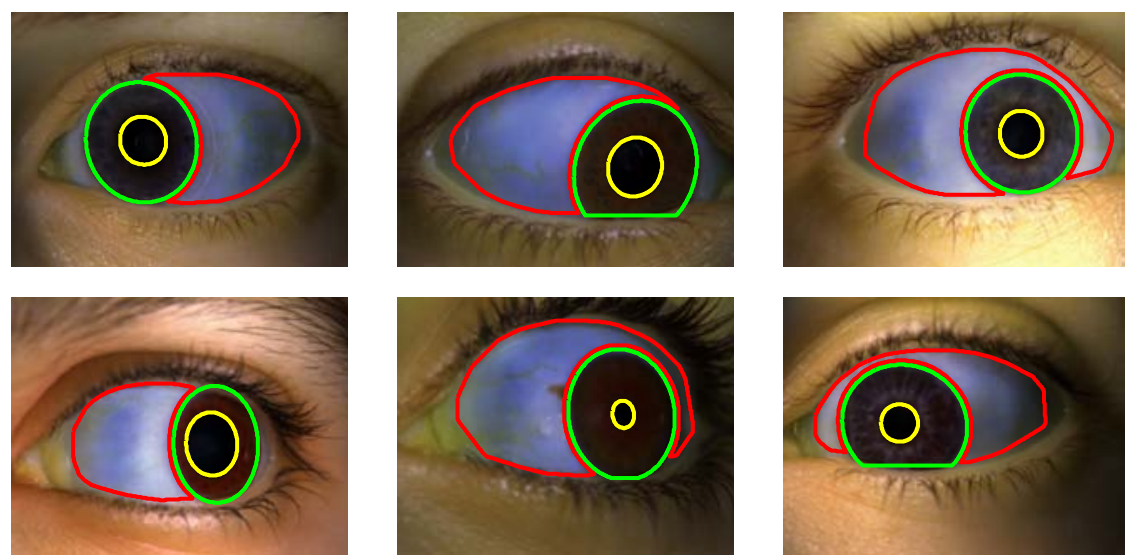

Figure 3.14 Examples of correct eye image segmentation.
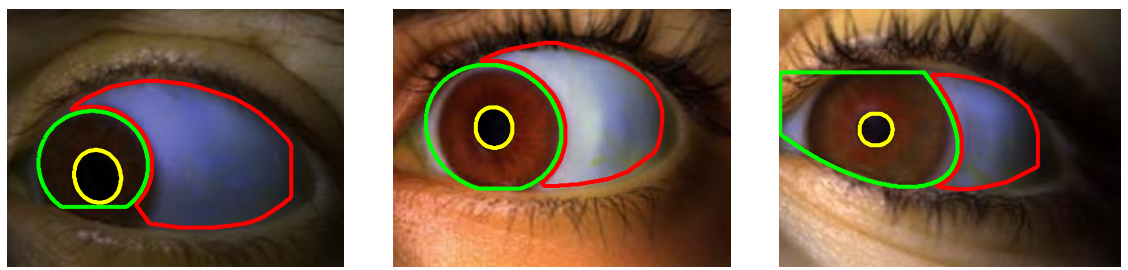

Figure 3.15 Examples of correct eye image segmentation.

\subsubsection{Final Sclera Region Segmentation}

The last step in the segmentation of the regions of interest for an image of the eye , is to finalize the segmentation of the sclera. This is accomplished as follows:

1. Build the convex hull of the sclera cluster obtained in Section 3.2.1.

2. Subtract the iris mask from the convex hull obtained in the previous step.

3. Erode the binary image with a structuring element of disk shape and size 5 . This will ensure that the contour line of the sclera is not included in the mask.

Example of correct eye image segmentation are illustrated in Figure 3.14.

Example of incorrect eye image segmentation are illustrated in Figure 3.15 


\subsection{Iris Feature Extraction}

Feature extraction is defined as the transformation of the input data into a set of features that capture the relevant information characteristic to the data. It consists of three steps: iris normalization, feature extraction using 2D Gabor wavelets and dissimilarity scores calculation using the Hamming distance measure.

\subsubsection{Iris Normalization}

The outcome of the iris segmentation process consists in iris regions of different sizes. The dimensional inconsistencies are mainly due to the pupil dilation, the viewing angle from which the image of the eye is captured, and the tilt of the head. For comparison purposes, the segmented irides are to be normalized and brought to the same size. An example code for elliptical unwrapping of the iris and pupil regions in non-ideal iris images that considers the center of the pupil as the center of the two ellipses, is used in [50] ${ }^{2}$. Using Daugman's rubber sheet model displayed in Figure 3.16, every iris pixel is mapped from the Cartesian coordinate system into the polar coordinate system $I_{(x, y)} \rightarrow I_{(r, \theta)}$ with an angular resolution of 360 and radial resolution of 64 , according to the equations:

$$
\begin{aligned}
& x_{(r, \theta)}=(1-r) \times x_{p}(\theta)+r \times x_{i}(\theta) \\
& y_{(r, \theta)}=(1-r) \times y_{p}(\theta)+r \times y_{i}(\theta)
\end{aligned}
$$

where $I_{(x, y)}$ is the iris image in Cartesian coordinates, $I_{(r, \theta)}$ is the iris image in polar coordinates, $\left(x_{p}, y_{p}\right)$ and $\left(x_{i}, y_{i}\right)$ are the coordinates of the boundary of the pupil and iris along the direction $\theta$. The radius $r$ varies in the interval $[0,1]$ and $\theta$ in the interval $[0,2 \pi]$. This model accounts for the pupil dilation, takes in consideration that the center locations of the pupil and iris are different, but is not invariant to

\footnotetext{
${ }^{2}$ http://www.csee.wvu.edu/ xinl/demo/nonideal_iris.html
} 

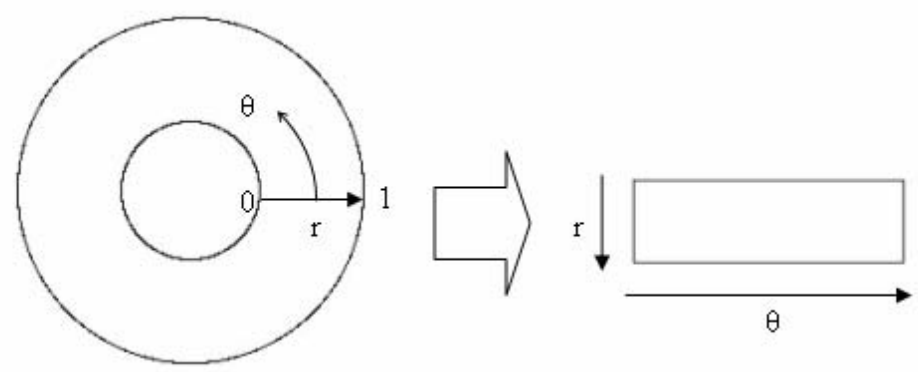

Figure 3.16 Iris normalization: Daugman rubber sheet model.

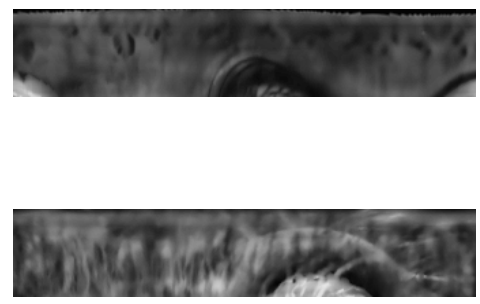

(a)
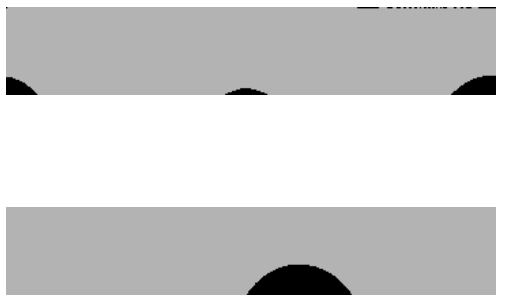

(b)

Figure 3.17 Examples for iris normalization: (a) Normalized iris. (b) The mask for occlusions.

rotations. Since the irides are from sequential images, we assume that there are very small rotation differences that will be accounted for when the Hamming distance (explained in Section 3.4) is calculated.

In a similar way the iris mask is also normalized. Examples of iris normalization are depicted in Figure 3.17.

There are different methods used to extract the iris features, the most famous being pioneered by J. Daugman in wavelet domain. His algorithm is based on Gabor wavelets described in Section 3.3.2. Another popular method is R. Wildes' algorithm based on the Laplacian pyramid that aims to find spacial characteristics of the data. It's also worth mentioning Bolle's algorithm that localizes the zeros crossings with one dimensional dyadic wavelet. Gabor wavelet is implemented in this work. 


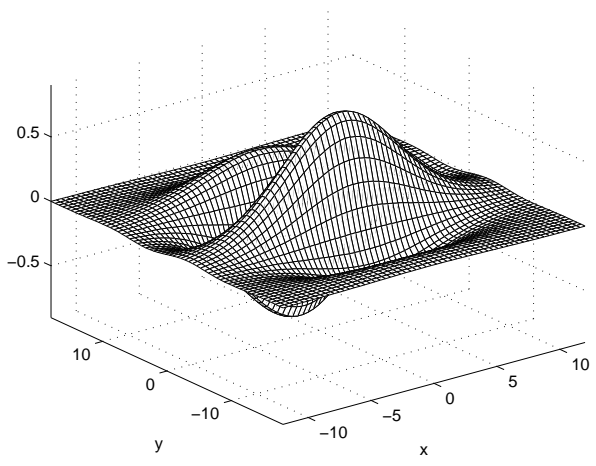

Figure 3.18 Gabor wavelet

\subsubsection{Gabor Wavelets}

Wavelets are mathematical functions that decompose the data in different frequencies at different scales, therefore the data can be represented in the same time in spacial and frequency domains. Two dimensional Gabor wavelets $G(x, y)$ (Figure 3.18) consist of sine/cosine oscillations $S(x, y)$, the carrier, modulated by a 2D Gaussian $W(x, y)$, the envelop [51]: $G(x, y)=S(x, y) \times W(x, y)$. The mathematical expression for the carrier is $S(x, y)=e^{j\left(2 \pi\left(u_{0} x+v_{0} y\right)+P\right)}$, where $u_{0}$ and $v_{0}$ ar spacial frequencies and $P$ is the phase of the sinusoid. The mathematical expression for the envelop is $W(x, y)=K e^{-\pi\left(a^{2}\left(x-x_{0}\right)_{r}^{2}+b^{2}\left(y-y_{0}\right)_{r}^{2}\right)}$, where $\left(x_{0}, y_{0}\right)$ is location of the peak of the function, $K$ is the scale parameter for the amplitude, $a, b$ are the scaling parameters, $r$ represents the rotation operation. According to [51]:

$$
\begin{aligned}
& \left(x-x_{0}\right)_{r}=\left(x-x_{0}\right) \cos (\theta)+\left(y-y_{0}\right) \sin (\theta) \\
& \left(y-y_{0}\right)_{r}=-\left(x-x_{0}\right) \sin (\theta)+\left(y-y_{0}\right) \cos (\theta)
\end{aligned}
$$

where $\theta$ is the rotation angle of the Gaussian. The Gabor wavelet may be seen as two functions, a real and imaginary one, out of phase by 90 degrees. 


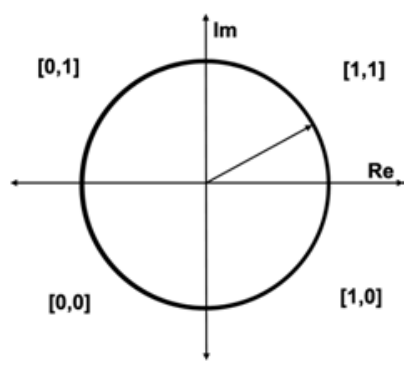

(a) \begin{tabular}{|l}
1000011100110101101011001001001101101101 \\
1000111000101001101111101011001001110010 \\
0100111011001001110010100101110011011011 \\
1110110001011010001110100101111011011011
\end{tabular}

(b)

Figure 3.19 Phase quantization: (a) Four levels represented by the sign of the $I m$ and $R e$ for every quadrant ( 0 - negative, 1 - positive). (b) Example of iris template.

\subsection{Iris Encoding and Matching}

The feature used to encode the iris is the phase vector of the convolution of the normalized iris with the Gabor wavelet through a four level quantization process. The phase quantization is represented by the location of the phase vector in one of the four quadrants in a complex plane as illustrated in Figure 3.19 (a). The values of the two bits are given by the sign of the real and imaginary part of the quadrant where the phase resides. An example of iris template is shown in Figure 3.19 (b).

The matching algorithms use similarity or dissimilarity measures between the two iris templates in order to assess how closely the two templates are to each other. Different measures are convenient for different types of analysis (e.g. numerical data, boolean data, or string data). The iris template is a part of boolean data, and Hamming distance is commonly used for matching. For two $N$ bit iris templates $T_{1}$ and $T_{2}$, the Hamming distance is defined as the sum of all the disagreeing bits divided by $N$. The bits from the non-iris artifacts represented in the iris masks (mask $k_{1}$ and mask $\left._{2}\right)$ are excluded. The mathematical expression of the Hamming distance as a dissimilarity measure for iris templates is as follows: 


$$
H D=\frac{\sum_{i=1}^{N}\left(T_{1_{i}} \oplus T_{2_{i}} \cap \operatorname{mask}_{1_{i}} \cap \operatorname{mask}_{2_{i}}\right)}{N}
$$

\subsection{Sclera Feature Extraction and Matching}

The conjunctival vasculature displayed on the sclera surface is enhanced using selective enhancement filter for lines. The algorithm is described in Chapter 2, Section 2.5. The use of keypoint-based matching method (SURF) described in Chapter 2, Section 2.7.1 was observed to result in the best recognition performance for the scleral patterns. Therefore this method is further used to combine the iris patterns with the sclera patterns.

\subsection{Results}

The ROC and the distribution of scores for all methods employed in this chapter such as SURF, Hamming distance and the score level fusion of iris patterns and scleral patterns, for left and right eye, both gaze directions are depicted in Appendix B. Table 3.1 shows the results of the Hamming distance dissimilarity measure. The best performance of iris recognition is obtained for left-eye-looking-left $\left(L_{-} L\right)$ and right-eye-looking-right $\left(R \_R\right)$ with EER values of less then $1 \%$. In the case of left-eyelooking right $\left(L_{-} R\right)$ and right-eye-looking-left $\left(R_{-} L\right)$ where the light that reaches the

eye is obstructed by the facial structure (the nose) and is directed as much as possible towards the sclera region, the EER values of $3.5 \%$ show a decrease is performance for iris recognition.

Table 3.2 displays the results for interest point matching SURF applied to the pre-processed images of the sclera. The results are promising; the EER values are in 
Table 3.1 The EER (\%) results for iris patterns using Hamming distance.

\begin{tabular}{||l||c|c|c|c||}
\hline \hline Performance measure & L_L $_{-}$ & $\mathbf{L} \_\mathbf{R}$ & $\mathbf{R} \_\mathbf{L}$ & $\mathbf{R} \_\mathbf{R}$ \\
\hline \hline Hamming distance & $0.45 \%$ & $3.5 \%$ & $3.5 \%$ & $0.95 \%$ \\
\hline \hline
\end{tabular}

Table 3.2 The EER (\%) results for scleral patterns when using SURF.

\begin{tabular}{||c||c|c|c|c||}
\hline \hline Performance measure & L_L & L_R & R_L & R_R \\
\hline \hline SURF & $0.225 \%$ & $0.4 \%$ & $0.28 \%$ & $0.2 \%$ \\
\hline \hline
\end{tabular}

the interval $0.2 \%$ to $0.4 \%$.

Table 3.3 shows the EER values for score level fusion of the scleral patterns when using SURF and the iris patterns when using the Hamming distance. For simple sum rule and maximum rule the genuine and impostor distribution of scores have no overlap region with EER value of $0 \%$. For minimum rule, the EER values are in the interval $0.07 \%$ to $0.27 \%$.

SURF scores are similarity scores with integer values greater then 0. Hamming distance measure is a dissimilarity measure with score values between 0 and 0.5 . Before the fusion of the two sets of scores, their values are brought in the interval $[0,1]$. The dissimilarity score is transformed into a similarity score by subtracting its value from 1 . The results of the score level fusion demonstrate the validity of using scleral patterns in non-frontal ocular images of the eye to successfully improve the iris recognition.

The ocular images are processed using Matlab R2010a installed on a OptiPlex 755 computer with an Intel Core 2 vPro processor, $2.99 \mathrm{GHz}$, Windows XP Professional operating system, and $4 \mathrm{~Gb}$ RAM. The average computation times for several procedures are displayed in Table 3.4. 
Table 3.3 The EER (\%) results of the fusion of iris patterns (Hamming distance) and scleral patterns (SURF).

\begin{tabular}{||c||c|c|c|c||}
\hline \hline Performance measure & L_L & L_R & R_L & R_R \\
\hline \hline Simple sum rule & $0 \%$ & $0 \%$ & $0 \%$ & $0 \%$ \\
\hline Maximum rule & $0 \%$ & $0 \%$ & $0 \%$ & $0 \%$ \\
\hline Minimum rule & $0.17 \%$ & $0.07 \%$ & $0.27 \%$ & $0.17 \%$ \\
\hline \hline
\end{tabular}

Table 3.4 Average computation times

\begin{tabular}{||l||l||}
\hline \hline Procedure & $\begin{array}{l}\text { Computation } \\
\text { times }\end{array}$ \\
\hline \hline Specular reflection & $0.65 \mathrm{sec}$ \\
\hline Ocular image segmentation. Sclera-eyelid boundary & $1.67 \mathrm{sec}$ \\
\hline Ocular image segmentation. Pupil segmentation & $3.672 \mathrm{sec}$ \\
\hline Ocular image segmentation. Sclera-iris boundary & $2.631 \mathrm{sec}$ \\
\hline Ocular image segmentation. Iris segmentation & $4.314 \mathrm{sec}$ \\
\hline Ocular image segmentation. Final sclera segmentation & $0.645 \mathrm{sec}$ \\
\hline Blood vessel enhancement & $1.674 \mathrm{sec}$ \\
\hline Blood vessel segmentation & $0.952 \mathrm{sec}$ \\
\hline Minutiae point detection & $0.062 \mathrm{sec}$ \\
\hline \hline
\end{tabular}




\subsection{Summary}

The work presented in this chapter aims to demonstrate the potential of designing a bimodal ocular system that combines sclera biometric with the iris biometric. The ocular images of the first collection of the multispectral database are denoised and further pre-processed to detect and remove the specular reflections. An automatic sclera region segmentation was designed to localize the spatial extent of the iris, sclera and pupil regions in the ocular images. The proposed segmentation algorithm is a combination of region-based and edge-based schemes that exploits the multispectral information. The use of keypoint-based matching was observed to result in the best recognition performance for the scleral patterns. Therefore, Speeded Up Robust Features is used to asses the performance of scleral patterns as biometric cue. Using elliptical unwrapping, the segmented iris is normalized and encoded using 2D Gabor filters. Hamming distance is used as a dissimilarity measure to asses the performance of iris recognition. The sclera scores and the iris scores are combined at a score level using three fusion rules: simple sum rule, maximum rule and minimum rule. The results show an improvement of the EER mainly for the simple sum rule and maximum rule when the genuine and impostor distribution scores are totaly nonoverlapping. This suggests that when looking to the left or looking to the right,

the information gained from the exposed sclera texture may be used to improve the performance of non-frontal iris recognition. 


\section{Chapter 4}

\section{Impact of intra-class variation}

The previous approaches demonstrate the validity of using the sclera texture and the conjunctival vasculature exhibited on its surface as a biometric cue and the benefit gained from the fusion of iris patterns with sclera patterns in non-ideal images of the eye. The ocular images were obtained in a constraint environment, with controlled lighting conditions and distance to the camera. The selection of eight consecutive frames ensured that the viewing angle is approximately the same for all eight images. The results are promising and suggest further insight into addressing the remaining challenges. However, the selection of the consecutive frames resulted in less intraclass variation. The work in this chapter investigates the potential of using the sclera texture and the blood vessels as a biometric cue for ocular images with increased intra-class variation. This is accomplished by using the ocular images of the second collection from the multispectral database presented in Chapter 2, Section 2.1.

The block diagram of the proposed system is shown in Figure 4.1.

After acquisition presented in Chapter 2, Section 2.1, the ocular images are denoised using wavelet transform as presented in Chapter 2, Section 2.2. The specular reflection is detected and removed with the two step algorithm presented in Chapter 3, 


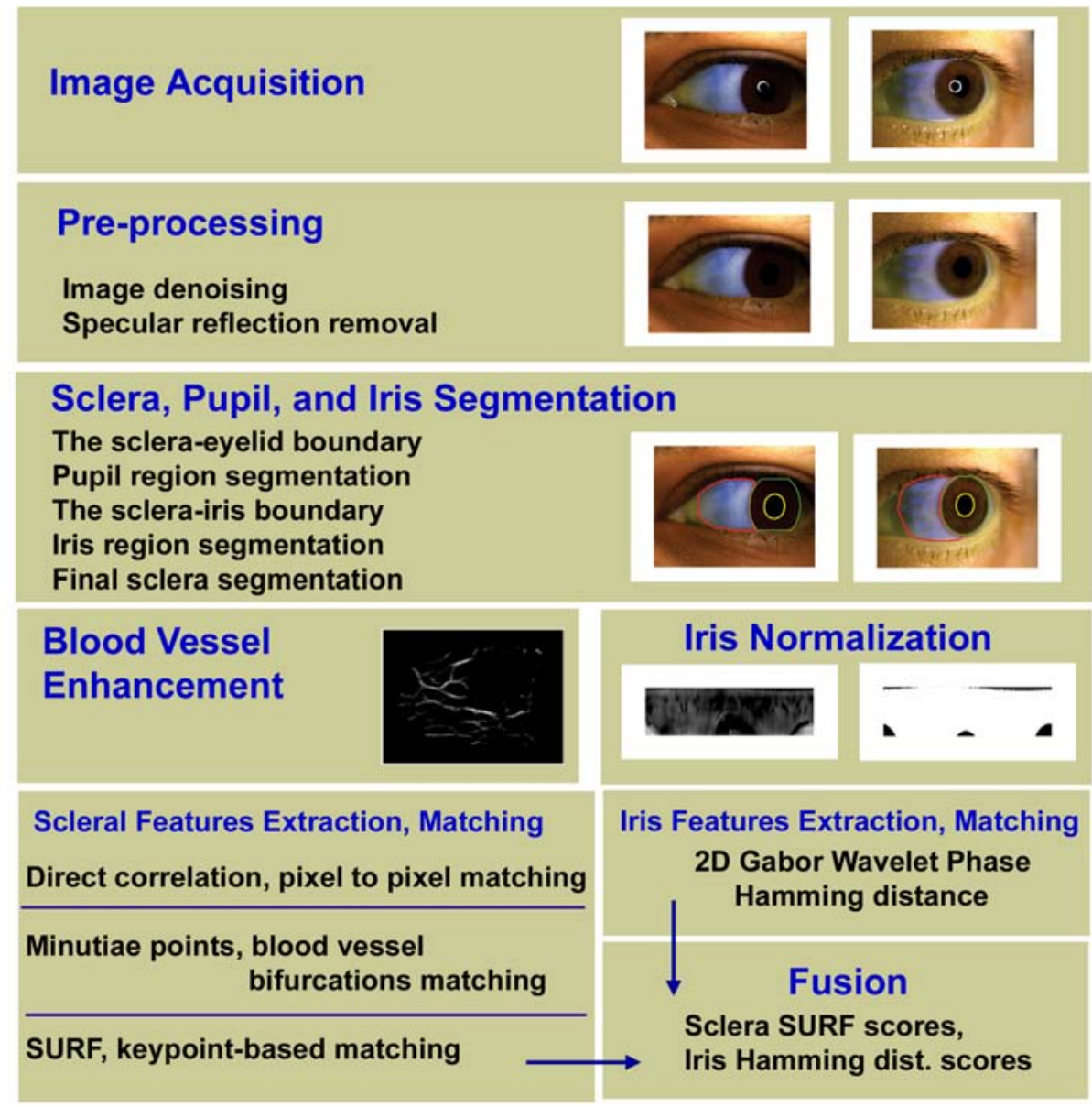

Figure 4.1 Block Diagram 
Section 3.1. The sclera, the pupil and the iris are segmented using the automatic segmentation algorithm, a combination of region-based and edge-based schemes as described in Chapter 3, Section 3.2. The conjunctival vasculature seen on the segmented sclera region is enhanced using the selective enhancement filter for lines as in Chapter 2, Section 2.5 and the pre-processed images are registered as described in Chapter 2, Section 2.6. The three feature extraction and matching techniques mentioned in Chapter 2, Section 2.7, the keypoint-based matching (SURF), pixel to pixel matching (direct correlation matching - CORR, MI, NMI, RMSE, RIU, SSIM) and the bifurcations of the blood vessels matching (minutiae points) are applied to the pre-processed or registered images of the sclera region. Based on elliptical unwrapping algorithm mentioned in Chapter 3, Section 3.3.1, the iris is normalized, and then encoded using 2D Gabor wavelet as in Section 3.3.2 and 3.4. Hamming distance is used to assess the performance of iris recognition. As observed in the previous chapters the keypoint-based matching was observed to result in the best recognition performance for scleral patterns. Therefore the fusion of the iris patterns with the sclera patterns is realized by combining the sclera scores obtained with Speeded Up Robust Features (SURF) and the iris scores obtained with Hamming distance dissimilarity measure.

\subsection{Results}

The ROC and the distribution of scores for all the methods used in this chapter are depicted in Appendix C. As observed from the Table 4.1, for ocular images of the eye with increased intra-class variation there is little change in performance when using keypoint-based matching (SURF) technique. The values of EER are slightly increased from $0.2 \%$ to $0.8 \%$ for right-eye-looking-right $\left(R_{-} R\right)$, but slightly decreased from around $0.2 \%$ to $0.1 \%$ for left-eye-looking-left $\left(L_{-} L\right)$ and right-eye-looking-left 
Table 4.1 The EER (\%) results for scleral patterns when using SURF.

\begin{tabular}{||c||c|c|c|c||}
\hline \hline Performance measure & L_L & L_R & R_L & R_R \\
\hline \hline SURF & $0.175 \%$ & $2.5 \%$ & $0.1 \%$ & $0.8 \%$ \\
\hline \hline
\end{tabular}

Table 4.2 The EER (\%) results for scleral patterns when using minutiae points.

\begin{tabular}{||c||c|c|c|c||}
\hline \hline Performance measure & L_L & L_R & R_L & R_R \\
\hline \hline Minutiae points & $16 \%$ & $15 \%$ & $11.5 \%$ & $16 \%$ \\
\hline \hline
\end{tabular}

$\left(R_{-} L\right)$. This is due to the invariance of SURF to small changes in viewing angle. However, there is a decrease in performance for left-eye-looking-right $\left(L_{-} R\right)$ with 2.1\%. Results are compared with EER values from the Table 4.1.

As presented in Table 4.2, and compared with the values from the Table 2.6, there is an increase in EER values when minutiae points technique is used with around $5 \%$ for left-eye-looking-left $\left(L_{-} L\right)$ and left-eye-looking-right $\left(L_{-} R\right)$. For right-eye-lookingleft $\left(R_{-} L\right)$ the value of EER is approximately the same. For right-eye-looking-right $\left(R \_R\right)$ the EER is increased with $4.5 \%$. In ocular images with increased intra-class variation, the performance of sclera biometric when using minutiae points technique is decreased. The location of the bifurcations of the blood vessels are not invariant to the changes in the viewing angle mainly on a curved surface such as the eyeball.

The Table 4.3 contains the EER values for ocular images when direct correlation methods are used. Mutual information $(M I)$, normalized mutual information $(N M I)$, and ratio-image uniformity $(R I U)$ decrease in performance compared with the values displayed in the Table 2.7. An improved performance is observed for structural similarity index measure $(S S I M)$. The EER for root mean square error $(R M S E)$ and correlation $(C O R R)$ methods are approximately the same with the 
Table 4.3 The EER (\%) results for scleral patterns when using different correlation methods.

\begin{tabular}{||c||c|c|c|c||}
\hline \hline Performance measure & L_L & L_R & R_L & R_R \\
\hline \hline RMSE & $9.5 \%$ & $4.5 \%$ & $4.3 \%$ & $1.5 \%$ \\
\hline CORR & $6 \%$ & $1.1 \%$ & $1.1 \%$ & $0.21 \%$ \\
\hline MI & $10 \%$ & $14.5 \%$ & $12 \%$ & $12 \%$ \\
\hline NMI & $9 \%$ & $11 \%$ & $7 \%$ & $5.75 \%$ \\
\hline RIU & $15 \%$ & $14 \%$ & $12 \%$ & $14 \%$ \\
\hline SSIM & $5 \%$ & $1.1 \%$ & $0.9 \%$ & $0.9 \%$ \\
\hline \hline
\end{tabular}

exception of left-eye-looking-left $\left(L_{-} L\right)$ when an increase of $8 \%$ and $5.9 \%$ respectively is observed. The decrease in performance for all techniques for left-eye-looking-left compared with right-eye-looking-left and right-eye-looking-right may be explained by the position of the ophthalmologist's slit-lamp mount that did not allow a better adjustment of the position of the camera and the source of light when images of the left eye were collected. The mount was positioned having the office wall to the right, so that the participant had the wall on the left side. This was not apprehended at the data collection time.

The EER values for the Hamming distance are compared with the values from the Table 4.4. The values for the right eye, both gaze directions show an improved performance explained by a better illumination and the focus of the camera on the iris region Section 2.1. The values for the left eye do not show an increase or decrease in performance. Similar with the results obtained when direct correlation methods are used, the lack of improvement may be explained by the data collection setup.

As expected the combination of the sclera biometric with the iris biometric presents good results for all three score level fusion rules. For simple sum rule and maximum 
Table 4.4 The EER (\%) results for iris patterns using Hamming distance.

\begin{tabular}{||l||c|c|c|c||}
\hline \hline Performance measure & L_L & L_R & $\mathbf{R} \_\mathbf{L}$ & $\mathbf{R} \_\mathbf{R}$ \\
\hline \hline Hamming distance & $0.55 \%$ & $3.8 \%$ & $2.2 \%$ & $0.02 \%$ \\
\hline \hline
\end{tabular}

Table 4.5 The EER (\%) results of the fusion of iris patterns (Hamming distance) and scleral patterns (SURF).

\begin{tabular}{||c||c|c|c|c||}
\hline \hline Performance measure & $\mathbf{L}_{-} \mathbf{L}$ & $\mathbf{L} \_\mathbf{R}$ & $\mathbf{R} \_\mathbf{L}$ & $\mathbf{R} \_\mathbf{R}$ \\
\hline \hline Simple sum rule & $0 \%$ & $0 \%$ & $0 \%$ & $0 \%$ \\
\hline Maximum rule & $0 \%$ & $0 \%$ & $0 \%$ & $0 \%$ \\
\hline Minimum rule & $0 \%$ & $2.5 \%$ & $0 \%$ & $0 \%$ \\
\hline \hline
\end{tabular}

rule, the genuine and impostor distribution of scores are totaly non-overlapped. EER value is $0 \%$ for both eyes, both gaze directions. This suggests that the fusion of iris patterns and scleral patterns may be used with success to improve iris recognition in data sets with intra-class variation.

\subsection{Summary}

In the previous chapters the potential of using the sclera texture and the blood vessels exposed on its surface is assessed using the first collection of the multispectral database. The ocular images are obtained in constraint environment, with controlled lighting conditions and distance to the camera. The selection of eight consecutive frames ensured that the viewing angle is approximately the same for all eight images, but resulted in less intra-class variation. The work in this chapter investigates the potential of using the sclera texture and the blood vessels as a biometric cue for ocular images with increased intra-class variation. This is accomplished by using the ocular images of the second collection from the multispectral database. The auto- 
matic segmentation algorithm is used to localize the iris, sclera and pupil regions. Conjunctival vasculature is enhanced using the selective line enhancement filters for lines and pre-processed images of the sclera are registered as described in previous chapters. The three feature extraction methods, keypoint-based matching, direct correlation methods and minutiae points are applied to the pre-processed images of the sclera. The results demonstrate an increase of EER for direct correlation methods and minutiae points method. This is explained by the variance of these methods to changes in the viewing angle. On the other hand, keypoint-based method SURF exhibits the same good results. This is explained by the fact that SURF is invariant to small changes in the viewing angle. Further the score level fusion of scleral patterns and iris patterns when using SURF and Hamming distance, presents the same good results, proving that the use of scleral patterns combined with iris patterns may improve the iris recognition in non-frontal images of the eye. 


\section{Chapter 5}

\section{Sclera recognition using low}

\section{resolution visible spectrum images}

\section{$5.1 \quad$ Visible spectrum data set}

The SONY CyberShot DSC F717 (5 megapixels) was used to capture color images of the eye ${ }^{1}$. Each subject was asked to move their eyes in the following manner with respect to the optical axis of the camera: frontal, upward, to the left and to the right. Thus different regions of the sclera where represented in the ensuing pictures. These color images (RGB) were collected in two sessions. The first session had 2400 images from 50 subjects, and the second session had 816 images from 17 of the original 50 subjects. Images were captured from both eyes at three different distances: 1 foot considered as near distance images, 9 feet as medium distance images, and 12 feet as far distance images. For each eye, 2 images were collected per gaze at each distance. Figure 5.1 displays the four gaze directions for near distance.

\footnotetext{
${ }^{1}$ Collected at University of Missouri, Kansas City
} 


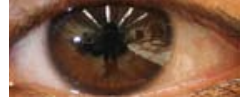

(a)

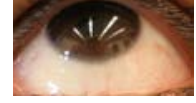

(b)

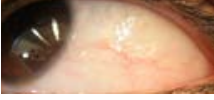

( c)

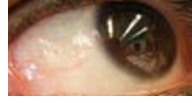

(d)

Figure 5.1 Near images of the eye where the subject is: a) looking straight ahead, b) looking up, c) looking left, d) looking right.

\subsection{Sclera region segmentation}

Accurately segmenting the sclera from the eye image is very important for further processing as stated in Chapter 2, Section 2.4. A semi-automated technique is used for this purpose. The proposed technique first applies an automated clustering method whose output is subsequently refined by manual intervention. Each pixel is represented as a three-dimensional point in a Cartesian coordinate system based on its primary spectral components of red, green and blue. The k-means clustering algorithm is used to partition the pixels into three categories: the sclera, the iris, and the background (Figure 5.2 (b)). Since the sclera is typically whiter than the rest of the eye, such a procedure is expected to work well in separating the scleral pixels from the rest of the image. The pixels pertaining to the sclera region are determined as the cluster with the largest Euclidean distance from the origin of the coordinate system to its centroid. The pixels belonging to the iris region are determined as the cluster with the smallest Euclidean distance from the origin of the coordinate system to its centroid. A mask for the iris region and a mask for the sclera region comprise the output of the clustering method. Entries marked as 1 in the masks denote the pixels assigned to the particular cluster (iris or sclera). The largest connected region is selected in both masks. Due to the image characteristics of illumination and specular reflection, it is possible for some pixels from the sclera to not be assigned to the proper cluster, thereby appearing as holes in the sclera mask. In order to eliminate 


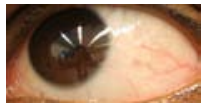

(a)

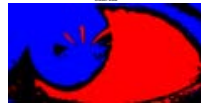

(b)

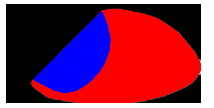

(c)

Figure 5.2 Segmenting the sclera from two different eye images, displayed by column: a) Original image, b) Segmented sclera region based on RGB values $($ red $=$ sclera region, blue $=$ iris region, black $=$ the background $) \mathrm{c}$ ) Convex hull of the sclera (blue+red) containing a portion of the iris (blue)

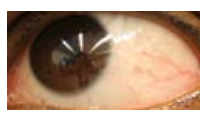

(a)

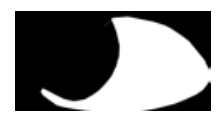

(b)

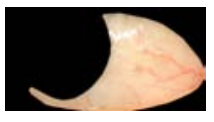

(c)

Figure 5.3 Segmenting the sclera of two different eye images: a) Original image, b) Sclera mask, c) Segmented sclera region.

these holes and to smooth the contour of the sclera mask, its convex hull is considered (Figure 5.2(c)). This, however, means that pixels pertaining to the iris cluster may be included in the sclera mask. To address this, we first locate the pixels within the convex hull of the sclera region belonging to the iris cluster. Next, we remove the convex hull of the located pixels from the convex hull of the sclera region. The output of the process is a binary mask (Figure $5.3(\mathrm{~b})$ ), which when imposed on the original image, will identify the region of interest corresponding to the sclera (Figure 5.3 (c)). On examining the segmented sclera region, we observed that in some images, a small portion of the lower eyelid was erroneously included. To address this issue, the mask is manually corrected for such images, thereby eliminating the lower eyelashes. Table 5.1 records the number of images for which manual correction of the segmented sclera was needed. 
Chapter 5 Sclera recognition using low resolution visible spectrum images

Table 5.1 Manual correction statistics of segmented sclera

\begin{tabular}{|c|c|c|c|c|c|}
\hline \multirow[t]{2}{*}{ Gaze } & \multirow[t]{2}{*}{ Distance } & \multicolumn{2}{|c|}{ Left eye } & \multicolumn{2}{|c|}{ Right eye } \\
\hline & & $\begin{array}{l}\text { automatic } \\
\text { segmentation } \\
\text { and manual } \\
\text { correction }\end{array}$ & $\begin{array}{c}\text { only } \\
\text { automatic } \\
\text { segmentation }\end{array}$ & $\begin{array}{c}\text { automatic } \\
\text { segmentation } \\
\text { and manual } \\
\text { correction }\end{array}$ & $\begin{array}{c}\text { only } \\
\text { automatic } \\
\text { segmentation }\end{array}$ \\
\hline \multirow{3}{*}{ Left } & near & 61 & 73 & 42 & 92 \\
\hline & medium & 49 & 85 & 36 & 98 \\
\hline & far & 43 & 91 & 39 & 95 \\
\hline \multirow{3}{*}{ Right } & near & 54 & 80 & 56 & 78 \\
\hline & medium & 53 & 81 & 51 & 83 \\
\hline & far & 35 & 99 & 44 & 90 \\
\hline
\end{tabular}

\subsection{Specular reflection}

\subsubsection{General considerations}

Specular reflections may provide valuable information about the shape of the object

and its location with respect to the light source. However, they can cause problems for image processing algorithms that may erroneously consider these specularities as pixels of interest during the process of segmentation resulting in spurious results. Localization of specularities in images is very important and requires a good understanding of the reflection of light, a complicated process that depends on the material of the object under consideration, the roughness of its surface, the angle of illumination, the angle of viewing, and the wavelength of light.

Specular reflections on the sclera have different topologies, sizes and shapes that cannot be described by a single pattern. Their pixel intensity values are distinctively 


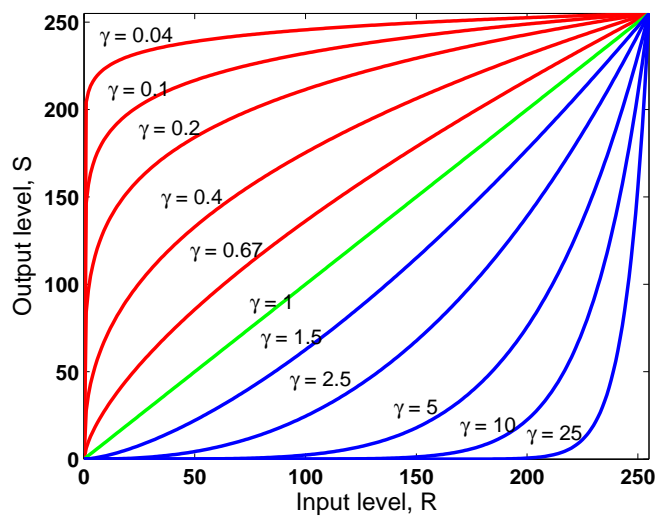

Figure 5.4 Plots of the equation 5.1 for various values of $\gamma ; \mathrm{c}=1$ in all cases

high, and exhibit a large variation both within the same image and across multiple images. Different approaches for specular reflection detection and removal have been proposed in the literature [52], [53], [54], [55]. The algorithm for specular reflection consists of three main steps: detection and localization of specular reflection, construction of specular reflection mask, and exclusion of the region containing the specular reflection from the sclera region.

\subsubsection{Detection of specular reflection}

If original images of sclera containing specular reflection were to be further processed, as explained in the following sections, the edges of the specular reflection region may appear as spurious blood vessels in the enhanced image. The algorithm to detect specular reflections is based on the power law transformation as applied to pixel intensities in the color image. Power law transformations have the basic form:

$$
S=c * R^{\gamma},
$$

where $\mathrm{c}, \gamma$ are positive constants, $\mathrm{R}$ is the input pixel intensity, and $\mathrm{S}$ is the output intensity. As shown in Figure 5.4, by simply varying $\gamma$ we obtain a family of possible 
transformation curves. For $\gamma>1$, the power - law curves map a narrow range of light input values into a wider range of output values. For $\gamma<1$, the power - law curves map a narrow range of dark input values into a wider range of output values. In order to detect specularities, we consider $\gamma$ an integer in the range $[1,10]$. The detection of the specular reflection is as follows.

1. Convert the RGB image to the HSI (hue, saturation, illumination) color space.

2. Consider the illumination component of the HSI space as the input image R in equation 5.1.

3. Compute the output image $\mathrm{S}$ for different $\gamma$ values using equation 5.1. Fig. 5.5 (a) displays results for gamma $=3$.

4. Compute the histogram for each image $\mathrm{S}$ as seen in Fig. 5.5 (b).

5. Compute the filtered histogram for each image $\mathrm{S}$ using the moving average $[1 / 3$ $1 / 31 / 3]$ filter as seen in Fig. 5.5 (c).

6. Compute the slope $\theta$ of the filtered histogram.

7. For the filtered histogram corresponding to each $\gamma$, find the first negative $\theta\left(\theta_{\gamma}\right)$ and its corresponding intensity value, $S_{\gamma}$, as a potential threshold value for detecting specular reflection.

8. Examine the distribution of $\theta_{\gamma}$ as a function of $\gamma$ to select $\gamma_{o p t}, \gamma_{\text {opt }}=\arg \max (\mid$ $\left.\theta_{\gamma}-\theta_{\gamma-1} \mid\right)$. Figure 5.6 shows $\gamma_{\text {opt }}=5$; for near distance images, the threshold to detect specular reflection is selected as the mean of all thresholds values found for $\gamma, 5 \leq \gamma \leq 10$.

9. Use the threshold value found to obtain a binary mask for isolating specular reflection. 


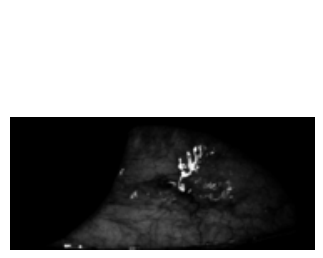

(a)

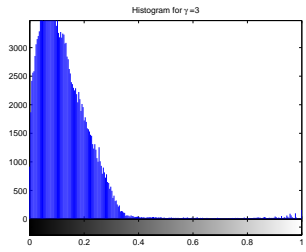

(b)

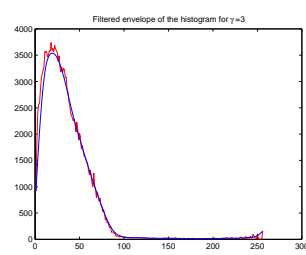

(c)

Figure 5.5 Detection of specularities. Examples for $\gamma=3$ : (a) Illumination component of HSI sclera image; (b) Histogram of the illumination component; (c) Filtered envelop of the histogram

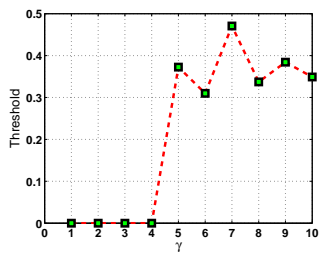

Figure 5.6 Example of threshold values for different values of $\gamma$

Fig.5.7 shows the results of specular reflection detection.

\subsection{Segmented sclera image without specular re- flection}

The segmented sclera image without specular reflection is obtained as follows:

1) Use sclera mask and specular reflection mask to obtain the final sclera mask

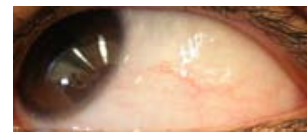

(a)

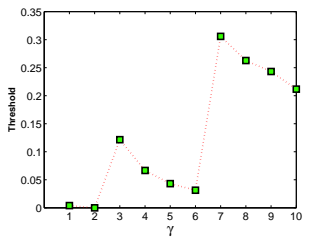

(b)

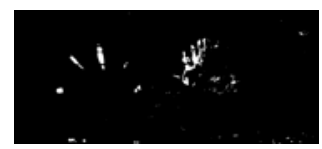

(c)

Figure 5.7 Detecting specularities: a) Original image, b) Threshold values for $1 \leq \gamma \leq 10$ c) Specular reflection mask 


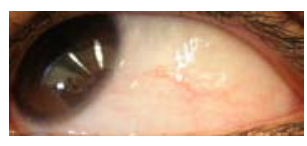

(a)

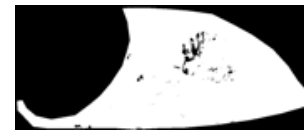

(b)

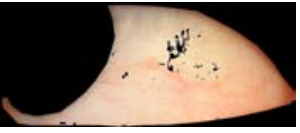

(c)

Figure 5.8 Segmenting the sclera after removing specularities: a) Original image, b) Specular reflection mask c) Segmented sclera without specular reflection

without specular reflection.

2) Superimpose the final mask on the RGB image to obtain segmented sclera without specular reflection (Fig.5.8).

\subsection{Image Pre-processing}

To improve segmentation of the blood vessel patterns, the segmented sclera image is pre-processed in two consecutive steps as described in [27]. In the first step, we build the RGB image from the three components, red, green and blue obtained in section 2.1.1. The RGB image is converted to $\mathrm{L}^{*} \mathrm{a} * \mathrm{~b}$ color space. Contrast-limited adaptive histogram equalization (CLAHE) [56] is applied to the luminance component $\mathrm{L}^{*}$. The algorithm divides the entire image in small square regions called tiles. Each tile is enhanced using histogram equalization. This induces artificial boundaries between tiles that are removed using bilinear interpolation. The $\mathrm{L}^{*} \mathrm{a} * \mathrm{~b}$ image is converted back to RGB color space (Figure 5.9 (b)).

In order to obtain the best results for vein segmentation, an examination of the three components (red, blue and green) of the enhanced colored sclera images, suggests the use of the green component that has the best contrast between the blood vessels and the background. In order to improve sensitivity to vein detection and segmentation, we use a selective enhancement filter for lines, as described in Chapter 2, 


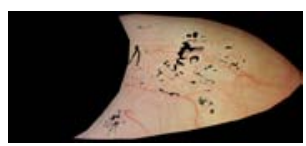

(a)

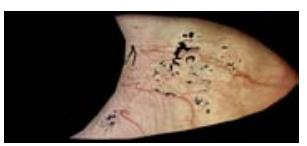

(b)

Figure 5.9 Image Enhancement:(a) Original sclera vein image, (b) Enhanced sclera vein image.

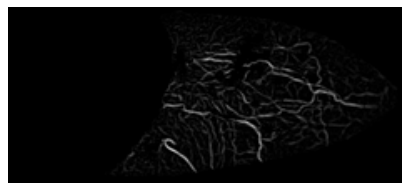

Figure 5.10 Example of pre-processed sclera vein image.

Section 2.5. Results are displayed in Figure 5.10.

After enhancement, images are registered using the algorithm presented in Chapter 2, Section 2.6.

\subsection{Matching Results}

The similarity between two sclera images is assessed using cross-correlation between regions of the sclera that do not include the specular reflections from both images. Results are displayed in Figure 5.11. In the case of low resolution, unconstrained images, the initial analysis indicates that an EER of $\sim 25 \%$ has been obtained on a subset of images belonging to the left-eye-looking-left, near-distance category. An assessment of the results suggests that the large intra-class variation is caused by the following factors: (a) the curved surface of the eye that cannot be modeled using a linear surface; (b) the large range of viewing angles; (c) the glossy surface of the sclera that reflects light; (d) eyelashes that obstruct the sclera region and can be incorrectly perceived as vasculature patterns; and (e) the presence of less prominent veins that can degrade performance. 


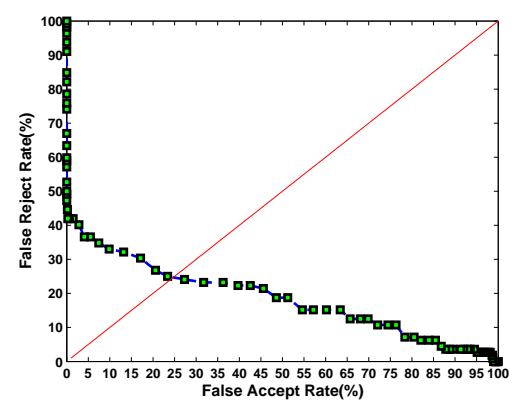

Figure 5.11 ROC curve indicating the results of matching

\subsection{Summary}

This work investigates the usability of the sclera texture and the vasculature patterns in visible spectrum images (RGB) as a biometric cue. The images of the eye are collected in unconstrained lighting conditions, distances and viewing angles. The purpose is to evaluate the matching performance and to examine how the results vary when moving from high to low resolution, from constrained to unconstrained environment. The segmentation of the sclera region based on k-means clustering method was presented; specular reflection detection and conjunctival vasculature enhancement were described; and direct correlation method for matching performance was used. There are several challenges associated with processing these images. These issues are related to: (a) the curved surface of the eyeball; (b) harsh ambient lighting

resulting in significant specularities; (c) the large range of viewing angles; (d) eyelashes that obstruct the sclera region and can be incorrectly perceived as vasculature patterns; and (e) the presence of less prominent veins that can degrade performance. The results reflect the challenges that further have to be conquered to consider the sclera texture and the vasculature patterns as a biometric cue. 


\section{Chapter 6}

\section{Discussions and Conclusions}

Among different biometric modalities, iris recognition has gained popularity in the last decade due to its reliability, accuracy, and stability over long periods of time. However, it has been noted that when the gaze direction is non-frontal in regards to the imaging device, the performance of iris recognition degrades considerably. The idea of using the sclera surface as a biometric modality was developed to compensate the loss of information in non-frontal images of the iris. There is an academic interest in sclera biometrics as an individual and independent component of biometric science. Based on the patent [24] approved in 2008, the work in this dissertation investigates the novel use of the sclera texture and blood vessels seen on its surface as a biometric cue. This new modality is presented as a potential part of the ocular biometric entity that reunites all the biometric modalities related to the eye and its surrounding region. Iris patterns are better observed in the NIR spectrum, while the blood vessels exposed on the sclera surface are better discerned in the visible spectrum. Therefore, multispectral images of the eye are used to ensure that both the iris and the sclera region are successfully imaged. A high resolution multispectral database consisting of two different collections - the first of 103 subjects and the second of 31 subjects - was 
assembled to initiate the research in this field. The images were collected within specific constraints that included stable lighting, consistent distance, and similar viewing angles. The pre-processing and post-processing of the ocular images required image denoising; specular reflection detection and removal; automatic segmentation of the sclera, the iris, and the pupil;blood vessel enhancement; and image registration. To evaluate and assess the performance of the sclera texture as a biometric modality, first we had to find the feature extraction methods and matching algorithms that better characterized this biometric. The study of the sclera texture as a biometric modality was accomplished through four approaches.

1. The performance of sclera texture is evaluated by using three feature extraction and matching schemes: SURF, a keypoint-based matching method; direct correlation methods such as correlation, mutual information, normalized mutual information, root mean square error, structural similarity index measure, and ratio-image uniformity, such as pixel to pixel matching; and minutiae point matching, which mark the locations of bifurcations of blood vessels visible on the sclera region. The score level fusion of minutiae with each of the direct correlation methods is evaluated using the simple sum rule, maximum rule, and minimum rule (Chapter 3).

2. After establishing the potential use of sclera as a biometric cue, the matching performance of the fusion of sclera patterns (scores obtained with SURF technique) with iris patterns (scores obtained with Hamming distance) is evaluated by using three score level fusion rules: simple sum rule, maximum rule, and minimum rule (Chapter 4).

3. The matching performance of the sclera texture as a potential biometric modality is evaluated for data sets with intra-class variation. The methods used for 
feature extraction and matching schemes are the ones used in the first approach.

The performance of the fusion of the sclera biometric (using SURF technique) and the iris biometric (using Hamming distance) is also evaluated (Chapter 5).

4. The evaluation of sclera texture as a biometric in unconstrained, low resolution visible spectrum images. (Chapter 6).

The first approach investigated the feasibility of using multispectral conjunctival vasculature in an ocular biometric system. Due to its relative novelty as a research topic, this paper mainly covers the challenges imposed when acquiring and processing sclera images and should therefore be treated as a gateway to further exploration of the sclera veins as a biometric modality. A new sclera segmentation method was presented and different feature extraction and matching techniques were used to represent this biometric. The matching results using all the methods indicate lower values of EER for left-eye-looking-left $\left(L_{-} L\right)$ and right-eye-looking-right $\left(R_{-} R\right)$ compared with right-eye-looking-left $\left(R_{-} L\right)$ and left-eye-looking-right $\left(L_{-} R\right)$. This is because the facial features (such as the nose) partially obstruct the light directed to the left eye when looking right and to the right eye when looking left. In such cases the $E E R$ is improved using fusion methods. The best accuracy $\left(E E R<0.8 \%\right.$ for $L_{-} L, R_{-} R$, and $E E R<1.8 \%$ for $\left.L_{-} R, R_{-} L\right)$ is obtained when interest-point detection (SURF) is used. This is because SURF utilizes the entire sclera region, including the vasculature patterns; further it is not sensitive to small variations in the viewing angle, affine deformations, and the color shades. Direct correlation measures also provide good results, mainly when correlation $\left(E E R<1.3 \%\right.$ for $L_{-} L, R_{-} R$, and $E E R<4.8 \%$ for $\left.L_{-} R, R_{-} L\right)$ and normalized mutual information $\left(E E R<1.7 \%\right.$ for $L \_L, R \_R$, and $E E R<5.3 \%$ for $\left.L_{-} R, R_{-} L\right)$ are used. Like the SURF method, direct correlation measures use the entire sclera surface including the conjunctival vasculature, but in 
contrast are sensitive to changes in the viewing angle and illumination. Results could be improved with a better blood vessel enhancement method. The minutiae based method presents an EER in the range [9.5\%,12\%] for both eyes and both gaze directions. The performance is greatly impacted by the presence of more or less prominent veins, by the percentage of successfully segmented blood vessels and the accuracy with which the centerline of the blood vessels is found. The perceived shape and tortuosity of the blood vessels are influenced by the small changes in the viewing angle. This method can be improved by accurately segmenting the blood vessels resulting in a higher percentage of segmented blood vessels containing finer veins. The fusion of direct correlation measure scores with minutiae scores is performed in an attempt to boost the matching performance. In the case of left-eye-looking-right and right-eyelooking-left, where the light that reaches the eye is obstructed by the facial structure and the curvature of the eyeball, the EER is lowered when the sum rule is used for fusing minutiae based scores with each of the direct correlation scores, except when using mutual information and normalized mutual information.

The second approach investigated the benefits of combining the iris biometric with the sclera biometric. The first approach demonstrated that SURF technique resulted in the best recognition performance for scleral patterns. Therefore, this technique was used to further combine the sclera patterns with iris patterns. Among different evaluation techniques for iris biometric, Hamming distance is one of the most popular. Three techniques were used to fuse the SURF scores with the Hamming distance scores: the simple sum rule, maximum rule, and minimum rule. As observed, the combination of the two biometric modalities resulted in improved iris recognition performance, especially when the simple sum rule or maximum rule was used. The EER had the value of $0 \%$ for both eyes and both gaze directions, and there was no overlap between the genuine and impostor distribution of scores. The performance of 
iris recognition was also improved when using the minimum rule. The values of the EER, which were obtained using Hamming distance, were between $0.45 \%$ and $3.5 \%$. After the fusion of the iris and the sclera patterns, the EER values were limited to the interval $0.2 \%$ to $0.4 \%$. The results suggested that iris recognition performance would be improved by the fusion with sclera recognition.

The third approach investigated the potential of using the sclera patterns as a biometric cue in the presence of intra-class variation. However, sclera recognition exhibited the same performance with or without the presence of intra-class variation when keypoint-based method SURF was used. The EER values were contained within the interval $0.1 \%$ to $0.8 \%$ for left-eye-looking-left, right-eye-looking-right, and righteye-looking-left. Only the value of EER for the left-eye-looking-right was slightly higher, at $2.5 \%$. SURF technique is invariant to small changes in the viewing angle. By comparison, the direct correlation techniques and minutiae points method are not invariant to the changes in the viewing angle. Therefore, a decrease in the performance of sclera recognition was observed when the variant methods were used. The EER values for minutiae points were constrained to the interval $11.5 \%$ to $16 \%$, which were also higher values when compared with those from the Table 2.6. The performance of iris recognition in this approach was more positive than when compared with the values of EER obtained in the second approach. This is explained by a better illumination of the eye and the focus of the camera on the iris region. Similarly, the iris recognition performance for ocular images with intra-class variation was increased as a result of the fusion of iris patterns with sclera patterns.

The fourth and the last approach investigated the usability of the sclera texture and the vasculature patterns in visible spectrum images (RGB) as a biometric cue. The images collected in an unconstrained environment exhibited a large range of viewing angles, specular reflections of different sizes, topologies, and locations. The 
correlation was used for matching performance. For near distance, left-eye-lookingleft, the EER is $25 \%$. A future step is to address the challenges encountered when acquiring and processing images of the sclera collected in unconstrained environment.

The following publications were generated as a consequence of this research:

1. S. Crihalmeanu, A. Ross,Multispectral Scleral Patterns for Ocular Biometric Recognition, Pattern Recognition Letters, In press 2012, http://dx.doi.org./10.1016/j.patrec.2

2. S. Crihalmeanu and A. Ross, On the Use of Multispectral Conjunctival Vasculature as a Soft Biometric, WACV, Kona USA, January 2011

3. S. Tankasala, P.Doynov, R. Derakhshani, A. Ross and S. Crihalmeanu, Classification of Conjunctival Vasculature using GLCM Features, ICIIP, Shimla India, November 2011

4. S. Crihalmeanu, A. Ross and R. Derakhshani, Enhancement and Registration Schemes for Matching Conjunctival Vasculature, ICB, Alghero Italy, June 2009

5. R. Derakhshani, A. Ross and S. Crihalmeanu, A new Biometric Modality Based On Conjunctival Vasculature, ANNIE, Saint Louis USA, November 2006

\subsection{Future work}

The results obtained in the four approaches described in this dissertation demonstrate the potential of using the sclera surface and the conjunctival vasculature for recognition and reflect the challenges that further have to be conquered to consider the sclera texture and the vasculature patterns as a biometric cue. The results suggest that more work is needed in this area, such as evaluating the sclera in non-frontal 
images of the eye using texture analysis techniques described in [57]: color texture analysis, random texture analysis, hierarchical texture, etc. Another approach could be the study of segmented blood vessels as open curves, their tortuosity and thickness (more or less prominent blood vessels), and the changes that occur with an inconsistent viewing angle. The detection of the eyelashes may help improve the sclera region segmentation. Other issues that may be addressed are related to lessening the constrains of the environment; for example, by evaluating the sclera surface in ocular images with the entire sclera exposed (wide open eye) or partially occluded sclera region, and evaluating the matching performance of the sclera texture under different lighting conditions and different viewing angles. The age of the subject and the chemicals the eye has come in contact with both greatly influence the appearance of the sclera texture. These changes may have a significant effect not only on the matching performance, but also on the segmentation process. It is well known that an improper segmentation will also influence the performance matching. Little research for iris recognition using multispectral images is published. A more in depth exploitation of the multispectral information may support a more accurate segmentation of the iris and sclera region, and feature extraction and matching algorithms. The answers to all these issues lead to the innovative idea of customizing imaging systems for sclera. As for any other biometric system, a quality measure has to be found in order to address the problem of failure to acquire.

In this dissertation the possibility of utilizing the scleral patterns in conjunction with the iris for recognizing ocular images exhibiting non-frontal gaze directions was established. 
Appendix A

Methods for sclera patterns

matching. The ROC and the

distribution of scores. Data

Collection 1 
Chapter A Methods for sclera patterns matching. The ROC and the distribution of scores. Data Collection 1

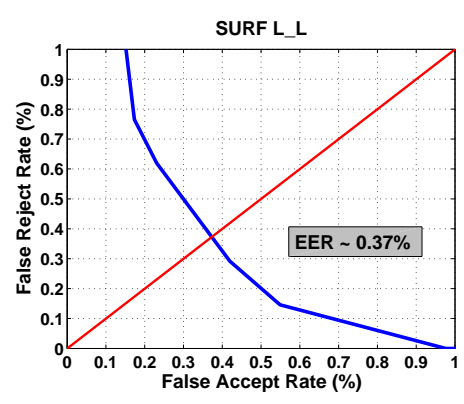

(a)

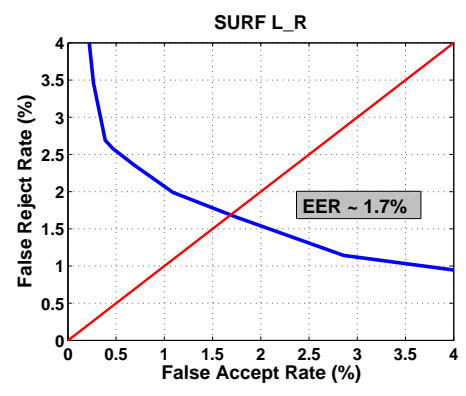

(c)

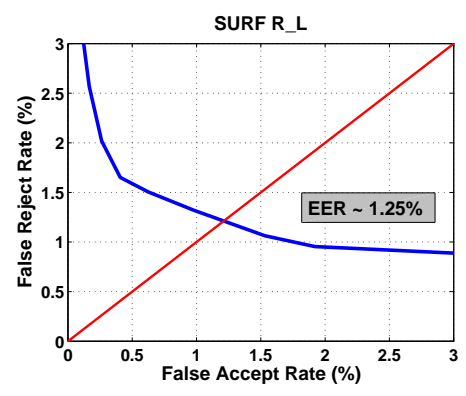

(e)

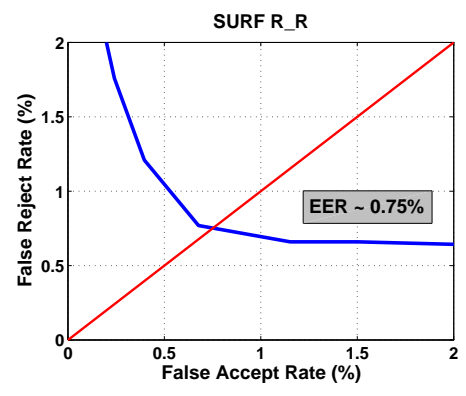

$(g)$

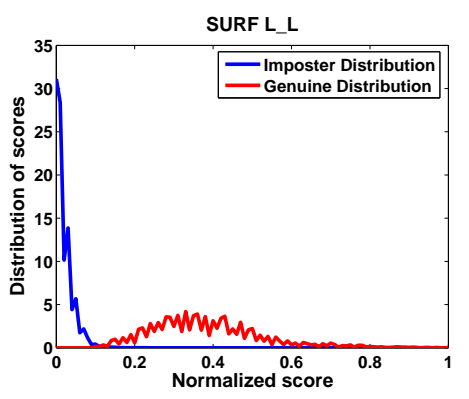

(b)

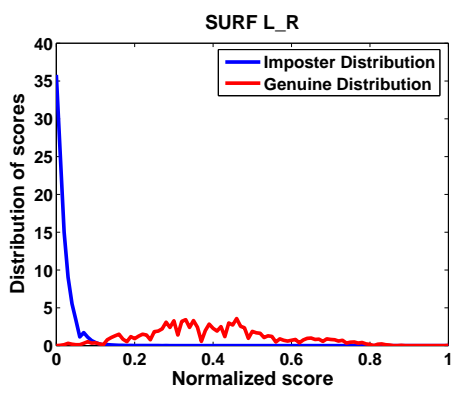

(d)

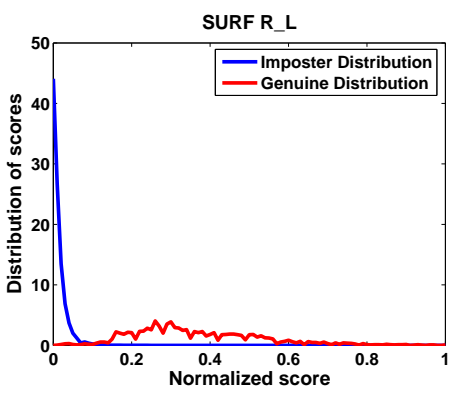

(f)

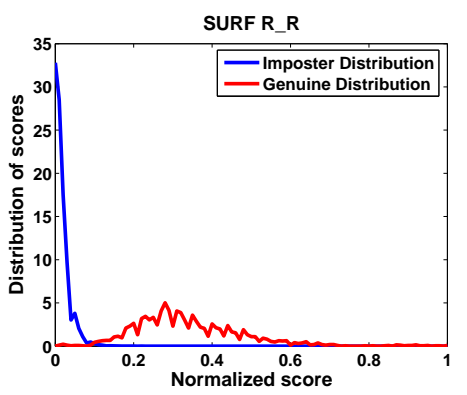

(h)

Figure A.1 Data collection 1. The ROC and the distribution of scores for the SURF technique. 
Chapter A Methods for sclera patterns matching. The ROC and the distribution of scores. Data Collection 1

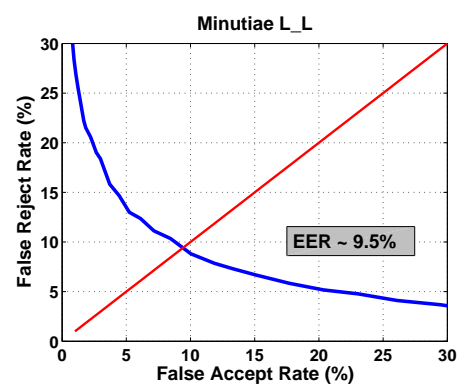

(a)

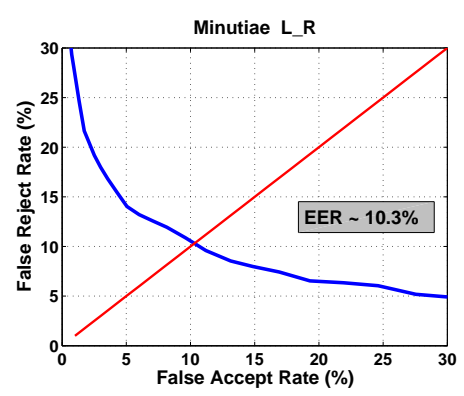

(c)

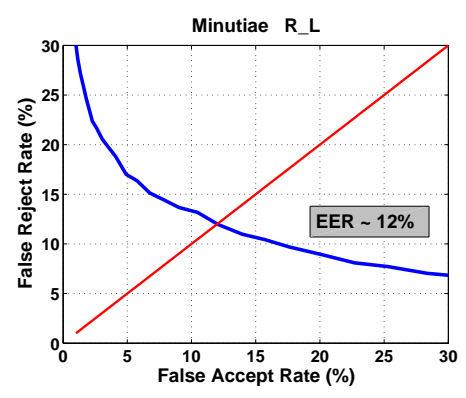

(e)

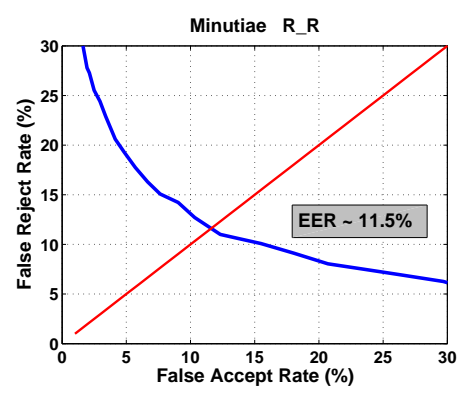

(g)

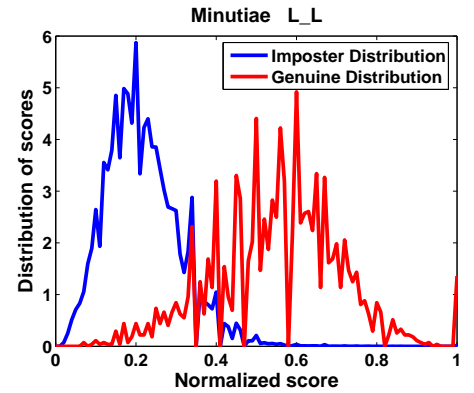

(b)

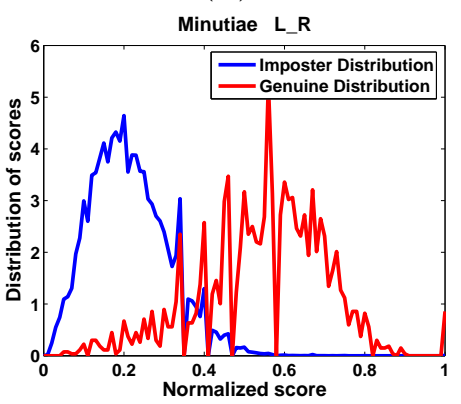

(d)

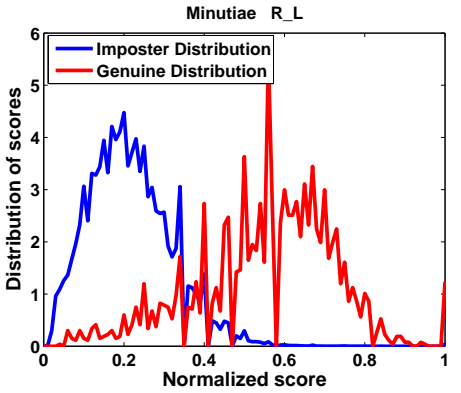

(f)

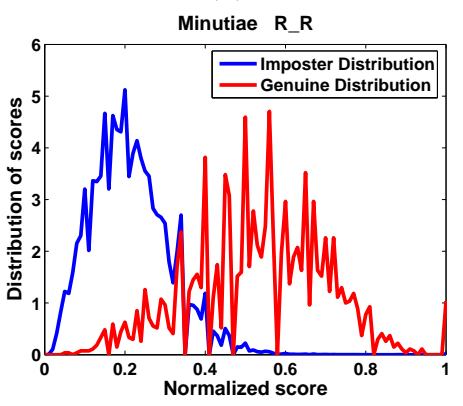

(h)

Figure A.2 Data collection 1. The ROC and the distribution of scores for the minutiae-based matching technique. 
Chapter A Methods for sclera patterns matching. The ROC and the distribution of scores. Data Collection 1

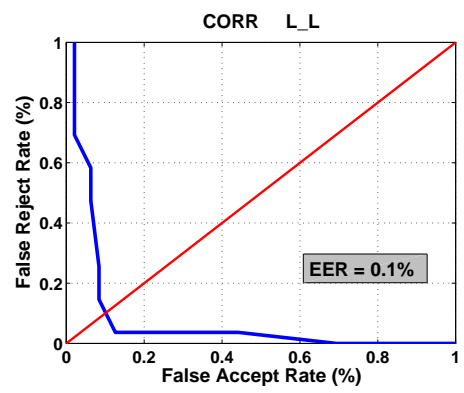

(a)

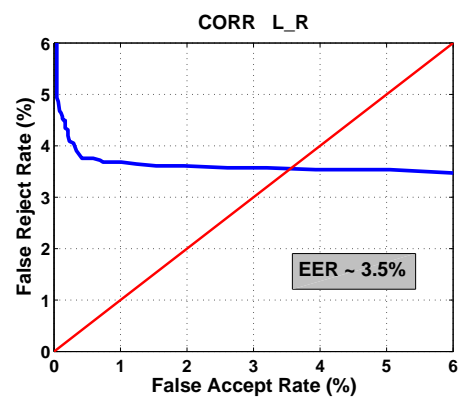

(c)

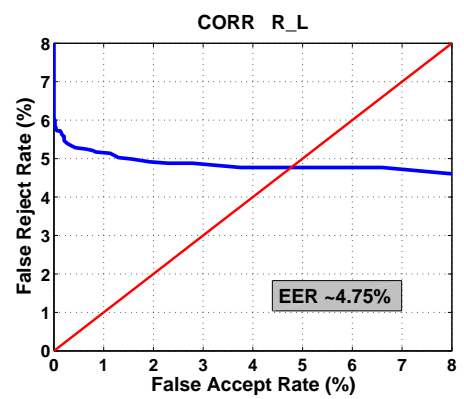

(e)

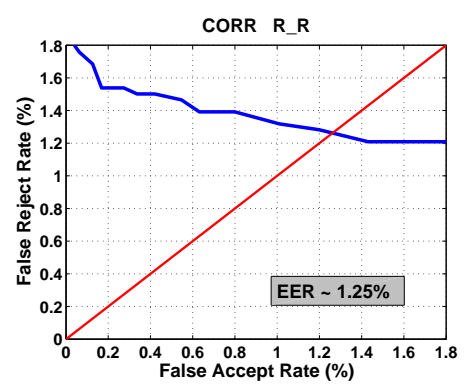

(g)

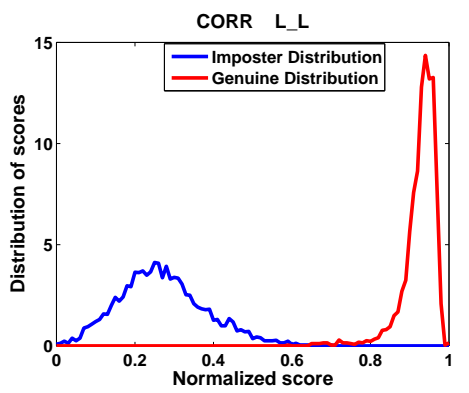

(b)

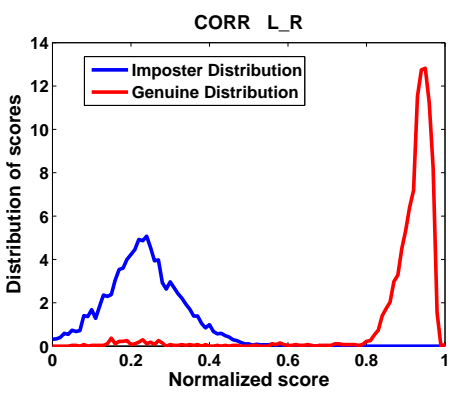

(d)

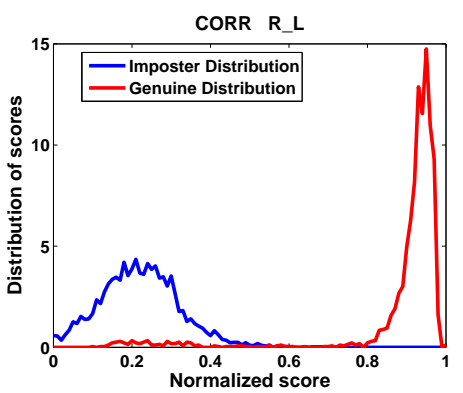

(f)

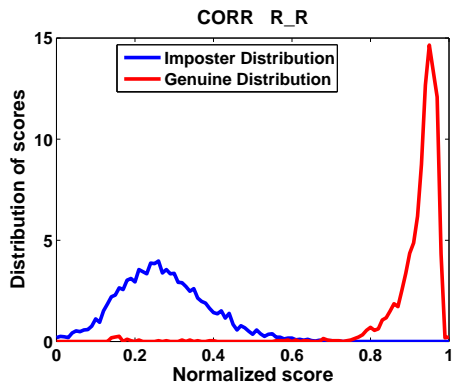

(h)

Figure A.3 Data collection 1. The ROC and the distribution of scores for the correlation technique. 
Chapter A Methods for sclera patterns matching. The ROC and the distribution of scores. Data Collection 1
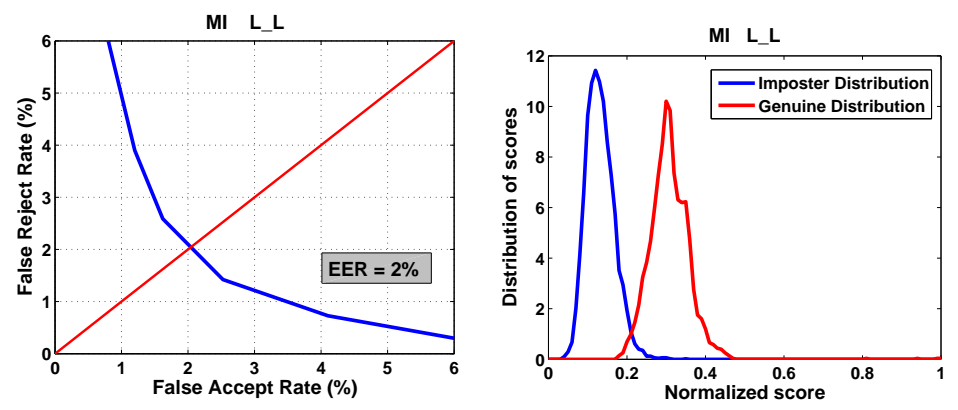

(a)

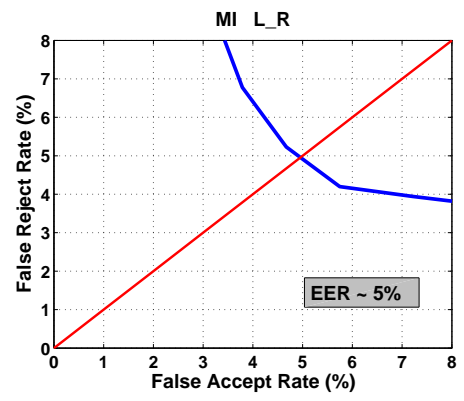

(b)

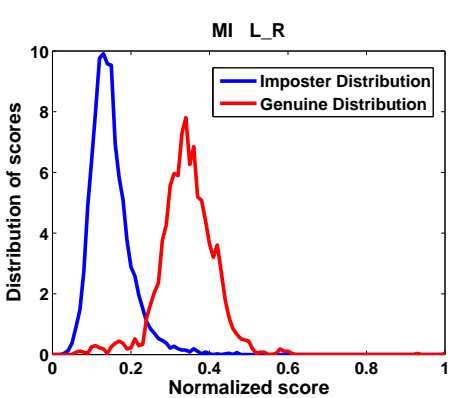

(c)

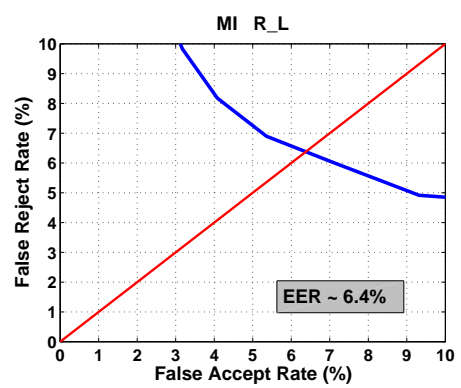

(d)

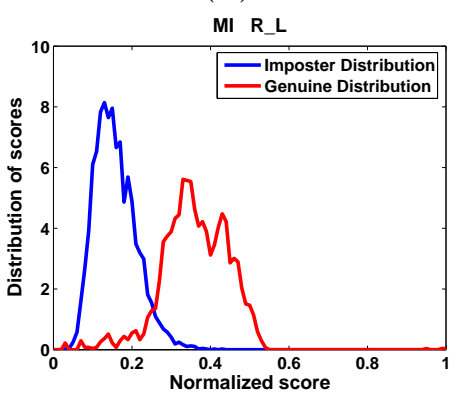

(e)

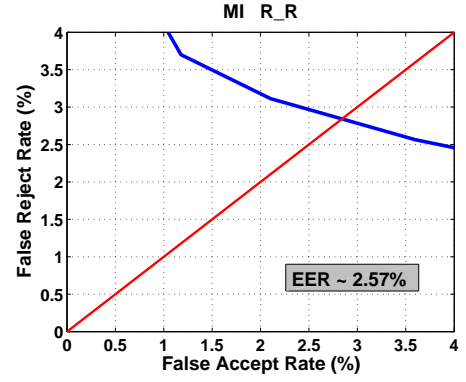

(f)

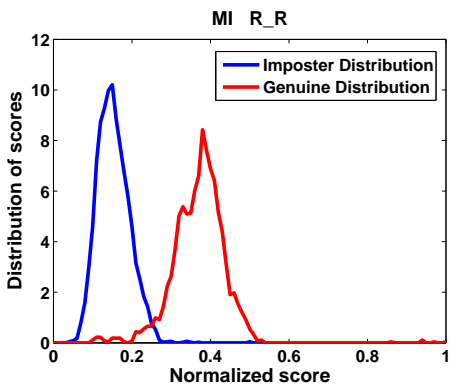

(g)

(h)

Figure A.4 Data collection 1. The ROC and the distribution of scores for the mutual information technique. 
Chapter A Methods for sclera patterns matching. The ROC and the distribution of scores. Data Collection 1

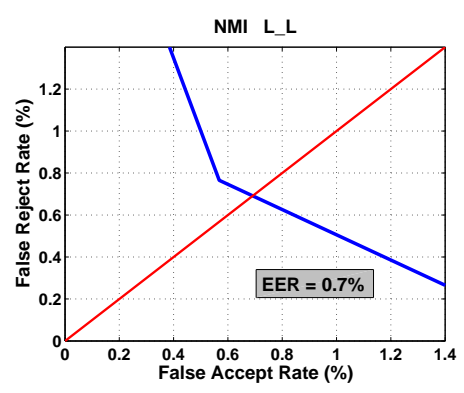

(a)

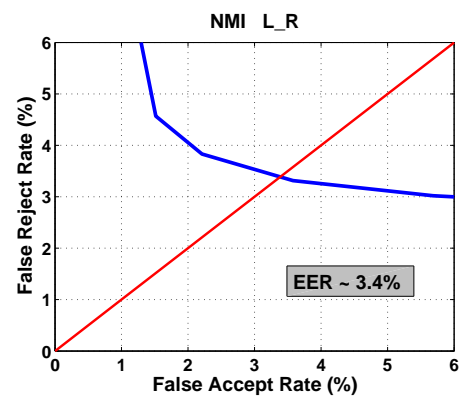

(c)

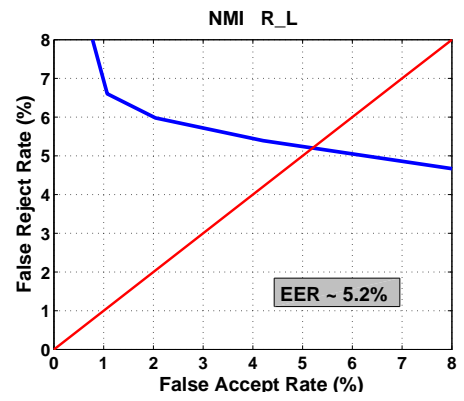

(e)

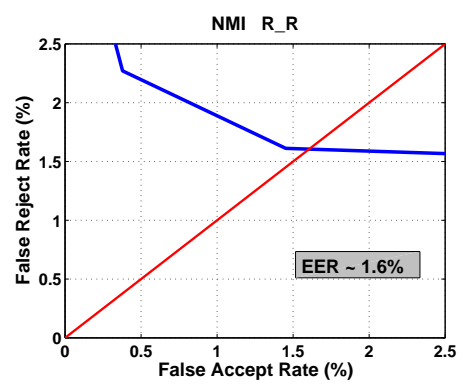

(g)

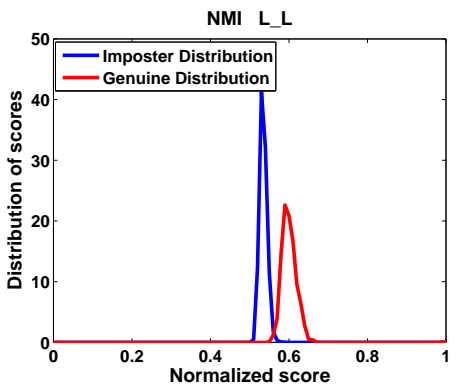

(b)

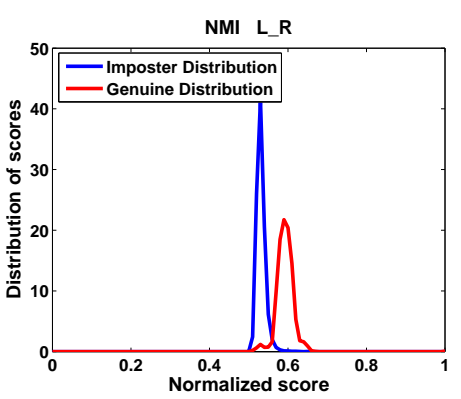

(d)

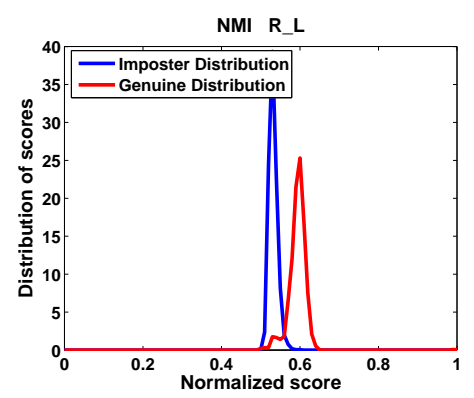

(f)

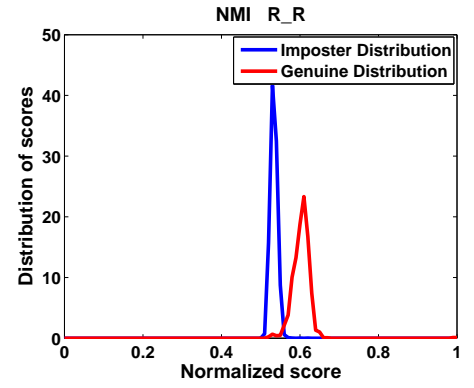

(h)

Figure A.5 Data collection 1. The ROC and the distribution of scores for the normalized mutual information technique. 
Chapter A Methods for sclera patterns matching. The ROC and the distribution of scores. Data Collection 1
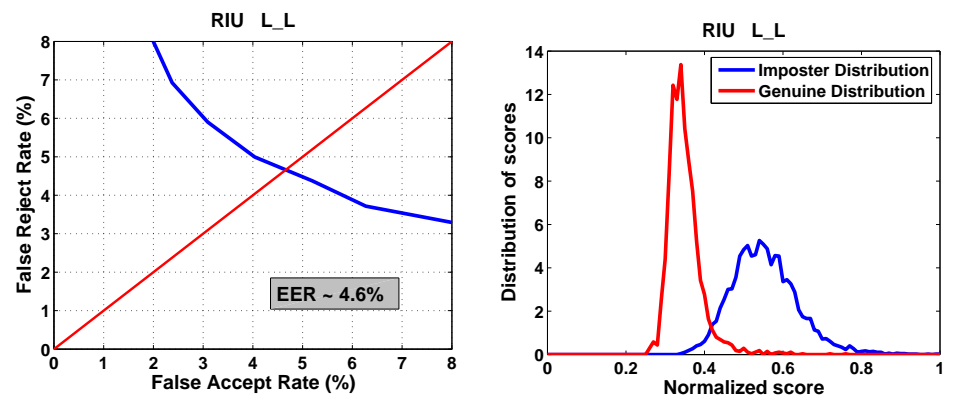

(a)

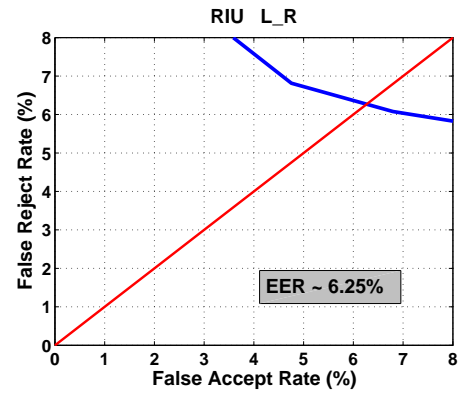

(b)

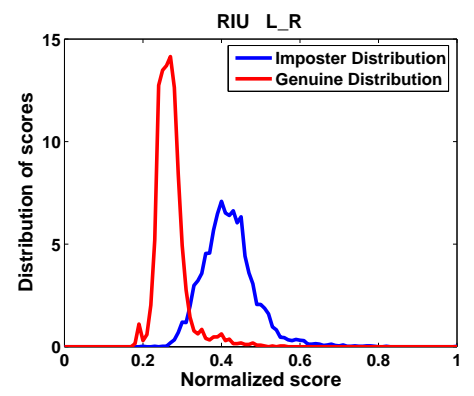

(c)

(d)
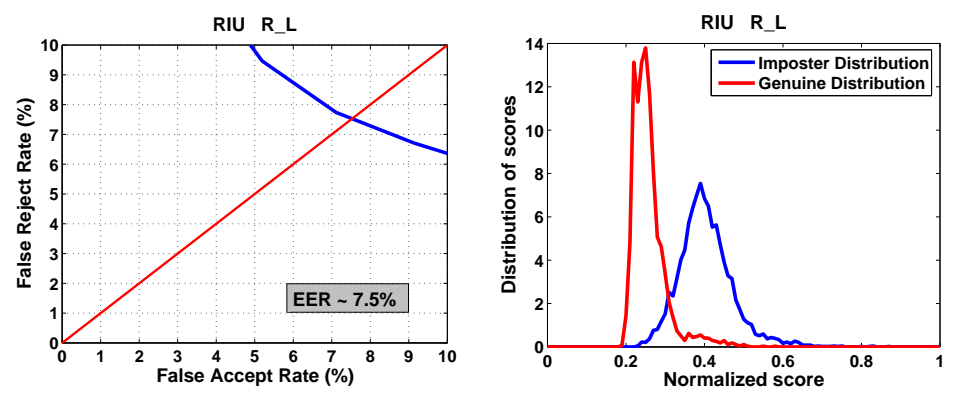

(e)

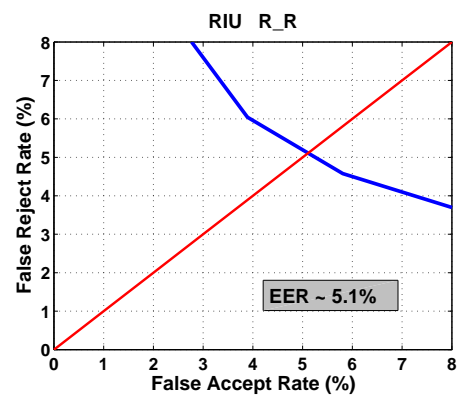

(f)

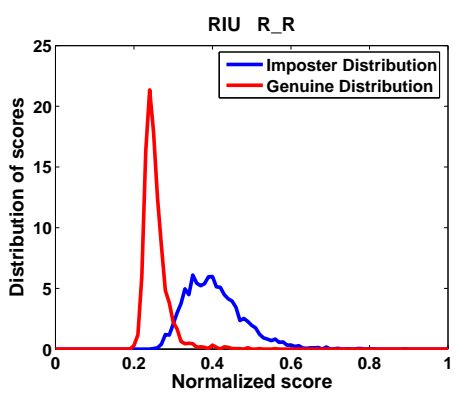

(g)

(h)

Figure A.6 Data collection 1. The ROC and the distribution of scores for the ratio-image uniformity technique. 
Chapter A Methods for sclera patterns matching. The ROC and the distribution of scores. Data Collection 1

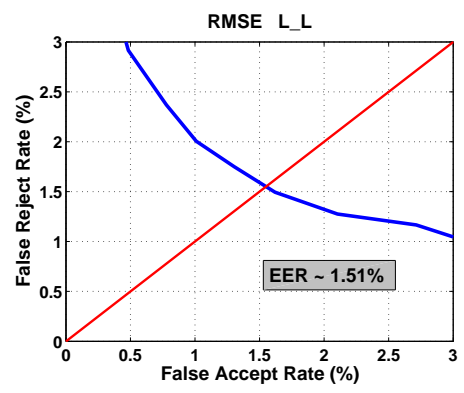

(a)

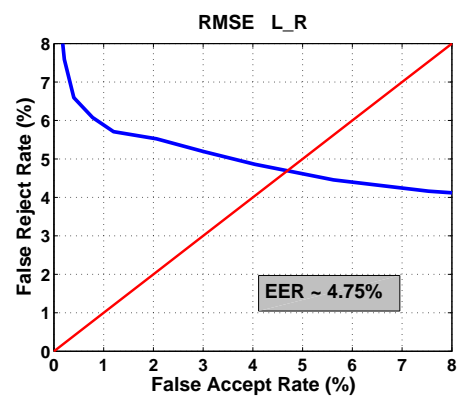

(c)

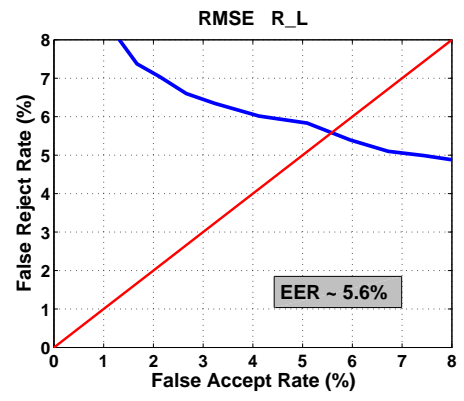

(e)

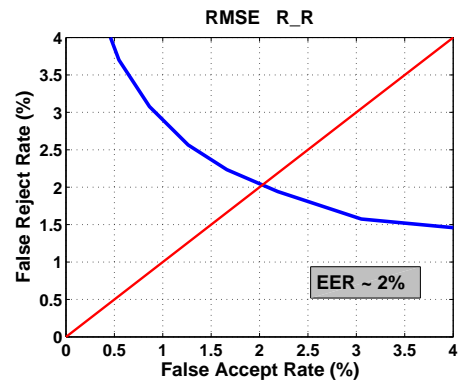

$(\mathrm{g})$

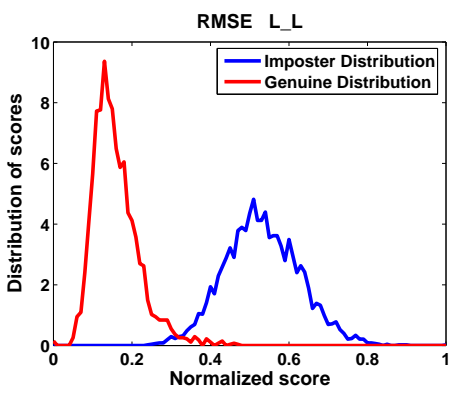

(b)

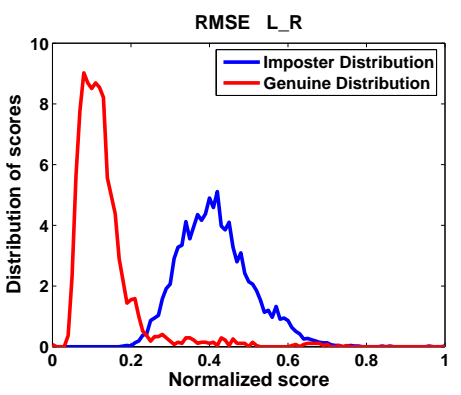

(d)

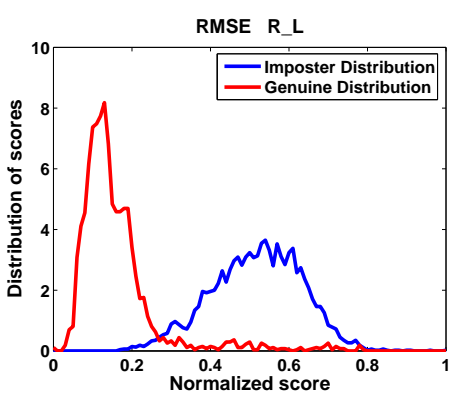

(f)

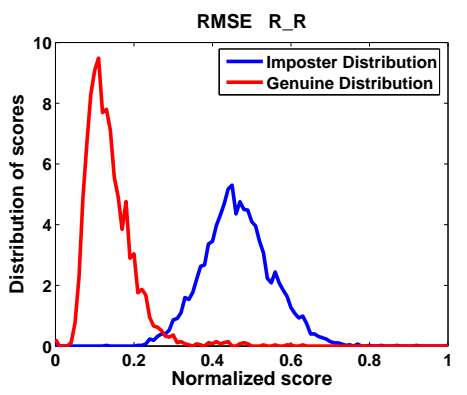

(h)

Figure A.7 Data collection 1. The ROC and the distribution of scores for the root mean square error technique. 
Chapter A Methods for sclera patterns matching. The ROC and the distribution of scores. Data Collection 1
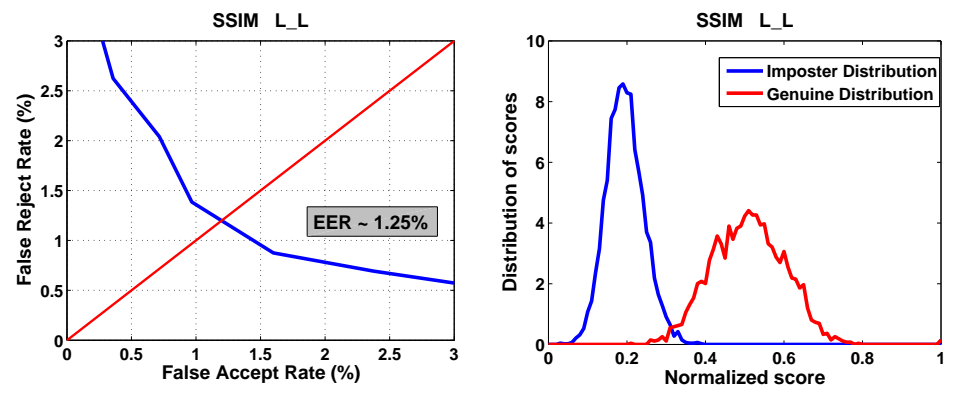

(a)

(b)
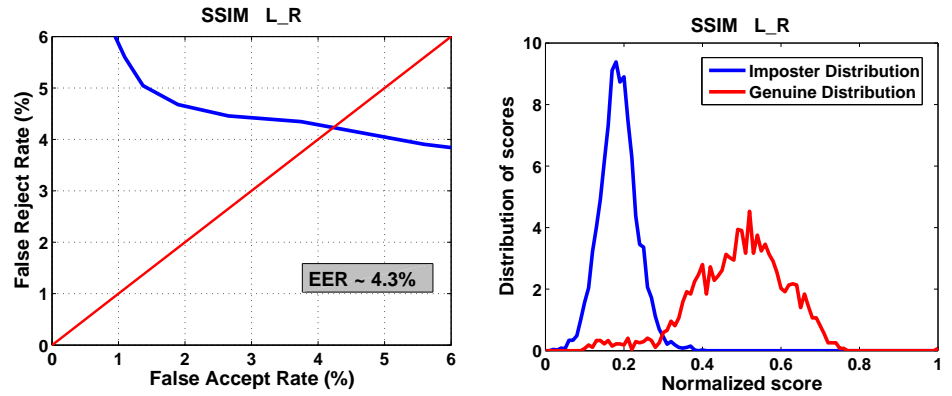

(c)

(d)
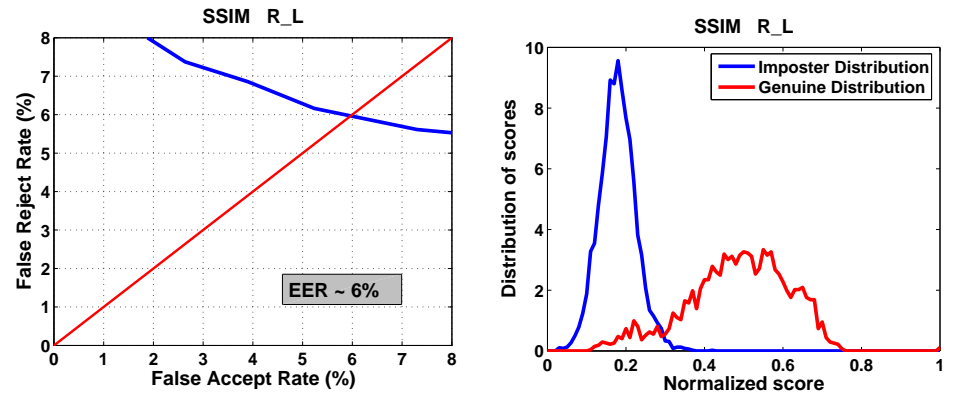

(e)

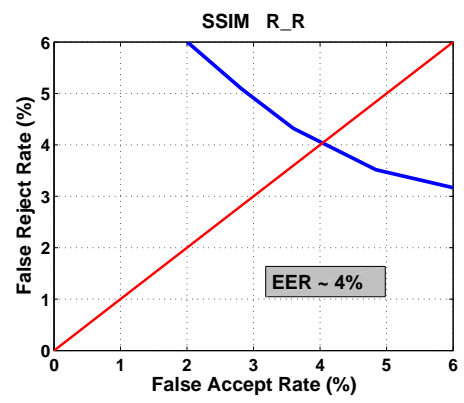

(f)

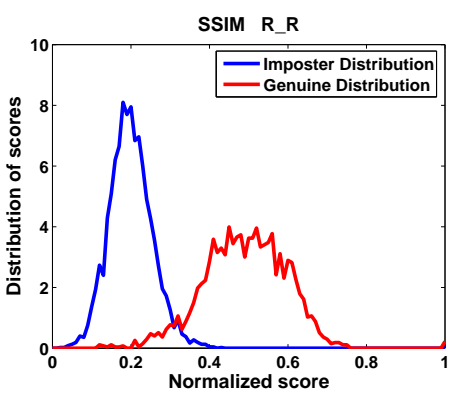

(g)

(h)

Figure A.8 Data collection 1. The ROC and the distribution of scores for the structural similarity index technique. 
Chapter A Methods for sclera patterns matching. The ROC and the distribution of scores. Data Collection 1

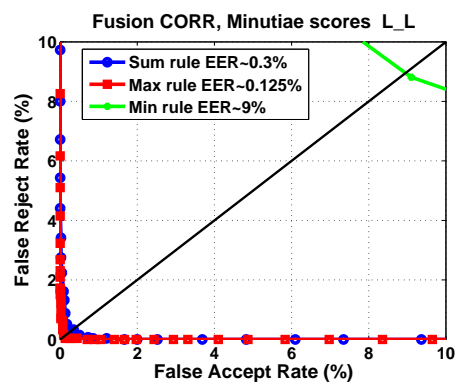

(a)

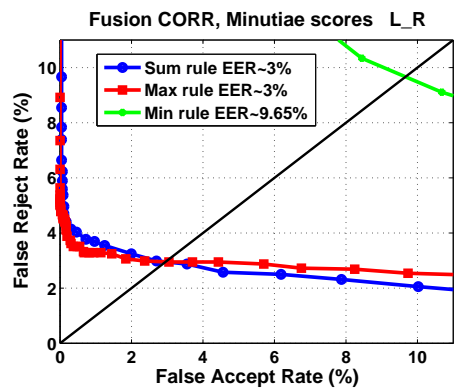

(c)

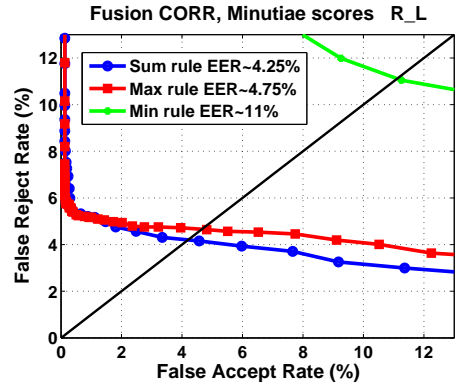

(e)

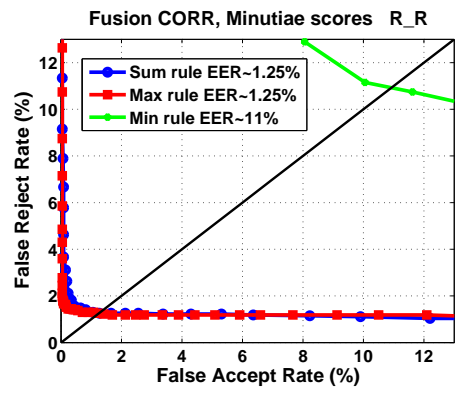

(g)

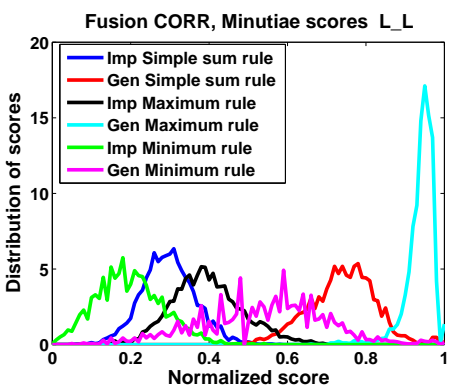

(b)

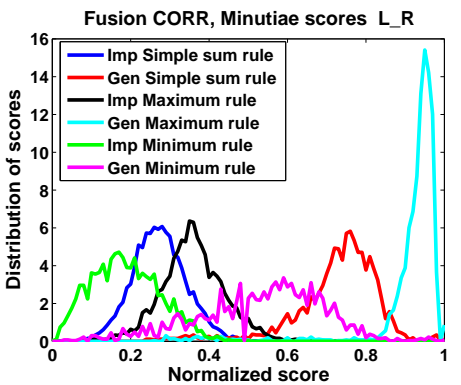

(d)

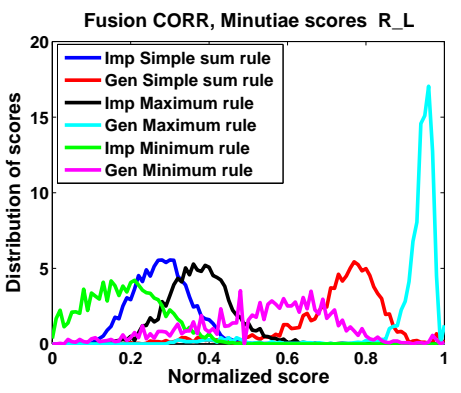

(f)

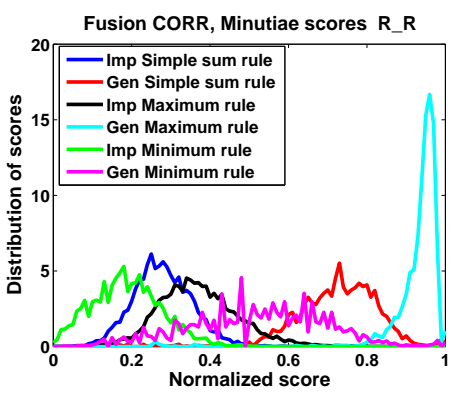

(h)

Figure A.9 Data collection 1. The ROC and the distribution of scores for the fusion of minutiae and correlation technique. 
Chapter A Methods for sclera patterns matching. The ROC and the distribution of scores. Data Collection 1
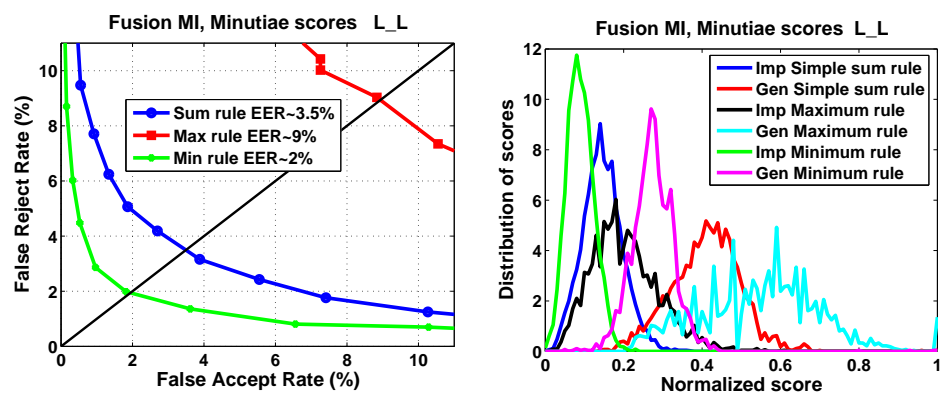

(a)

(b)
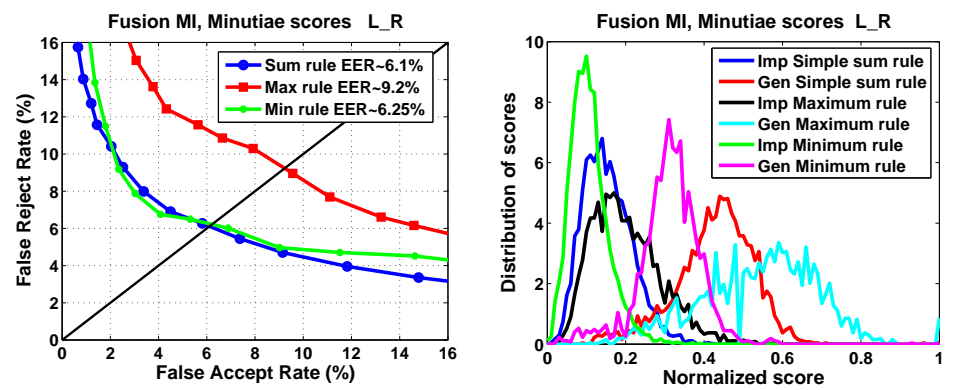

(c)

(d)
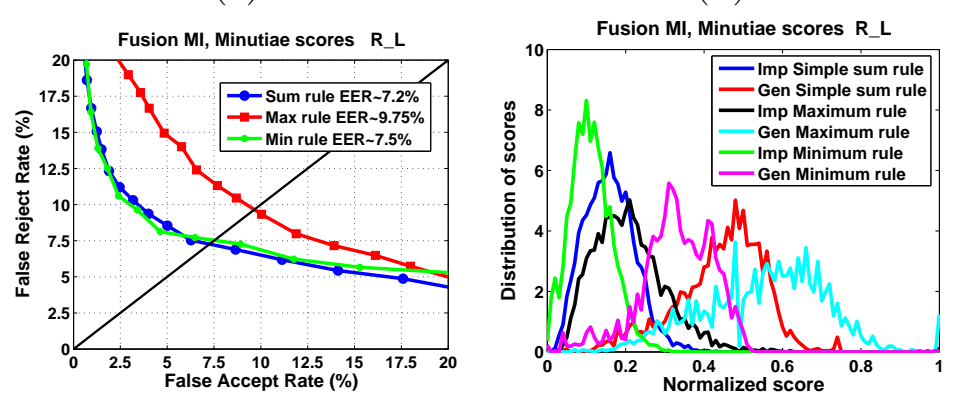

(e)

(f)

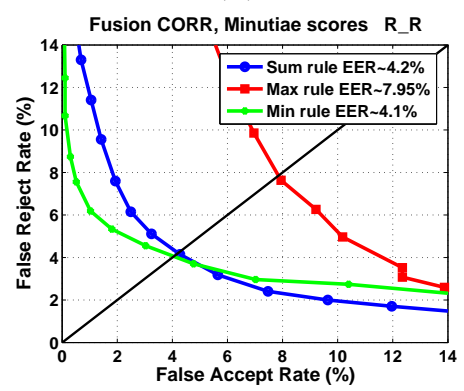

(g)

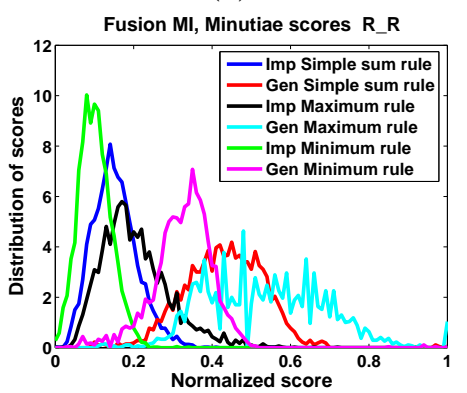

(h)

Figure A.10 Data collection 1. The ROC and the distribution of scores for the fusion of minutiae and mutual information technique. 
Chapter A Methods for sclera patterns matching. The ROC and the distribution of scores. Data Collection 1

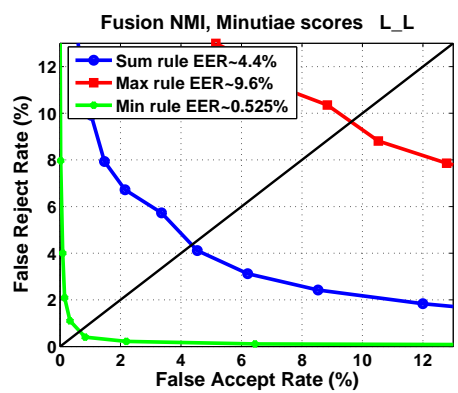

(a)

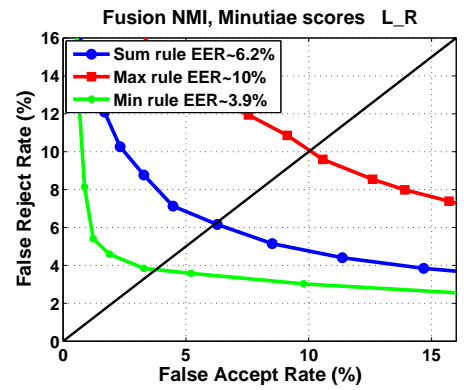

(c)

Fusion NMI, Minutiae scores R_L

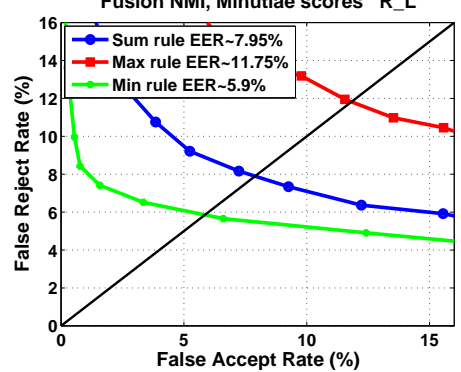

(e)

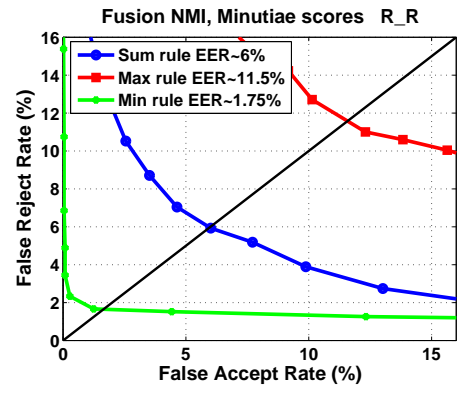

(g)

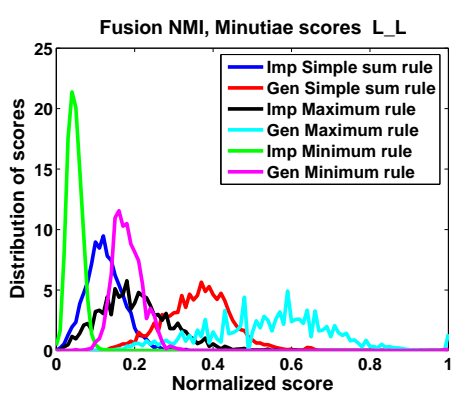

(b)

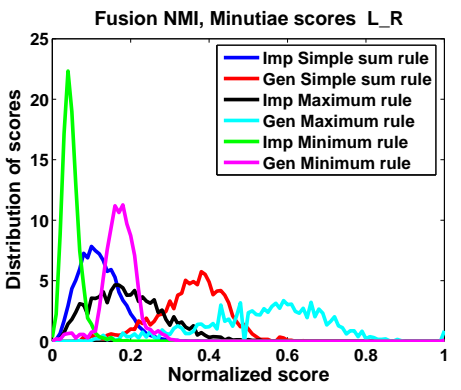

(d)

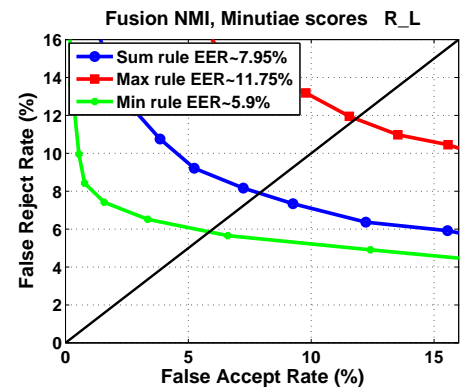

(f)

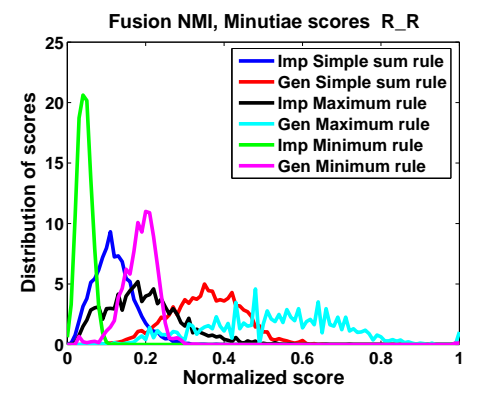

(h)

Figure A.11 Data collection 1. The ROC and the distribution of scores for the fusion of minutiae and normalized mutual information technique. 


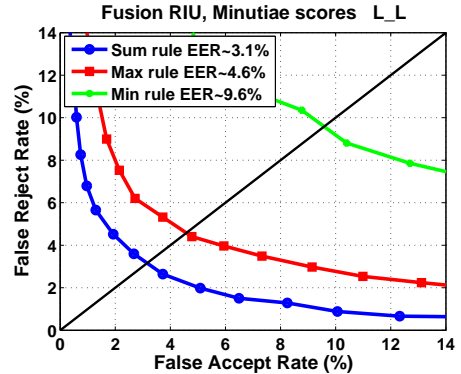

(a)

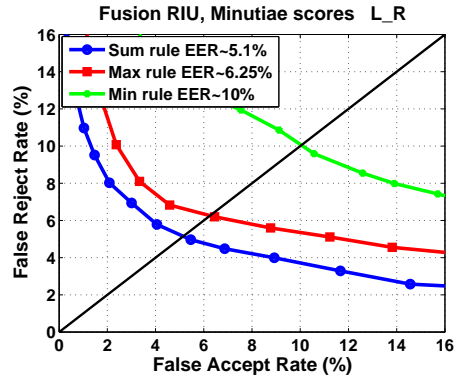

(c)

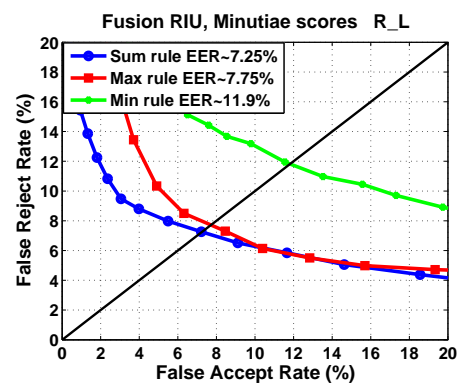

(e)

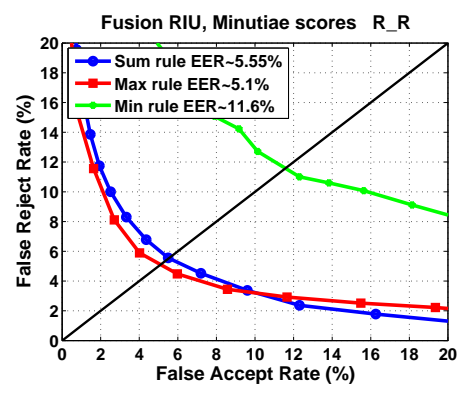

$(g)$

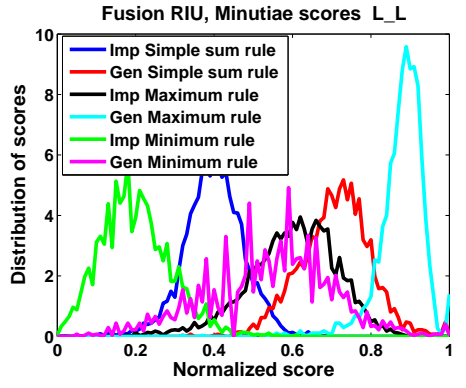

(b)

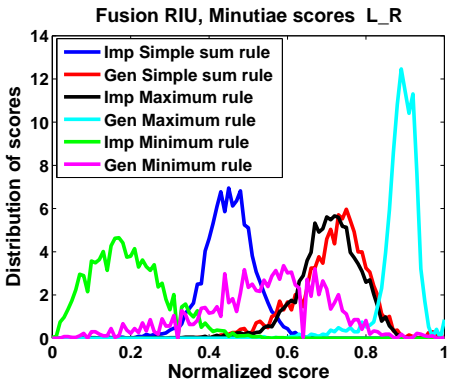

(d)

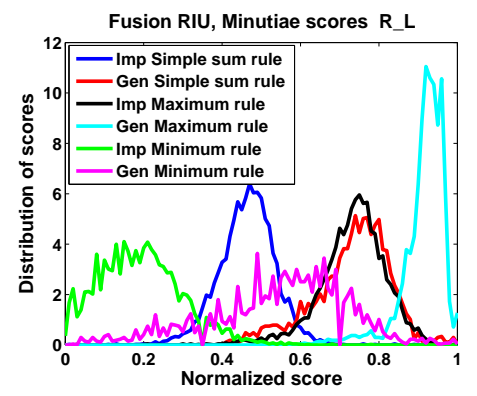

(f)

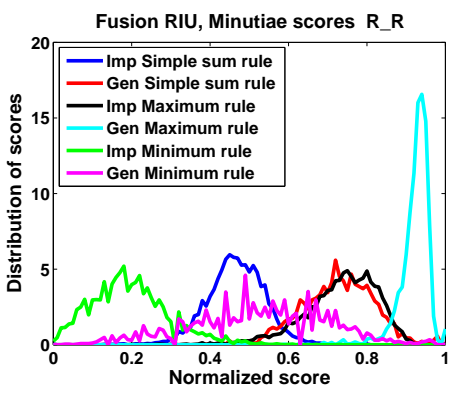

(h)

Figure A.12 Data collection 1. The ROC and the distribution of scores for the fusion of minutiae and ratio-image uniformity technique. 
Chapter A Methods for sclera patterns matching. The ROC and the distribution of scores. Data Collection 1

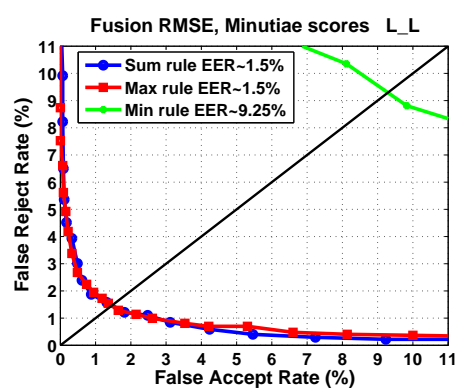

(a)

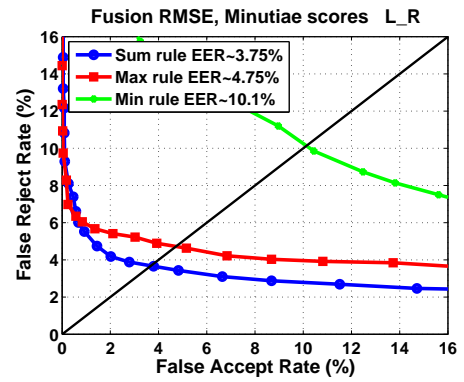

(c)

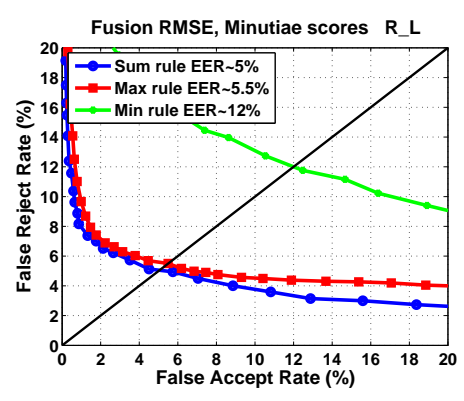

(e)

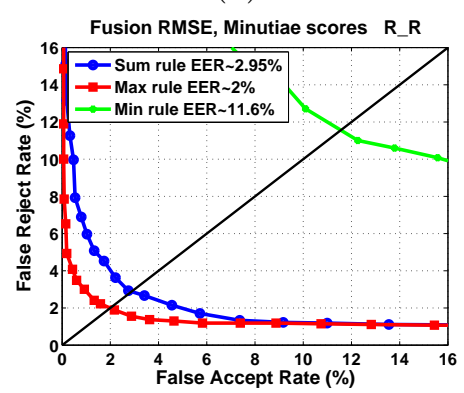

(g)

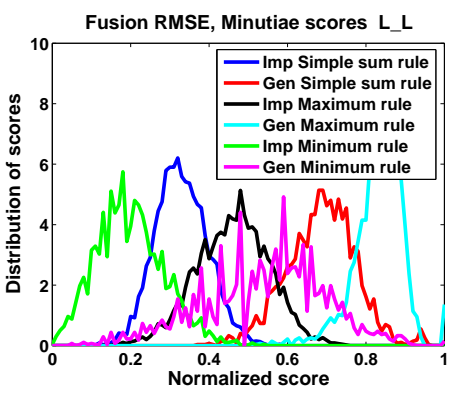

(b)

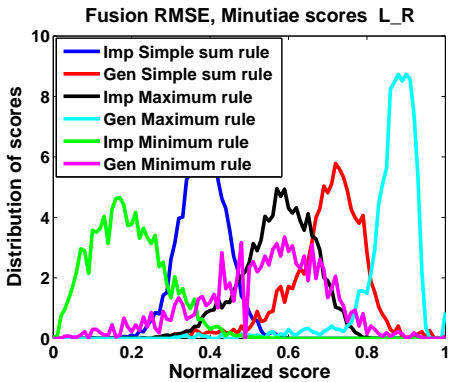

(d)

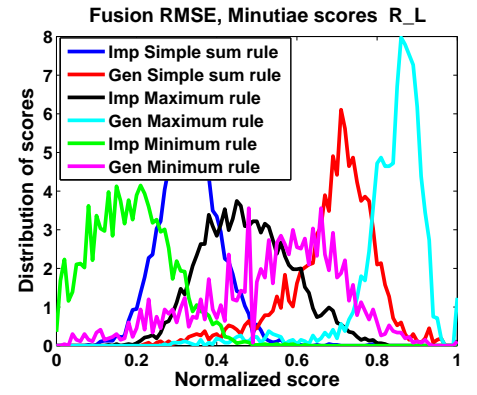

(f)

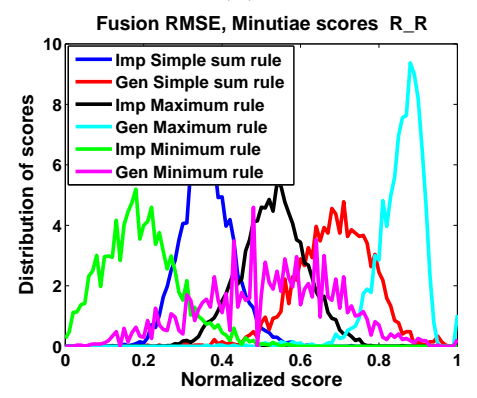

(h)

Figure A.13 Data collection 1. The ROC and the distribution of scores for the fusion of minutiae and root mean square error technique. 
Chapter A Methods for sclera patterns matching. The ROC and the distribution of scores. Data Collection 1

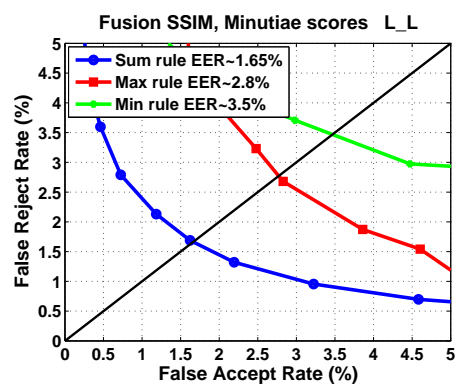

(a)

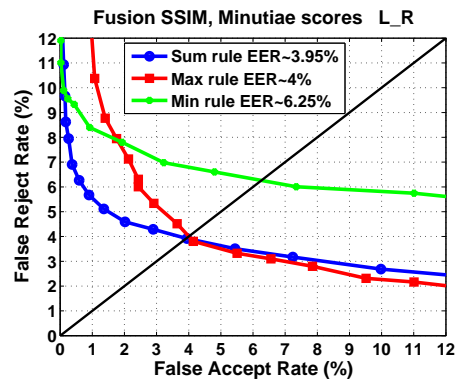

(c)

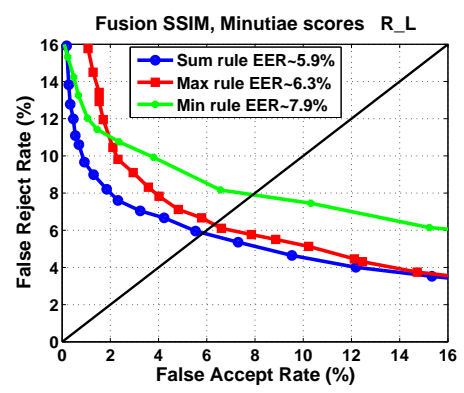

(e)

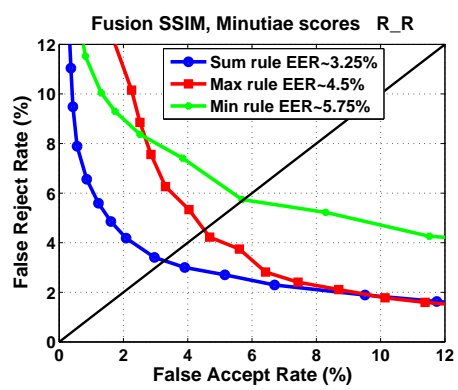

(g)

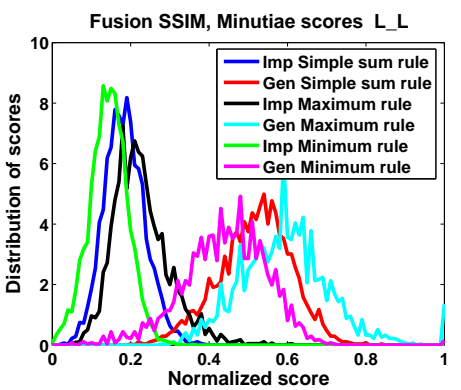

(b)

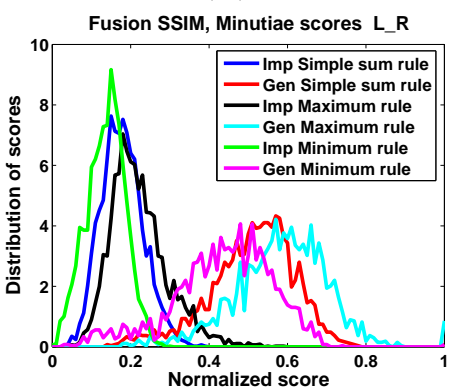

(d)

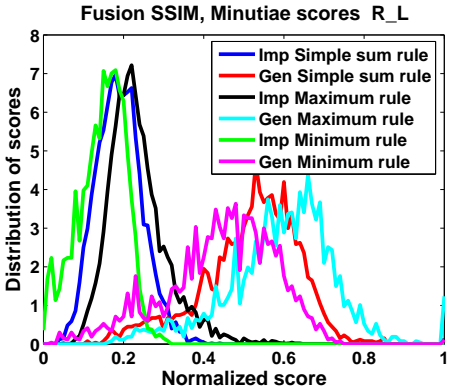

(f)

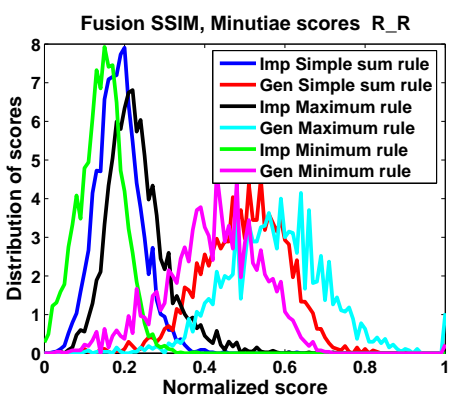

(h)

Figure A.14 Data collection 1. The ROC and the distribution of scores for the fusion of minutiae and structural similarity index technique. 
Appendix B

Fusion of iris patterns with scleral

patterns. The ROC and the

distribution of scores. Data

Collection 1 
Chapter B Fusion of iris patterns with scleral patterns. The ROC and the distribution of scores. Data Collection 1

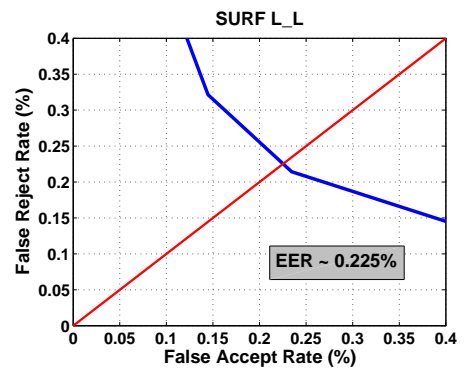

(a)

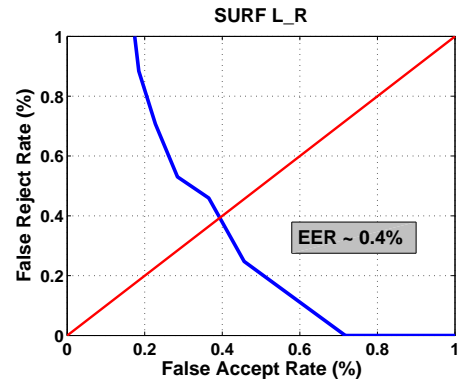

(c)

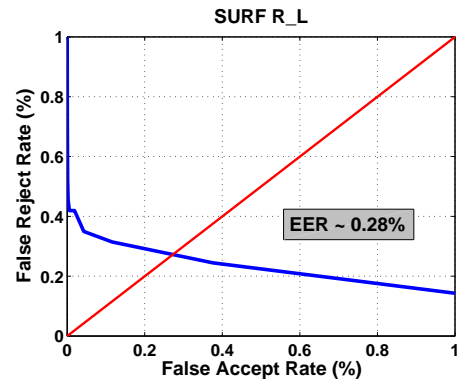

(e)

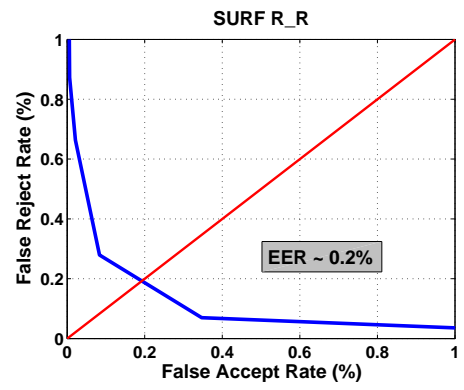

(g)

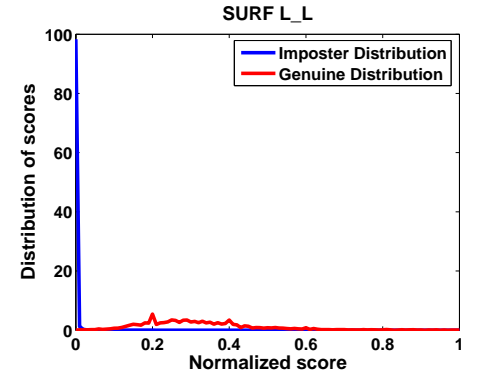

(b)

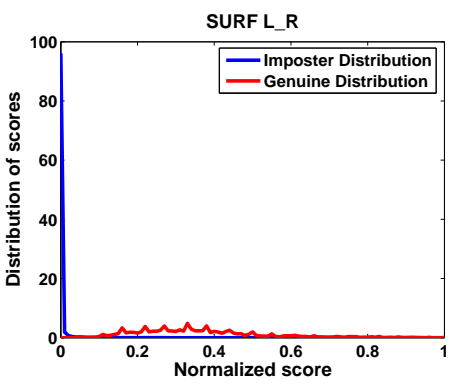

(d)

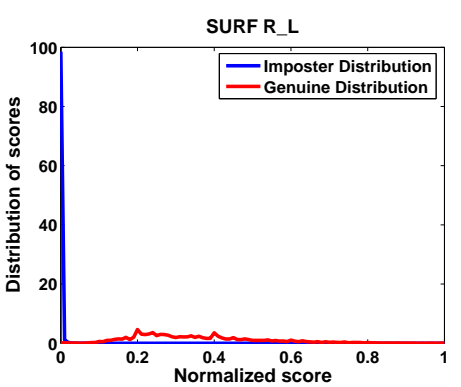

(f)

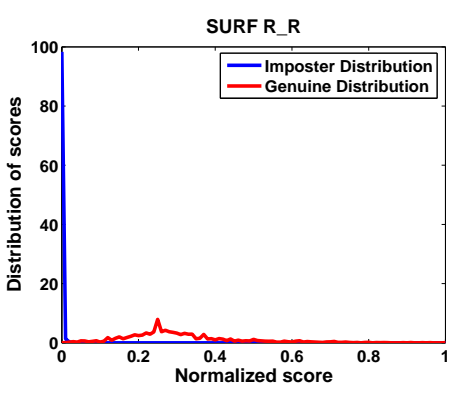

(h)

Figure B.1 Data collection 1. The ROC and the distribution of scores for the SURF technique (automatic sclera segmentation). 
Chapter B Fusion of iris patterns with scleral patterns. The ROC and the distribution of scores. Data Collection 1

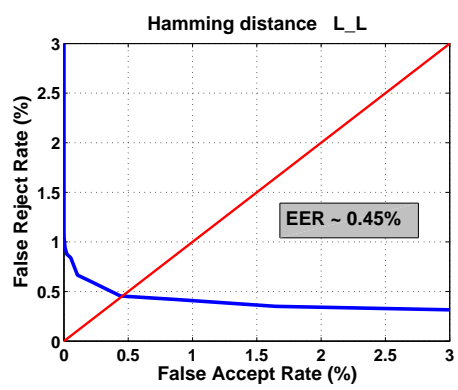

(a)

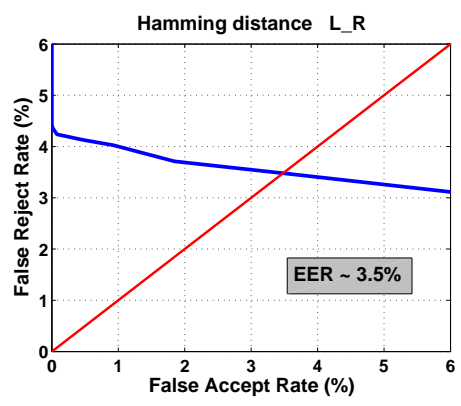

(c)

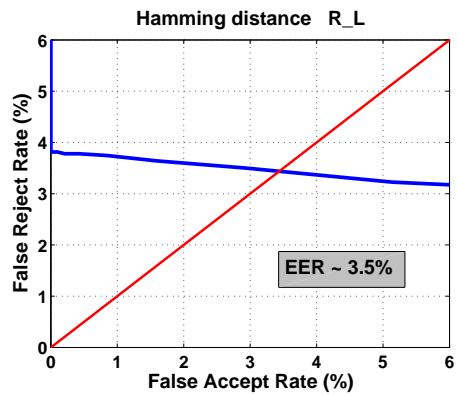

(e)

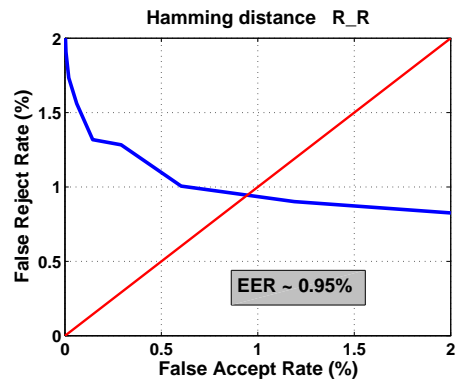

(g)

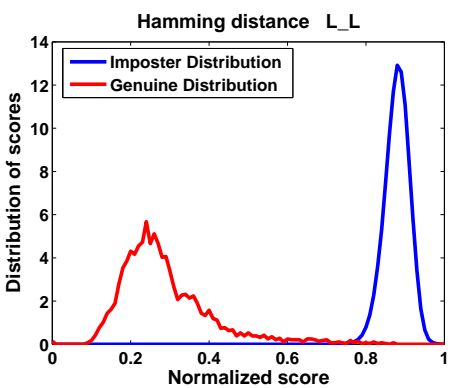

(b)

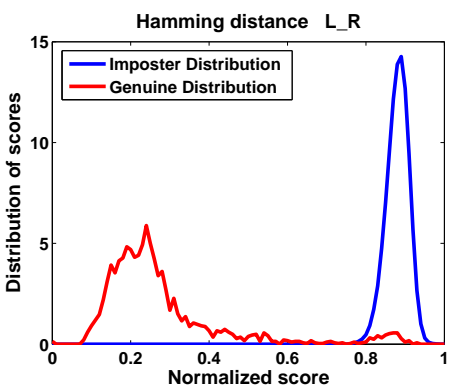

(d)

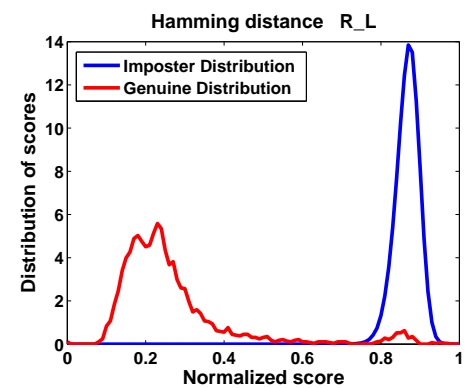

(f)

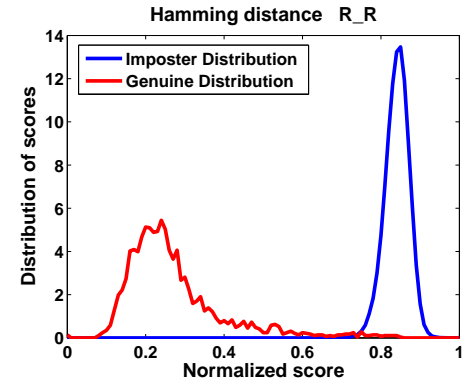

(h)

Figure B.2 Data collection 1. The ROC and the distribution of scores for the Hamming distance. 
Chapter B Fusion of iris patterns with scleral patterns. The ROC and the distribution of scores. Data Collection 1

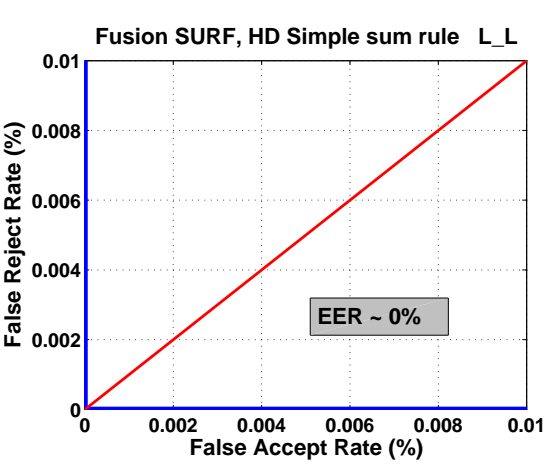

(a)

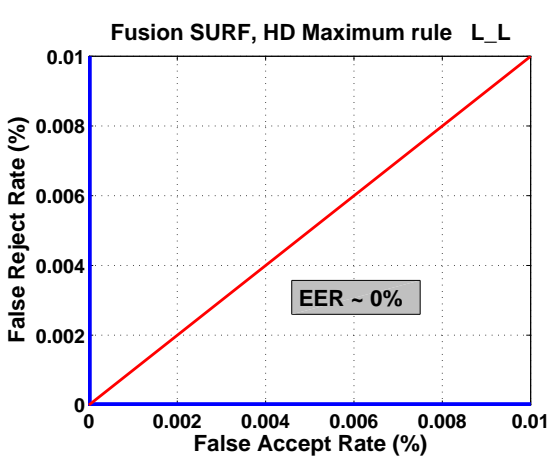

(c)

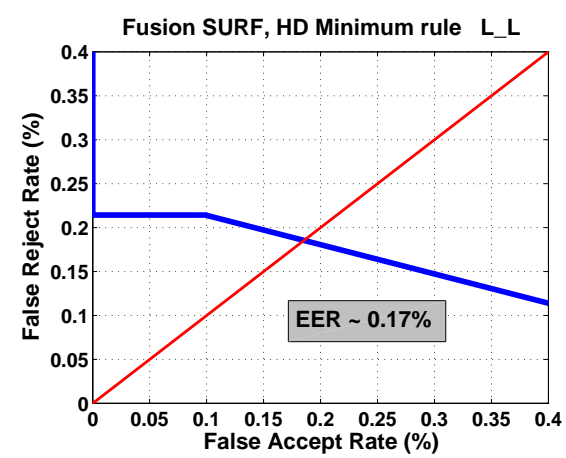

(e)

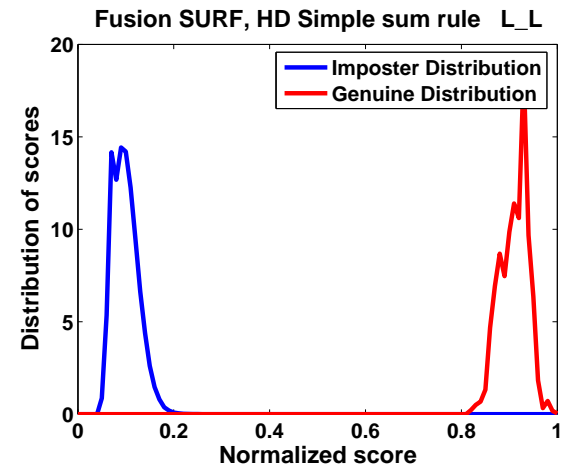

(b)

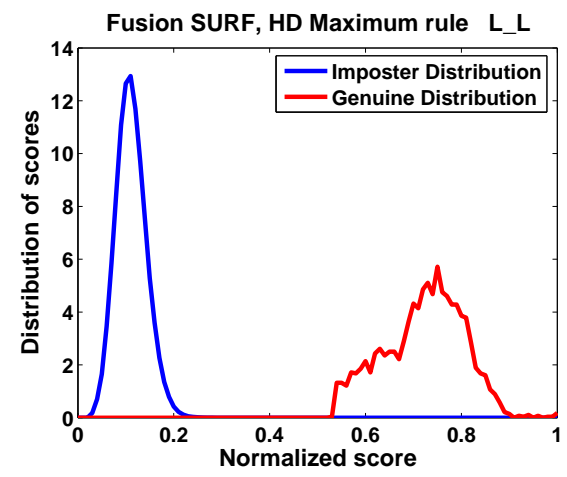

(d)

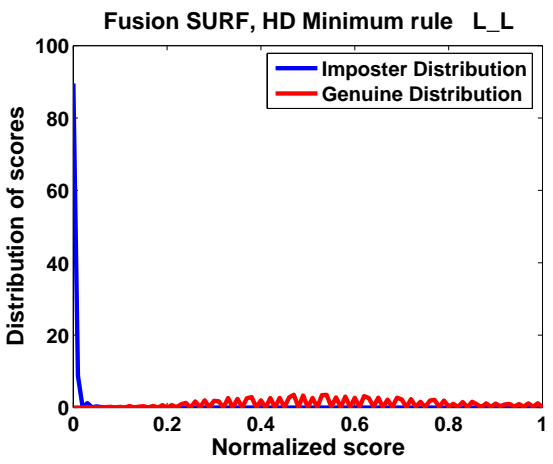

(f)

Figure B.3 Data collection 1. Fusion of iris patterns and sclera patterns. The ROC and the distribution of scores for L_L, simple sum rule, maximum rule, minimum rule. 
Chapter B Fusion of iris patterns with scleral patterns. The ROC and the distribution of scores. Data Collection 1

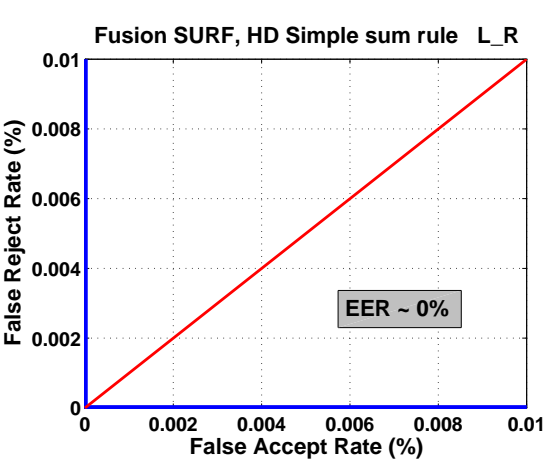

(a)

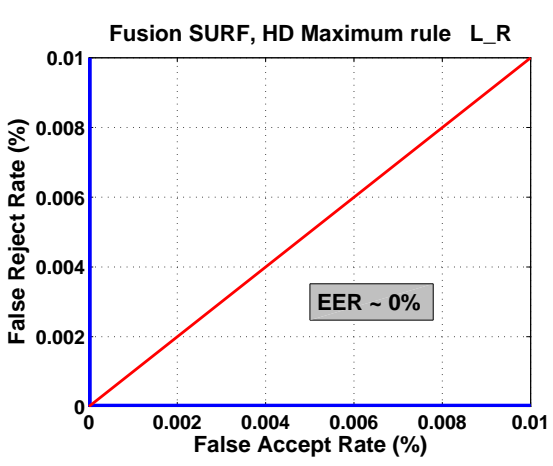

(c)

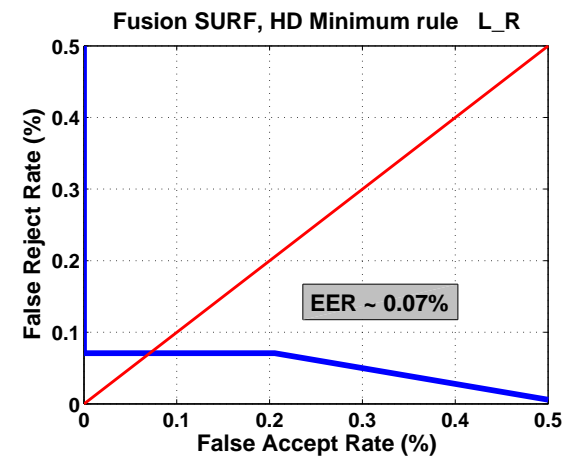

(e)

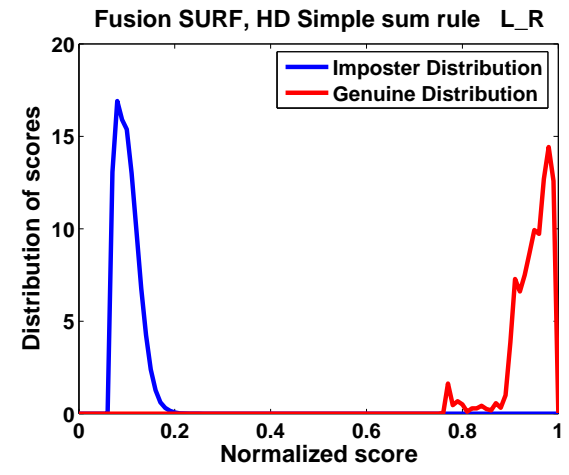

(b)

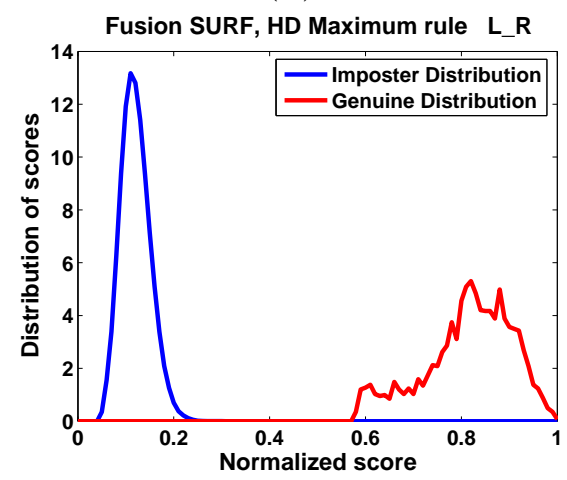

(d)

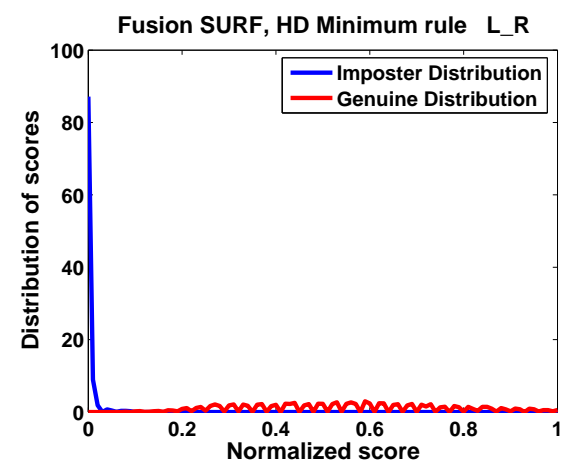

(f)

Figure B.4 Data collection 1. Fusion of iris patterns and sclera patterns. The ROC and the distribution of scores for $L_{-} \mathrm{R}$, simple sum rule, maximum rule, minimum rule. 
Chapter B Fusion of iris patterns with scleral patterns. The ROC and the distribution of scores. Data Collection 1

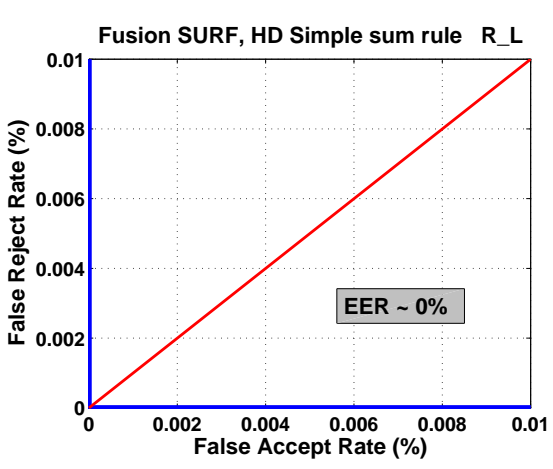

(a)

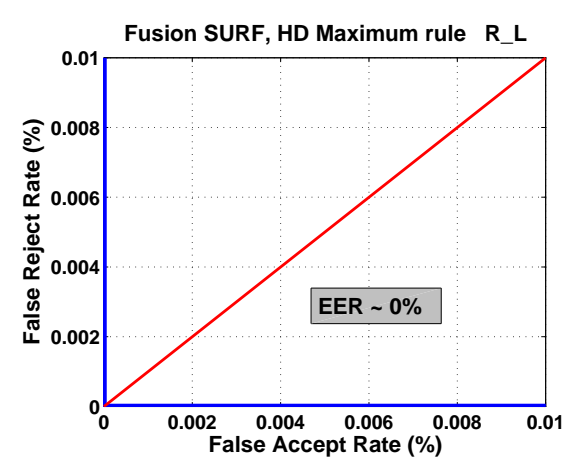

(c)

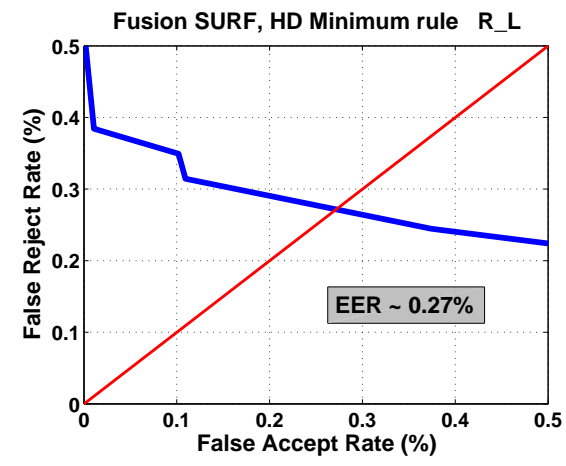

(e)

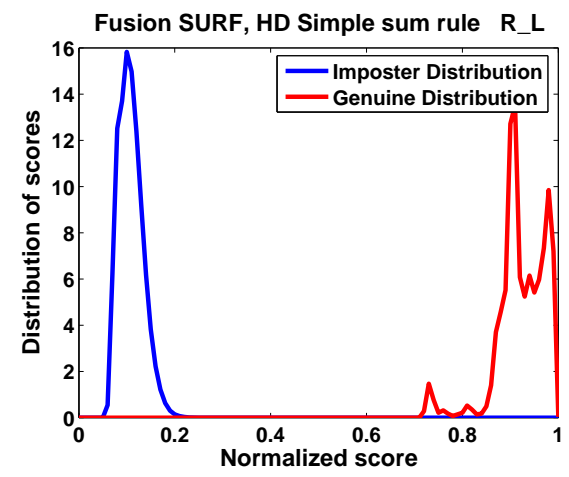

(b)

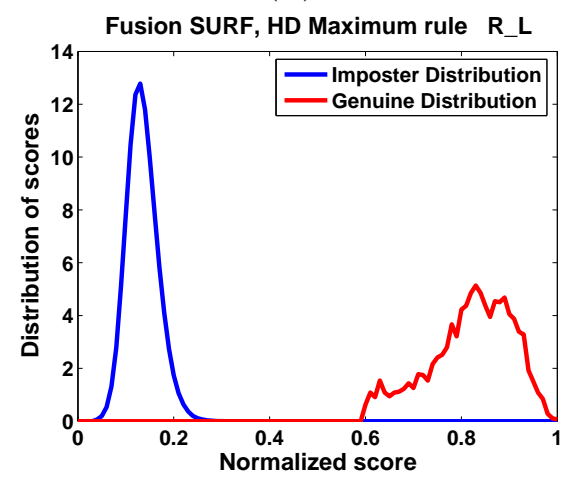

(d)

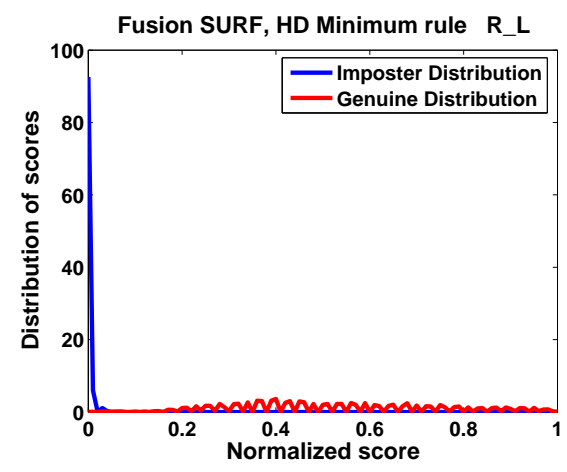

(f)

Figure B.5 Data collection 1. Fusion of iris patterns and sclera patterns. The ROC and the distribution of scores for R_L, simple sum rule, maximum rule, minimum rule. 
Chapter B Fusion of iris patterns with scleral patterns. The ROC and the distribution of scores. Data Collection 1

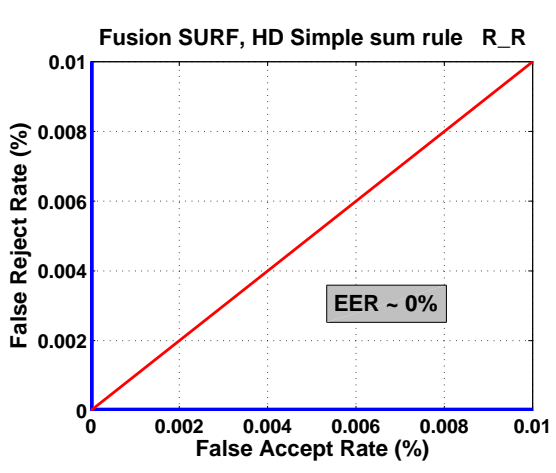

(a)

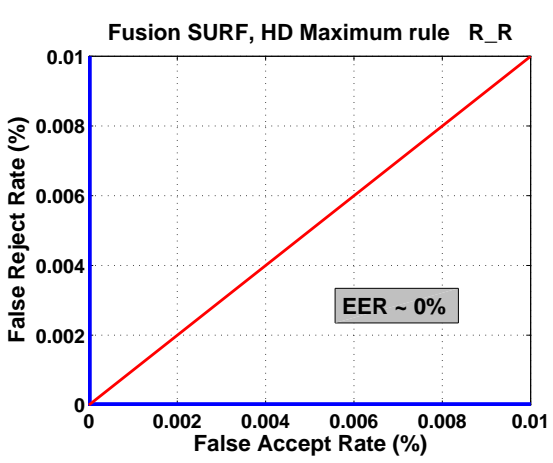

(c)

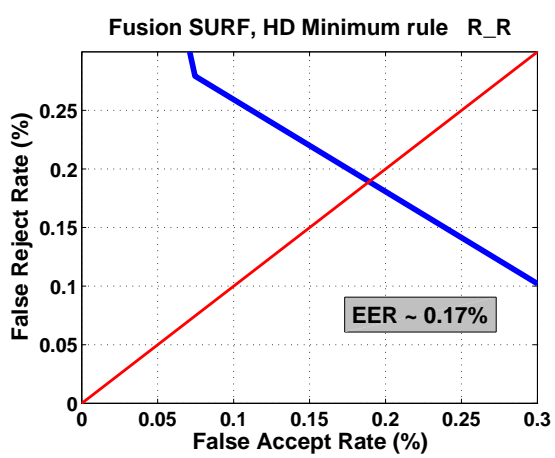

(e)

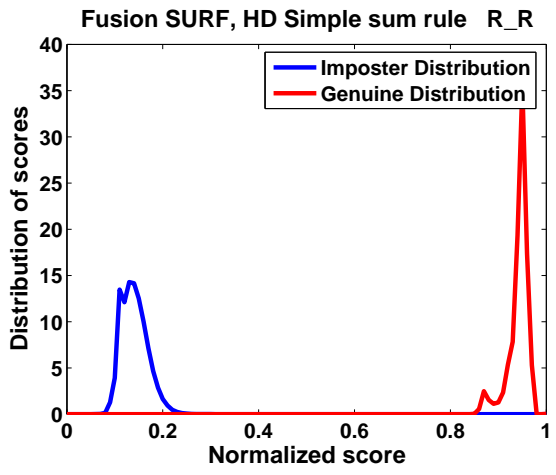

(b)

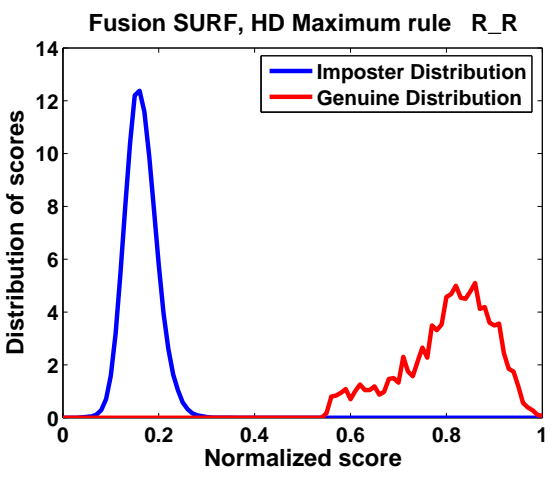

(d)

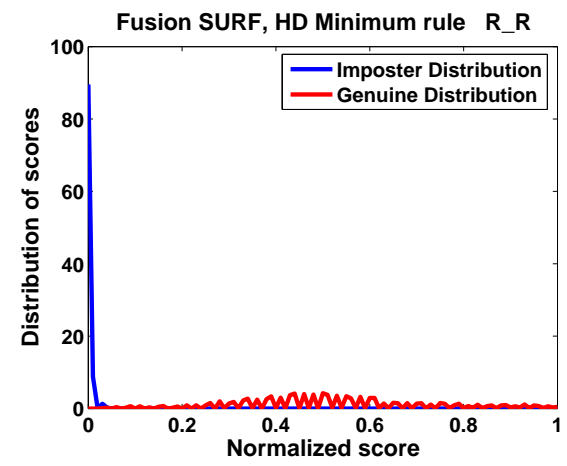

(f)

Figure B.6 Data collection 1. Fusion of iris patterns and sclera patterns. The ROC and the distribution of scores for $R_{-} R$, simple sum rule, maximum rule, minimum rule. 
Appendix C

Impact of intra-class variation.

The ROC and the distribution of scores. Data collection 2. 
Chapter $C$ Impact of intra-class variation. The ROC and the distribution of scores. Data collection 2.

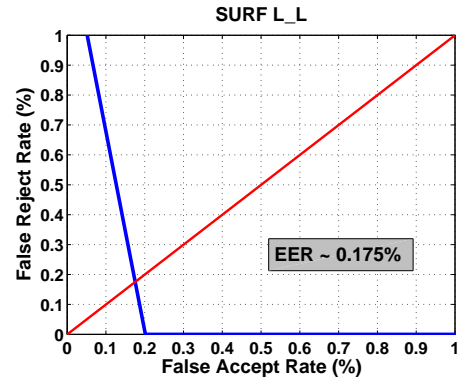

(a)

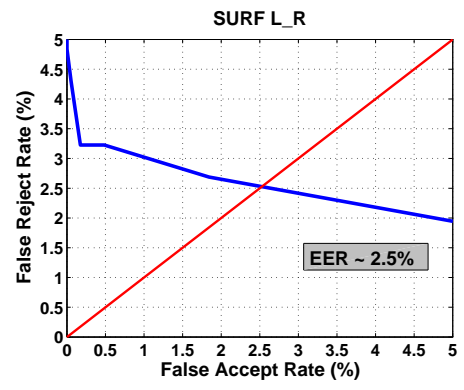

(c)

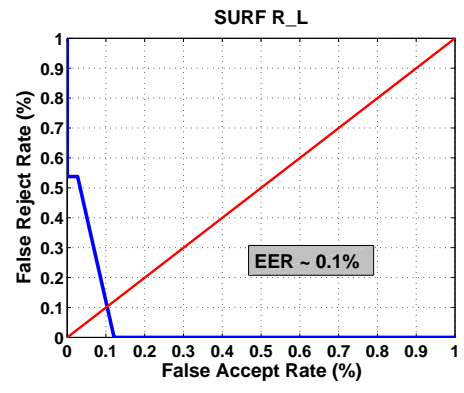

(e)

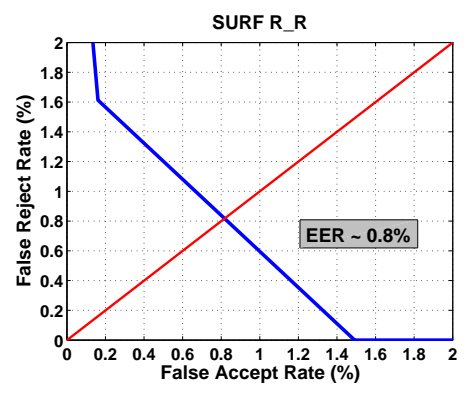

$(g)$

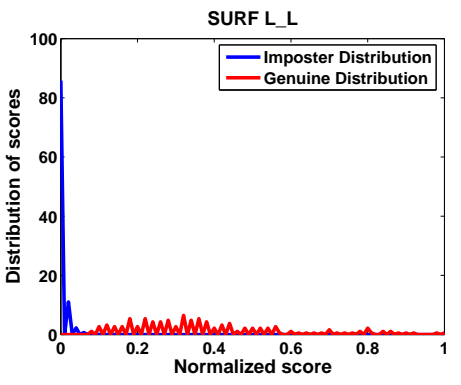

(b)

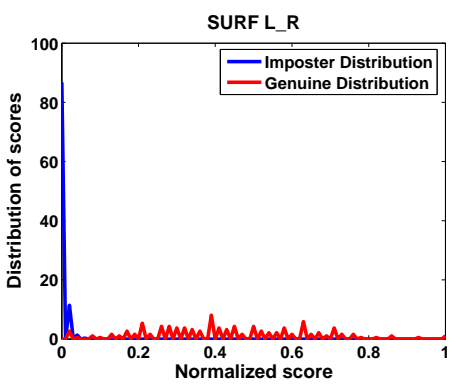

(d)

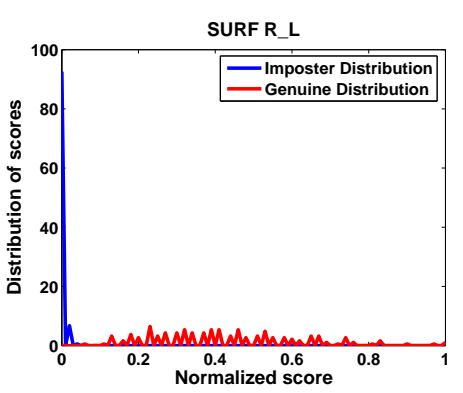

(f)

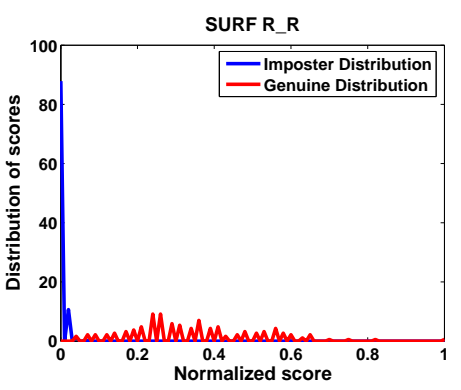

(h)

Figure C.1 Data collection 2. The ROC and the distribution of scores for the SURF technique. 
Chapter $C$ Impact of intra-class variation. The ROC and the distribution of scores. Data collection 2.

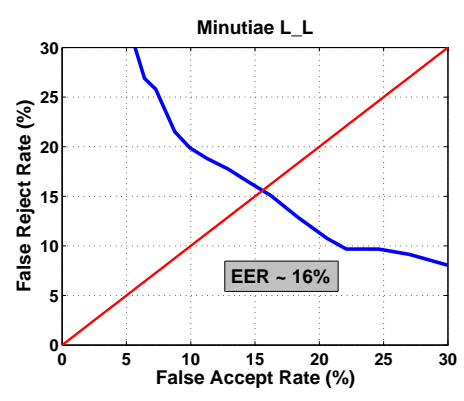

(a)

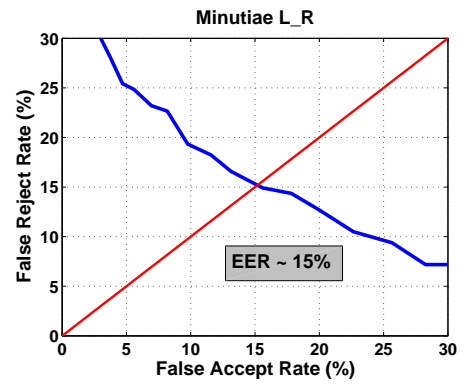

(c)

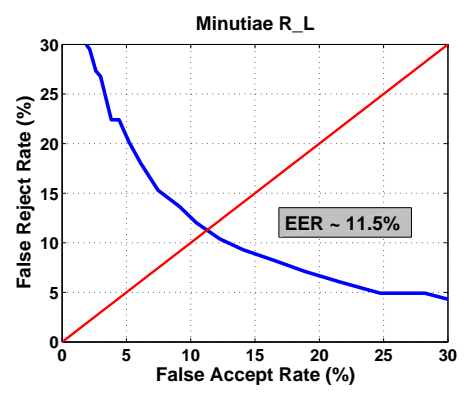

(e)

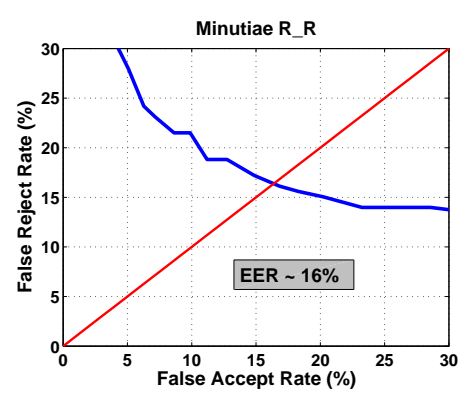

(g)

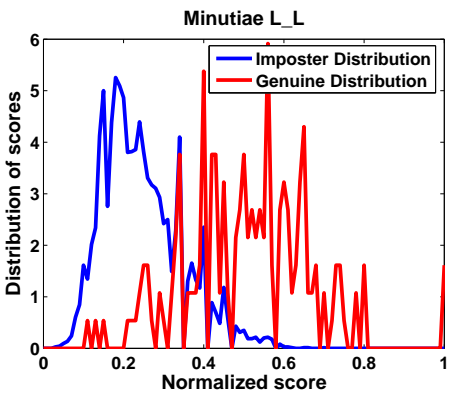

(b)

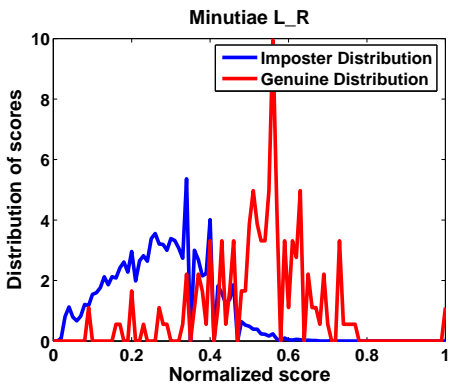

(d)

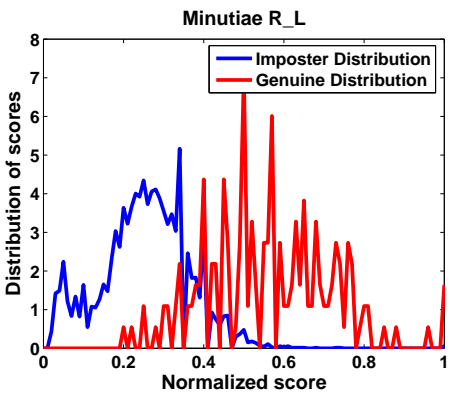

(f)

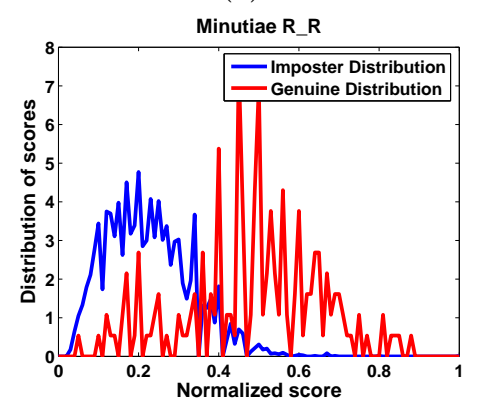

(h)

Figure C.2 Data collection 2. The ROC and the distribution of scores for the minutiae-based matching technique. 
Chapter $C$ Impact of intra-class variation. The ROC and the distribution of scores. Data collection 2.

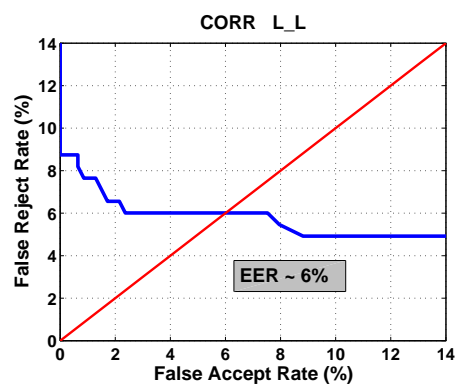

(a)

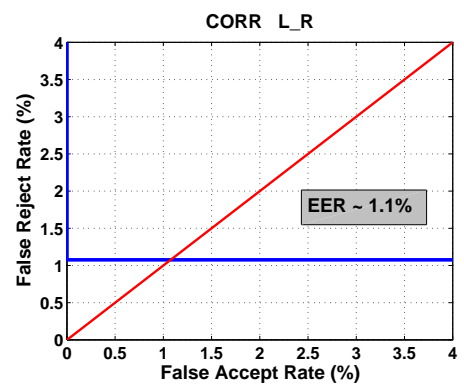

(c)

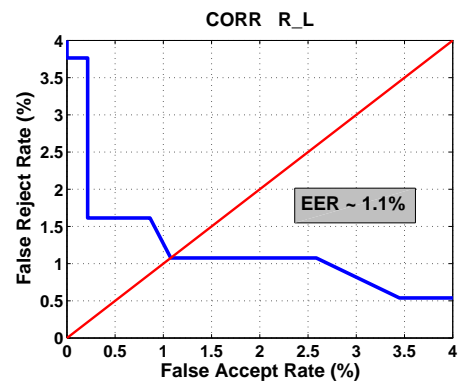

(e)

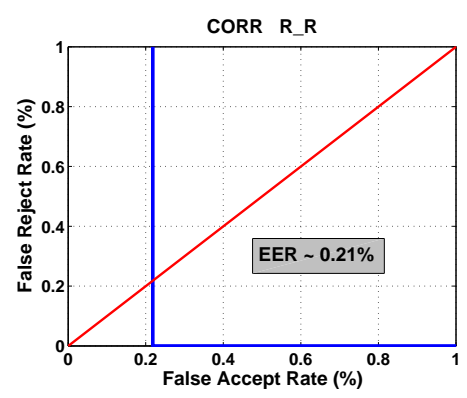

(g)

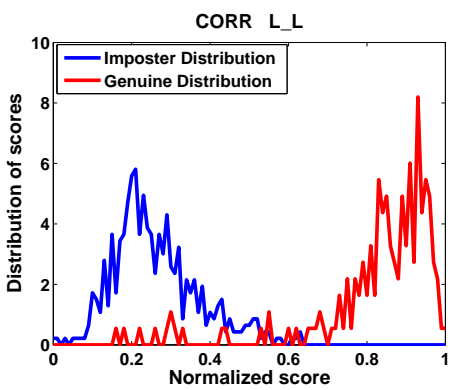

(b)

CORR L_R

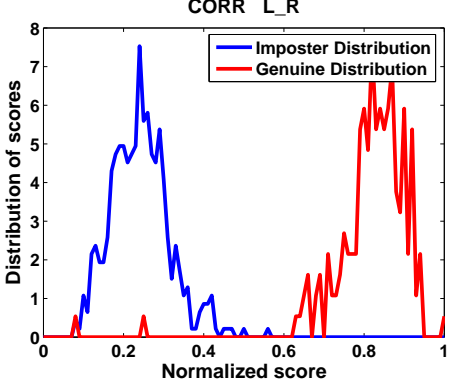

(d)

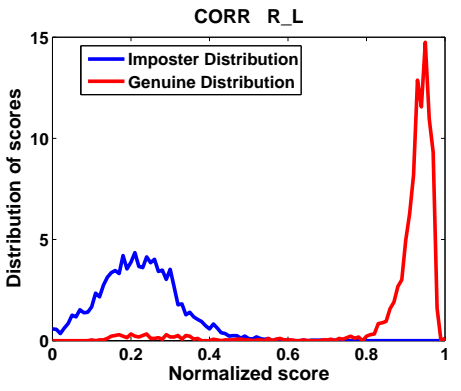

(f)

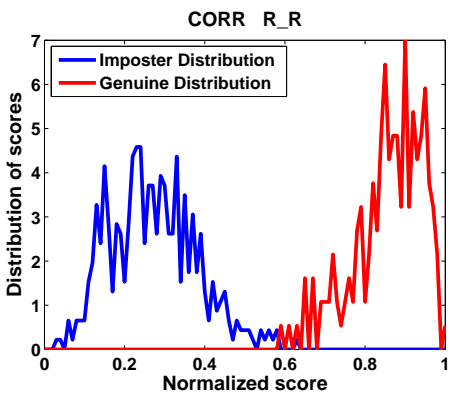

(h)

Figure C.3 Data collection 2. The ROC and the distribution of scores for the correlation technique. 
Chapter $C$ Impact of intra-class variation. The ROC and the distribution of scores. Data collection 2.

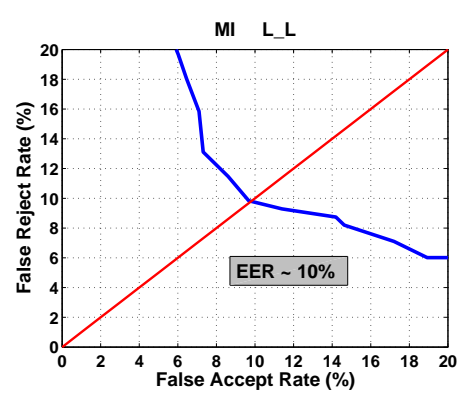

(a)

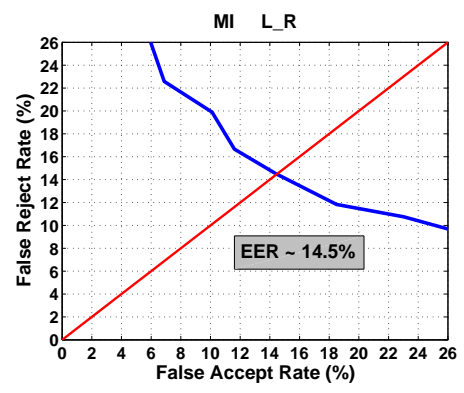

(c)

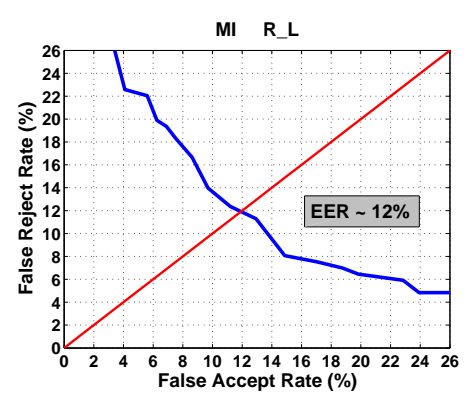

(e)

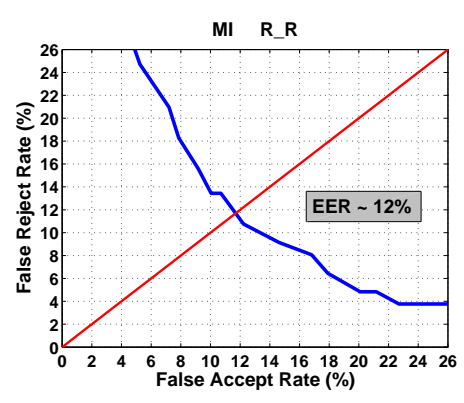

(g)

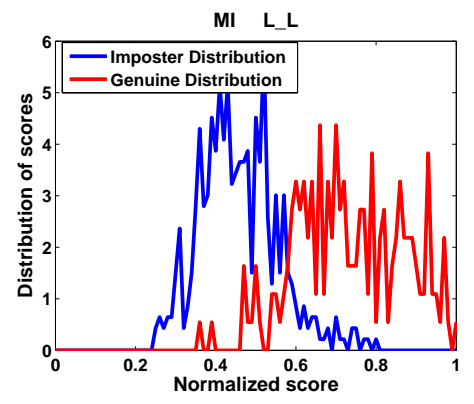

(b)

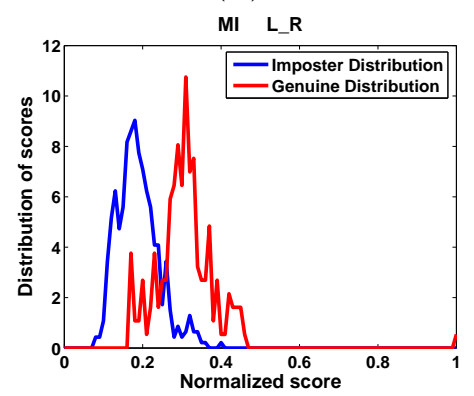

(d)

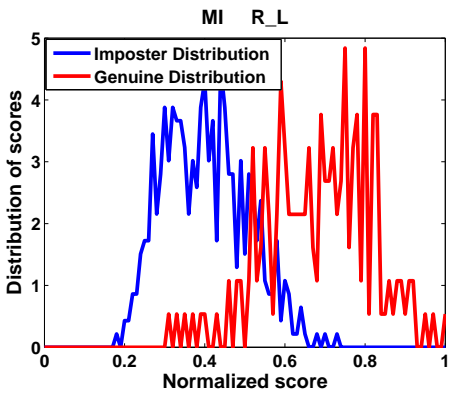

(f)

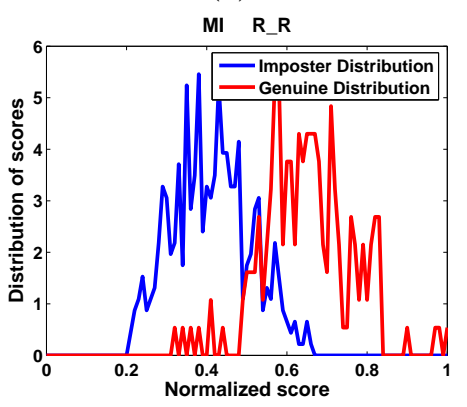

(h)

Figure C.4 Data collection 2. The ROC and the distribution of scores for the mutual information technique. 
Chapter $C$ Impact of intra-class variation. The ROC and the distribution of scores. Data collection 2.

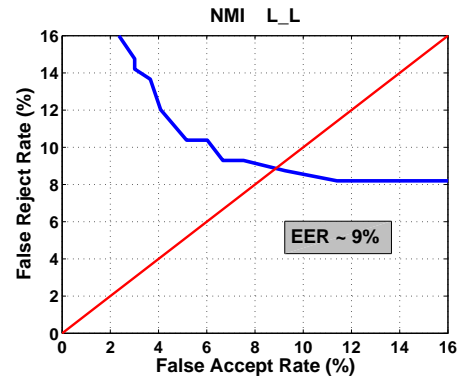

(a)

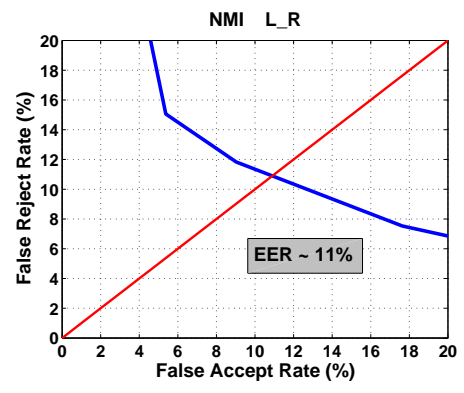

(c)

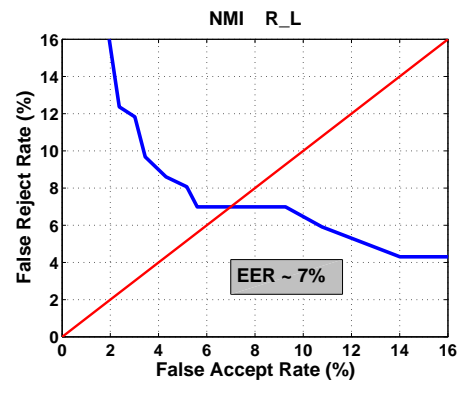

(e)

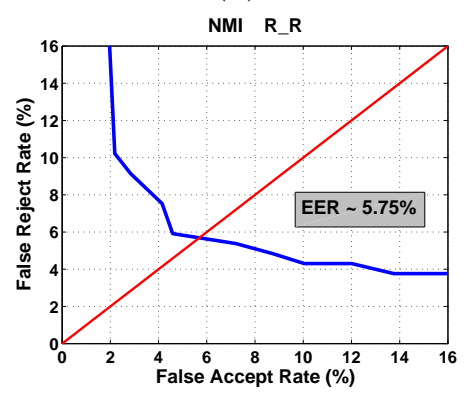

$(g)$

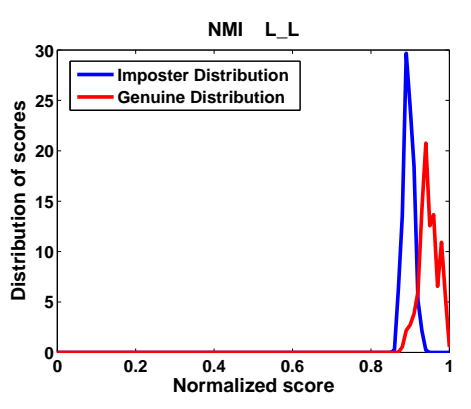

(b)

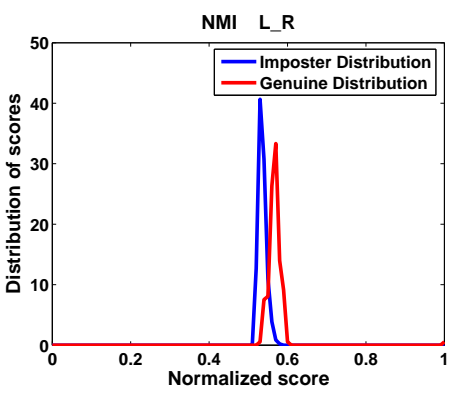

(d)

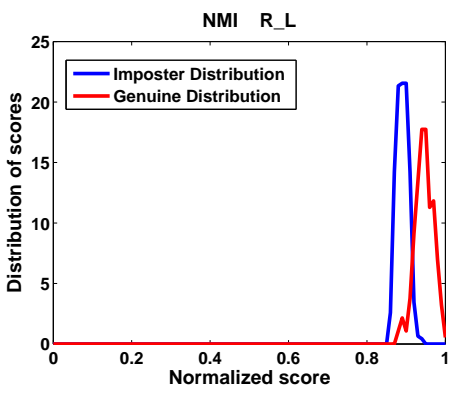

(f)

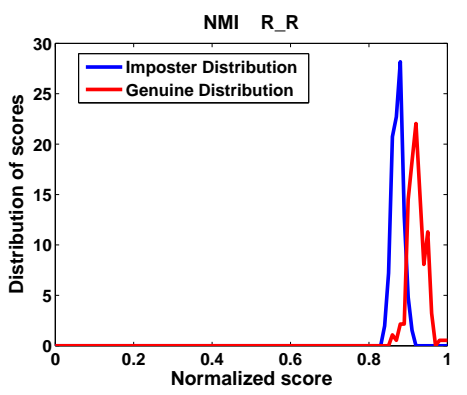

(h)

Figure C.5 Data collection 2. The ROC and the distribution of scores for the normalized mutual information technique. 
Chapter $C$ Impact of intra-class variation. The ROC and the distribution of scores. Data collection 2.

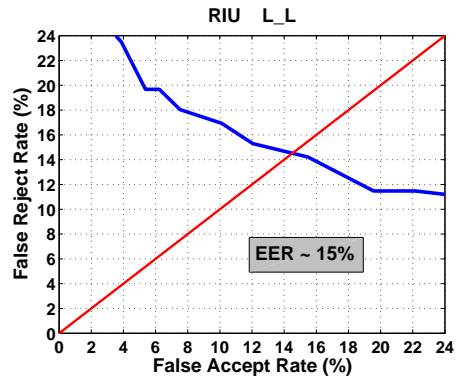

(a)

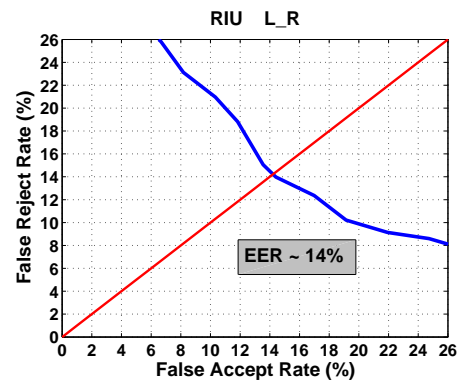

(c)

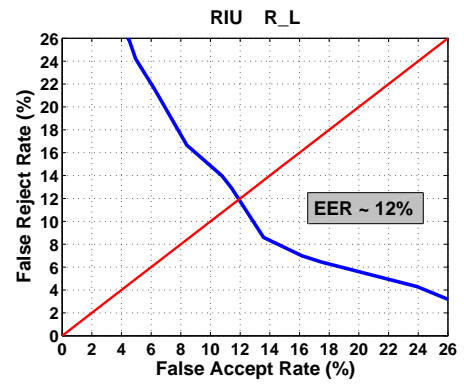

(e)

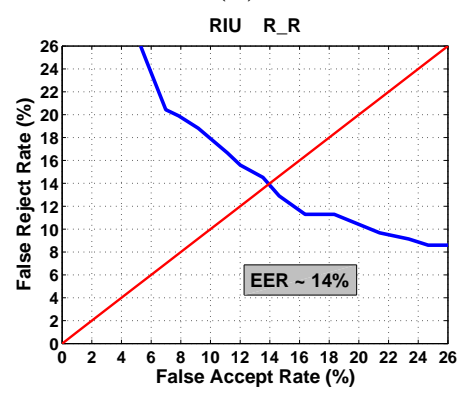

$(g)$

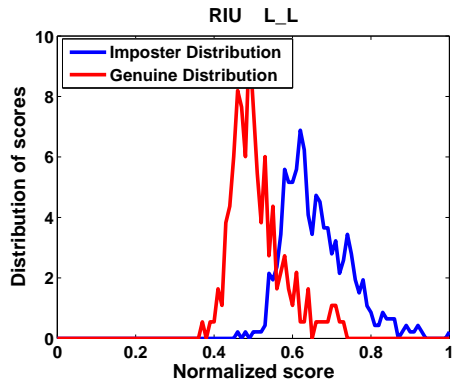

(b)

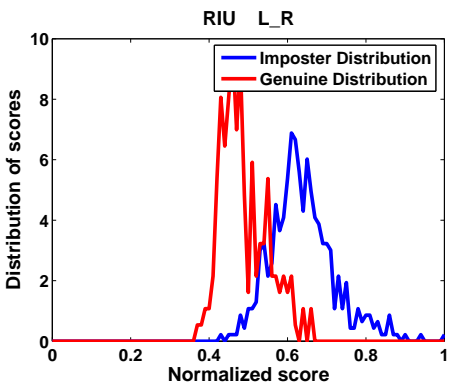

(d)

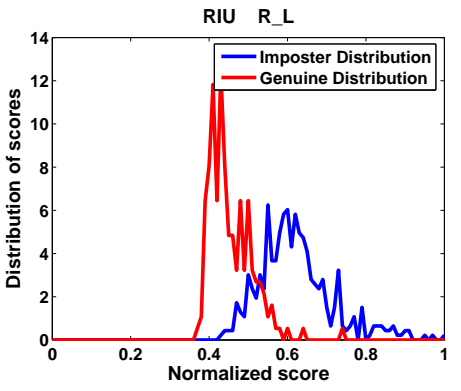

(f)

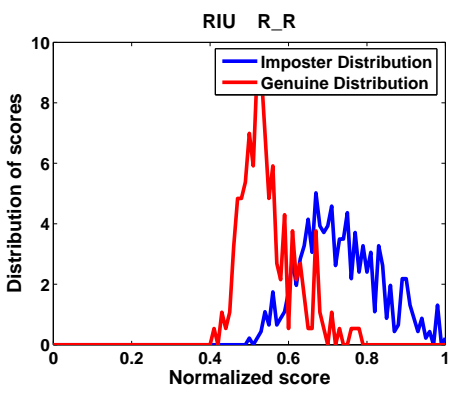

(h)

Figure C.6 Data collection 2. The ROC and the distribution of scores for the ratio-image uniformity technique. 
Chapter $C$ Impact of intra-class variation. The ROC and the distribution of scores. Data collection 2.

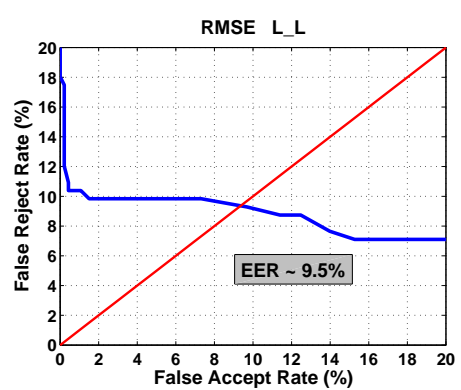

(a)

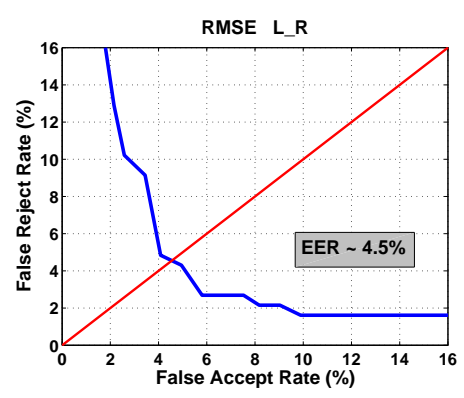

(c)

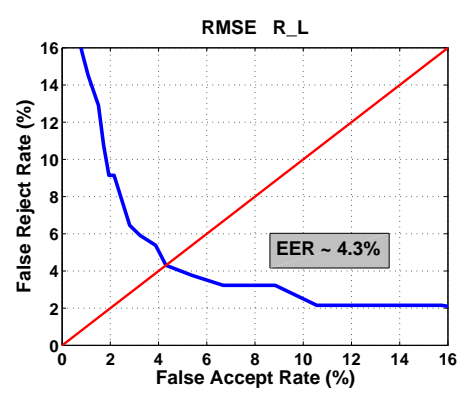

(e)

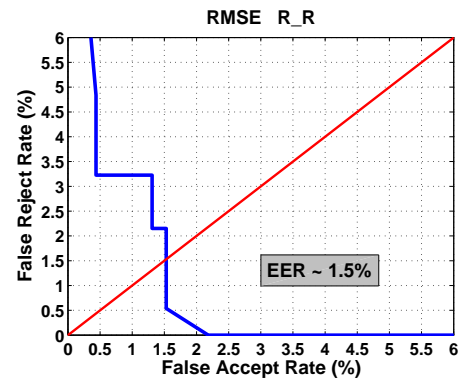

(g)

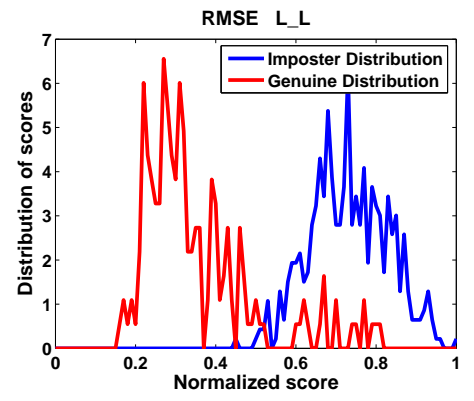

(b)

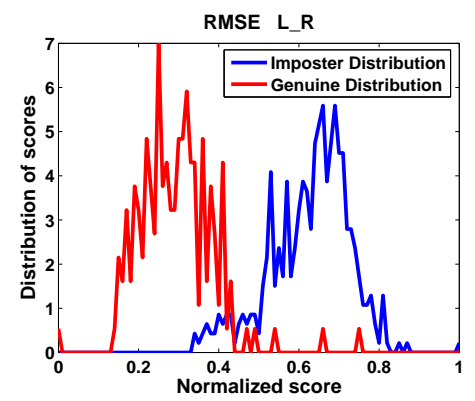

(d)

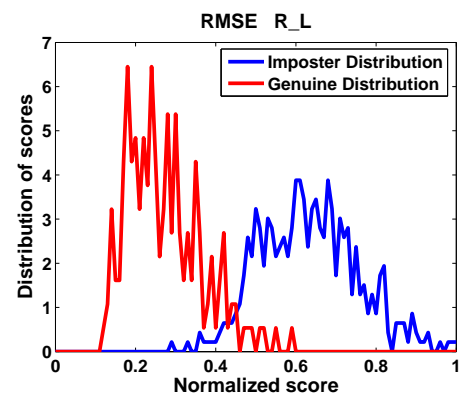

(f)

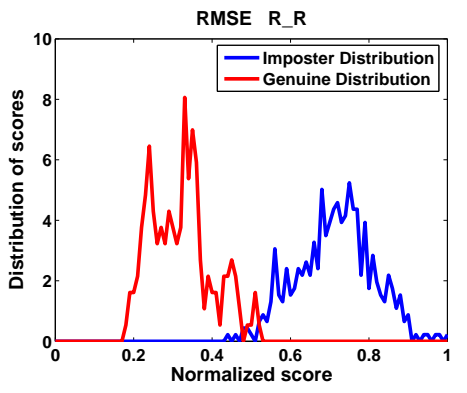

(h)

Figure C.7 Data collection 2. The ROC and the distribution of scores for the root mean square error technique. 
Chapter $C$ Impact of intra-class variation. The ROC and the distribution of scores. Data collection 2.

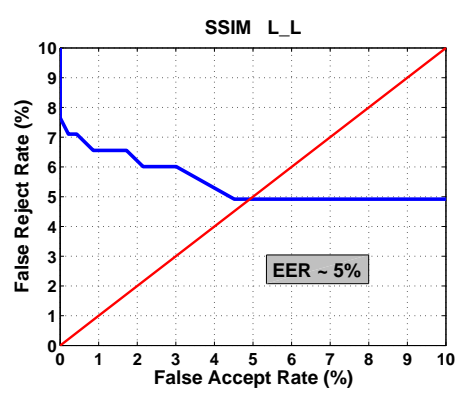

(a)

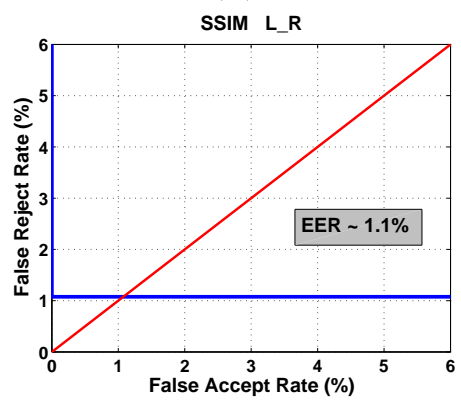

(c)

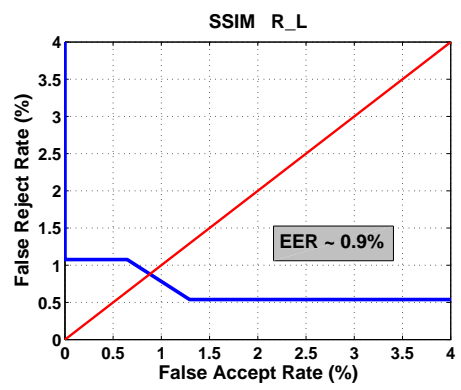

(e)

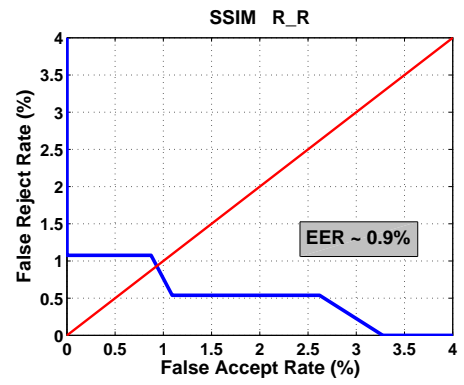

$(\mathrm{g})$

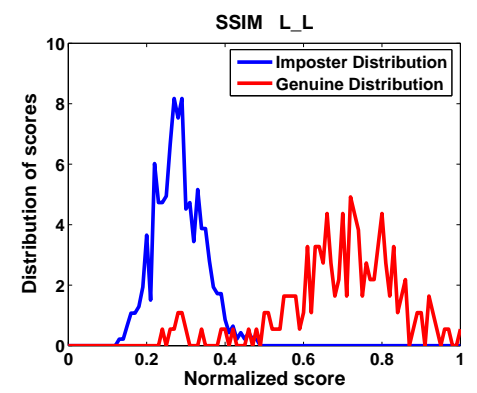

(b)

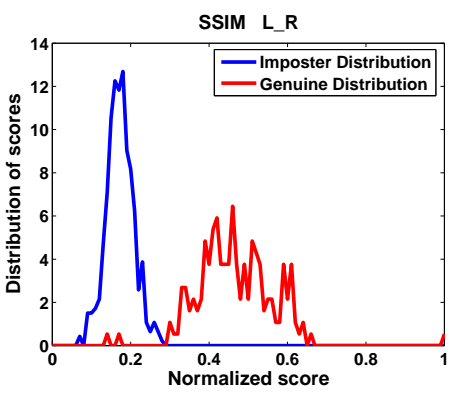

(d)

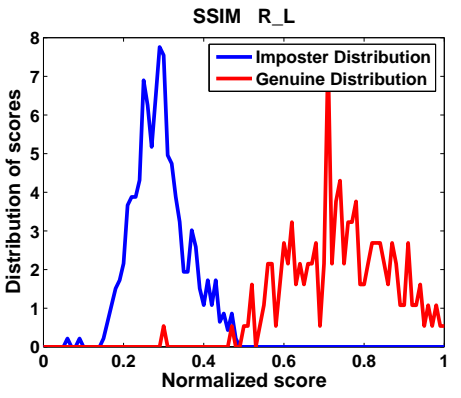

(f)

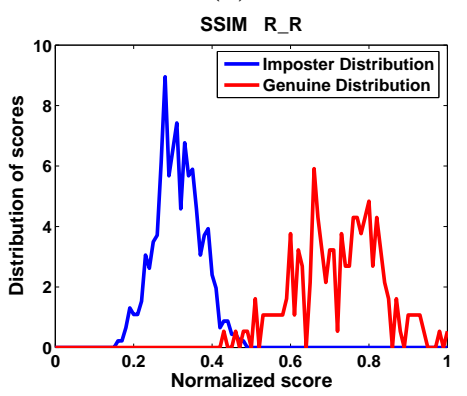

(h)

Figure C.8 Data collection 2. The ROC and the distribution of scores for the structural similarity index technique. 
Chapter $C$ Impact of intra-class variation. The ROC and the distribution of scores. Data collection 2.

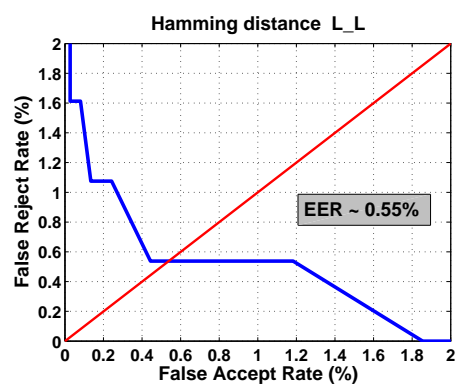

(a)

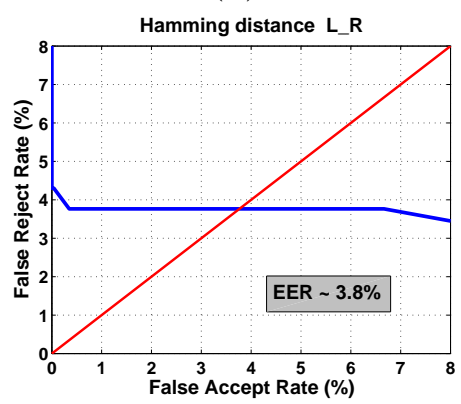

(c)

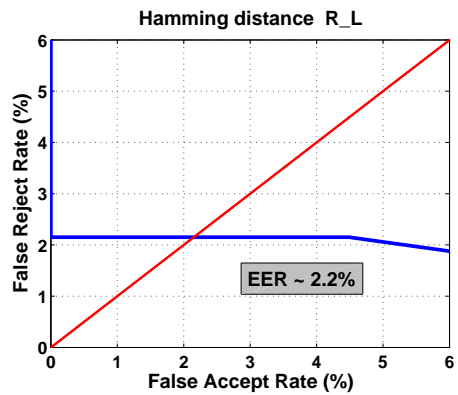

(e)

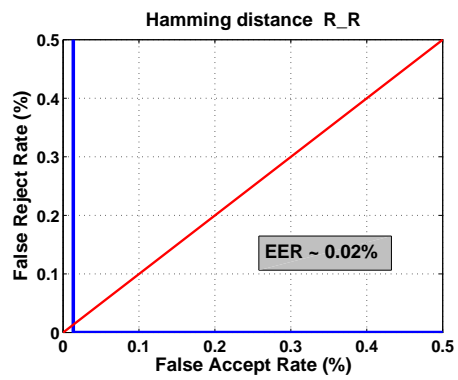

(g)

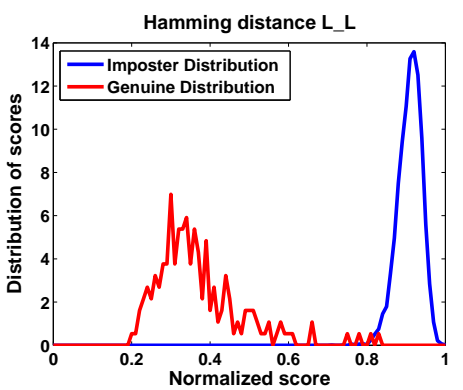

(b)

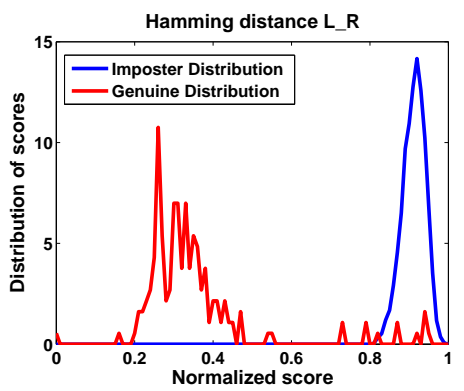

(d)

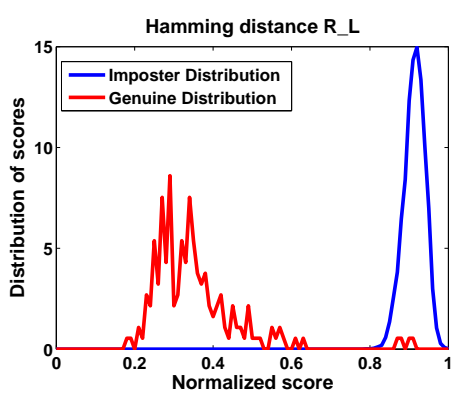

(f)

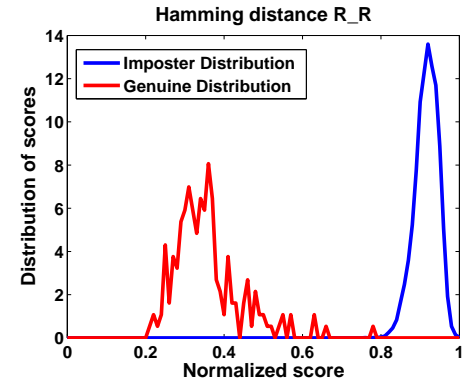

(h)

Figure C.9 Data collection 2. The ROC and the distribution of scores for the Hamming distance. 
Chapter $C$ Impact of intra-class variation. The ROC and the distribution of scores. Data collection 2.

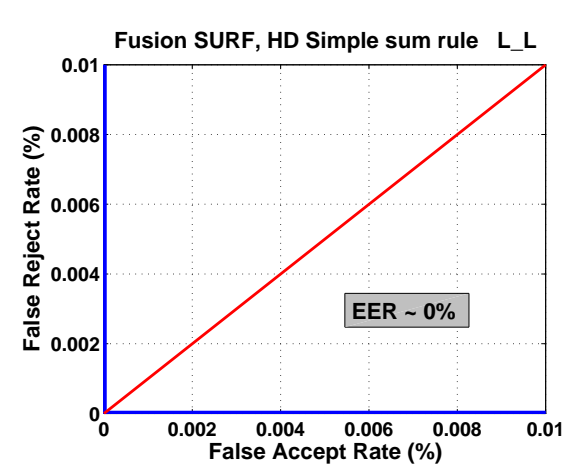

(a)

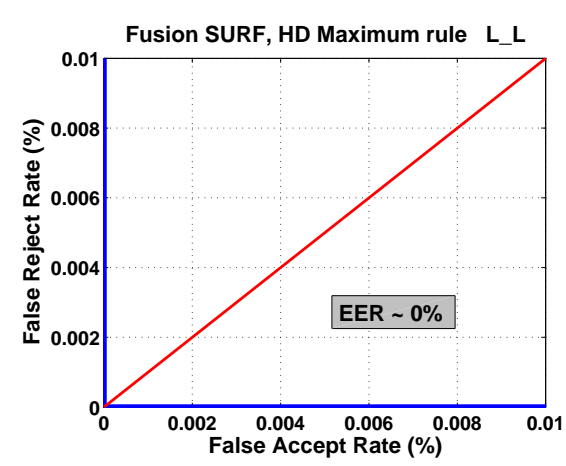

(c)

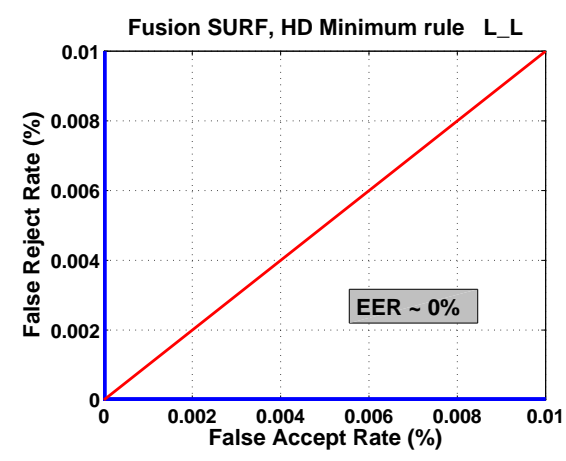

(e)

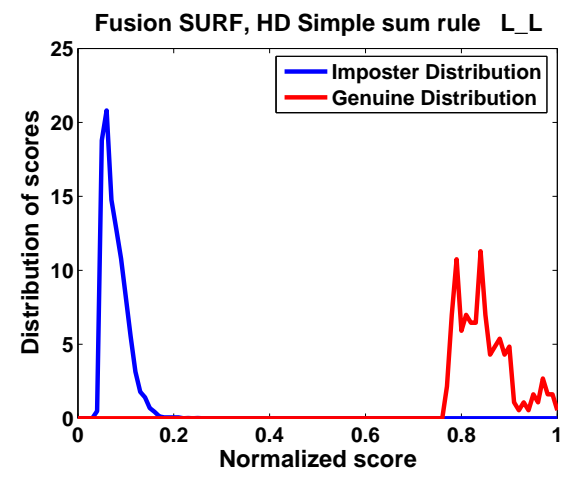

(b)

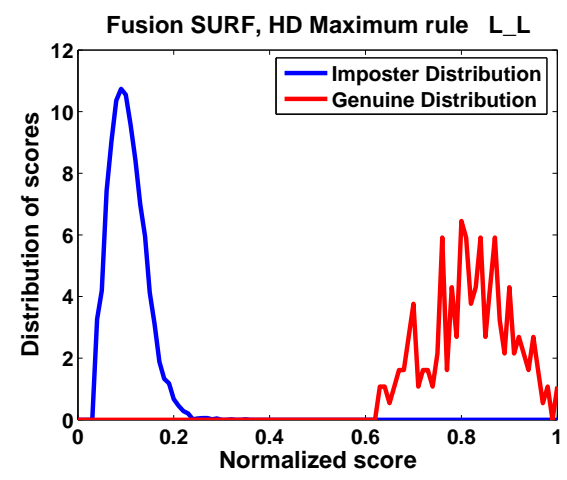

(d)

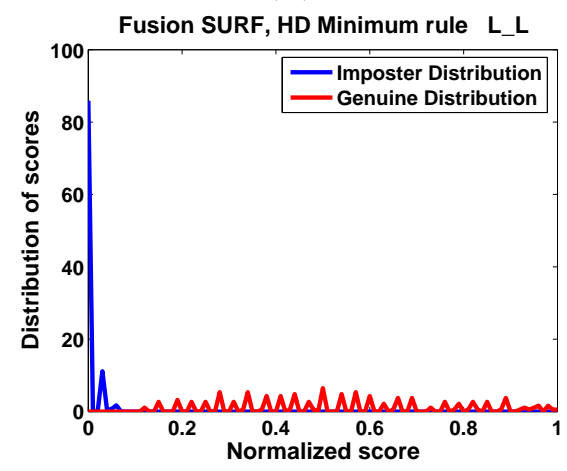

(f)

Figure C.10 Data collection 2. The fusion of iris and sclera patterns for left-eye-looking-left. The ROC and the distribution of scores. 
Chapter $C$ Impact of intra-class variation. The ROC and the distribution of scores. Data collection 2.

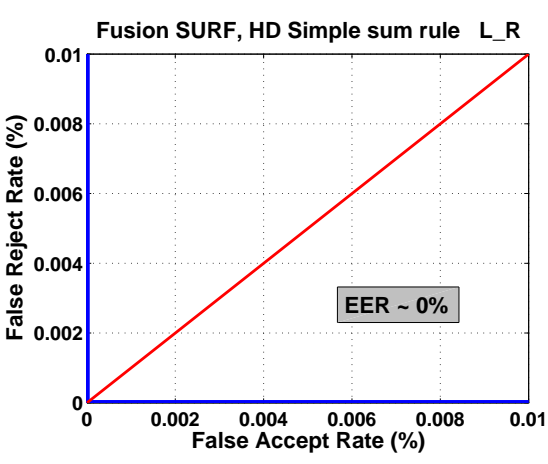

(a)

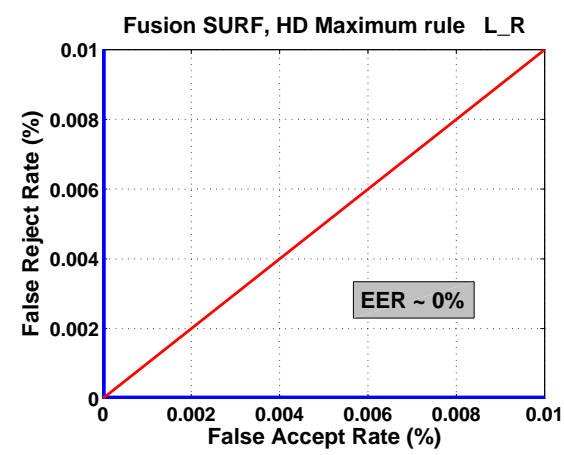

(c)

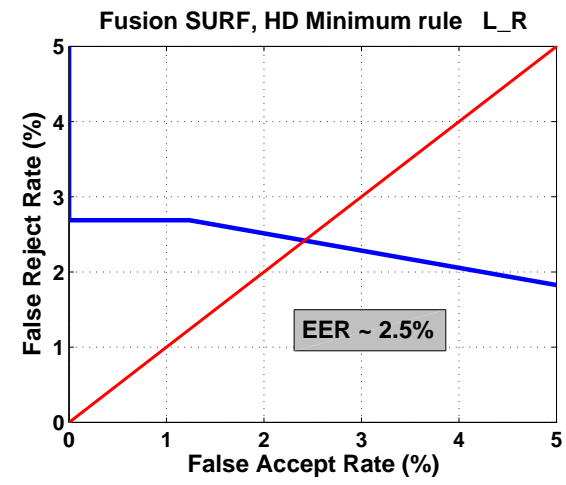

(e)

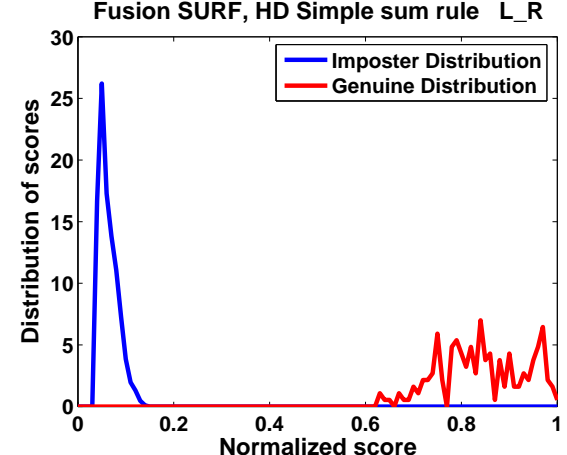

(b)

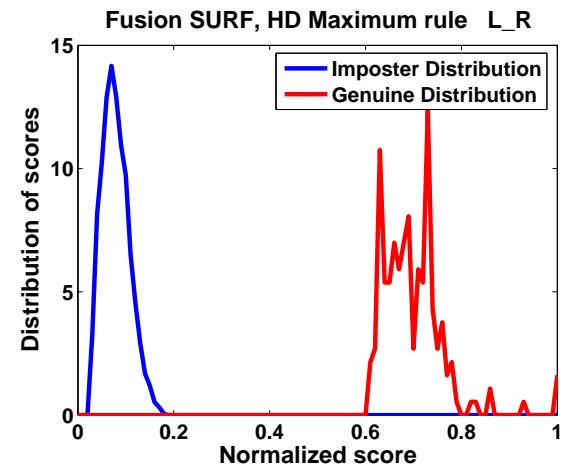

(d)

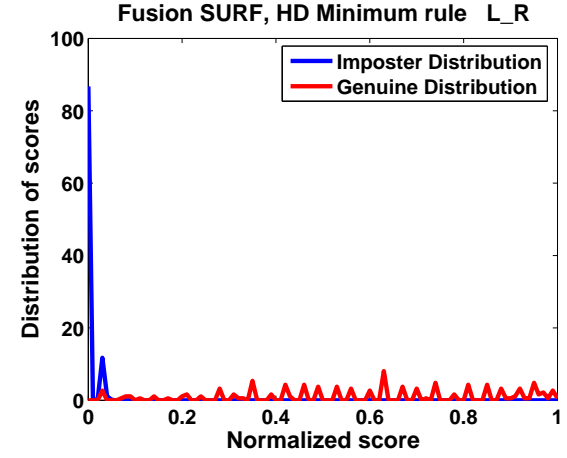

(f)

Figure C.11 Data collection 2. The fusion of iris and sclera patterns for left-eye-looking-right. The ROC and the distribution of scores. 
Chapter $C$ Impact of intra-class variation. The ROC and the distribution of scores. Data collection 2.

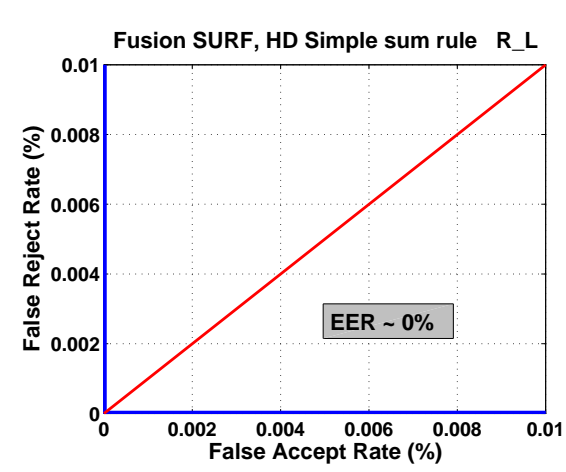

(a)

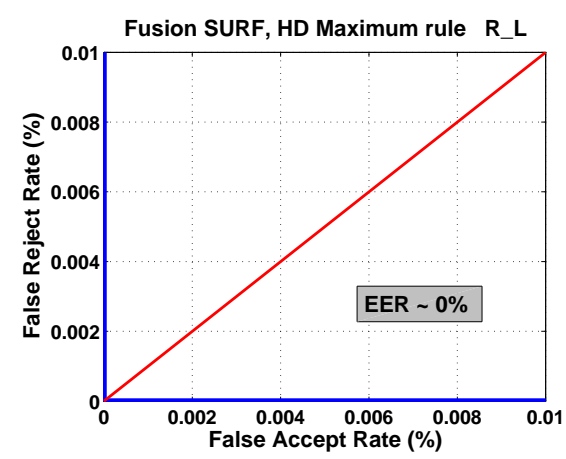

(c)

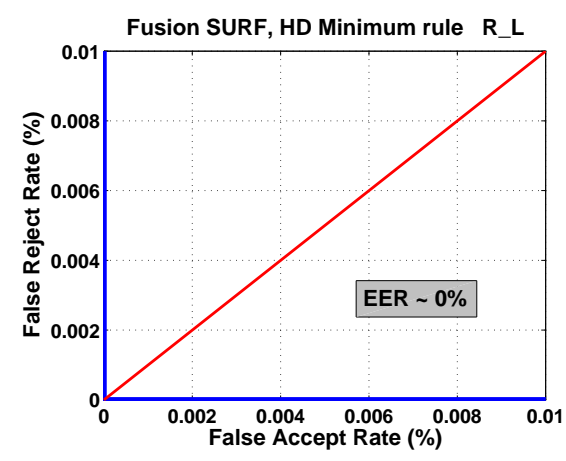

(e)

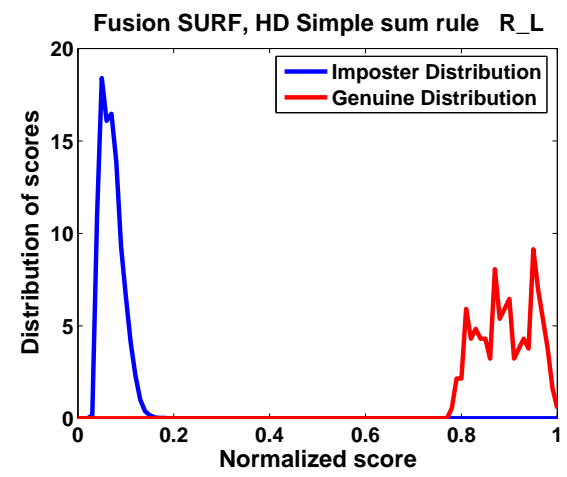

(b)

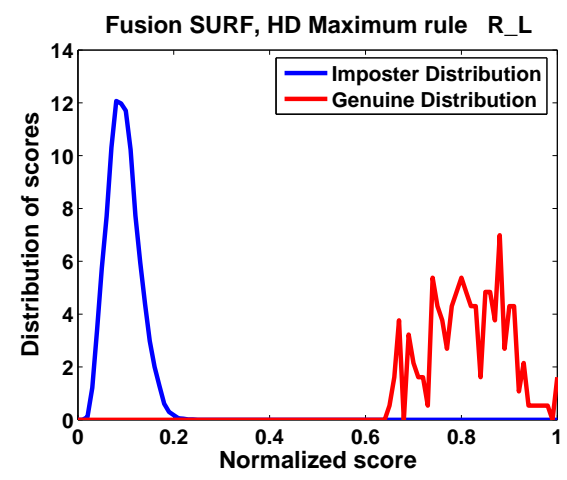

(d)

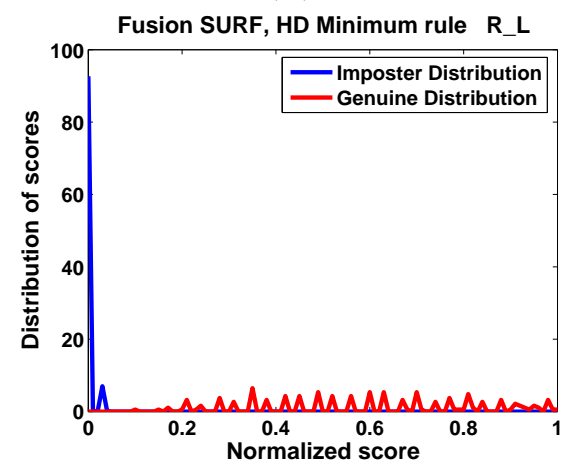

(f)

Figure C.12 Data collection 2. The fusion of iris and sclera patterns for right-eye-looking-left. The ROC and the distribution of scores. 
Chapter $C$ Impact of intra-class variation. The ROC and the distribution of scores. Data collection 2.

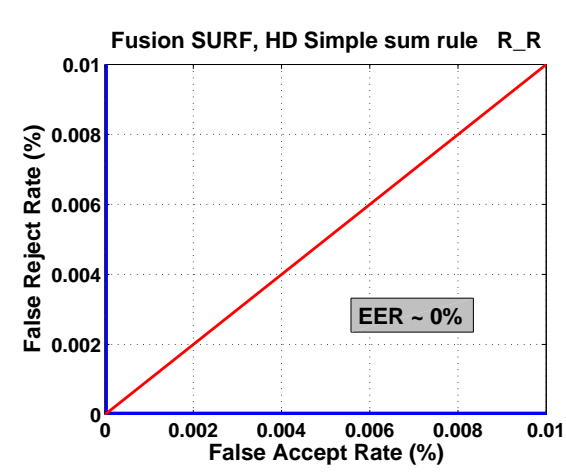

(a)

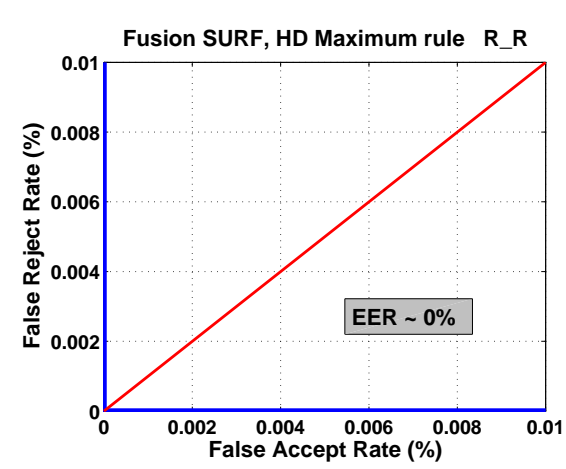

(c)

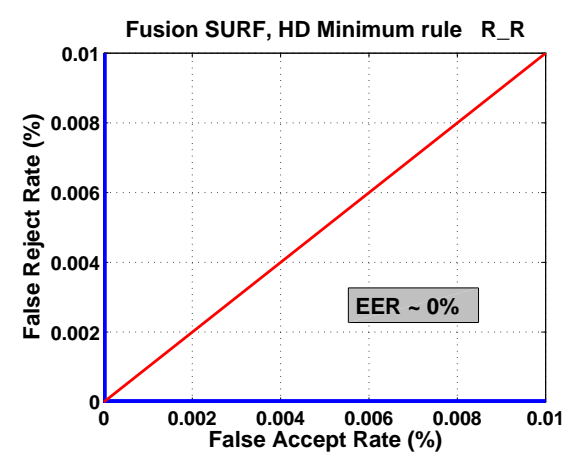

(e)

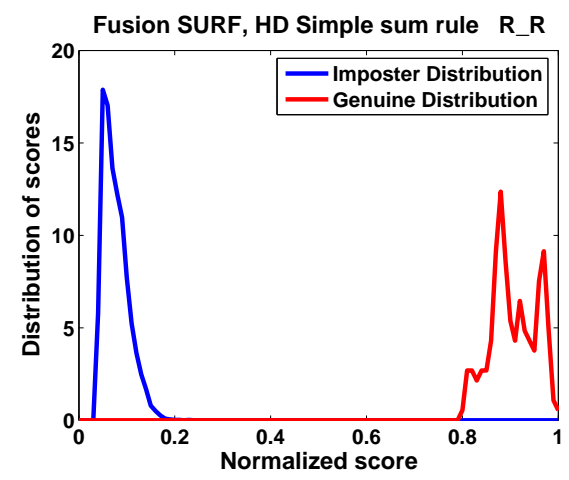

(b)

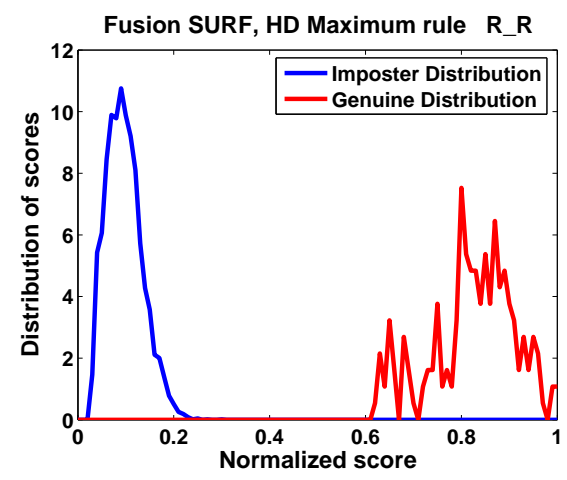

(d)

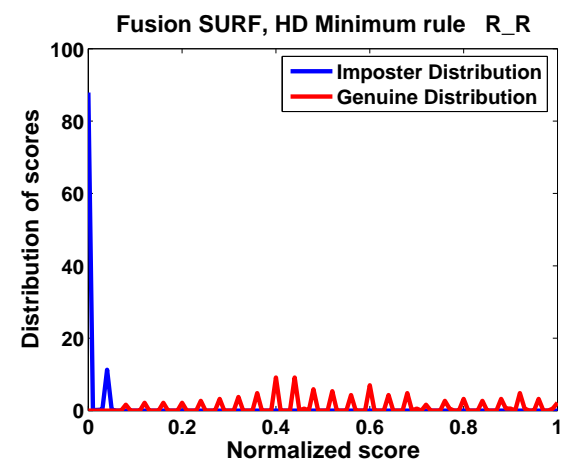

(f)

Figure C.13 Data collection 2. The fusion of iris and sclera patterns for right-eye-looking-right. The ROC and the distribution of scores. 


\section{Bibliography}

[1] I. W. Selenick, "The Double-Density Dual-Tree DWT," IEEE Transactions on Signal Processing 52, 1304-1314 (2004).

[2] A. Jain, A. Ross, and S. Prabhakar, "An Introduction to Biometric Recognition," IEEE Transactions on Circuits and Systems for Video Technology 14, $4-20(2004)$.

[3] A. Jain, R. Bolle, and S. Pankanti, in Biometrics personal Identification in Networked Society (Kluwer Academic, 2001), Chap. Retina Identification by Robert Hill, pp. 123-141.

[4] J. Daugman, "How Iris Recognition Works," IEEE Transactions on Circuit and Systems for Video Technology 14, 21-30 (2004).

[5] J. G. Daugman, "The importance of being random: statistical principles of iris recognition," Pattern Recognition 36, 279291 (2003).

[6] J. Daugman, "New Methods in Iris Recognition," IEEE Transactions on Systems, Man, and Cybernetics- Part B, Cybernetics 37, 1167-1175 (2007).

[7] A. Ross, "Iris Recognition: The Path Forward," IEEE Computer pp. 30-35 (2010). 
[8] K. W. Bowyer, K. Hollingsworth, and P. J. Flynn, "Image understanding for iris biometrics: A survey," Computer Vision and Image Understanding 110, 281-307 (2008).

[9] M. S. Hosseini, B. N. Araabi, and H. Soltanian-Zadeh, "Pigment Melanin: Pattern for Iris Recognition," IEEE Transactions on Instrumentation and Measurement 59, 792804 (2010).

[10] A. Muron and J. Pospisil, "The human iris structure and its usages," Acta Univ. Palacki. Olomuc. Fac. Rerum Nat. Phys. 39, 87-95 (2000).

[11] H. Borgen, P. Bours, and S.D.Wolthusen, "Visible-Spectrum Biometric retina Recognition," International Conference on Intelligent Information Hiding and Multimedia Signal Processing pp. 1056-1062 (2008).

[12] M. A. Amin and H. Yan, "Phase congruency based retinal vessel segmentation," Machine Learning and Cybernetics 4, 2458-2462 (2009).

[13] U. Park, R. Jillela, A. Ross, and A. K. Jain, "Periocular Biometrics in the Visible Spectrum," IEEE Transactions on Information Forensics and Security (TIFS) 6, 96-106 (2011).

[14] D. Woodard, S. Pundlik, P. Miller, R. Jillela, and A. Ross, "On the Use of Periocular and Iris Biometrics in Non-ideal Imagery," Proc. of International Conference on Pattern Recognition (ICPR) pp. 201-204 (2010), , Istanbul, Turkey.

[15] P. E. Miller, A. W. Rawls, S. J. Pundlik, and D. L. Woodard, "Personal Identification using Periocular Skin Texture," Proceedings ACM 25th Symp. applied Computing pp. 1496-1500 (2010), aCM Press. 
[16] K. Trier, in Advances in organ biology. The biology of the eye (Elsevier Inc, 2006), Vol. 10, Chap. The Sclera, pp. 353-373.

[17] J. J. Kanski, Clinical Ophthalmology: A Systematic Approach (Elsevier Science Limited, 2003).

[18] G. Heath, "The episclera, sclera and conjunctiva," Optometry today. Differential Diagnosis of Ocular Disease 9, 36-42 (2006).

[19] C. G. Owen, R. S. B. Newsom, A. R. Rudnicka, T. J. Ellis, and E. G. Woodward, "Vascular Response of the Bulbar Conjunctiva to Diabetes and Elevated Blood Pressure," Ophthalmology 112, 1801-1808 (2005).

[20] C. G. Owen, R. S. B. Newsom, A. R. Rudnicka, S. Barman, T. J. Ellis, and E. G. Woodward, "Diabetes and the Tortuosity of Vessels of the Bulbar Conjunctiva," Ophthalmology 115, e27-e32 (2008).

[21] R. C. Gonzales and R. E. Woods, Digital Image Processing, 2 ed. (Tom Robbins, Upper Saddle River, New Jersey, 07458, 2001).

[22] m. Nixon and A. Aguado, Feature Extraction and Image Processing, 2nd ed. (Elsevier Ltd., 84 Theobald's Road, London, WC1X 8RR, UK, 2008).

[23] A. K. Jain, Fundamentals of digital image processing, 1 ed. (Prentice Hall, Inc., Upper Saddle River, NJ 07458, 1989).

[24] R. Derakhshani and A. Ross, "Conjunctival Scans for Personal Identification," United States Patent, 7327860 (2008).

[25] N. D. Kalka, J. Zuo, N. A. Schmid, and B. Cukic, "Estimating and Fusing Quality Factors for Iris Biometric Images," IEEE Transactions on Systems, Man and Cybernetics, Part A: Systems and Humans 40, 509-524 (2010). 
[26] B. E. Bayer, "Color imaging array," U.S.Patent 3971065 (1976).

[27] S. Crihalmeanu, A. Ross, and R. Derakhshani, "Enhancement and Registration Schemes for Matching Conjunctival Vasculature," Proc. of International Conference on Biometrics, Alghero, Italy pp. 1240-1249 (2009).

[28] X. Li, "Demosaicing by Successive Approximation," IEEE Transactions on Image Processing 14, 370-379 (2005).

[29] I. W. Selenick, "A New Complex-Directional Wavelet Transform and its Application to Image Denoising," IEEE International Conference on Image Processing 3, 573-576 (2002).

[30] I. W. Selenick, in Wavelets in Signal And Image Analysis: from Theory to Practice (Kluwer Academic Publishers, 2001), Chap. The double density DWT, pp. 39-69.

[31] C. J. Tucker, "Red and Photographic Infrared Linear Combinations for Monitoring Vegetation," Remote Sensing Environment 8, 127-150 (1979).

[32] H. Proenca, "Iris Recognition: On the Segmentation of Degraded Images Acquired in the Visible Wavelength," IEEE Transaction on Pattern Analysis and Machine Intelligence 32, 1502-1516 (2010).

[33] L. Qiang, S. Shusuke, and D. Kunio, "Selective Enhancement Filters for Nodules, Vessels, and Airway Walls in Two or Three Dimentional CT Scans," Medical Physics 30 (2003).

[34] S. Periaswamy and H. Farid, "Elastic Registration in the Presence of Intensity Variations," IEEE Transactions on Medical Imaging 22, 865-874 (2003), http://www.cs.dartmouth.edu/farid/research/registration.html. 
[35] H. Bay, A. Ess, T. Tuyleraars, and L. V. Gool, "Speeded-Up Robust Features," Computer Vision and Image Understanding (CVIU) 110, 346-359 (2008).

[36] P. A. Viola and M. J. Jones, "Rapid object detection using a boosted cascade of simple features," Computer Vision and Pattern Recognition pp. 511-518 (2001).

[37] A. Neubeck and L. V. Gool, "Efficient non-maximum suppresion," Proceedings of the 18th International Conference in Pattern Recognition (ICPR) 3, 850-855 (2006).

[38] M. Brown and D. Lowe, "Invariant features from interest point groups," British Machine Vision Conference pp. 656-665 (2002).

[39] D. Maltoni, D. Maio, A. Jain, and S. Prabhakar, Handbook of fingerprint recognition, 1 ed. (Springer Science + Business Media, Inc., 233 Spring Street, New York, NY 10013, USA, 2003).

[40] E. M. Perez, A. D. Hughes, A. V. Stanton, S. A. Tom, A. A. Bharath, and K. H. Parker, "Segmentation of Retinal Blood Vessels Based on the Second Directional Derivative and Region Growing," International Conference on Image Processing (ICIP) 2, 173-176 (1999).

[41] Z. Wang and A. C. Bovik, "Mean Squared Error: Love It or Leave It?," IEEE Signal Processing Magazine pp. 98-117 (2009).

[42] M. Sonka and J. M. Fitzpatrick, in Handbook of Medical Imaging. Medical Image Processing and Analysis (The International Society for Optical Engineering (SPIE), 2004), Vol. 2, Chap. Image registration, pp. 487-497. 
[43] F. Maes, A. Collignon, D. Vandermeulen, G. Marchal, and P. Suetens, "Multimodality Image Registration by maximization of Mutual Information," IEEE Transactions on Medical Imaging 16, 187-198 (1997).

[44] Z. Wang, A. C. Bovik, H. R. Sheikh, and E. P. Simoncelli, "Image Quality Assessment: From Error Measurement to Structural Similarity," IEEE Transactions on Image Processing 13, 600-612 (2004).

[45] A. Ross, K. Nandakumar, and A. K. Jain, Handbook of Multibiometrics (Springer Publishers, 2006).

[46] S. Crihalmeanu and A. Ross, "Multispectral Scleral Patterns for Ocular Biometric Recognition," Pattern Recognition Letters (BIOCON) (2012), accepted / To appear in 2012.

[47] I. Guarneri, M. Guarnera, G. Messina, and V. Tomaselli, "A signature analysis based method for elliptical shape," Proc. SPIE 7537 (2010).

[48] S. Crihalmeanu and A. Ross, "On the Use of Multispectral Conjunctival Vasculature as a Soft Biometric," IEEE Workshop on Applications of Computer Vision (WACV) pp. 204-211 (2011).

[49] C. Boyce, A. Ross, M. Monaco, L. Hornak, and X. Li, "Multispectral Iris Analysis: A Preliminary Study," Proceedings of Computer Vision and Pattern Recognition Workshop on Biometrics (CVPRW) (2006).

[50] X. Li, "Modeling Intra-class Variation for Non-ideal Iris Recognition," In In Springer LNCS 3832: Int. Conf. on Biometrics, pp. 419-427 (2006).

[51] J. R. Movellan, "Tutorial on Gabor Filters," Technical Report pp. 1-23 (2002). 
[52] R. T. Tan, K. Nishino, and K. Ikeuchi, "Illumination Chromaticity Estimation using Inverse-Intensity Chromaticity Space," Computer Vision and Pattern Recognition 1, 673-680 (2003).

[53] R. T. Tan and K. Ikeuchi, "Separating Reflection Components of Textured Surfaces using a Single Image," IEEE PAMI 27, 178-193 (2005).

[54] D. Stoyanov and G. Z. Yang, "Removing Specular Reflection Components for Robotic Assisted Laparoscopic Surgery," IEEE International Conference in Image Processing 3, 632-635 (2005).

[55] J. B. Park, "Detection of Specular Highlights in Color Images using a New Color Space Transformation," International Conference on Robotics and Biomimetics (ICRB) pp. 737-741 (2004).

[56] K. Zuiderveld, in Contrast limited adaptive histogram equalization. (Academic Press Professional, San Diego, CA, USA, 1994), Chap. In P. S. Heckbert, Graphics gems IV, pp. 474-485.

[57] M. Mirmehdi, X. Xie, and J. Suri, Handbook of Texture Anlysis, 1 ed. (Imperial College Press, 57 Shelton Street, Covent Garden, London WC2H 9HE, 2008). 